WSRC-TR-94-0608

Rev. 12, November 2004

\title{
Savannah River Site Approved Site Treatment Plan, 2004 Annual Update
}

\section{Volumes I and II}

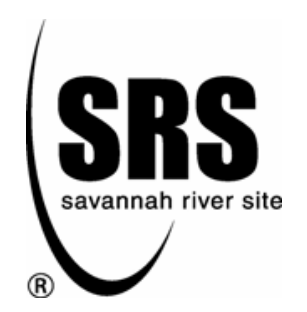

Westinghouse Savannah River Company, LLC

Savannah River Site

Aiken, SC 29808 
WSRC-TR-94-0608

Rev. 12, November 2004

\section{Savannah River Site Approved Site Treatment Plan, 2004 Annual Update (U)}

Prepared By:

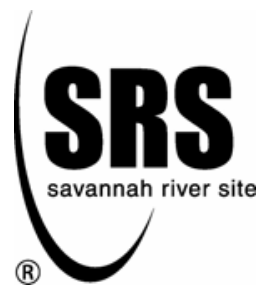

Westinghouse Savannah River Company, LLC

Savannah River Site

Aiken, SC 29808

PREPARED FOR THE U. S. DEPARTMENT OF ENERGY UNDER CONTRACT NO. DE-AC09-96SR18500 
This document was prepared in conjunction with work accomplished under Contract No. DE-AC09-96SR18500 with the U. S. Department of Energy.

\section{DISCLAIMER}

This report was prepared as an account of work sponsored by an agency of the United States Government. Neither the United States Government nor any agency thereof, nor any of their employees, makes any warranty, express or implied, or assumes any legal liability or responsibility for the accuracy, completeness, or usefulness of any information, apparatus, product or process disclosed, or represents that its use would not infringe privately owned rights. Reference herein to any specific commercial product, process or service by trade name, trademark, manufacturer, or otherwise does not necessarily constitute or imply its endorsement, recommendation, or favoring by the United States Government or any agency thereof. The views and opinions of authors expressed herein do not necessarily state or reflect those of the United States Government or any agency thereof.

This report has been reproduced directly from the best available copy.

Available for sale to the public, in paper, from: U.S. Department of Commerce, National Technical Information Service, 5285 Port Royal Road, Springfield, VA 22161, phone: (800) 553-6847, fax: (703) 605-6900

email: orders@ntis.fedworld.gov

online ordering: http://www.ntis.gov/help/index.asp

Available electronically at http://www.osti.gov/bridge

Available for a processing fee to U.S. Department of Energy and its contractors, in paper, from: U.S. Department of Energy, Office of Scientific and Technical Information, P.O. Box 62, Oak Ridge, TN 37831-0062,

phone: (865)576-8401,

fax: (865)576-5728

email: $\underline{\text { reports@ adonis.osti.gov }}$ 


\section{Table of Contents Volume I}

\section{Volume I - Compliance Plan Volume}

Chapter 1. Purpose and Scope of the Compliance Plan Volume...................................................... 1-1

Chapter 2. Key Order Provisions ……............................................................................. 2-1

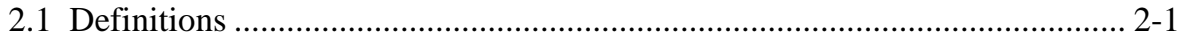

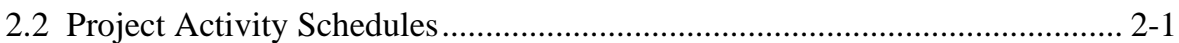

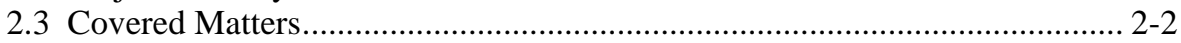

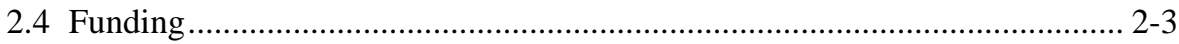

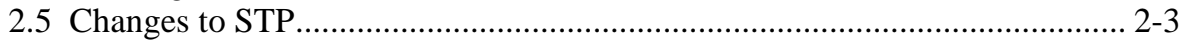

Chapter 3. Mixed Low-Level Waste Treatment........................................................................ 3-1

3.1 Mixed Low-Level Waste Streams with Treatment Capacity ............................ 3-1

3.2 Mixed Low-Level Waste Streams Requiring

Technology Development............................................................................ 3-9

3.3 Mixed Low-Level Waste Streams for

Which Further Characterization is Required .................................................. 3-10

3.4 Mixed Low-Level Waste Streams Requiring Radionuclide

Decay Prior to LDR Treatment....................................................................... 3-10

3.5 Mixed Low-Level Waste Streams - Other Commitments ................................ 3-11

Chapter 4. Mixed TRU (MTRU) Waste Streams ............................................................................. 4-1

4.1 Site MTRU Waste Management Approach ……................................................. 4-1

Chapter 5. High-Level Radioactive Waste (HLW) ……........................................................... 5-1

Appendix A. Current Fiscal Year Commitments Federal Fiscal Year 2004 .................................... A-1

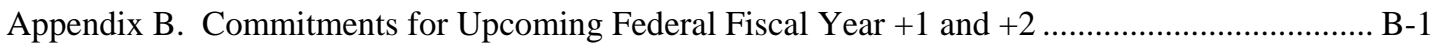

\section{List of Tables}

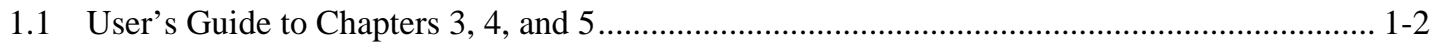

\section{List of Waste Streams}

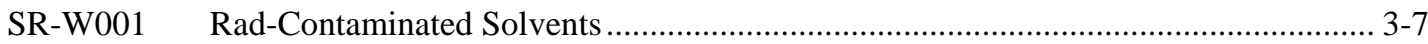

SR-W002 Rad-Contaminated Chlorofluorocarbons ............................................................... 1-2

SR-W003 Solvent Contaminated Debris (LLW) .................................................................. 3-7

SR-W004 M-Area Plating Line Sludge from Supernate Treatment ………............................... 1-2

SR-W005 Mark 15 Filtercake .................................................................................... 1-2

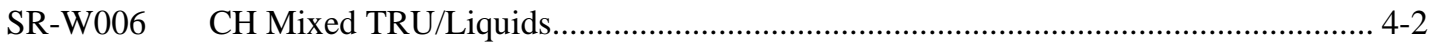

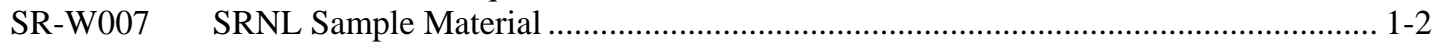

SR-W008 Separations Area Sample Receipts from SRNL ……............................................ 1-2

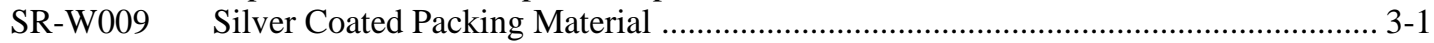

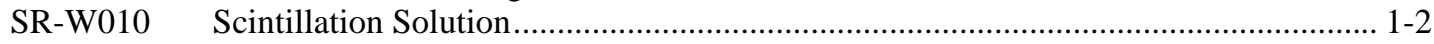

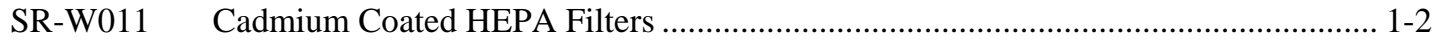

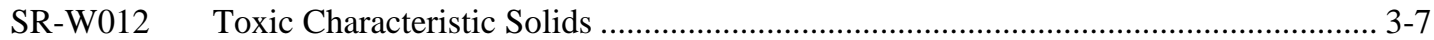

SR-W013 Low Level Waste (LLW) Lead - To be Decontaminated Onsite ............................... 1-2

SR-W014 Elemental (Liquid) Mercury - Sitewide............................................................ 3-6

SR-W015 Tritium Contaminated Equipment ....................................................................... 1-2

SR-W016 221-F Canyon High-Level Liquid Waste .................................................................... 5-1 
SR-W017

SR-W018

SR-W019

SR-W020

SR-W021

SR-W022

SR-W023

SR-W024

SR-W025

SR-W026

SR-W027

SR-W028

SR-W029

SR-W030

SR-W031

SR-W032A

SR-W032B

SR-W033

SR-W034

SR-W035

SR-W036

SR-W037

SR-W038

SR-W039

SR-W040

SR-W041

SR-W042

SR-W043

SR-W044

SR-W045

SR-W046

SR-W047

SR-W048

SR-W049

SR-W050

SR-W051

SR-W052

SR-W053

SR-W054

SR-W055

SR-W056

SR-W057

SR-W058

SR-W059

SR-W060

SR-W061

SR-W062

SR-W063

SR-W064

SR-W065

SR-W066

SR-W067

SR-W068

SR-W069

SR-W070

SR-W071
221-H Canyon High-Level Liquid Waste ................................................................... 5-1

Listed Incinerable Solids .................................................................................... 3-7

244-H RBOF High Activity Liquid Waste................................................................. 1-3

Salt Processing Filters ......................................................................................... 1-3

Poisoned Catalyst Material..................................................................................... 1-3

DWPF Benzene ....................................................................................... 1-3

Cadmium Safety/Control Rods.............................................................................. 1-3

Mercury/Tritium Gold Traps............................................................................ 1-3

Solvent/TRU Job Control Waste $<100 \mathrm{nCi} / \mathrm{g}$...................................................... 1-3

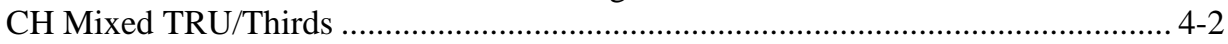

CH Mixed TRU/F-Listed Solvents...................................................................... 4-2

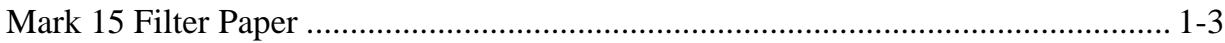

M-Area Sludge Treatability Samples ………..................................................... 1-3

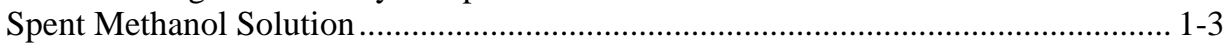

Uranium/Chromium Solution ............................................................................... 1-3

Mercury-Contaminated Heavy Water ....................................................................... 1-3

Mercury-Contaminated Heavy Water Residues .......................................................... 1-3

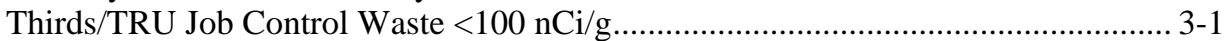

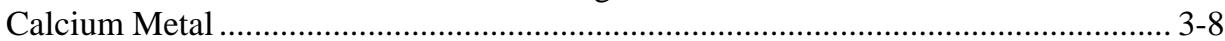

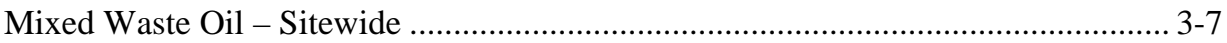

Tritiated Oil with Mercury ................................................................................... 3-10

M-Area Plating Line Sludges ......................................................................... 1-4

Plating Line Sump Material .............................................................................. 1-4

Nickel Plating Line Solution .............................................................................. 1-4

Listed Stabilized Sludge/Listed LDR-Compliant Debris ........................................... 1-4

Aqueous Mercury and Lead .............................................................................. 3-1

Paints and Thinners . ...................................................................................... 3-7

Lab Waste with Tetraphenyl Borate ....................................................................... 1-4

Tri-Butyl-Phosphate \& n-Paraffin-TRU ........................................................... 1-4

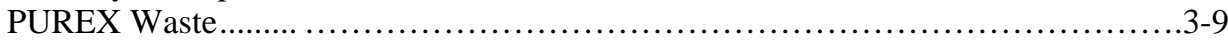

Consolidated Incineration Facility Ash ............................................................. 1-4

Consolidated Incineration Facility Blowdown ........................................................ 1-4

Soils from Spill Remediation ............................................................................. 3-7

Tank E-3-1 Clean Out Material.......................................................................... 1-4

Waste to Support High-Level Waste (HLW) Processing Demonstrations.................. 1-4

Spent Filter Cartridges and Carbon Filter Media ………........................................... 3-8

Cadmium Contaminated Glovebox Section ........................................................ 1-5

Rocky Flats Ash ............................................................................................... 4-2

Enriched Uranium Contaminated with Lead ......................................................... 1-5

Job Control Waste Containing Solvent Contaminated Wipes................................... 3-8

Job Control Waste with Enriched Uranium and Solvent Contaminated Wipes ........... 3-9

D-Tested Neutron Generators ……………………............................................. 1-5

Mixed Sludge Waste with Mercury from DWPF Treatability Studies........................ 1-5

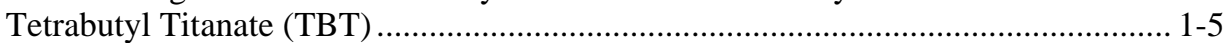

Tritiated Water with Mercury............................................................................. 3-1

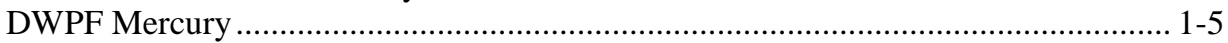

Low-Level Contaminated Debris ……………….................................................... 3-4

Macroencapsulated Low-Level Waste (TC) ……….............................................. 1-5

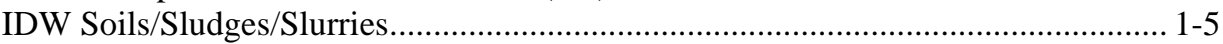

IDW Monitoring Well Purge/Development Water .................................................... 1-5

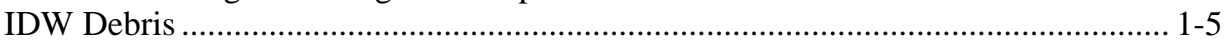

IDW Personal Protective Equipment (PPE) Waste ................................................. 1-6

Elemental (Liquid) Mercury-Sitewide ..................................................................... 3-6

Low-Level Waste (LLW) Lead - To be Macroencapsulated Offsite........................... 3-5

Mixed Waste from Laboratory Samples.......................................................................... 3-8

Wastewater Suitable for Thermal Treatment or Stabilization ................................... 3-8 
SR-W072 Supernate or Sludge Contaminated Debris from High-Level

Waste (HLW) Operations .............................................................................. 1-6

SR-W073 Cadmium-Containing Raschig Rings ...................................................................... 3-7

SR-W077 Aqueous Characteristic Wastewater............................................................................ 3-1

SR-W078 LDR Hazardous Waste Awaiting Radiological Screening ........................................... 3-10

SR-W079 Polychlorinated Biphenyl (PCB) Mixed Waste …………….................................... 3-3

SR-W080 Charleston Naval Shipyard Waste.............................................................................. 3-8

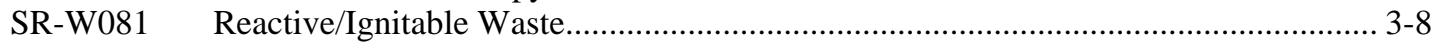

SR-W082 Radioactive Chemicals, Metals, and Pesticides (CMP) Soil ....................................... 3-7

SR-W083 Mercury- and Chromium-Contaminated Residues.................................................... 1-7

SR-W084 Remediation Waste Soils that Meet LDR …........................................................ 1-7

SR-W085 Rocky Flats Plutonium Fluoride Residues ................................................................. 1-7

SR-W086 Characteristically Hazardous Non-Incinerable Solids ….......................................... 3-6

SR-W087 Bettis Atomic Power Laboratory Contaminated Oil ................................................ 3-8

SR-W088 Sludges and Non-Debris Solids ...................................................................... 3-7

SR-W089 TRU Mixed Waste from Mound Site ...................................................................... 4-2

SR-W090 Elemental Mercury - High Rad ……......................................................................

SR-W091 Contaminated Debris (High Rad)............................................. 


\section{Chapter 1. Purpose and Scope of the Compliance Plan Volume}

For each facility at which the United States (U.S.) Department of Energy (DOE) generates or stores mixed wastes, Section 3021(b) of the Resource Conservation and Recovery Act (RCRA), 42 U.S.C. 6721, as added by Section 105(a) of the Federal Facility Compliance Act (P. L. 102-386, the FFCAct), requires DOE to devise a plan for developing treatment capacities and technologies to treat mixed waste. Upon submission of a plan to the South Carolina Department of Health and Environmental Control (SCDHEC), the FFCAct requires SCDHEC to solicit and consider public comments, and approve, approve with modification, or disapprove the plan, within six months. The agency is to consult with U.S. Environmental Protection Agency (EPA) and any state in which a facility affected by the plan is located. Upon approval of a plan, SCDHEC shall issue an order requiring compliance with the approved plan (Order).

The U.S. Department of Energy, Savannah River Operations Office (DOE-SR), has prepared the Site Treatment Plan (STP) for Savannah River Site (SRS) mixed wastes in accordance with RCRA Section 3021(b), and SCDHEC has approved the STP (except for certain offsite wastes) and issued an order enforcing the STP commitments in Volume I. DOE-SR and SCDHEC agree that this STP fulfills the requirements contained in the FFCAct, RCRA Section 3021, and, therefore, pursuant to Section 105(a) of the FFCAct (RCRA Section 3021(b)(5)), DOE's requirements are to implement the plan for the development of treatment capacities and technologies pursuant to RCRA Section 3021.

Emerging and new technologies not yet considered may be identified to manage waste more safely, effectively, and at lower cost than technologies currently identified in the plan. DOE will continue to evaluate and develop technologies that offer potential advantages in public acceptance, privatization, consolidation, risk abatement, performance, and life-cycle cost. Should technologies that offer such advantages be identified, DOE may request a revision/modification of the STP in accordance with the provisions of Consent Order 95-22-HW.

The Compliance Plan Volume (Volume I) identifies project activity schedule milestones for achieving compliance with Land Disposal Restrictions (LDR). Information regarding the technical evaluation of treatment options for SRS mixed wastes is contained in the Background Volume (Volume II) and is provided for information.

Changes to the STP will be made in accordance with the provisions of Consent Order 95-22-HW.

Table 1.1 is a user's guide that is provided as an aid in reviewing waste stream information in the STP. The table provides the status of each waste stream, the preferred treatment option (PO), and the primary location(s) of the waste streams in Volume I and II of the STP. Waste streams that have been eliminated, combined, are in compliance, or will be in compliance by November 15, 2003, do not appear in Volume I other than this table.

In 1995, DOE Headquarters expanded the scope of the master complex-wide database used to maintain mixed waste inventory data and to generate the 1995 Mixed Waste Inventory Report (MWIR). Non-mixed TRU data was incorporated into the database, which was re-named as the Material Inventory and Tracking Information (MITI) database. With the expansion of the database, certain non-mixed TRU waste streams were assigned waste stream numbers SR-W074 through SR-W076. Maintenance of the database on a complex-wide basis was discontinued after fiscal year 1996. Waste stream numbers for future SRS mixed waste streams are expected to remain sequential. 
Table 1.1 User's Guide to Chapters 3, 4, and 5

\begin{tabular}{|c|c|c|c|c|c|}
\hline $\begin{array}{c}\text { Waste Stream } \\
\text { No. }\end{array}$ & $\begin{array}{l}\text { Waste Stream } \\
\text { Name }\end{array}$ & $\begin{array}{l}\text { Preferred Option } \\
\text { (PO) }\end{array}$ & $\begin{array}{l}\text { Volume I Section } \\
\text { Identification }\end{array}$ & $\begin{array}{c}\text { Volume II } \\
\text { Section } \\
\text { Identification } \\
\end{array}$ & Waste Stream Status \\
\hline SR-W001 & $\begin{array}{l}\text { Rad-Contaminated } \\
\text { Solvents }\end{array}$ & $\begin{array}{c}\text { Combustion at } \\
\text { Commercial Facility }\end{array}$ & 3.1 .2 .6 & 3.1 .2 .6 & Ongoing Treatment \\
\hline SR-W002 & $\begin{array}{l}\text { Rad-Contaminated } \\
\text { Chlorofluorocarbons }\end{array}$ & $\begin{array}{l}\text { Consolidated with } \\
\text { SR-W001 }\end{array}$ & N/A & 1.4, Table 1.1 & $\begin{array}{l}\text { Consolidated with SR- } \\
\text { W001 }\end{array}$ \\
\hline SR-W003 & $\begin{array}{c}\text { Solvent } \\
\text { Contaminated Debris } \\
\text { (LLW) }\end{array}$ & $\begin{array}{c}\text { Thermal Treatment, } \\
\text { Macroencapsulation } \\
\text { or Stabilization and/or } \\
\text { alternative debris } \\
\text { treatment at } \\
\text { Commercial Vendor } \\
\text { Facility } \\
\end{array}$ & 3.1 .2 .6 & 3.1 .2 .6 & Ongoing Treatment \\
\hline SR-W004 & $\begin{array}{l}\text { M-Area Plating Line } \\
\text { Sludge from } \\
\text { Supernate Treatment }\end{array}$ & $\begin{array}{l}\text { Consolidated with } \\
\text { SR-W037 }\end{array}$ & N/A & 1.4 , Table 1.1 & $\begin{array}{c}\text { Consolidated with SR- } \\
\text { W037 }\end{array}$ \\
\hline SR-W005 & Mark 15 Filtercake & $\begin{array}{l}\text { Stabilization by } \\
\text { Vitrification- } \\
\text { M-Area Vendor } \\
\text { Treatment Facility }\end{array}$ & N/A & 3.5 .3 & Waste Stream Treated \\
\hline SR-W006 & $\begin{array}{c}\text { CH Mixed } \\
\text { TRU/Liquids }\end{array}$ & $\begin{array}{l}\text { Characterization at } \\
\text { SRS-WIPP Disposal }\end{array}$ & 4.1 & 4.2 .2 & $\begin{array}{c}\text { Awaiting } \\
\text { Characterization }\end{array}$ \\
\hline SR-W007 & $\begin{array}{c}\text { SRNL Sample } \\
\text { Material* }\end{array}$ & $\begin{array}{l}\text { Waste Stream } \\
\text { Eliminated* }\end{array}$ & N/A & $\begin{array}{l}\text { Appendix to } \\
\text { Chapter } 3 \\
\end{array}$ & $\begin{array}{l}\text { Waste Stream } \\
\text { Eliminated* }\end{array}$ \\
\hline SR-W008 & $\begin{array}{l}\text { Separations Area } \\
\text { Sample Receipts } \\
\text { from SRNL* }\end{array}$ & $\begin{array}{c}\text { Vitrification, } \\
\text { Stabilization, or WW } \\
\text { Treatment* }^{*}\end{array}$ & N/A & $\begin{array}{l}\text { Appendix to } \\
\text { Chapter } 3\end{array}$ & Ongoing Treatment* \\
\hline SR-W009 & $\begin{array}{c}\text { Silver Coated } \\
\text { Packing Material }\end{array}$ & $\begin{array}{c}\text { Macroencapsulation } \\
\text { in a Steel Container- } \\
\text { Onsite via a } \\
\text { Treatability Variance }\end{array}$ & 3.1 .1 .3 & 3.1 .1 .3 & $\begin{array}{c}\text { Awaiting Variance } \\
\text { Approval }\end{array}$ \\
\hline SR-W010 & Scintillation Solution & $\begin{array}{l}\text { Consolidated with } \\
\text { SR-W001 }\end{array}$ & N/A & 1.4 , Table 1.1 & $\begin{array}{c}\text { Consolidated with SR- } \\
\text { W001 }\end{array}$ \\
\hline SR-W011 & $\begin{array}{l}\text { Cadmium Coated } \\
\text { HEPA Filters }\end{array}$ & $\begin{array}{l}\text { Scrap Metal } \\
\text { Exclusion }\end{array}$ & N/A & $\begin{array}{l}\text { Appendix to } \\
\text { Chapter } 3\end{array}$ & $\begin{array}{l}\text { Recycled under Scrap } \\
\text { Metal Exclusion }\end{array}$ \\
\hline SR-W012 & $\begin{array}{c}\text { Toxic Characteristic } \\
\text { Solids }\end{array}$ & $\begin{array}{c}\text { Thermal Treatment, } \\
\text { Macroencapsulation } \\
\text { or Stabilization and/or } \\
\text { alternative debris } \\
\text { treatment at } \\
\text { Commercial Facility }\end{array}$ & 3.1 .2 .6 & 3.1 .2 .6 & Ongoing Treatment \\
\hline SR-W013 & $\begin{array}{l}\text { Low-Level Waste } \\
\text { (LLW) Lead - To be } \\
\text { Decontaminated } \\
\text { Onsite }\end{array}$ & $\begin{array}{l}\text { Reclassified as } \\
\text { material in use }\end{array}$ & N/A & 3.5 .4 & $\begin{array}{l}\text { Waste Stream } \\
\text { Eliminated }\end{array}$ \\
\hline SR-W014 & $\begin{array}{c}\text { Elemental (Liquid) } \\
\text { Mercury - Sitewide* }\end{array}$ & $\begin{array}{c}\text { Amalgamation- } \\
\text { Offsite Commercial } \\
\text { Vendor }\end{array}$ & 3.1 .2 .4 & 3.1 .2 .4 & Awaiting Treatment \\
\hline SR-W015 & $\begin{array}{c}\text { Tritium } \\
\text { Contaminated } \\
\text { Equipment }\end{array}$ & $\begin{array}{l}\text { Macroencapsulation } \\
\text { in S. S. Container as } \\
\text { 90-Day Generator }\end{array}$ & N/A & $\begin{array}{l}\text { Appendix to } \\
\text { Chapter } 3\end{array}$ & Ongoing Treatment \\
\hline SR-W016 & $\begin{array}{l}\text { 221-F Canyon High- } \\
\text { Level Liquid Waste }\end{array}$ & Vitrification at DWPF & 5 & 5 & Ongoing Treatment \\
\hline
\end{tabular}


Table 1.1 User's Guide to Chapters 3, 4, and 5 (cont'd)

\begin{tabular}{|c|c|c|c|c|c|}
\hline $\begin{array}{c}\text { Waste Stream } \\
\text { No. }\end{array}$ & $\begin{array}{c}\text { Waste Stream } \\
\text { Name }\end{array}$ & $\begin{array}{l}\text { Preferred Option } \\
\text { (PO) }\end{array}$ & $\begin{array}{l}\text { Volume I Section } \\
\text { Identification }\end{array}$ & $\begin{array}{c}\text { Volume II } \\
\text { Section } \\
\text { Identification } \\
\end{array}$ & $\begin{array}{l}\text { Waste Stream } \\
\text { Status }\end{array}$ \\
\hline SR-W017 & $\begin{array}{l}\text { 221-H Canyon High- } \\
\text { Level Liquid Waste }\end{array}$ & Vitrification at DWPF & 5 & 5 & $\begin{array}{l}\text { Ongoing } \\
\text { Treatment }\end{array}$ \\
\hline SR-W018 & $\begin{array}{l}\text { Listed Incinerable } \\
\text { Solids }\end{array}$ & $\begin{array}{c}\text { Combustion at } \\
\text { Commercial Facility or } \\
\text { Macroencapsulation or } \\
\text { Stabilization at } \\
\text { Commercial Facility }\end{array}$ & 3.1 .2 .6 & 3.1 .2 .6 & $\begin{array}{l}\text { Wastestream } \\
\text { Treated }\end{array}$ \\
\hline SR-W019 & $\begin{array}{c}\text { 244-H RBOF High } \\
\text { Activity Liquid Waste }\end{array}$ & $\begin{array}{l}\text { Consolidated with SR- } \\
\text { W017* }\end{array}$ & N/A & 1.4, Table 1.1 & $\begin{array}{c}\text { Consolidated } \\
\text { with SR-W017 }\end{array}$ \\
\hline SR-W020 & $\begin{array}{l}\text { Salt Processing } \\
\text { Filters }\end{array}$ & $\begin{array}{c}\text { Future Generation - } \\
\text { Treatment to be } \\
\text { Determined }\end{array}$ & N/A & $\begin{array}{r}\text { Appendix to } \\
\text { Chapter } 3\end{array}$ & $\begin{array}{l}\text { Future } \\
\text { Generation }\end{array}$ \\
\hline SR-W021 & $\begin{array}{c}\text { Poisoned Catalyst } \\
\text { Material }\end{array}$ & $\begin{array}{r}\text { Waste Stream } \\
\text { Eliminated } \\
\end{array}$ & N/A & 1.4 , Table 1.1 & $\begin{array}{r}\text { Waste Stream } \\
\text { Eliminated } \\
\end{array}$ \\
\hline SR-W022 & DWPF Benzene & $\begin{array}{r}\text { Waste Stream } \\
\text { Eliminated }\end{array}$ & N/A & 1.4, Table 1.1 & $\begin{array}{r}\text { Waste Stream } \\
\text { Eliminated }\end{array}$ \\
\hline SR-W023 & $\begin{array}{c}\text { Cadmium } \\
\text { Safety/Control Rods }\end{array}$ & $\begin{array}{c}\text { Macroencapsulation in a } \\
\text { Cask as a } \\
\text { 90-Day Generator }\end{array}$ & N/A & $\begin{array}{r}\text { Appendix to } \\
\text { Chapter } 3\end{array}$ & $\begin{array}{c}\text { Complies with } \\
\text { LDR }\end{array}$ \\
\hline SR-W024 & $\begin{array}{c}\text { Mercury/Tritium Gold } \\
\text { Traps }\end{array}$ & $\begin{array}{l}\text { Meets LDR Treatment } \\
\text { Standard }\end{array}$ & N/A & $\begin{array}{l}\text { Appendix to } \\
\text { Chapter } 3\end{array}$ & $\begin{array}{l}\text { Complies with } \\
\text { LDR }\end{array}$ \\
\hline SR-W025 & $\begin{array}{l}\text { Solvent/TRU Job } \\
\text { Control Waste } \\
<100 \mathrm{nCi} / \mathrm{g}\end{array}$ & $\begin{array}{l}\text { Consolidated with SR- } \\
\text { W027* }\end{array}$ & N/A* & 1.4, Table $1.1^{*}$ & $\begin{array}{c}\text { Consolidated } \\
\text { with SR-W027* }\end{array}$ \\
\hline SR-W026 & CH Mixed TRU/Thirds & $\begin{array}{l}\text { Characterization at } \\
\text { SRS-WIPP Disposal }\end{array}$ & 4.1 & 4.2 .1 .1 & $\begin{array}{l}\text { Awaiting } \\
\text { Treatment }\end{array}$ \\
\hline SR-W027 & $\begin{array}{c}\text { CH Mixed TRU/F- } \\
\text { Listed Solvents }\end{array}$ & $\begin{array}{l}\text { Characterization at } \\
\text { SRS-WIPP Disposal }\end{array}$ & 4.1 & 4.2 .1 .1 & $\begin{array}{l}\text { Awaiting } \\
\text { Treatment }\end{array}$ \\
\hline SR-W028 & Mark 15 Filter Paper & $\begin{array}{c}\text { Combustion at } \\
\text { Commercial Facility or } \\
\text { Macroencapsulation or } \\
\text { Stabilization at } \\
\text { Commercial Facility } \\
\end{array}$ & N/A & 3.5 .1 & $\begin{array}{l}\text { Waste Stream } \\
\text { Treated }\end{array}$ \\
\hline SR-W029 & $\begin{array}{c}\text { M-Area Sludge } \\
\text { Treatability Samples }\end{array}$ & Waste Stream Treated & N/A & 3.5 .3 & $\begin{array}{l}\text { Waste Stream } \\
\text { Treated }\end{array}$ \\
\hline SR-W030 & $\begin{array}{l}\text { Spent Methanol } \\
\text { Solution }\end{array}$ & $\begin{array}{l}\text { Consolidated with SR- } \\
\text { W001 }\end{array}$ & N/A & 1.4, Table 1.1 & $\begin{array}{c}\text { Consolidated } \\
\text { with SR-W001 }\end{array}$ \\
\hline SR-W031 & $\begin{array}{l}\text { Uranium/Chromium } \\
\text { Solution }\end{array}$ & $\begin{array}{c}\text { Stabilization by } \\
\text { Vitrification-M-Area } \\
\text { Vendor Treatment } \\
\text { Facility } \\
\end{array}$ & N/A & 3.5 .3 & $\begin{array}{l}\text { Waste Stream } \\
\text { Treated }\end{array}$ \\
\hline SR-W032A & $\begin{array}{c}\text { Mercury-Contaminated } \\
\text { Heavy Water }\end{array}$ & $\begin{array}{l}\text { Waste Stream } \\
\text { Eliminated }\end{array}$ & $\mathrm{N} / \mathrm{A}$ & 3.5 .2 & $\begin{array}{l}\text { Treated to meet } \\
\text { LDR }\end{array}$ \\
\hline SR-W032B & $\begin{array}{l}\text { Mercury-Contaminated } \\
\text { Heavy Water Residues }\end{array}$ & $\begin{array}{c}\text { Solidification in } \\
\text { container as a 90-day } \\
\text { generator }\end{array}$ & N/A & $\begin{array}{l}\text { Appendix to } \\
\text { Chapter } 3\end{array}$ & $\begin{array}{l}\text { Complies with } \\
\text { LDR }\end{array}$ \\
\hline SR-W033 & $\begin{array}{l}\text { Thirds/TRU Job } \\
\text { Control Waste } \\
<100 \mathrm{nCi} / \mathrm{g}\end{array}$ & $\begin{array}{l}\text { Consolidated with SR- } \\
\text { W026* }\end{array}$ & N/A* & 1.4, Table $1.1^{*}$ & $\begin{array}{c}\text { Consolidated } \\
\text { with SR-W026* }\end{array}$ \\
\hline SR-W034 & Calcium Metal & $\begin{array}{c}\text { Deactivation by } \\
\text { commercial Vendor }\end{array}$ & 3.1 .2 .7 & 3.1 .2 .7 & $\begin{array}{l}\text { Awaiting } \\
\text { Treatment }\end{array}$ \\
\hline
\end{tabular}


Table 1.1 User's Guide to Chapters 3, 4, and 5 (cont'd)

\begin{tabular}{|c|c|c|c|c|c|}
\hline $\begin{array}{c}\text { Waste Stream } \\
\text { No. }\end{array}$ & Waste Stream Name & Preferred Option (PO) & $\begin{array}{l}\text { Volume I Section } \\
\text { Identification }\end{array}$ & $\begin{array}{c}\text { Volume II } \\
\text { Section } \\
\text { Identification } \\
\end{array}$ & $\begin{array}{c}\text { Waste Stream } \\
\text { Status }\end{array}$ \\
\hline SR-W035 & $\begin{array}{l}\text { Mixed Waste Oil- } \\
\text { Sitewide }\end{array}$ & $\begin{array}{l}\text { Thermal Treatment at } \\
\text { Commercial Facility }\end{array}$ & 3.1 .2 .6 & 3.1 .2 .6 & $\begin{array}{c}\text { Ongoing } \\
\text { Treatment }\end{array}$ \\
\hline SR-W036 & $\begin{array}{l}\text { Tritiated Oil with } \\
\text { Mercury }\end{array}$ & $\begin{array}{c}\text { Treatment by Aging } \\
\text { followed by Combustion }\end{array}$ & N/A & 3.4 & $\begin{array}{l}\text { Awaiting } \\
\text { Treatment }\end{array}$ \\
\hline SR-W037 & $\begin{array}{l}\text { M-Area Plating Line } \\
\text { Sludges }\end{array}$ & $\begin{array}{c}\text { Stabilization by } \\
\text { Vitrification M-Area } \\
\text { Vendor Treatment } \\
\text { Facility }\end{array}$ & N/A & 3.5 .3 & $\begin{array}{l}\text { Waste Stream } \\
\text { Treated }\end{array}$ \\
\hline SR-W038 & $\begin{array}{c}\text { Plating Line Sump } \\
\text { Material }\end{array}$ & $\begin{array}{c}\text { Stabilization by } \\
\text { Vitrification M-Area } \\
\text { Vendor Treatment } \\
\text { Facility }\end{array}$ & N/A & 3.5 .3 & $\begin{array}{c}\text { Waste Stream } \\
\text { Treated }\end{array}$ \\
\hline SR-W039 & $\begin{array}{l}\text { Nickel Plating Line } \\
\text { Solution }\end{array}$ & $\begin{array}{c}\text { Stabilization by } \\
\text { Vitrification M-Area } \\
\text { Vendor Treatment } \\
\text { Facility }\end{array}$ & N/A & 3.5 .3 & $\begin{array}{l}\text { Waste Stream } \\
\text { Treated }\end{array}$ \\
\hline SR-W040 & \begin{tabular}{c|} 
Listed Stabilized \\
Sludge/Listed LDR \\
- Compliant Debris
\end{tabular} & $\begin{array}{c}\text { Waste Stream } \\
\text { Treated in } \\
\text { Compliance with } \\
\text { LDR } \\
\end{array}$ & N/A & $\begin{array}{l}\text { Appendix to } \\
\text { Chapter } 3\end{array}$ & $\begin{array}{l}\text { Complies } \\
\text { with LDR }\end{array}$ \\
\hline SR-W041 & $\begin{array}{l}\text { Aqueous Mercury } \\
\text { and Lead }\end{array}$ & $\begin{array}{c}\text { Effluent Treatment } \\
\text { Project }\end{array}$ & N/A & $\begin{array}{l}\text { Appendix to } \\
\text { Chapter } 3\end{array}$ & $\begin{array}{l}\text { Treated to } \\
\text { meet LDR }\end{array}$ \\
\hline SR-W042 & Paints and Thinners & $\begin{array}{l}\text { Thermal Treatment } \\
\text { Macroencapsulation } \\
\text { or Stabilization at } \\
\text { Commercial Facility }\end{array}$ & 3.1 .2 .6 & 3.1 .2 .6 & $\begin{array}{c}\text { Ongoing } \\
\text { Treatment }\end{array}$ \\
\hline SR-W043 & $\begin{array}{l}\text { Lab Waste with } \\
\text { Tetraphenyl Borate }\end{array}$ & $\begin{array}{l}\text { Consolidated with } \\
\text { SR-W012 }\end{array}$ & N/A & 1.4 , Table 1.1 & $\begin{array}{c}\text { Consolidated } \\
\text { with SR-W012 }\end{array}$ \\
\hline SR-W044 & $\begin{array}{c}\text { Tri-Butyl- } \\
\text { Phosphate \& n- } \\
\text { Paraffin-TRU }\end{array}$ & $\begin{array}{l}\text { Consolidated with } \\
\text { SR-W045A \& B }\end{array}$ & $\mathrm{N} / \mathrm{A}$ & 1.4 , Table 1.1 & $\begin{array}{c}\text { Consolidated } \\
\text { with SR-W045 }\end{array}$ \\
\hline SR-W045A & $\begin{array}{c}\text { PUREX Aqueous } \\
\text { Waste }\end{array}$ & $\begin{array}{l}\text { Waste Stream } \\
\text { Eliminated }\end{array}$ & N/A & 1.4, Table 1.1 & $\begin{array}{c}\text { Waste } \\
\text { Stream } \\
\text { Eliminated }\end{array}$ \\
\hline SR-W045B & $\begin{array}{l}\text { PUREX Organic } \\
\text { Waste }\end{array}$ & $\begin{array}{c}\text { Waste Stream } \\
\text { renamed SR-W045 }\end{array}$ & N/A & 1.4, Table 1.1 & $\begin{array}{c}\text { Waste } \\
\text { Stream } \\
\text { renamed } \\
\text { SR-W045 }\end{array}$ \\
\hline SR-W045 & PUREX Waste & $\begin{array}{c}\text { Solidification using } \\
\text { new technology }\end{array}$ & 3.2 .3 & 3.2 .3 & $\begin{array}{l}\text { Awaiting } \\
\text { Treatment }\end{array}$ \\
\hline SR-W046 & $\begin{array}{l}\text { Consolidated } \\
\text { Incineration } \\
\text { Facility Ash }\end{array}$ & $\begin{array}{c}\text { Waste stream } \\
\text { eliminated }\end{array}$ & N/A & $\begin{array}{l}\text { Appendix to } \\
\text { Chapter } 3\end{array}$ & $\begin{array}{l}\text { Waste Stream } \\
\text { Treated }\end{array}$ \\
\hline SR-W047 & $\begin{array}{c}\text { Consolidated } \\
\text { Incineration Facility } \\
\text { Blowdown }\end{array}$ & Waste stream eliminated & N/A & $\begin{array}{l}\text { Appendix to } \\
\text { Chapter } 3\end{array}$ & $\begin{array}{l}\text { Waste Stream } \\
\text { Treated }\end{array}$ \\
\hline SR-W048 & $\begin{array}{l}\text { Soils from Spill } \\
\text { Remediation }\end{array}$ & $\begin{array}{c}\text { Stabilization by } \\
\text { Commercial Vendor }\end{array}$ & 3.1 .2 .5 & 3.1 .2 .5 & $\begin{array}{c}\text { Ongoing } \\
\text { Treatment }\end{array}$ \\
\hline SR-W049 & $\begin{array}{c}\text { Tank E-3-1 Clean Out } \\
\text { Material }\end{array}$ & Waste stream eliminated & N/A & 1.4 , Table 1.1 & $\begin{array}{l}\text { Waste Stream } \\
\text { Treated }\end{array}$ \\
\hline SR-W050 & $\begin{array}{c}\text { Waste to Support High- } \\
\text { Level Waste (HLW) } \\
\text { Processing } \\
\text { Demonstrations }\end{array}$ & $\begin{array}{c}\text { Treatment by SRNL as a } \\
\text { 90-Day Generator }\end{array}$ & N/A & $\begin{array}{l}\text { Appendix to } \\
\text { Chapter } 3\end{array}$ & $\begin{array}{l}\text { Complies with } \\
\text { LDR }\end{array}$ \\
\hline
\end{tabular}


Table 1.1 User's Guide to Chapters 3, 4, and 5 (cont'd)

\begin{tabular}{|c|c|c|c|c|c|}
\hline $\begin{array}{c}\text { Waste Stream } \\
\text { No. }\end{array}$ & $\begin{array}{l}\text { Waste Stream } \\
\text { Name }\end{array}$ & $\begin{array}{l}\text { Preferred Option } \\
\text { (PO) }\end{array}$ & $\begin{array}{l}\text { Volume I Section } \\
\text { Identification }\end{array}$ & $\begin{array}{c}\text { Volume II } \\
\text { Section } \\
\text { Identification } \\
\end{array}$ & $\begin{array}{c}\text { Waste Stream } \\
\text { Status }\end{array}$ \\
\hline SR-W051 & $\begin{array}{c}\text { Spent Filter Cartridges } \\
\text { and Carbon Filter } \\
\text { Media }\end{array}$ & $\begin{array}{l}\text { Thermal Treatment, } \\
\text { Macroencapsulation or } \\
\text { Stabilization and/or } \\
\text { alternative debris } \\
\text { treatment at Commercial } \\
\text { Facility }\end{array}$ & 3.1 .2 .6 & 3.1 .2 .6 & $\begin{array}{l}\text { Awaiting } \\
\text { Treatment }\end{array}$ \\
\hline SR-W052 & $\begin{array}{c}\text { Cadmium- } \\
\text { Contaminated } \\
\text { Glovebox Section }\end{array}$ & $\begin{array}{l}\text { Waste Stream } \\
\text { Eliminated }\end{array}$ & N/A & 1.4, Table 1.1 & $\begin{array}{l}\text { Waste Stream } \\
\text { Eliminated }\end{array}$ \\
\hline SR-W053 & Rocky Flats Ash & $\begin{array}{l}\text { Characterization at } \\
\text { SRS-Ship to WIPP }\end{array}$ & 4.2 .1 & 4.2 .2 & $\begin{array}{l}\text { Awaiting } \\
\text { Treatment }\end{array}$ \\
\hline SR-W054 & $\begin{array}{c}\text { Enriched Uranium } \\
\text { Contaminated with } \\
\text { Lead }\end{array}$ & $\begin{array}{l}\text { Consolidated with } \\
\text { SR-W037 }\end{array}$ & N/A & 1.4, Table 1.1 & $\begin{array}{c}\text { Consolidated } \\
\text { with SR-W037 }\end{array}$ \\
\hline SR-W055 & $\begin{array}{c}\text { Job Control Waste } \\
\text { Containing Solvent } \\
\text { Contaminated Wipes }\end{array}$ & $\begin{array}{l}\text { Thermal Treatment, } \\
\text { Macroencapsulation or } \\
\text { Stabilization at } \\
\text { Commercial Facility }\end{array}$ & 3.1 .2 .6 & 3.1 .2 .6 & $\begin{array}{c}\text { Ongoing } \\
\text { Treatment }\end{array}$ \\
\hline SR-W056 & $\begin{array}{c}\text { Job Control Waste } \\
\text { with Enriched Uranium } \\
\text { and Solvent } \\
\text { Contaminated Wipes }\end{array}$ & $\begin{array}{c}\text { Waste Stream } \\
\text { Re-characterized }\end{array}$ & N/A & 1.4, Table 1.1 & $\begin{array}{l}\text { Waste Stream } \\
\text { Eliminated }\end{array}$ \\
\hline SR-W057 & $\begin{array}{c}\text { D-Tested Neutron } \\
\text { Generators }\end{array}$ & $\begin{array}{l}\text { Waste Stream } \\
\text { Eliminated }\end{array}$ & $\mathrm{N} / \mathrm{A}$ & $\begin{array}{c}1.4, \text { Table } \\
1.1 \\
\end{array}$ & $\begin{array}{l}\text { Waste Stream } \\
\text { Eliminated } \\
\end{array}$ \\
\hline SR-W058 & $\begin{array}{c}\text { Mixed Sludge Waste } \\
\text { with Mercury from } \\
\text { DWPF Treatability } \\
\text { Studies }\end{array}$ & $\begin{array}{l}\text { Treatment by SRNL as } \\
\text { a 90-Day Generator }\end{array}$ & $\mathrm{N} / \mathrm{A}$ & $\begin{array}{l}\text { Appendix to } \\
\text { Chapter } 3\end{array}$ & $\begin{array}{l}\text { Treated to meet } \\
\text { LDR }\end{array}$ \\
\hline SR-W059 & $\begin{array}{c}\text { Tetrabutyl Titanate } \\
\text { (TBT) }\end{array}$ & $\begin{array}{l}\text { Consolidated with SR- } \\
\text { W001 }\end{array}$ & N/A & $\begin{array}{c}1.4, \\
\text { Table } 1.1\end{array}$ & $\begin{array}{c}\text { Consolidated } \\
\text { with SR-W001 }\end{array}$ \\
\hline SR-W060 & $\begin{array}{c}\text { Tritiated Water with } \\
\text { Mercury }\end{array}$ & $\begin{array}{l}\text { Macroencapsulation in } \\
\text { a Steel Container via a } \\
\text { Treatability Variance }\end{array}$ & 3.1 .1 .3 & 3.1 .1 .3 & $\begin{array}{l}\text { Awaiting } \\
\text { Variance } \\
\text { Approval }\end{array}$ \\
\hline SR-W061 & DWPF Mercury & $\begin{array}{l}\text { Consolidated with SR- } \\
\text { W068 }\end{array}$ & N/A & $\begin{array}{c}1.4, \\
\text { Table } 1.1 \\
\end{array}$ & $\begin{array}{c}\text { Consolidated } \\
\text { with SR-W068 }\end{array}$ \\
\hline SR-W062 & $\begin{array}{c}\text { Normal Low-Level } \\
\text { Contaminated Debris }\end{array}$ & $\begin{array}{l}\text { Macroencapsulation or } \\
\text { stabilization by a } \\
\text { commercial vendor } \\
\text { and/or alternative } \\
\text { debris treatment at an } \\
\text { offsite facility }\end{array}$ & 3.1 .2 .2 & 3.1 .2 .2 & $\begin{array}{c}\text { Ongoing } \\
\text { Treatment }\end{array}$ \\
\hline SR-W063 & $\begin{array}{c}\text { Macroencapsulated } \\
\text { Low-Level Waste } \\
\text { (TC) }\end{array}$ & $\begin{array}{l}\text { Meets Treatment } \\
\text { Standard }\end{array}$ & N/A & $\begin{array}{l}\text { Appendix to } \\
\text { Chapter } 3\end{array}$ & $\begin{array}{l}\text { Complies with } \\
\text { LDR }\end{array}$ \\
\hline SR-W064 & $\begin{array}{c}\text { IDW Soils/Sludges/ } \\
\text { Slurries }\end{array}$ & Awaiting ROD, etc. & N/A & 6.1 & $\begin{array}{c}\text { Future } \\
\text { Generation }\end{array}$ \\
\hline SR-W065 & $\begin{array}{c}\text { IDW Monitoring } \\
\text { Well } \\
\text { Purge/Development } \\
\text { Water }\end{array}$ & Awaiting ROD, etc. & N/A & 6.1 & $\begin{array}{c}\text { Future } \\
\text { Generation }\end{array}$ \\
\hline
\end{tabular}




\begin{tabular}{|c|c|c|c|c|c|}
\hline SR-W066 & IDW Debris & Awaiting ROD, etc. & N/A & 6.1 & $\begin{array}{c}\text { Future } \\
\text { Generation }\end{array}$ \\
\hline
\end{tabular}

Table 1.1 User's Guide to Chapters 3, 4, and 5 (cont'd)

\begin{tabular}{|c|c|c|c|c|c|}
\hline $\begin{array}{c}\text { Waste Stream } \\
\text { No. }\end{array}$ & $\begin{array}{l}\text { Waste Stream } \\
\text { Name }\end{array}$ & $\begin{array}{l}\text { Preferred Option } \\
\text { (PO) }\end{array}$ & $\begin{array}{l}\text { Volume I Section } \\
\text { Identification }\end{array}$ & $\begin{array}{c}\text { Volume II } \\
\text { Section } \\
\text { Identification } \\
\end{array}$ & $\begin{array}{c}\text { Waste Stream } \\
\text { Status }\end{array}$ \\
\hline SR-W067 & $\begin{array}{c}\text { IDW Personal } \\
\text { Protective } \\
\text { Equipment (PPE) } \\
\text { Waste }\end{array}$ & Awaiting ROD, etc. & N/A & 6.1 & $\begin{array}{c}\text { Future } \\
\text { Generation }\end{array}$ \\
\hline SR-W068 & $\begin{array}{l}\text { Elemental (Liquid) } \\
\text { Mercury-Sitewide }\end{array}$ & $\begin{array}{l}\text { Consolidated with SR- } \\
\text { W014* }\end{array}$ & N/A* & 1.4 , Table $1.1^{*}$ & $\begin{array}{c}\text { Consolidated } \\
\text { with SR-W014* }\end{array}$ \\
\hline SR-W069 & $\begin{array}{l}\text { Low-Level Waste } \\
\text { (LLW) Lead - To } \\
\text { be Macro- } \\
\text { encapsulated } \\
\text { Offsite }\end{array}$ & $\begin{array}{l}\text { Macroencapsulation by } \\
\text { a commercial vendor at } \\
\text { an offsite facility }\end{array}$ & 3.1 .2 .3 & 3.1 .2 .3 & $\begin{array}{l}\text { Ongoing } \\
\text { Treatment }\end{array}$ \\
\hline SR-W070 & $\begin{array}{l}\text { Mixed Waste from } \\
\text { Laboratory Samples }\end{array}$ & $\begin{array}{c}\text { Combustion at } \\
\text { Commercial Vendor } \\
\text { Facility or } \\
\text { Macroencapsulation or } \\
\text { Stabilization at } \\
\text { Commercial Vendor } \\
\text { Facility } \\
\end{array}$ & 3.1 .2 .6 & 3.1 .2 .6 & $\begin{array}{l}\text { Awaiting } \\
\text { Treatment }\end{array}$ \\
\hline SR-W071 & $\begin{array}{c}\text { Wastewater } \\
\text { Suitable for Thermal } \\
\text { treatment or } \\
\text { Stabilization } \\
\end{array}$ & $\begin{array}{l}\text { Thermal Treatment or } \\
\text { stabilization at } \\
\text { Commercial Vendor } \\
\text { Facility } \\
\end{array}$ & 3.1 .2 .6 & 3.1 .2 .6 & $\begin{array}{l}\text { Ongoing } \\
\text { Treatment }\end{array}$ \\
\hline SR-W072 & \begin{tabular}{|c|} 
Supernate or \\
Sludge \\
Contaminated \\
Debris from High- \\
Level Waste (HLW) \\
Operations \\
\end{tabular} & $\begin{array}{c}\text { Extraction or } \\
\text { Immobilization } \\
\text { Alternative Debris } \\
\text { Technologies as a 90- } \\
\text { Day Generator }\end{array}$ & N/A & $\begin{array}{l}\text { Appendix to } \\
\text { Chapter } 3\end{array}$ & $\begin{array}{l}\text { Complies with } \\
\text { LDR }\end{array}$ \\
\hline SR-W073 & $\begin{array}{c}\text { Cadmium- } \\
\text { Containing Raschig } \\
\text { Rings }\end{array}$ & $\begin{array}{l}\text { Macroencapsulation by } \\
\text { Commercial Vendor } \\
\text { Offsite via Treatability } \\
\text { Variance or } \\
\text { stabilization at } \\
\text { Commercial Vendor } \\
\text { Facility }\end{array}$ & 3.1 .2 .2 & 3.1 .2 .2 & $\begin{array}{l}\text { Awaiting } \\
\text { Treatment }\end{array}$ \\
\hline SR-W077 & $\begin{array}{c}\text { Aqueous } \\
\text { Characteristic } \\
\text { Wastewater }\end{array}$ & $\begin{array}{l}\text { Ion Exchange, Filtration, } \\
\text { nd/or Stabilization at F/H } \\
\text { ETP, Saltstone, or } \\
\text { Commercial Vendor }\end{array}$ & N/A & $\begin{array}{l}\text { Appendix to } \\
\text { Chapter } 3\end{array}$ & $\begin{array}{l}\text { Ongoing } \\
\text { Treatment }\end{array}$ \\
\hline SR-W078 & $\begin{array}{l}\text { LDR Hazardous } \\
\text { Waste Awaiting } \\
\text { Radiological } \\
\text { Screening }\end{array}$ & $\begin{array}{l}\text { Waste Stream } \\
\text { Eliminated }\end{array}$ & 3.3 .2 & 3.3 .2 & $\begin{array}{l}\text { Waste Stream } \\
\text { Eliminated }\end{array}$ \\
\hline SR-W079 & $\begin{array}{l}\text { Polychlorinated } \\
\text { Biphenyl (PCB) } \\
\text { Mixed Waste }\end{array}$ & $\begin{array}{l}\text { Combustion at the } \\
\text { ETTP TSCA } \\
\text { Incinerator or vendor } \\
\text { alternate debris } \\
\text { technology treatment }\end{array}$ & 3.1 .2 .1 & 3.1 .2 .1 & $\begin{array}{l}\text { Ongoing } \\
\text { Treatment }\end{array}$ \\
\hline
\end{tabular}




\begin{tabular}{|c|c|c|c|c|c|}
\hline $\begin{array}{c}\text { SR-W080* } \\
(\mathrm{CN}-W 001, \mathrm{CN}- \\
\text { W004) }\end{array}$ & $\begin{array}{c}\text { Charleston Naval } \\
\text { Shipyard Waste }\end{array}$ & $\begin{array}{c}\text { Thermal Treatment or } \\
\text { Macroencapsulation or } \\
\text { Stabilization at } \\
\text { Commercial Vendor }\end{array}$ & 3.1 .2 .6 & 3.1 .2 .6 & $\begin{array}{c}\text { Awaiting } \\
\text { Treatment }\end{array}$ \\
\hline
\end{tabular}

Table 1.1 User's Guide to Chapters 3, 4, and 5 (cont'd)

\begin{tabular}{|c|c|c|c|c|c|}
\hline $\begin{array}{c}\text { Waste Stream } \\
\text { No. }\end{array}$ & $\begin{array}{l}\text { Waste Stream } \\
\text { Name }\end{array}$ & $\begin{array}{l}\text { Preferred Option } \\
\text { (PO) }\end{array}$ & $\begin{array}{l}\text { Volume I Section } \\
\text { Identification }\end{array}$ & $\begin{array}{c}\text { Volume II } \\
\text { Section } \\
\text { Identification } \\
\end{array}$ & $\begin{array}{c}\text { Waste Stream } \\
\text { Status }\end{array}$ \\
\hline SR-W081 & $\begin{array}{l}\text { Reactive/Ignitable } \\
\text { Waste }\end{array}$ & $\begin{array}{c}\text { Deactivation } \\
\text { followed by } \\
\text { stabilization or } \\
\text { combustion at } \\
\text { Commercial Vendor }\end{array}$ & 3.1 .2 .6 & 3.1 .2 .6 & $\begin{array}{c}\text { Ongoing } \\
\text { Treatment }\end{array}$ \\
\hline SR-W082 & $\begin{array}{c}\text { Radioactive } \\
\text { Chemicals, Metals, } \\
\text { and Pesticides (CMP) } \\
\text { Soil }\end{array}$ & $\begin{array}{c}\text { Stabilization by } \\
\text { Commercial Vendor }\end{array}$ & 3.1 .2 .5 & 3.1 .2 .5 & $\begin{array}{l}\text { Ongoing } \\
\text { Treatment }\end{array}$ \\
\hline SR-W083 & $\begin{array}{l}\text { Mercury- and } \\
\text { Chromium- } \\
\text { Contaminated } \\
\text { Residues } \\
\end{array}$ & $\begin{array}{l}\text { Waste Stream Re- } \\
\text { Characterized }\end{array}$ & N/A & 1.4, Table 1.1 & $\begin{array}{l}\text { Waste Stream } \\
\text { Eliminated }\end{array}$ \\
\hline SR-W084 & $\begin{array}{l}\text { Remediation Waste } \\
\text { Soils that Meet LDR }\end{array}$ & $\begin{array}{l}\text { Meets Treatment } \\
\text { Standard } \\
\end{array}$ & N/A & 1.4, Table 1.1 & $\begin{array}{c}\text { Complies with } \\
\text { LDR } \\
\end{array}$ \\
\hline SR-W085 & $\begin{array}{c}\text { Rocky Flats } \\
\text { Plutonium Fluoride } \\
\text { Residues } \\
\end{array}$ & $\begin{array}{l}\text { Future Generation- } \\
\text { Treatment to be } \\
\text { Determined } \\
\end{array}$ & N/A & 1.4, Table 1.1 & $\begin{array}{c}\text { Future } \\
\text { Generation }\end{array}$ \\
\hline SR-W086 & $\begin{array}{l}\text { Characteristically } \\
\text { Hazardous Non- } \\
\text { Incinerable Solids }\end{array}$ & $\begin{array}{c}\text { Amalgamation at } \\
\text { Commercial Vendor }\end{array}$ & 3.1 .2 .4 & 3.1 .2 .4 & $\begin{array}{l}\text { Awaiting } \\
\text { Treatment }\end{array}$ \\
\hline $\begin{array}{c}\text { SR-W087 } \\
\text { (BT-W035, part } \\
\text { of BT-W003) } \\
\end{array}$ & $\begin{array}{c}\text { Bettis Atomic Power } \\
\text { Laboratory } \\
\text { Contaminated Oil }\end{array}$ & $\begin{array}{l}\text { Thermal Treatment } \\
\text { or Stabilization at } \\
\text { Commercial Facility }\end{array}$ & 3.1 .2 .6 & 3.1 .2 .6 & $\begin{array}{l}\text { Awaiting } \\
\text { Treatment }\end{array}$ \\
\hline SR-W088 & $\begin{array}{l}\text { Sludges and Non- } \\
\text { Debris Solids* }\end{array}$ & $\begin{array}{c}\text { Stabilization by } \\
\text { Commercial Vendor }\end{array}$ & 3.1 .2 .5 & 3.1 .2 .5 & $\begin{array}{c}\text { Ongoing } \\
\text { Treatment }\end{array}$ \\
\hline SR-W089 & $\begin{array}{l}\text { TRU Mixed Waste } \\
\text { from Mound Site }\end{array}$ & $\begin{array}{c}\text { Characterization at } \\
\text { SRS - WIPP Disposal }\end{array}$ & 4.2 .2 & 4.2 .2 & $\begin{array}{c}\text { Awaiting } \\
\text { Treatment }\end{array}$ \\
\hline SR-W090 & $\begin{array}{l}\text { Elemental Mercury } \\
\text { - High Rad }\end{array}$ & $\begin{array}{c}\text { Treatment by aging } \\
\text { F/B mercury } \\
\text { treatment* }\end{array}$ & 3.4 & $3.4 *$ & $\begin{array}{l}\text { Awaiting } \\
\text { Treatment }\end{array}$ \\
\hline SR-W091* & $\begin{array}{c}\text { Contaminated } \\
\text { Debris (High Rad)* }\end{array}$ & $\begin{array}{l}\text { New Stream - } \\
\text { Treatment to be } \\
\text { Determined* } \\
\end{array}$ & N/A* & $3.1 .3^{*}$ & $\begin{array}{c}\text { Awaiting } \\
\text { Treatment* }\end{array}$ \\
\hline
\end{tabular}

Some waste streams may be only briefly mentioned if they have been treated to meet LDR standards, recharacterized, or consolidated. Only the waste streams that require a schedule and a compliance order will be found in the Compliance Plan Volume. Waste streams not found in the Compliance Plan Volume have been recharacterized, combined, or are in compliance with applicable regulations.

* Indicates that a change has been made since last update. 
THIS PAGE INTENTIONALLY LEFT BLANK. 


\section{Chapter 2. Key Order Provisions}

Implementation of the STP will be by SCDHEC Consent Order 95-22-HW (Order). The purpose of this chapter is to reiterate key provisions of the Order.

\subsection{Definitions}

a. Project Activity Schedule(s) shall mean the plan in the STP for performing key activities in support of mixed waste treatment(s). Project activity schedules will be provided in Chapters 3 through 5 of this Volume in accordance with the Section 3021(b)(1)(B)(ii) of the FFCAct.

b. Milestone(s) shall mean those specific date(s) or time frame(s) within the STP project activity schedule(s) that constitute the steps DOE-SR is committing to take to provide for treatment of its mixed waste.

c. Cleanup Credits shall mean activities performed by DOE as identified in the Statement of Mutual Understanding "Site Treatment Plan Cleanup Credit."

d. Day(s) are defined as calendar days; activities defined as occurring within a given quarter shall be completed by the last day of the quarter.

e. Revision(s) shall mean a change to the STP, which includes but is not limited to the addition of a treatment facility, treatment capacity, or technology development not previously included in this Compliance Plan Volume.

f. Modification(s) shall mean a change to the STP that does not constitute a revision.

g. Mixed Waste(s) shall mean waste that contains both hazardous wastes and source, special nuclear, or byproduct materials, subject to the Atomic Energy Act of 1954 (42 2011 U.S.C. et seq.).

h. Fiscal Year (FY) shall mean the federal fiscal year, which begins October 1 and ends September 30.

\subsection{Project Activity Schedules}

The schedules identified in Chapters 3, 4, and 5 represent DOE's plan for treating the Site's mixed waste. Changes to these schedules require SCDHEC approval. Appendix A represents those schedule activities that occur in the upcoming federal fiscal year and that DOE agrees are enforceable commitments unless otherwise proposed by DOE and approved by SCDHEC. Cleanup Credits, as established in the Statement of Mutual Understanding- Site Treatment Plan Cleanup Credits, may be used to achieve compliance with enforceable STP commitments. Appendix B represents those schedule activities planned to occur in the subsequent two federal fiscal years. During the STP annual update process, Chapters 3, 4, and 5 schedule activities will be moved into Appendix B, and Appendix B activities will be moved to Appendix A as scheduled unless otherwise proposed by DOE and approved by SCDHEC.

During the annual budget planning process, DOE-SR will seek funding by submitting a target budget request and identifying any additional funding required to accomplish activities identified in Appendix B as occurring in the upcoming federal fiscal year plus one. Additionally, DOE-SR will evaluate the funding status of the activities identified in Appendix B as occurring in the upcoming federal fiscal year plus two and those activities identified in Appendix A.

If a funding shortfall is identified for Appendix A or B activities, DOE-SR shall notify SCDHEC and attempt to resolve the shortfall through obtaining additional funds, reprioritization, and/or implementing improved operating efficiencies. If the funding shortfall for Appendix A is not resolved, DOE-SR will request a schedule modification or revision, as appropriate.

During the budgeting process, DOE-SR will also evaluate schedule activities beyond the upcoming federal fiscal year plus the next two federal fiscal years to identify required funding. If shortfalls are identified, DOESR shall notify SCDHEC and attempt to resolve the shortfall through reprioritization and/or implementing improved operating efficiencies. If the funding shortfall is not resolved, DOE-SR may request a schedule modification or revision, as appropriate. 


\subsection{Covered Matters}

\section{Applicability}

Except as specifically set forth elsewhere in this plan, this plan shall apply to the RCRA LDR requirements pertaining to past, ongoing, and future generation, storage, and treatment of mixed waste at SRS, the hazardous component of which is subject to the LDR. LDR requirements can be found in the South Carolina Hazardous Waste Management Regulations (SCHWMR) R.61-79.268 and the Code of Federal Regulations, Chapter 40, Part 268.

\section{Mixed Waste Treatment}

This plan addresses the development of treatment capacities and technologies for treating or otherwise managing SRS mixed wastes in accordance with RCRA LDR, regardless of the time when the mixed waste was generated. For the purpose of this plan, covered mixed waste shall mean that mixed waste not excluded by the Covered Matters herein.

\section{Exclusions-General}

Inasmuch as the intent of the FFCAct is to develop an STP to address compliance with RCRA Section 3004(j), this Compliance Plan Volume shall not address mixed waste being stored or generated at SRS which (1) meets LDR requirements, regardless of when generated; or (2) mixed wastes that are being stored, or will be stored, when generated, solely for the purpose of accumulating sufficient quantities of mixed wastes as are necessary to facilitate proper recovery, treatment, or disposal in accordance with SCHWMR 61.-79.268.50. Information pertaining to the status of the mixed waste described above is provided in the Background Volume of this STP. By previous agreement with SCDHEC, small (less than 55 gallons) quantities of mixed waste(s) stored in RCRA Satellite Accumulation Areas [R.61-79.262.34(c)] are not subject to R.61-79.268 and are not included in this plan, unless requested otherwise by SCDHEC.

\section{RCRA/Comprehensive Environmental Response Compensation and Liability Act (CERCLA)}

Corrective actions and response actions shall be addressed by the Comprehensive Environmental Response Compensation and Liability Act (CERCLA) Section 120 Federal Facility Agreement (FFA) that was negotiated by EPA, DOE-SR, and SCDHEC (effective date August 16, 1993), any RCRA hazardous waste permits issued or to be issued by the State of South Carolina and EPA, orders issued pursuant to Section 3008(h) of RCRA, and/or by an agreement, order, or legal action under CERCLA. SCDHEC and DOE-SR acknowledge that this plan does not address mixed waste subject to corrective actions pursuant to RCRA and response actions pursuant to CERCLA, unless waste is removed from the area of contamination and not otherwise subject to the provisions of the RCRA/CERCLA orders or agreements.

\section{Environmental Restoration}

This plan excludes (1) environmental restoration mixed wastes derived from RCRA corrective actions and CERCLA response actions that do not involve the land disposal of hazardous wastes (e.g., the placement of remediation wastes into or within a corrective action management unit or area of contamination) and/or (2) mixed waste for which a specific treatment path is included in another existing regulatory agreement (e.g., FFA, mixed aqueous Investigation Derived Waste (IDW) in the SRS IDW Management Plan, or mixed waste with a designated treatment listed in Records of Decision (RODs)/orders), permit or order or modifications thereof. Other environmental restoration mixed waste streams not specifically excluded will be dispositioned in accordance with the strategy provided in Volume II, Section 6.1. Information on any mixed waste for which SRS proposes to be excluded from the STP shall be submitted to SCDHEC for approval. 


\section{Compliance Issues}

This plan does not address RCRA compliance issues other than those issues specifically addressed herein. Therefore, SCDHEC and DOE-SR acknowledge that this plan does not affect the rights of SCDHEC to address any RCRA violations that exist or may exist at SRS not specifically covered by this plan.

\subsection{Funding}

\section{Process}

DOE-SR shall use its best efforts, in accordance with the DOE federal appropriations process, to request timely funding to meet its obligations under this plan.

\section{Anti-Deficiency Act}

No provision herein shall be interpreted to require obligation or payment of funds in violation of the AntiDeficiency Act, 31 U.S.C. $§ 1341$.

\subsection{Changes to STP}

\section{Annual Update}

SRS shall submit to the SCDHEC an Annual Update to the STP. This Annual Update shall be in compliance with Section 3021(b) of the FFCAct and shall include, but is not limited to, an updated inventory of all mixed waste, the status of all treatment residuals, and an updated implementation schedule. Projections of new mixed waste streams generated, or to be generated, onsite and proposed to be received from offsite shall be included in the Annual Update. A list of all proposed changes to the approved STP with a justification for requesting such changes shall be provided with the Annual Update. Unless otherwise notified by the SCDHEC, SRS shall not propose, in the Annual Update, modifications or revisions to the approved STP that have been previously denied by the SCDHEC.

\section{Modifications and Revisions}

SRS shall submit, for SCDHEC approval, a request for a modification or revision to Volume I of the approved STP for any change, unless the change requires notification only. (See Section 2.1 of Volume I for definitions of modification and revision.) All requests for modifications or revisions must meet the requirements of Section 3021(b) of the FFCAct. SRS may begin implementation of any modification or revision only upon receipt of written approval by the SCDHEC after appropriate public notice, if required. SCDHEC shall ensure that the public notice requirements of the FFCAct are addressed. The application of Cleanup Credits to an activity listed in Volume I of the STP is not defined as a modification or revision to the STP.

\section{Additional RCRA Permit Identification}

If SRS determines that treatment preparation steps, such as characterization, may require RCRA permits or a RCRA Interim Status Expansion, SRS will submit a revision or modification, as appropriate, to identify proposed permit application submittal dates to be included in Volume I project activity schedules.

\section{Alternate Treatment Strategy}

If SRS determines that a proposed treatment strategy is inappropriate, SRS will submit a revision or modification and identify the new proposed strategy. 
THIS PAGE INTENTIONALLY LEFT BLANK. 


\section{Chapter 3. Mixed Low-Level Waste Treatment}

The sections of this chapter discuss project activity schedules proposed for the treatment of mixed waste in accordance with Section 2.2 of this volume. Refer to Table 1.1 in Chapter 1 of this volume for the User's Guide to the waste streams in the STP. The User's Guide identifies each waste stream, the preferred treatment option (PO), and the location where the waste stream is discussed in Volumes I and II. The sections of this chapter are divided into treatment categories as follows:

3.1 Mixed Low-Level Waste Streams with Treatment Capacity

3.2 Mixed Low-Level Waste Streams Requiring Technology Development

3.3 Mixed Low-Level Waste Streams for Which Further Characterization is Required

3.4 Mixed Low-Level Waste Streams Requiring Radionuclide Decay Prior to LDR Treatment

3.5 Mixed Low-Level Waste Stream—Other Commitments

\subsection{Mixed Low-Level Waste Streams with Treatment Capacity}

This section discusses the schedules and plans associated with treatment of SRS mixed waste with currently available treatment capacity. This section of the chapter is divided into treatment categories as follows:

3.1.1 Onsite Treatment

3.1.2 Offsite Treatment

3.1.3 Preferred Treatment to be Determined

\subsubsection{Onsite Treatment}

SRS has several onsite treatment facilities for treating mixed low level waste. These facilities include the F and H Effluent Treatment Project (F/H ETP). Also included in this category are Treatability Variance Petition submittals, recycling, and Saltstone.

\subsubsection{F-Area and H-Area Effluent Treatment Project (F/H ETP)}

As waste characterizations are reviewed, aqueous characteristic wastes, such as those in SR-W041 and SRW077 may be treated at ETP if appropriate and approved. (See Section 9.1.1 of Volume II for more information.)

\subsubsection{Reserved.}

\subsubsection{Onsite Treatment Via Treatability Variance Submittals}

Submittal of a Treatability Variance for Macroencapsulation is the preferred option for certain mixed waste streams including the following:

SR-W009, Silver Coated Packing Material

SR-W060, Tritiated Water with Mercury

Estimated Schedule for Treatment of SR-W009

Submittal of applicable permit application(s):

Completed. Treatability Variance was submitted on September 18, 1997. (No RCRA permit modification will be required for performing this activity in a RCRA-permitted or interim status storage facility.) 
Entering into contracts:

Initiating construction:

Conducting systems testing:

Commencing operations:

Submitting waste processing schedule:
Initiate procurement within 3 months of approval of the treatability variance petition. Initiating

procurement shall mean issuing a request for proposal based on the approved treatability variance.

Initiate construction within 12 months of approval of the treatability variance petition. Initiate construction shall mean initiating equipment and procured materials installation.

Initiate systems testing within 6 months of initiating construction. Initiation of system testing shall mean beginning equipment checkout, developing procedures, and planning required self-assessments.

Commence operations within 6 months of initiating systems testing. Commence operations shall mean macroencapsulating mixed waste in accordance with the approved treatability variance.

Within 4 months after commencing operations, submit schedule for processing backlogged and currently generated mixed waste(s).

\section{Estimated Schedule for the Treatment of SR-W060}

Submit applicable permit application(s):

Completed. Treatability Variance for SR-W060 was submitted on September 18, 1997.

\section{$\underline{\text { Schedule Assumptions }}$}

The ability to perform in accordance with the estimated schedule depends on, but is not limited to, the following:

- Acceptable RCRA storage facility will be available when the treatability variance is approved,

- Receipt by DOE-SR of adequate funding specifically identified for this project to support the schedule,

- Completion of appropriate National Environmental Policy Act (NEPA) documentation,

- Resolution of any technically related finding(s) that might result from an operational readiness selfassessment or the systems testing phase,

- No changes in regulations, statutes, or the regulator's interpretations, and

- Schedule can be extended where good cause exists including, but is not limited to:

- circumstances unforeseen at the time the schedule was prepared that significantly affect the work required,

- delays in review of permit application(s), permit(s), or delays in approval of any other documents or other items needed to satisfy the requirements outlined,

- a delay caused by insufficient funding where DOE, in a timely manner and in good faith, requested adequate funding in accordance with the federal appropriations process, but Congress failed to appropriate such funding.

\subsubsection{Offsite Treatment}


For those waste streams that cannot be accommodated by SRS treatment facilities, various treatment technologies available at offsite facilities have been determined to be the preferred treatment option. This section discusses the waste streams that are available for offsite treatment. Offsite treatment includes offsite commercial vendor treatment and offsite treatment at other DOE facilities.

\subsubsection{DOE Thermal Treatment}

Combustion at the East Tennessee Technology Park (ETTP) TSCA Incinerator or vendor alternate debris technology treatment are the preferred options for the following waste stream:

SR-W079, Polychlorinated Biphenyl (PCB) Mixed Waste

Approved, equivalent treatment technologies offered by commercial vendors will be considered as they become available.

\section{Estimated Schedule for Treatment of This Waste Stream:}

Submit commitment schedule:

Completed. Schedule was submitted July 21, 1997. Schedule included the following commitments:

$-1^{\text {st }}$ Phase completed. Comments were resolved on the preliminary application and the formal application for the treatment of SR-W079 was submitted June 9, 1998 (required date 6/3/98). The Residuals Management Contingency Plan (RMCP) was approved by SCDHEC July 2, 1998, contingent upon agreement by the Tennessee Department of Environmental Conservation (TDEC).

$-2^{\text {nd }}$ Phase completed. A shipping and treatment schedule for the PCB mixed waste was submitted 7/16/99, which was within 90 days of receipt of written approval by the ETTP to accept the waste.

-3rd Phase completed. A shipment schedule for the PCB mixed waste was submitted 7/10/02 within 90 days of receipt of written approval by the state of Tennessee on 4/15/02 to accept the waste.

-4th Phase completed. Solid PCB mixed waste was shipped 7/30/02 (required date was 8/30/02). Liquid PCB mixed waste was shipped 9/26/01. Solid PCB MW currently in storage is being accumulated for future shipment. 


\section{Schedule Assumptions:}

The ability to perform in accordance with the estimated schedule for the treatment of this waste in the ETTP

TSCA Incinerator depends on, but is not limited to, the following:

- $\quad$ Receipt by DOE-SR of adequate funding identified for this project to support the schedule,

- $\quad$ Formal approval by the ETTP to ship the waste,

- Completion of the appropriate NEPA documentation,

- Agreement by the state regulatory agencies of South Carolina and Tennessee at least 60 days prior to the planned shipment date of the waste,

- $\quad$ SRS shipment of the waste stream according to the shipment schedule, and

- Waste treatment residuals will be sent to a commercial disposal facility or will be returned to SRS.

\subsubsection{Commercial Vendor Debris Treatment}

Alternate debris technology treatment, or macroencapsulation, or shredding followed by stabilization, at an offsite commercial vendor's facility are the preferred options for certain mixed waste streams, including the following:

SR-W062, Low-Level Contaminated Debris

\section{Estimated Schedule for Treatment of Waste Stream SR-W062}

Submittal of applicable permit application(s):

Issuing Request for Proposal:

Entering into contract(s):

Commencing operations:

Submitting waste processing schedule:
Offsite treatment; no SRS permits required.

Completed. The effectiveness of DOE's Broad Spectrum Contract Vendor was evaluated 2/20/01. No additional Request For Proposal is necessary.

Completed. The Broad Spectrum Contract Vendor was determined to be effective $2 / 20 / 01$ for this waste stream. No additional contracts are necessary.

Completed. Waste acceptance documentation (profile sheet) for the initial shipment was sent to the vendor June 27, 2001. (Required date February 20, 2002.)

Initial phase completed. First shipment was made September 27, 2001 (required date for first shipment schedule was November 22, 2001).

$2^{\text {nd }}$ Phase completed. $50 \%\left(78.4 \mathrm{~m}^{3}\right)$ of the remaining SR-W062 waste inventory (in RCRA storage buildings on 9/30/01) was shipped to vendor on 7/10/02 (required date for shipment was 9/30/02).

$3^{\text {rd }}$ phase completed. $100 \%$ of the remaining SR-W062 waste inventory (in RCRA storage buildings on 9/30/01) was shipped to the vendor by $9 / 15 / 04$, in addition to newly generated waste or waste that was transferred from other MWIR waste streams to SR-W062. Since 9/30/01, a total of $177.35 \mathrm{~m} 3$ of waste stream SR-W062 have been shipped for offsite treatment and disposal. Two B-25s of waste, which had originally been placed in this wastestream, were found to contain radiological contamination in excess of the vendor's license limits and have been placed in a new waste stream, SR-W091. 


\section{Schedule Assumptions}

The ability to perform in accordance with the estimated schedule for the Vendor treatment process depends on, but is not limited to, the following:

- $\quad$ Receipt by DOE-SR of adequate funding specifically identified for this project to support the schedule,

- Completion of appropriate NEPA documentation,

- Completion of upgrades to an existing SRS building to provide capabilities to sort, segregate, confirm characterization, and repackage waste for treatment,

- No changes in regulations, statutes, or the regulator's interpretations,

- Agreement by regulatory agencies of South Carolina and the vendor facility state regulatory agency at least 60 days prior to the planned shipment date,

- Waste treatment residues will be sent to a commercial disposal facility or will be returned to SRS.

- $\quad$ Schedule can be extended where good cause exists including, but is not limited to:

- circumstances unforeseen at the time the schedule was prepared that significantly affect the work required,

- delays in review of documents or other items needed to satisfy the requirements outlined,

- any other event or series of events including, but not limited to, the discovery of new technological information or technological barriers that significantly affects the work required, or

- a delay caused by insufficient funding where DOE, in a timely manner and in good faith, requested adequate funding in accordance with the federal appropriations process, but Congress failed to appropriate such funding, and

- Ability to complete confirmation of radiological characterization of the waste in an existing onsite RCRApermitted building to meet transportation requirements and offsite vendor's waste acceptance requirements.

\subsubsection{Commercial Vendor Lead Treatment}

Lead Macroencapsulation at a commercial vendor's facility is the preferred option for certain mixed waste streams, including but not limited to, the following:

SR-W069, Low-Level Waste (LLW) Lead - to be Macroencapsulated Offsite

Estimated Schedule for Treatment of Waste Streams SR-W069

Submit applicable permit application(s):

Issue Request for Proposal:

Enter into contract(s):

Waste Acceptance:
Completed. No treatability variance is necessary, based on EPA and SCDHEC concurrence that radioactive lead-acid batteries are considered "radioactive lead solids."

Completed. Request For Proposal issued 6/19/02 (required date 6/30/02).

Completed. Contract with a commercial vendor executed 12/10/02 (required date 12/31/02).

Completed. Waste profile sheets for the initial shipment to the offsite vendor were submitted 7/14/03 (due date $12 / 10 / 03)$. 
Submit waste processing schedule:

Phase I Completed. The initial shipment of waste to the offsite vendor was sent 9/18/03 (due date of shipment schedule was 11/05/03). Submit a schedule for shipping the remaining waste (in storage as of 2/1/03) within 24 months of contract execution (12/10/04).

\subsubsection{Commercial Vendor Mercury Treatment}

The preferred option is direct amalgamation (AMLGM)or stabilization by a commercial vendor, dependent upon vendor states' approval of direct AMALGM for the waste. The following waste streams are in this category:

SR-W014, Elemental (Liquid) Mercury - Sitewide

SR-W068, Elemental (Liquid) Mercury - Sitewide - Consolidated with SR-W014

SR-W086, Characteristically Hazardous Non-Incinerable Solids

\section{Estimated Schedule for Treatment of these Waste Streams}

Disposition of these waste streams is contingent upon completion of arrangements with a

Commercial vendor. See STP Volume II, Chapter 3, Section 3.1.2.4 for additional information.

Enter into contracts:

Phase I completed. Procurement established work agreement with Commercial Vendor 3/26/03 to treat streams SR-W014, SR-W068, and SR-W086 (due date 6/30/03).

Phase II completed. SRS provided waste characterization data to Commercial Vendor to assure conformance to vendor's waste acceptance criteria on 9/23/03 (due date 9/26/03).

Submit waste processing schedule:

Within 90 days of receipt of vendor approval of the waste, SRS will submit a shipping schedule for these waste streams.

\section{Schedule Assumptions}

The ability to perform the treatment process depends on, but is not limited to, the following:

- $\quad$ Receipt by DOE-SR of adequate funding identified for this project to support the schedule,

- Completion of appropriate NEPA documentation,

- Agreement by the regulatory agencies of South Carolina and contracting state at least 60 days prior to the planned shipment date of the waste.

- $\quad$ SRS shipment of the waste streams according to the shipment schedule, and

- Waste treatment residues will be shipped by the treatment vendor to a commercial disposal facility in conformance with LDR requirements, or will be returned to SRS.

\subsubsection{Commercial Vendor Solids Treatment}

The preferred treatment option can include several treatment steps. Wastes that contain organic material in excess of the LDR Treatment Standard are initially treated to separate the organic portion of the contaminants using solvent extraction or thermal separation. The desorbed chemical constituents are then treated via direct chemical oxidation or combustion. The waste material remaining after organics are removed or the non-organic waste material is then either directly stabilized, shredded and stabilized, macroencapsulated or subjected to alternative debris treatment technologies. No stabilization of organics is performed, and no impermissible dilution occurs. Wastes that do not contain significant organics are either directly stabilized, shredded and 
stabilized, macroencapsulated or subjected to alternative debris treatment technologies, as appropriate under RCRA regulations.

The following waste streams are included in this category:

SR-W048, Soils from Spill Remediation (stabilization thermal treatment)

SR-W073, Cadmium-Containing Raschig Rings (shredded and stabilized)

SR-W082, Radioactive Chemicals, Metals, and Pesticides Soils (stabilization thermal treatment)

SR-W088, Sludges and Non-Debris Solids (thermal treatment, stabilization)

\section{Estimated Schedule for Treatment of these Waste Streams}

Disposition of these waste streams is contingent upon completion of arrangements with a commercial vendor. See STP Volume II, Chapter 3, Section 3.1.2.5 for additional information.

Enter into contracts:

Submit waste processing schedule:
Phase I completed. Procurement established work agreement with Commercial Vendor 3/26/03 to treat streams SR-W048, SR-W073, SR-W082, and SR-W088 (due date 9/30/03). Phase II completed. SRS provided waste characterization data for streams SR-W048, SR-W082, and SR-W088 to Commercial Vendor to assure conformance to vendor's waste acceptance criteria on 9/22/03 (due date 9/26/03). Stream SR-W073 data submitted 5/21/04.

Within 90 days of vendor approval of the waste, SRS will submit a shipping schedule to SCDHEC for these waste streams (due date 11/23/04).

\section{$\underline{\text { Schedule Assumptions }}$}

The ability to perform the treatment process depends on, but it not limited to, the following:

- $\quad$ Receipt by DOE-SR of adequate funding identified for this project to support the schedule,

- Completion of appropriate NEPA documentation,

- Agreement by the regulatory agencies of South Carolina and contracting state at least 60 days prior to the planned shipment date of the waste.

- $\quad$ SRS shipment of the waste streams according to the shipment schedule, and

- Waste treatment residues will be shipped by the offsite treatment contractor to a commercial disposal facility in conformance with LDR requirements, or will be returned to SRS.

\subsubsection{Commercial Vendor "Non-PUREX" Treatment}

The preferred treatment option can include several treatment steps. Wastes that contain organic material in excess of the LDR Treatment Standard are initially treated to separate the organic portion of the contaminants using solvent extraction or thermal separation if necessary. The desorbed chemical constituents are then treated via direct chemical oxidation or combustion. The waste material remaining after organics are removed is then either directly stabilized, shredded and stabilized, macroencapsulated or subjected to alternative debris treatment technologies. No stabilization of organics is performed, and no impermissible dilution occurs.

The following waste streams are included in this category, with the likely treatment in parentheses:

SR-W001, Rad-Contaminated Solvents (thermal treatment)

SR-W003, Solvent Contaminated Debris (LLW) (macroencapsulation or stabilization)

SR-W012, Toxic Characteristic (TC) Solids (macroencapsulation or stabilization) 
SR-W018, Listed Incinerable Solids (macroencapsulation or stabilization)

SR-W035, Mixed Waste Oil-Sitewide (thermal treatment(aqueous portion stabilized))

SR-W042, Paints and Thinners (thermal treatment or stabilization)

SR-W051, Spent Filter Cartridges and Carbon Filter Media (macroencapsulation or stabilization)

SR-W055, Job Control Waste Containing Solvent Contaminated Wipes (macroencapsulation or stabilization)

SR-W070, Mixed Waste from Laboratory Samples (thermal treatment or stabilization)

SR-W071, Wastewater Suitable for Thermal Treatment or Stabilization

SR-W080, Wastes from Charleston Naval Shipyard (thermal treatment, macroencapsulation, or stabilization)

SR-W081, Reactive Ignitable Wastes (thermal treatment and/or stabilization)

SR-W087, Wastes from Bettis Atomic Power Laboratory (thermal treatment and/or stabilization)

\section{Estimated Schedule for Treatment of these Waste Streams}

Complete the Treatment:

\section{$\underline{\text { Schedule Assumptions }}$}

The ability to perform the treatment process depends on, but is not limited to, the following:

- The term "Non-PUREX" is a group of incinerable wastes previously destined to be treated in CIF.

- "Back-logged waste" is defined as incinerable mixed wastes which had been received into storage at RCRA-permitted or RCRA interim status storage facilities as of September 30, 1997.

- Wastes received into RCRA-permitted or RCRA interim status storage facilities between September 30, 1997, and September 30, 2000, are included in the above processing schedule. Wastes generated after September 30, 2000, are considered to be stored in accordance with the LDR storage requirements of R.61-79.268.50, including those wastes stored beyond one year to accommodate the vendor treatment schedule.

- Non-PUREX schedule is based on completion of characterization by FY06 and treatment in FY07 if commercial treatment capacity is available.

- Receipt by DOE-SR of adequate funding.

- The requirements for waste characterization, sorting, repackaging, and blending have been considered in determining this schedule.

- Receipt by DOE-SR of adequate funding identified for this project to support the schedule.

- Completion of appropriate NEPA documentation.

- Agreement by the regulatory agencies of South Carolina and contracting state at least 60 days prior to the planned shipment date of the waste.

- $\quad$ SRS shipment of the waste streams according to the shipment schedule.

- Waste treatment residues will be shipped by the offsite treatment contractor to a commercial disposal facility in conformance with LDR requirements, or will be returned to SRS.

\subsubsection{Commercial Vendor Deactivation Treatment}

Deactivation at a commercial vendor is the preferred option for the following waste streams (SR-W034, Calcium Metal.) 


\section{Estimated Schedule for Treatment of this Waste Stream}

Enter into contracts:

Submit waste processing schedule:
Procurement to establish work agreement with Commercial Vendor by 2QFY04 (completed 2/2/04). SRS to provide waste characterization data by 4QFY04 (completed 1/27/04).

SRS will submit a shipping schedule to SCDHEC by 2QFY05 (shipment completed 6/22/04).

\subsubsection{Preferred Treatment to be Determined}

The following stream is awaiting preferred treatment determination:

- SR-W091, Contaminated Debris (High Rad)

\subsection{Mixed Low-Level Waste Streams Requiring Technology Development}

Due to the complexity of some waste streams, technologies have not been developed that can adequately treat

the waste. Therefore treatment strategies for some waste streams have not been selected. This section addresses those mixed low-level waste streams that require technology development for the treatment of the waste stream.

\subsubsection{Development of Mobile Unit Technology}

Currently, there are no waste streams in this category.

\subsubsection{Development of Characterization Technology}

The waste stream, SR-W056, Job Control Waste with Enriched Uranium and Solvent Contaminated Wipes, previously included in this section, has been determined to be nonhazardous. Currently, there are no additional waste streams requiring development of chemical characterization technology. 


\subsubsection{Development of Treatment Technology}

The Waste stream SR-W045, PUREX waste was previously planned to be treated by thermal treatment at the Consolidated Incineration Facility (CIF). The preferred option for this waste stream is now to develop a direct solidification process using new technology which will allow the treatment of this waste stream to be completed on a greatly accelerated timescale and for a considerable cost-savings over the use of CIF. The selected treatment reagents added to solidify W045 will produce physical and chemical changes to immobilize the hazardous constituents of concern in the waste stream. The amount of reagents added will be part of the normal process of treating the waste to effectively immobilize the hazardous constituents. In the preliminary development work performed as part of the PUREX alternative treatment study, several commercial reagents were tested using waste concentrations of 50 weight percent and higher in the final wasteforms. The leachability of hazardous constituents in the final wasteforms was measured using the TCLP leach test. The hazardous constituent concentrations in the leachates were less than the LDR treatment standards, and also lower than the total concentrations in the final wasteforms. Since the leachate concentrations were lower than the wasteform concentrations, the stabilization reagents are immobilizing the hazardous constituents and reducing their mobility in the environment.

Further development is continuing to develop the specific treatment reagents and solidification process.

Estimated Schedule for Treatment of this Waste Stream

Complete treatment of SR-W045

Treatment to be completed by 4QFY07.

Schedule Assumptions

The ability to perform in accordance with this schedule for treatment of SR-W045 depends on, but is not

limited to, the following:

"Treatment" is defined as treatment of the waste to meet LDR standards in effect as of September 30, 2003.

Receipt by DOE-SR of adequate funding.

SCDHEC permit modifications are approved to support operation of a new organic solidification facility, or vendors are located that can receive and treat the waste.

Ongoing technology development process proceeds without the identification of major technical, regulatory, public acceptance or operational constraints which cause the selected stabilization process to be unsuitable for treating this waste stream.

\subsection{Mixed Low-Level Waste Streams for Which Further Characterization is Required}

This section discusses the schedules for those specific waste streams that require further chemical or radiological characterization prior to the selection of a preferred treatment option. The categories in this section are divided into waste streams to be further characterized and hazardous wastes awaiting radiological screening.

\subsubsection{Waste Streams to be Further Characterized}

Currently, there are no waste streams in this category.

\subsubsection{Hazardous Waste Awaiting Radiological Screening}

The following waste stream awaits radiological characterization/method development: 
SR-W078, LDR Hazardous Waste Awaiting Radiological Screening (new waste stream identified January 22, 1996; processing schedule submitted to SCDHEC January 9, 1997).

\section{Estimated Schedule for Treatment of this Waste Stream}

Complete radionuclide characterization for stream SR-W078

Submit information about wastes transferred from SR-W078 to an existing mixed waste stream:
Completed. Radiological characterization for this waste was completed 7/8/02. (Required 9/30/02)

Completed. Reports were submitted on a 6 month frequency to SCDHEC that identified waste quantities to be treated with an existing treatment path.

\subsection{Mixed Low-Level Waste Streams Requiring Radionuclide Decay Prior to LDR Treatment}

The preferred treatment option is treatment by aging in a regulated storage facility followed by combustion and/or appropriate mercury treatment.

SR-W036, Tritiated Oil with Mercury

SR-W090, Elemental Mercury - High Rad

\section{Estimated Schedule for Treatment of this Waste Stream}

The tritiated oil will be stored in a RCRA interim status, permitted, or accumulation area in compliance with

S.C. 61-79.262.34. Based on tritium half-life of 12 years and the present tritium contamination of up to 185 $\mathrm{Ci} / 1$, the projected worst-case radioactive decay time appropriate to eliminate release of excessive tritium during combustion would be 2060. A location for combustion and/or mercury treatment will be selected at a later date. See Volume II, Section 3.4.1, for additional details about this waste stream and its proposed treatment.

\subsection{Mixed Low-Level Waste Streams-Other Commitments}

\section{Aisle Spacing Requirements}

Commitment to Aisle spacing of Buildings 643-29E and 643-43E.
Completed. The storage configuration for Buildings $643-29 \mathrm{E}$ and $643-43 \mathrm{E}$ was revised to rows of two drums wide on 4/30/02 (required date 9/30/02). 


\section{Chapter 4. Mixed TRU (MTRU) Waste Streams}

The current DOE strategy for management of mixed transuranic (MTRU) waste is to maintain the MTRU wastes in safe interim storage; to characterize, certify, process if necessary, and package the wastes to meet the Waste Acceptance Criteria (WAC) of the Waste Isolation Pilot Plant (WIPP); and to permanently dispose of applicable MTRU waste in WIPP. The Defense Authorization Bill for federal FY 97, which contained amendments to the 1992 WIPP Land Withdrawal Act, was signed by the President on September 22, 1996, and the Secretary of Energy declared WIPP open and ready to receive waste in May 1998.

The amendments also exempt DOE from the requirements to obtain a RCRA no-migration determination from EPA. EPA agrees that the no-migration determination is redundant to the more stringent radioactive waste disposal standard, and that the exemption will not jeopardize the environment. WIPP has obtained a RCRA Part B Permit from the state of New Mexico to receive MTRU for disposal.

Site specific information is included in the following section to outline activities being performed at the Savannah River Site to maintain safe compliant storage, waste characterization activities, and other activities planned to support the ultimate goal of shipment to and disposal at WIPP.

The following project activity schedules are planned for the treatment of Mixed Transuranic (MTRU) waste in accordance with Section 2.2 of this volume. Refer to Table 1.1, Chapter 1 of this volume for the User's Guide to the waste streams. The User's Guide identifies each waste stream, the preferred treatment option (PO), and the location where the waste stream is discussed in Volumes I and II of the STP. The sections of this chapter are divided into treatment categories, as follows:

\subsection{Site MTRU Waste Management Approach}

\subsection{Site MTRU Waste Management Approach}

TRU waste is defined in DOE Order 435.1 as waste contaminated with alpha-emitting transuranic radionuclides (radionuclides with atomic numbers greater than 92) with half lives greater than twenty (20) years in concentrations greater than 100 nanocuries per gram $(\mathrm{nCi} / \mathrm{g})$ of waste matrix. TRU waste at the Savannah River Site that also contains hazardous constituents as defined in 40 CFR 261 and the South Carolina Hazardous Waste Management Regulations (SCHWMR) R.61-79.261 is managed in accordance with both DOE Orders and S.C. Hazardous Waste Management Regulations and is referred to as Mixed-TRU (MTRU) waste.

MTRU waste streams are, and have been, generated primarily by Plutonium Separations Facilities and the Analytical Laboratories. Other past generators of significantly smaller volumes include Naval Fuels Facility, the Reactor Facility, the Fuel Fabrication Facility, the High-Level Waste Tank Farms, and the Solid Waste Management Facility. In the 1970's, SRS received a large volume of MTRU waste from offsite generators including the Los Alamos National Laboratory, Knolls Atomic Power Laboratory, and the DOE Mound Site. DOE Mound Site MTRU waste shipments have been received (see Vol. II, Sec. 4.2.2).

MTRU waste generated at SRS is primarily job control waste which includes combinations of the following: plastic, paper, rubber, glassware, metal items, lead lined gloves, filters, used equipment and other contaminated materials from routine processing.

Due to the variety of container types, MTRU waste is generally categorized by its container: drums, polyethylene boxes, concrete casks, large steel black boxes, and other odd-sized containers. The type container also dictates the storage configuration, for example drums are either stored in culverts which are stored on uncovered pads that are exposed to the weather or stored directly on covered pads. 
Currently, five waste streams are managed as MTRU waste.

Waste Stream No.

SR-W006

SR-W026

SR-W027

SR-W053

SR-W089
Description

CH Mixed TRU/Liquids

CH Mixed TRU/Thirds

CH Mixed TRU/F-Listed Solvents

Rocky Flats Ash

MTRU Waste Received From Mound Site

\section{TRU Pad Storage Reconfiguration}

Due to a change in the interpretation of the aisle spacing guidelines, SRS was required to revise the storage configuration to 2-drum wide rows on covered TRU pads. The covered TRU pad storage drum reconfiguration was complete by 4/30/02 (required date 5/31/02). An exception to this requirement is the storage of TRUPACT II payloads on TRU Pad 3 and in building 724-8E. A "payload" is a configuration of 14 TRU drums that will be placed in the TRUPACT II shipping container for shipment to WIPP. The following conditions apply to storage of TRUPACT II payloads:

1) No more than 21 payloads may be stored at any one time.

2) Payloads may be stored only on TRU Pad 3 and in the 724-8E Facility.

3) Storage is limited to a maximum of 4 months for any one payload. Each payload must be clearly labeled to show the date when it was configured.

4) Payloads may be staged only 1 payload high (SRS will need to submit seismic analysis prior to approval to stack 2 high in racks).

A progress report describing the status of the SRS TRU storage program will be submitted to SCDHEC every 5 years, commencing June 30, 2007. At this time the continued need for the exception to SCHWMR R.6179.265.35 for the TRUPACT payloads will be evaluated.

\section{TRU Pad Capacity Reduction}

Beginning in FY03, TRU Pad RCRA Interim Status capacity will be reduced by 422,000 gallons per year until capacity is reduced by 2.7 million gallons. TRU Pad storage capacity on October 1, 2004 was 3,195,580 gallons.

\section{Closure Plan Submittal}

Completed: The 724-8E Closure Plan was submitted to DHEC 10/29/03.

\section{$\underline{\text { Schedule Assumptions }}$}

The ability to perform in accordance with the estimated schedule depends on, but is not limited to, the following:

- $\quad$ The STP Cleanup Credits agreement is in effect.

- Receipt by DOE-SR of adequate funding specifically identified for this project to support the schedule, 
- Resolution of any technically related finding(s) that might result from an operational readiness selfassessment or the systems testing phase,

- No changes in regulations, statutes, or the regulator's interpretations,

- Operation of WIPP until 2034 as described in the Carlsbad Field Office "National TRU Waste Management Plan" (Document DOE/MP-96-1204, Revision 3),

- Schedule can be extended where good cause exists, including, but not limited to:

- circumstances unforeseen at the time the schedule was prepared that significantly affect the work required,

- delays in review of permit application(s), permit(s), or delays in approval of any other documents or other items needed to satisfy the requirements outlined,

- any other event or series of events including, but not limited to, the discovery of new technological information or technological barriers that significantly affects the work required, or

- a delay caused by insufficient funding where DOE, in a timely manner and in good faith, requested adequate funding in accordance with the federal appropriations process, but Congress failed to appropriate such funding.

- Receipt of newly generated waste at the TRU pads is in the same proportions as historically received. (This includes the ratio of mixed to non-mixed TRU waste received, as well as the distribution between pad stored and culvert stored TRU waste drums. The storage configuration needed for each container of newly generated waste will impact the storage area remaining for drum storage on these pads). 


\section{Chapter 5. High-Level Radioactive Waste (HLW)}

The following project activity schedules are planned for the treatment of HLW in accordance with Section 2.2 of this volume. Refer to Table 1.1, Chapter 1 of this volume for the User's Guide to the waste streams. The User's Guide identifies each waste stream, the preferred treatment option (PO), and the location where the waste stream is discussed in Volumes I and II of the STP.

\section{High-Level Waste Treated Onsite in Existing Facilities}

Currently, DWPF is the only facility at SRS that treats high-level waste.

Vitrification in the DWPF is the preferred treatment option for certain mixed waste streams, including, but not limited to, the following:

SR-W016， 221-F Canyon High-Level Liquid Waste

SR-W017， 221-H Canyon High-Level Liquid Waste

\section{Estimated Schedule for this Onsite Facility}

Submittal of all applicable permit applications:

Entering into contracts:

Initiating construction:

Conducting systems testing:

Commencing operations:

Submit processing backlogged and currently generated mixed waste schedule:
Completed. The industrial wastewater treatment construction permit was received in November 1990. The operating permit was received in August 1992. The NESHAP permit was granted in April 1988. The air emissions construction permit was granted in July 1984.

Completed. Contracts were entered prior to approval of the STP on September 29, 1995.

Completed. DWPF construction was initiated in April 1983.

Completed. Systems testing was initiated in January 1993.

Completed. Operations commenced March 7, 1996.

Completed. The schedule was submitted May 21, 1996. Commitments in the schedule stated that DWPF would remain in a start-up mode through 1996. During that time operating conditions will be confirmed. Upon the beginning of full operations, DWPF will maintain canister production sufficient to meet the commitment for the removal of the backlogged and currently generated waste inventory by 2028 . 


\section{APPENDIX A}

\section{CURRENT FISCAL YEAR COMMITMENTS FEDERAL FISCAL YEAR 2004}

Appendix A is a summary of commitments compiled from Volume I for the current federal fiscal year 2004, including the deliverable date to meet each commitment. The process used to prepare this Appendix is found in Section 2, Chapter 2, of this volume. 


\section{Appendix A}

\section{Project Activities Schedule for the Current Federal Fiscal Year 2005}

Federal Fiscal Year Identified: 2005

\begin{tabular}{|c|l|c|}
\hline \multicolumn{2}{|c|}{ No. } & \multicolumn{1}{|c|}{ Commitment Schedule } \\
\hline A-1 & $\begin{array}{l}\text { Submit treatment strategy for stream SR-W090, Elemental Mercury - } \\
\text { High Rad.. }\end{array}$ & $\begin{array}{c}\text { Completed. } \\
\text { (Date due } \\
10 / 14 / 04)\end{array}$ \\
\hline A-2 & $\begin{array}{l}\text { Submit shipping schedule for SR-W048, SR-W073, SR-W082, and } \\
\text { SR-W088 within 90 days of vendor approval of the waste. }\end{array}$ & $11 / 23 / 04$ \\
\hline A-3 & $\begin{array}{l}\text { Submit shipping schedule for remaining SR-W069 lead within 24 } \\
\text { months of contract execution. }\end{array}$ & $12 / 10 / 04$ \\
\hline A-4 & Submit shipping schedule for stream SR-W034, Calcium Metal. & $\begin{array}{c}\text { Completed. } \\
\text { (Date due } \\
03 / 31 / 05)\end{array}$ \\
\hline A-5 & $\begin{array}{l}\text { Submit shipping schedule for stream SR-W091, Contaminated } \\
\text { Debris (High Rad). }\end{array}$ & $08 / 30 / 05$ \\
\hline
\end{tabular}




\section{APPENDIX B COMMITMENTS FOR UPCOMING FEDERAL FISCAL YEAR +1 AND +2}

Appendix B is a summary list of commitments compiled from Volume I for the first and second years after the current federal fiscal year including the deliverable dates for each commitment. The process used to prepare this Appendix is found in Section 2, Chapter 2, of this volume. 


\section{Appendix B}

\section{Project Activities Schedule for the Federal Fiscal Year +1 and +2}

Federal Fiscal Year Identified: 2006 and 2007

\begin{tabular}{|c|l|c|}
\hline \multicolumn{1}{|c|}{ Commitment Schedule } & Date \\
\hline B-1 & Complete the treatment of backlogged Non-PUREX Waste. & $06 / 30 / 07$ \\
\hline B-2 & Complete treatment of SR-W045, PUREX Waste. & $09 / 30 / 07$ \\
\hline
\end{tabular}




\section{Volume II}

\section{Volume II - Background Volume}

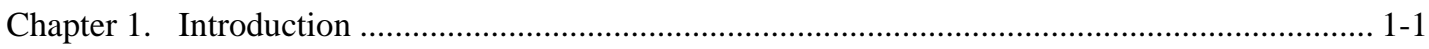

1.1 Regulatory Basis and STP Development ......................................................... 1-1

1.2 Documents and Activities Related to STP Development ...................................... 1-2

1.3 Site History and Mission................................................................................ 1-5

1.4 STP Organization.......................................................................................... 1-10

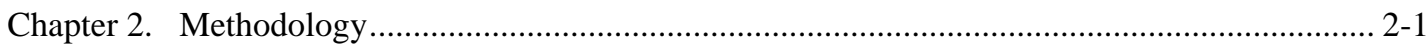

2.1 Assumptions Used for Preparation of Initial STPs ........................................... 2-1

2.2 Treatment Options Selection Process ……………............................................ 2-2

2.3 Coordination with Regulatory Agencies and Other Stakeholders ......................... 2-12

2.4 Mixed Waste Characterization............................................................................... 2-14

2.5 Waste Minimization/Pollution Prevention (WMin/P2) ........................................ 2-18

Chapter 3. Mixed Low-Level Waste Streams ............................................................................. 3-1

3.1 Mixed Low-Level Waste Streams with Treatment Capacity ................................. 3-1

3.2 Mixed Low-Level Waste Streams Requiring Technology Development ............... 3-37

3.3 Mixed Low-Level Waste Streams for Which Further Characterization

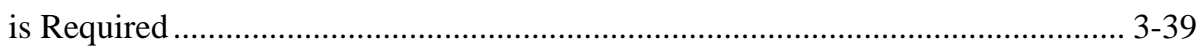

3.4 Mixed Low-Level Waste Streams Requiring Radionuclide Decay Prior to LDR Treatment ................................................................................................. 3-39

3.5 SRS Treatment Facilities that have Discontinued/Suspended Operations............. 3-41

Chapter 4. Mixed Transuranic Waste (MTRU).............................................................................. 4-1

4.1 Site MTRU Waste Management Approach …………........................................... 4-1

4.2 MTRU Waste Streams Proposed for Shipment To WIPP ..................................... 4-3

Chapter 5 High-Level Radioactive Waste (HLW) ....................................................................... 5-1

Chapter 6. Future Generation of Mixed Waste Streams................................................................. 6-1

6.1 Soil and Groundwater Closure Waste.................................................................. 6-1

6.2 Decontamination and Demolition (D\&D) Waste................................................... 6-1

6.3 Additional Waste Streams ................................................................................ 6-2

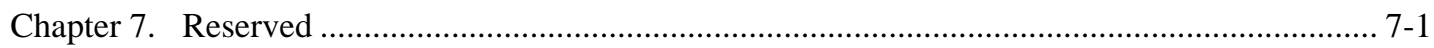

Chapter 8. Disposal in Support of the STP Discussions .............................................................. 8-1

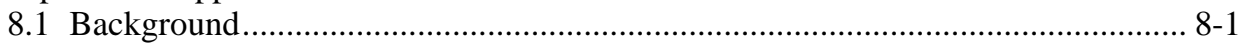

8.2 Current Status of Disposal Sites .................................................................. 8-1

Chapter 9. Treatment Facilities and Treatment Technologies ........................................................ 9-1

9.1 SRS Facility Descriptions ............................................................................. 9-1

9.2 Process Descriptions ............................................................................................. 9-3

9.3 Planned/Proposed Facilities or Technologies …………….................................. 9-6

Chapter 10. Offsite Waste Streams for which SRS is the PREFERRED OPTION ............................ 10-1

Chapter 11. Volume Summary Information ............................................................................ 11-1

Chapter 12. Acronyms and Definitions Glossary …………………............................................ 12-1 


\section{List of Tables}

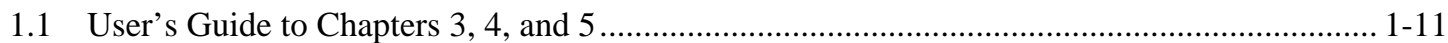

1.2 EPA Hazardous Waste Codes with Subcategories ........................................................... 1-27

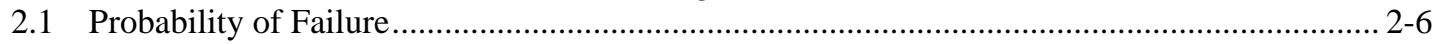

2.2 Consequences of Failure .............................................................................................. 2-6

2.3 Attributes and Enabling Statements for Options Analysis .................................................... 2-10

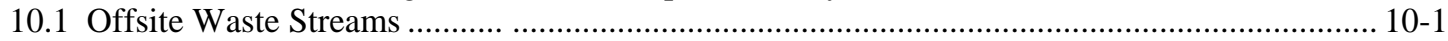

11.1 Volume Summary by Waste Stream Number...................................................................... 11-1

11.2 Volume Summary by Treatment Facility .......................................................................... 11-7

11.3 Mixed Waste Treatment Residue Summary ......................................................................... 11-14

\section{List of Figures}

1.1 General Location of Savannah River Site ........................................................................ 1-8

1.2 Location of Major Production, Support, and Research and Development

Areas at the Savannah River Site.

\section{List of Waste Streams}

SR-W001 Rad-Contaminated Solvents .............................................................................. 3-16

SR-W002 Rad-Contaminated Chlorofluorocarbons ……......................................................... 1-11

SR-W003 Solvent Contaminated Debris (LLW) _.............................................................. 3-18

SR-W004 M-Area Plating Line Sludge from Supernate Treatment ……...................................... 1-11

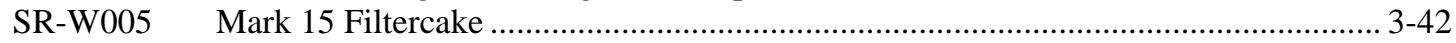

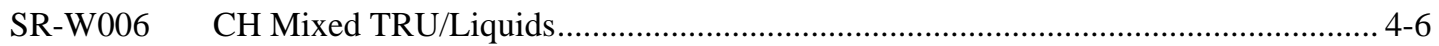

SR-W007 SRNL Sample Material …………………...................................................... 1-11

SR-W008 Separations Area Sample Receipts from SRNL ........................................................ 3-43

SR-W009 Silver Coated Packing Material ……….............................................................. 3-3

SR-W010 Scintillation Solution

SR-W011 Cadmium Coated HEPA Filters ................................................................... 3-44

SR-W012 Toxic Characteristic Solids .................................................................................. 3-21

SR-W013 Low-Level Waste (LLW) Lead -To be Decontaminated............................................... 3-43

SR-W014 Elemental (Liquid) Mercury - Sitewide .......................................................... 3-10

SR-W015 Mercury/Tritium Contaminated Equipment ……..................................................... 3-44

SR-W016 221-F Canyon High-Level Liquid Waste ................................................................... 5-1

SR-W017 221-H Canyon High-Level Liquid Waste ................................................................. 5-2

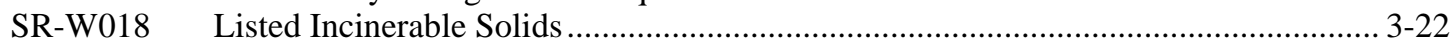

SR-W019 244-H RBOF High Activity Liquid Waste.................................................................. 1-12

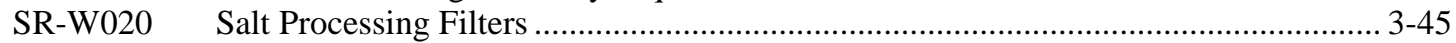

SR-W021 Poisoned Catalyst Material.............................................................................. 1-12

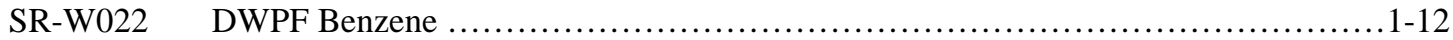

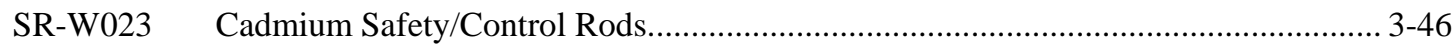

SR-W024 Mercury/Tritium Gold Traps ……….............................................................. 3-46

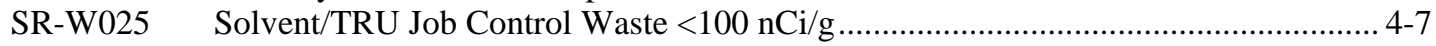

SR-W026 CH Mixed TRU/Thirds ………...................................................................... 4-4

SR-W027 CH Mixed TRU/F-Listed Solvents........................................................................ 4-5

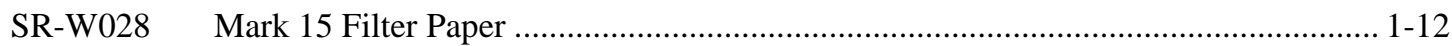

SR-W029 M-Area Sludge Treatability Samples ................................................................... 3-42

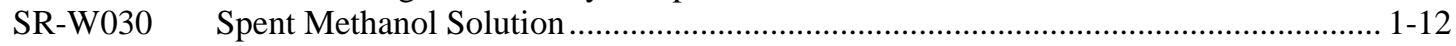

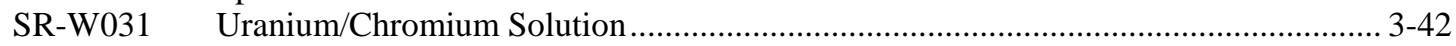

SR-W032A Mercury-Contaminated Heavy Water ..................................................................... 3-42

SR-W032B Mercury-Contaminated Heavy Water Residues .............................................................. 3-47

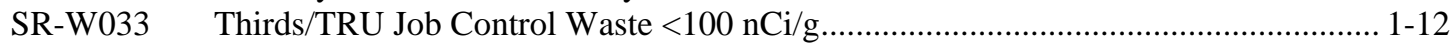

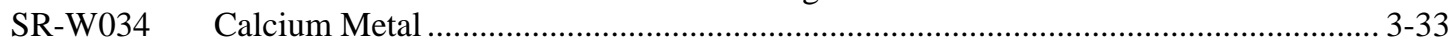

SR-W035 Mixed Waste Oil - Sitewide …………........................................................... 3-23 
SR-W036

SR-W037

SR-W038

SR-W039

SR-W040

SR-W041

SR-W042

SR-W043

SR-W044

SR-W045

SR-W046

SR-W047

SR-W048

SR-W049

SR-W050

SR-W051

SR-W052

SR-W053

SR-W054

SR-W055

SR-W056

SR-W057

SR-W058

SR-W059

SR-W060

SR-W061

SR-W062

SR-W063

SR-W064

SR-W065

SR-W066

SR-W067

SR-W068

SR-W069

SR-W070

SR-W071

SR-W072

SR-W073

SR-W077

SR-W078

SR-W079

SR-W080

SR-W081

SR-W082

SR-W083

SR-W084

SR-W085

SR-W086

SR-W087

SR-W088

SR-W089

SR-W090

SR-W091
Tritiated Oil with Mercury ................................................................................ 3-39

M-Area Plating Line Sludges ............................................................................ 3-42

Plating Line Sump Material ..................................................................... 3-42

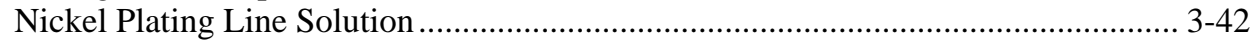

Listed Stabilized Sludge/Listed LDR-Compliant Debris ...................................... 3-48

Aqueous Mercury and Lead ................................................................................. 3-49

Paints and Thinners . ...................................................................................... 3-24

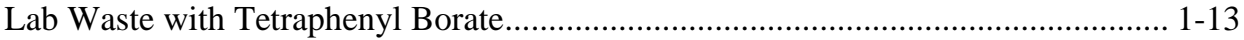

Tri-Butyl-Phosphate \& n-Paraffin-TRU ........................................................ 1-13

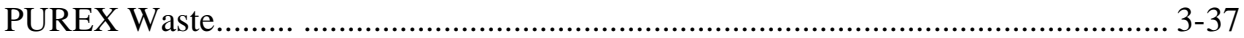

Consolidated Incineration Facility Ash ............................................................... 3-50

Consolidated Incineration Facility Blowdown …................................................ 3-50

Soils from Spill Remediation ................................................................... 3-12

Tank E-3-1 Clean Out Material............................................................................... 1-13

Waste to Support High-Level Waste (HLW) Processing Demonstrations ................ 3-55

Spent Filter Cartridges and Carbon Filter Media ..................................................... 3-25

Cadmium-Contaminated Glovebox Section...................................................... 1-14

Rocky Flats Ash ............................................................................................. 4-7

Enriched Uranium Contaminated with Lead ................................................. 1-14

Job Control Waste Containing Solvent Contaminated Wipes................................. 3-26

Job Control Waste with Enriched Uranium and Solvent Contaminated Wipes .......... 1-14

D-Tested Neutron Generators ......................................................................... 1-14

Mixed Sludge Waste with Mercury from DWPF Treatability Studies ..................... 3-56

Tetrabutyl Titanate (TBT) ...................................................................... 1-14

Tritiated Water with Mercury ...................................................................... 3-4

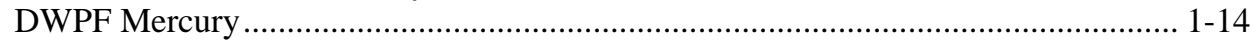

Low-Level Contaminated Debris .......................................................................... 3-6

Macroencapsulated Low-Level Waste (TC) ....................................................... 3-56

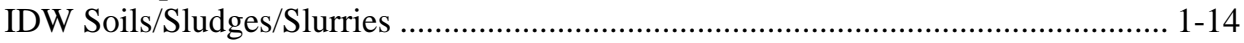

IDW Monitoring Well Purge/Development Water ........................................ 1-14

IDW Debris .................................................................................................. 1-14

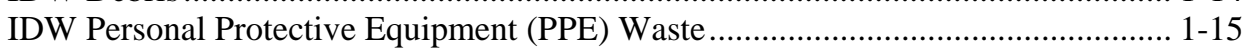

Elemental (Liquid) Mercury-Sitewide.................................................................. 3-10

Low-Level Waste (LLW) Lead - To be Macroencapsulated Offsite........................ 3-9

Mixed Waste from Laboratory Samples ....................................................... 3-27

Wastewater Suitable for Thermal Treatment or Stabilization ................................... 3-30

Supernate or Sludge Contaminated Debris from High-Level

Waste (HLW) Operations ................................................................................. 3-57

Cadmium-Containing Raschig Rings .............................................................. 3-13

Aqueous Characteristic Wastewater............................................................ 3-1

LDR Hazardous Waste Awaiting Radiological Screening ................................... 3-58

Polychlorinated Biphenyl (PCB) Mixed Waste ...................................................... 3-5

Charleston Naval Shipyard Waste (CN-W001, CN-W004) ....................................... 3-31

Reactive/Ignitable Waste...................................................................................... 3-31

Radioactive Chemicals, Metals, and Pesticides (CMP) Soil .................................. 3-14

Mercury- and Chromium-Contaminated Residues............................................. 1-16

Remediation Waste Soils that Meet LDR ….................................................... 1-16

Rocky Flats Plutonium Fluoride Residues ......................................................... 1-16

Characteristically Hazardous Non-Incinerable Solids ............................................ 3-11

Bettis Atomic Power Laboratory Contaminated Oil .............................................. 3-32

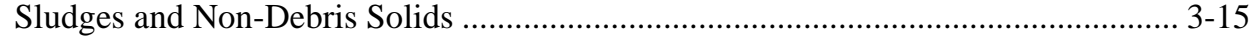

TRU Mixed Waste from Mound Site ..................................................................... 4-8

Elemental Mercury - High Rad................................................................... $3-40$

Contaminated Debris (High Rad) ....................................... 33 


\section{Chapter 1. Introduction}

This chapter discusses the development of the Site Treatment Plan (STP) as well as the regulatory basis behind the STP. It also covers the history and mission of the Savannah River Site (SRS). In Section 1.4, the organization of the STP is described, and several tables are included for reference.

\subsection{Regulatory Basis and STP Development}

The Resource Conservation and Recovery Act (RCRA) Land Disposal Restrictions (LDR) require the treatment of hazardous waste (including the hazardous component of mixed waste) to certain standards before the waste can be land disposed and prohibit storage of hazardous wastes that do not meet LDR standards, except for the purposes of accumulating sufficient quantities to facilitate proper recovery, treatment, or disposal of the waste. U.S. Department of Energy (DOE) is currently storing mixed waste inconsistent with the LDR provisions because the treatment capacity for such wastes, either at DOE sites or in the commercial sector, is not adequate or is unavailable at this time.

The Federal Facility Compliance Act (FFCAct), signed on October 6, 1992, waived sovereign immunity for fines and penalties for RCRA violations at federal facilities. However, the FFCAct postponed the waiver for 3 years for LDR storage prohibition violations for DOE's mixed wastes and required DOE to prepare plans for developing the required treatment capacity for its mixed waste at each site at which it stores or generates mixed waste. Each plan may be approved, approved with modification, or disapproved by the state after consultation with other affected states and consideration of public comment. Upon approval of the plan, the state shall issue an order requiring compliance with the approved plan. The FFCAct further provides that DOE will not be subject to fines and penalties for LDR storage prohibition violations for mixed waste as long as it is in compliance with an approved plan and order.

The FFCAct requires the plans to contain schedules for developing capacity for mixed waste for which identified treatment technologies exist and for mixed waste without an identified existing treatment technology, schedules for identifying and developing technologies. The FFCAct also requires the plan to provide certain information where radionuclide separation is proposed. The FFCAct states that the plans may provide for centralized, regional, or onsite treatment of mixed waste, or any combination thereof, and requires the states to consider the need for regional treatment facilities in reviewing the plans.

The Department of Energy (DOE) was required by Section 3021(b) of RCRA, as amended by the FFCAct, to prepare site treatment plans describing the development of treatment capacities and technologies for treating mixed waste. Plans were required for facilities at which DOE generates or stores mixed waste, defined by the FFCAct as waste containing both a hazardous waste subject to RCRA, and a source, special nuclear, or byproduct material subject to the Atomic Energy Act (AEA) of 1954 (42 U.S.C. 2011 et seq.). The Savannah River Site (SRS) Site Treatment Plan was provided to South Carolina, approved, and incorporated into a Consent Order, effective September 29, 1995, in accordance with the FFCAct. Provision 3 under Conclusions of Law in the STP Consent Order requires that updates to the STP be submitted annually no later than April 30 of each year.

DOE and SRS followed an iterative process in developing the plans, as described in an April 6, 1993, Federal Register (FR) notice (58 FR 17875), working closely with state regulatory agencies and the Environmental Protection Agency (EPA) at the site and national level throughout the process. The STP development followed a three-step interim process - a Conceptual Site Treatment Plan (CSTP) submitted in October 1993, a Draft STP (DSTP) submitted in August 1994, and a Proposed STP (PSTP) submitted in March 1995, which were provided to regulatory agencies and made publicly available. The CSTP identified a range of preliminary options for treating the mixed waste at SRS. The DSTP identified site-specific preferred treatment options that had not yet been evaluated for impacts to other DOE sites or to the overall DOE program. The PSTP further narrowed the preferred treatment options based upon feedback from the State of South Carolina and the public. DOE initially planned to submit the PSTP at the end of February 1995. However, DOE revised its submittal date with the support of the states and EPA to allow for additional discussions (see 60 FR 10840, February 28, 
1995). The PSTP was submitted to South Carolina Department of Health and Environmental Control (SCDHEC) on March 30, 1995.

The PSTP was modified in response to comments from the South Carolina Department of Health and Environmental Control (SCDHEC) and the public. On September 20, 1995, the PSTP was approved by SCDHEC subject to subsequent specific modifications. The approved STP and other related information are available at the public reading room at the University of South Carolina-Aiken library.

This approved STP, now referred to simply as the STP, contained DOE's preferred options developed after evaluation and integration of the site-specific treatment options contained in the DSTP and the PSTP of the other sites with DOE mixed waste. The process DOE followed was coordinated with state and EPA regulators and is described in Section 2.2 of this volume. DOE believes the treatment options contained in the STP represent a sensible national configuration for mixed waste treatment systems that balances DOE's interests and concerns and the input DOE received on the PSTP from the regulatory agencies and others. As new information is learned affecting the status of mixed waste inventories or treatment capacity, modifications are proposed in annual updates to the STP.

The approved STP also contains schedules for constructing new facilities, modifying existing facilities, and otherwise obtaining treatment for mixed wastes. DOE faces increasingly tight budgets throughout the DOE complex and anticipates that funding will continue to be constrained. The schedules in the STP and annual updates reflect those constraints and modifications that may be necessary in treatment options or schedules in response to constraints. DOE has provided schedules in the STP and annual update to support further discussions with the expectation that schedules will require some modifications as mixed waste treatment efforts progress.

The schedules contained in the STP and annual update are based on funds currently budgeted for and projected to be available for waste management activities. As a result, schedules in the STP and annual update for some facilities, particularly the largest and most costly facilities, may be protracted.

DOE has discussed with states and EPA the difficulty DOE faces in providing timely schedules for some new treatment facilities given current budgetary constraints and the need to consider whether funds from other activities should be shifted to support more timely schedules. The states and EPA recommended that the STP be submitted with schedules consistent with current budget and priorities, even though they recognized schedules may be extended. This process has continued through the submittal of the annual update. As part of its efforts to develop budget requests, DOE has asked regulatory agencies to work with DOE and other interested parties at the site and national level to assist DOE in prioritizing its activities, including mixed waste treatment, and assessing activities under way and that need to be accomplished at the Site.

DOE anticipates that modifications and adjustment to the STP in annual updates will be necessary because of the technical and funding uncertainties that naturally exist with long-term activities like those covered by the Plans. For example, emerging or new technologies not yet considered may be identified in the future that provide opportunities to manage waste more safely and effectively, and at a lower cost, than the current technologies identified in the Approved Plan. DOE will continue to evaluate and develop technologies that offer potential advantages in the areas of public acceptance, risk abatement, and performance and life-cycle cost. Should more promising technologies be identified, DOE may request a modification of its treatment plan through the annual update or other processes in accordance with provisions of the STP and/or the Consent Order.

\subsection{Documents and Activities Related to Site Treatment Plan Development}

\subsubsection{Inventory Determination}

Other DOE efforts are closely linked to the STP development. These include the Mixed Waste Inventory Report (MWIR), activities conducted pursuant to the National Environmental Policy Act (NEPA) and other 
planning and management actions, and compliance and cleanup agreements containing commitments relevant to treatment of mixed waste.

The MWIR initially required by the FFCAct, provided an inventory of mixed waste currently stored, generated, or expected to be generated over the next 5 years at each DOE site and treatment capacities and technologies. The Interim MWIR, published by DOE in April 1993, provided information on each mixed waste stream generated or stored by the DOE sites. DOE made updated waste stream and technology data available to the states and EPA. The 1995 MWIR, which was distributed to the states, represents the DOE's mixed waste inventory at SRS as of September 1994. To reflect the most current information in the STP Annual Update, SRS plans to update the mixed waste inventories each year.

The STP reflects the most current and accurate data on waste streams and technology needs. As a result, there may be some differences in the reported volumes and forecasts from the previous annual update of the STP. In general, these differences result from refinements of volume estimates for mixed waste generation as better information on stored waste or more accurate estimates of future waste generation have become available. Other differences involve mixed waste streams that have been combined, deleted, or have had waste stream volumes added. Some waste streams or volumes have been treated to LDR standards and no longer need to be addressed; however some waste streams, such as SR-W040, have been retained in the STP at this time for reference even though the mixed waste in this stream meets the LDR standards.

\subsubsection{NEPA Documents}

\section{The National Environmental Policy Act (NEPA)}

NEPA requires federal agencies to assess and address environmental impact of their proposed activities and consider alternative actions. NEPA requires detailed Environmental Impact Statements (EIS) for major federal projects. Environmental Assessments (EA) are prepared for smaller activities with unclear levels of impact to determine the need to prepare an EIS. Small, routine activities can be categorically excluded from NEPA review under the Council on Environmental Quality (CEQ) and DOE regulations. NEPA provides for public review of and input to federal actions. The status of SRS facilities under NEPA is indicated below.

While there is no sitewide EIS for SRS, the EIS for Waste Management Activities for Groundwater Protection at SRP (DOE/EIS-0120), prepared in 1987, addressed sitewide waste management issues. Existing, planned, and proposed mixed waste treatment facilities have been and are being addressed under NEPA. Summary information providing a NEPA status on mixed waste treatment facilities is found in succeeding paragraphs.

Defense Waste Processing Facility (DWPF): An EIS and Record of Decision (ROD) were published in 1982 documenting the decision of DOE to construct and operate DWPF. Since then, DOE has modified the DWPF process and facilities to improve efficiency and safety. A supplemental EIS (SEIS) was prepared to address these modifications.

This SEIS examined the environmental impacts of the modifications made to the DWPF and associated high-level waste facilities at SRS and enabled DOE to determine that the decisions reached as a result of the 1982 EIS and subsequent Supplement Analysis remain valid in light of process and facility modifications made over the last 12 years.

By way of history, the DWPF modifications addressed in the SEIS included the following: In-Tank Precipitation (ITP), Saltstone Processing and Disposal, the Late-Wash Facility addition, nitric acid introduction, ammonia mitigation modification, hydrogen modifications, and benzene treatment. The SEIS evaluated additional modifications that may result from the need to mitigate cumulative impacts or to further enhance safety and efficiency. A final SEIS was issued in November 1994. Following the public review of this document, a ROD was issued on March 28, 1995.

The SRS Salt Processing Alternative Final SEIS was issued June 2001, and the ROD was published October 17, 2001 (66 Fed. Reg. 52752). This ROD considered the alternatives for separating radioactive salt waste into high activity and low activity fractions. The technology chosen by DOE to perform this separation is the Caustic Side Solvent Extraction process. Among other reasons, DOE chose the Solvent Extraction technology 
on the basis of the simplicity with which the product stream could be incorporated into the current DWPF vitrification process.

Consolidated Incineration Facility (CIF): An EA was completed, and a Finding of No Significant Impact (FONSI) was issued by DOE-Headquarters (HQ) on December 18, 1992. The CIF is currently in suspension of operation.

M-Area Vendor Treatment Facility: An EA was prepared for this project. A FONSI was issued by DOEHQ on August 1, 1994. The M-Area Vendor Treatment Facility was closed per the Clean Water Act as of October 1, 1999.

\section{Waste Management Environmental Impact Statement (WMEIS)}

DOE-SR prepared a sitewide Waste Management EIS (WMEIS) to provide a basis to select a sitewide strategy to manage present and future SRS waste generated from ongoing operations, environmental restoration activities, and decontamination and decommissioning activities. In selecting a sitewide SRS waste management strategy, technology development and waste minimization were considered. In addition, the WMEIS provided a baseline for analyzing future waste management activities and evaluating specific waste management alternatives. DOE could, in turn, base supplemental EISs or EAs on the WMEIS to evaluate future mission activities, decontamination and decommissioning alternatives, and technological development opportunities. The WMEIS included the investigation of existing mixed waste treatment facilities such as the F-Area and HArea Effluent Treatment Facility (ETF), as well as facilities under construction or planned, including the CIF and the Transuranic Waste Certification/Characterization Facility (TWCCF). SRS reassessed the NEPA evaluations performed for these facilities to determine whether, in light of changing DOE goals and missions, the evaluations performed in regard to these projects remain appropriate. All No Action and Proposed Action alternatives regarding these facilities were evaluated in the WMEIS.

Analysis of options for onsite treatment of SRS mixed waste streams developed by the STP supported the WMEIS for mixed waste and was the foundation for EIS evaluations regarding mixed waste.

The final WMEIS was made available to the public in July 1995. A ROD was approved and issued on September 23, 1995. A second ROD was issued on May 9, 1997, covering additional mixed and transuranic (TRU) waste activities. A supplemental ROD was issued June 4, 2001 for the WMEIS that facilitates offsite treatment and disposal of mixed wastes.

\section{The Waste Management Programmatic Environmental Impact Statement (WMPEIS)}

DOE has prepared a Programmatic Environmental Impact Statement (PEIS) to formulate and implement a complexwide waste management program for five types of radioactive and hazardous waste, including mixed waste, in a safe and environmentally sound manner and in compliance with applicable laws, regulations, and standards. The PEIS presented to the public, states, EPA, and DOE understanding of impacts to human health and the environment together with the costs associated with a wide range of alternative strategies for managing DOE's environmental program. The PEIS examined the following waste types and activities: high-level, transuranic mixed low-level, low-level, and hazardous waste. The analysis for the waste management PEIS evaluated decentralized, regional, and centralized approaches for storage of high-level waste, treatment and storage of transuranic waste, treatment and disposal of low-level and mixed low-level waste, and treatment of hazardous waste.

Development of the Waste Management (WM) PEIS was coordinated with the preparation of STPs under the FFCAct. Information generated to support the WMPEIS (e.g., hypothetical configurations, preliminary risk analyses, and cost studies) was shared with states to support STP discussions.

The draft WMPEIS was presented for public comment in October 1995. The final PEIS was issued in May 1997, and ROD for treatment and disposal of Mixed Waste was issued February 2000. 


\subsubsection{Environmental Restoration/Waste Management Outyear Budget}

DOE's Office of Environmental Management (EM) uses a variety of interrelated planning initiatives to accomplish its mission. One of these is the Outyear Budget. The Outyear Budget is the principal planning document for EM activities and is updated annually. The Outyear Budget identifies activities needed to accomplish EM's mission over the planning period. The SRS portion of the Outyear Budget is available as a part of the supporting data and documentation prepared for the STP and can be reviewed by interested parties.

\subsubsection{Waste Management Plans}

To provide tools for planning consistent with the SRS Outyear Budget but with further, more specific detail on waste management activities, SRS has developed waste management plans. These plans have been organized according to the type of waste being discussed. The System Plan for the Solid Waste Division addresses planning for sanitary waste, hazardous waste, mixed low-level waste, low-level radioactive waste, and transuranic waste. The High-Level Waste System Plan addresses planning for the high-level wastes that are liquid radioactive wastes and include high-level mixed wastes.

The purpose of the System Plan for the Solid Waste Division is to present recommended options for managing solid waste at SRS. The plan identifies the approximate funding and schedule requirements and the numerous issues and assumptions that must be addressed during implementation. The System Plan for the Solid Waste Division has been developed to meet current and anticipated solid waste needs at SRS and provide a strategic plan for the treatment, storage, and disposal of SRS solid waste streams. It has been recognized that the strategy for mixed waste developed in the System Plan for the Solid Waste Division is dependent on the development of the SRS STP and input into the STP by the regulatory agencies and other stakeholders. As a result, significant changes could be made to the mixed waste management strategy in the System Plan for the Solid Waste Division. The plan will be revised on a regular basis to reflect changes as a result of the STP development as well as new regulatory developments, advances in technology, and funding changes.

The High-Level Waste System Plan provides the same long-range planning function for high-level waste as the System Plan for the Solid Waste Division provides for solid waste. Mixed high-level waste treatment also will be affected by developments in the STP, and the plan for high-level waste must reflect the changes brought about as the STP is prepared and approved.

\subsubsection{Compliance Agreements}

In addition to SCDHEC Consent Order 95-22, another agreement that concerns mixed waste activities has been executed among SRS, the EPA, and SCDHEC.

The Federal Facility Agreement (FFA): Section 120, Federal Facilities, of the Comprehensive Environmental Response, Compensation, and Liability Act (CERCLA), requires that a federal facility placed on the National Priorities List (NPL) enter into an interagency agreement (FFA) with the EPA for the expeditious completion of all necessary remedial actions at the facility.

SRS has entered into an FFA with EPA-IV and SCDHEC that directs the comprehensive remediation of SRS. It details the method by which the three parties will interact in the process of remediating SRS. It directs the three parties in their respective responsibilities, and requires the parties to meet, discuss, and prepare schedules for the remediation. The FFA contains requirements for the prevention and mitigation of releases or potential releases from the high-level radioactive waste tank systems. It also affects how environmental restoration activities at SRS dealing with mixed waste will be undertaken. See Chapter 6 regarding management of environmental restoration and decommissioning and decontamination wastes.

\subsection{Site History and Mission}

This section discusses the role of SRS, the principal operations located at the SRS site, and the principal mixed waste facilities at SRS. 


\subsubsection{Role of the Savannah River Site}

SRS was established by the United States Atomic Energy Commission (USAEC) in 1950 to produce and recover nuclear materials (primarily tritium, plutonium-239 $\left[\mathrm{Pu}^{239}\right]$, and highly enriched uranium fuel) for national defense and medical use. Most of the nuclear materials produced at SRS were used for the production of components for nuclear weapons necessary for the national defense in accordance with DOE authority and responsibility under the AEA. Figure 1.1 shows the general location of SRS. SRS is owned by DOE and is operated through management and operating contracts.

The mission of SRS is to serve local and national interests by applying its technical, physical, and human resources to protect and improve environmental quality, support a secure national defense and reduce the nuclear danger, and enhance industrial competitiveness and economic development in a safe, environmentally sound, socially responsible, and cost-effective manner in partnership with its stakeholders.

Recent Site mission changes have reduced the need for nuclear material production at SRS and heightened the need for environmental restoration and decontamination and decommissioning (D\&D) activities. However, there will be continued operation of the tritium, separations, and certain plutonium operations as well as analytical support activities.

Tritium requirements and the need for special isotopes, such as $\mathrm{Pu}^{238}$, dominate anticipated demand for separations operations for nuclear materials processing. SRS was the sole source of tritium required to maintain the nuclear weapons stockpile. Recycling and reloading of tritium is a continuing Site mission. Another mission for SRS is the processing of $\mathrm{Pu}^{238}$ used in radioisotopic thermal generators to provide electrical power for space missions.

Existing plutonium-bearing materials are being stored at SRS awaiting final disposition.

\subsubsection{Savannah River Site Principal Operations}

Historically, SRS produced nuclear materials by manufacturing fuel and target components, irradiating the components in nuclear reactors, and chemically extracting the desired nuclear materials from the irradiated fuel and targets.

The largest SRS facilities were for production. These facilities include the fuel and target component manufacturing complex in $\mathrm{M}$ Area; the production reactors located in $\mathrm{P}, \mathrm{K}, \mathrm{L}, \mathrm{C}$, and $\mathrm{R}$ Areas; and the separations process lines in $\mathrm{F}$ and $\mathrm{H}$ Areas. The M-Area production facilities and the reactors are not operating, and there are no plans to resume their operations. Separations canyon facilities are fully operational and have been selectively operated recently depending on the need. Recently, HB Line has operated to provide $\mathrm{Pu}^{238}$ in support of the National Aeronautics and Space Administration (NASA) and to process plutonium in storage to produce a more stable material.

Other major facilities are used to manage wastes. The largest, DWPF, began treating high-level liquid waste on March 7, 1996.

A major generator of mixed waste at SRS was the preparation, in M Area, of target and fuel assemblies for the reactors. This process was similar to a commercial metal-forming and finishing operation. Mixed wastes were generated from the electroplating operations and the creation of waste nickel-plating solutions after M-Area metal-forming and finishing facilities were shut down.

The major types of radionuclide recovery in the Separations area were $\mathrm{Pu}^{239}$ recovery, uranium-235 $\left(\mathrm{U}^{235}\right)$ and neptunium-237 $\left(\mathrm{Np}^{237}\right)$ recovery, and tritium recovery. The liquid high-level waste remaining after the nuclear materials are recovered in both Separations canyon facilities is made alkaline ( $\mathrm{pH} \mathrm{10-13)}$ and transferred by gravity to the F-Area and H-Area High-Level Radioactive Waste (HLW) Tank Farms. High pH is maintained to prevent corrosion of the carbon steel tanks. The waste liquid is a major mixed waste component at SRS.

Tritium is recovered in a complex of buildings in $\mathrm{H}$ Area. Tritium was extracted from irradiated lithiumaluminum targets until September, 1999. Future extractions will use different target material and will be performed at the new Tritium Extraction Facility in FY06. Tritium is also recycled from reservoirs removed 
from weapons in the field. Old reservoirs are refurbished and refilled as necessary. Mixed waste is generated from these operations.

SRS also contains many production support and research and development facilities including powerhouses, laboratories, administrative, and support facilities. Figure 1.2 shows the location of major production, support, and research and development areas at SRS.

\subsubsection{Savannah River Site Principal Mixed Waste Facilities}

The existing facilities that manage mixed waste are the F-Area and H-Area High-Level Waste (HLW) Tank Farms, the F/H ETP, the Mixed Waste Storage Shed (Building 316-M), the Mixed Waste Storage Pad (Building 315-4M), the Savannah River National Laboratory (SRNL) Mixed Waste Storage Tanks (MWST), New Solvent Storage Tanks (H33-H36), the TRU Waste Storage Pads, the Mixed Waste Storage Buildings (MWSB) (Buildings 643-29E and 643-43E), the Hazardous Waste Storage Facility (HWSF) (645-N, 645-2N, and 645-4N), the Solid Waste Storage Pads (SWSP), the DWPF Vitrification Facility, the DWPF Organic Waste Storage Tank (OWST) (closed), and the Z-Area Saltstone Processing Facility (standby).

Liquid HLW generated by the Separations facilities is stored in underground tanks in the F-Area and H-Area HLW Tank Farms. Waste must be stored prior to treatment to allow radioactive decay to reduce the radionuclide contamination to a safer level for processing. To reduce the volume of HLW in storage, the liquid waste containing metals, salts, and fission products from reactor processing is routed through evaporators. The evaporator overheads are piped to the F/H ETP where they are treated by a series of physical/chemical treatment steps that include $\mathrm{pH}$ adjustment, submicro filtration, reverse osmosis, and ion exchange. Treated effluent is discharged to surface water as authorized by a National Pollutant Discharge Elimination System (NPDES) permit. This system also treats contaminated cooling water and storm water prior to release.

Treatment residues from the F/H ETP processes and the low-level radioactive portion (decontaminated salt solution) of the high-level liquid radioactive wastes in the F-Area and H-Area HLW Tank Farm are piped to the Z-Area Saltstone Processing and Disposal Facility. This waste stream is mixed waste due to its corrosivity and potential to exceed the Toxicity Characteristic Leaching Procedure (TCLP) limits. The waste stream is stabilized by mixing with grout and flyash to create saltstone. The nonhazardous saltstone is disposed in the Z-Area Vaults.

The remainder of the high-level waste, salt slurry and sludge, will be mixed with glass frit and stabilized in borosilicate glass at the DWPF.

Another treatment facility at SRS is the SRNL MWST, where high and low activity waste streams from SRNL undergo neutralization and ion exchange to remove hazardous characteristics before receiving further processing at the F-Area HLW Tank Farm.

Mixed wastes are stored on the TRU pads, in the MWSB, in the HWSF, on the SWSP, on the M-Area storage pad, in storage tanks, and the Mixed Waste Storage Shed until they can be sent to the appropriate treatment and disposal facilities.

The site treatment plan and the annual update analyze treatment options for mixed waste using these facilities, with and without modifications, and investigate other options for treatment of mixed waste streams generated at SRS. 


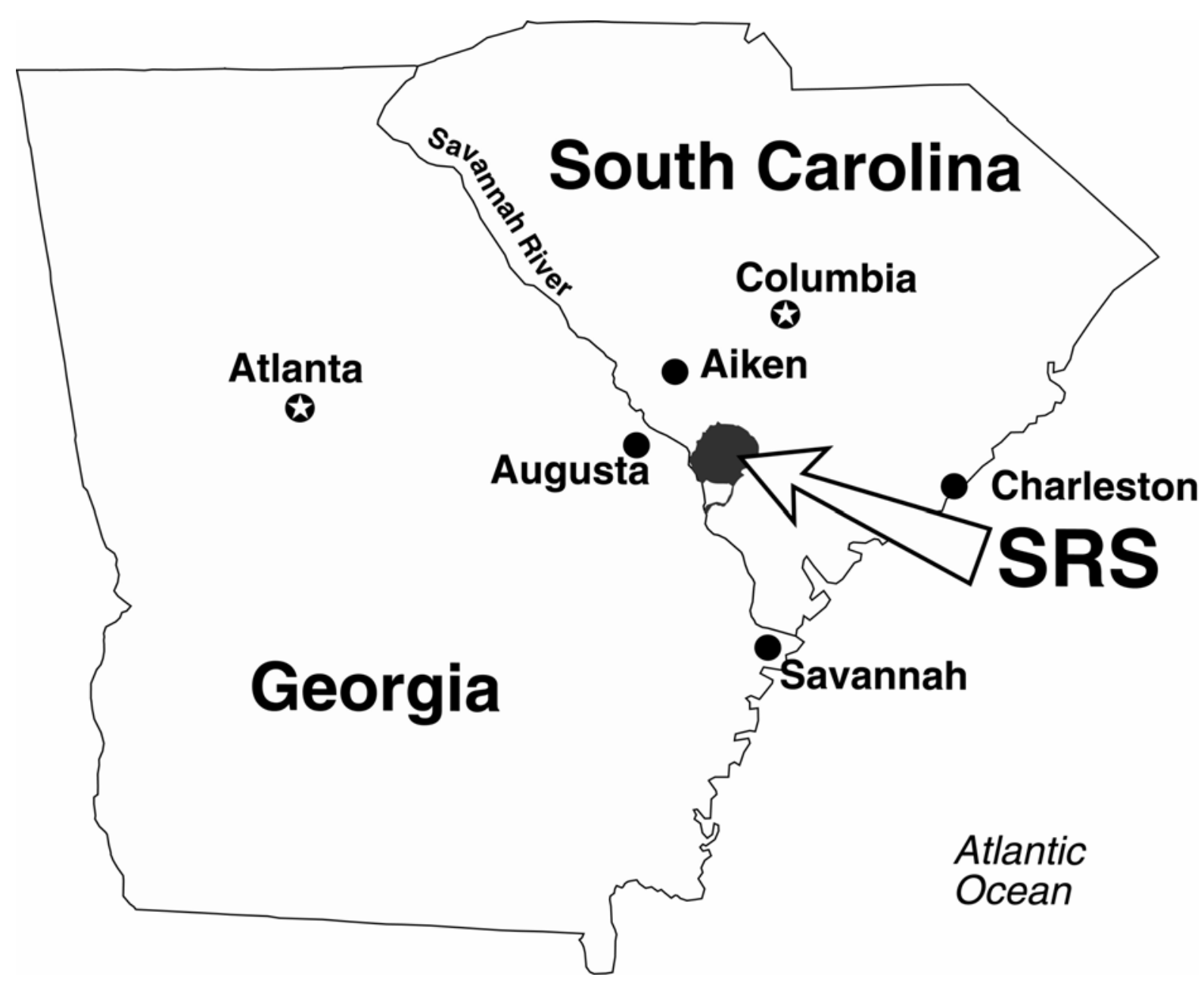

Figure 1.1 - General Location of Savannah River Site 


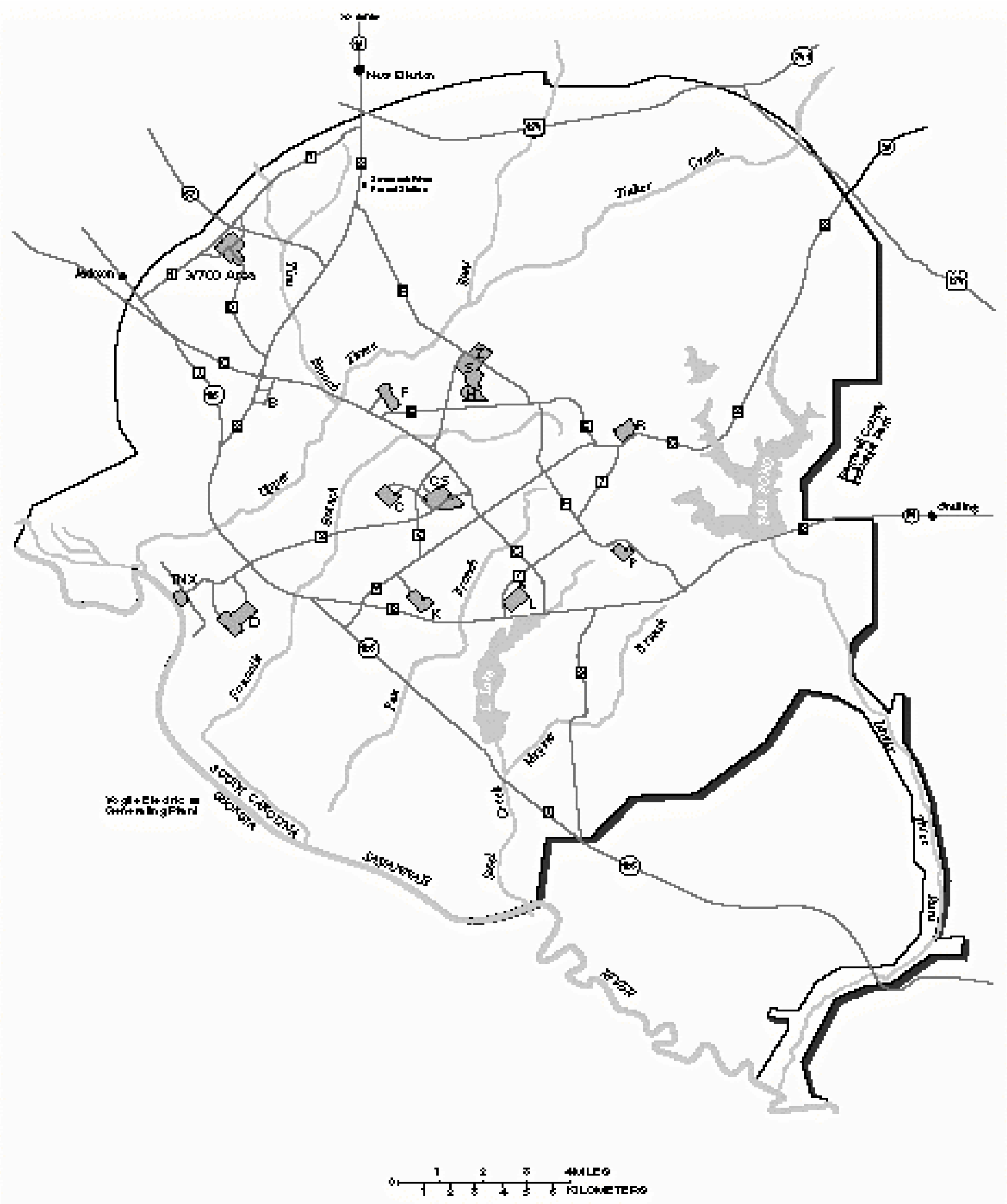

Figure 1.2 - Location of Major Production, Support, and Research and Development Areas at the Savannah River Site 


\subsection{STP Organization}

The STP and annual update are organized in two separate, but integrated, volumes. The Background Volume, Volume II, provides the detailed discussion of the options. It contains information on the waste streams and treatability groups a particular treatment option or options would address and describes uncertainties associated with that option, as well as the budget status of the option and regulator and stakeholder input. The Compliance Plan Volume, Volume I, is a short, focused document containing the preferred options and schedules for implementing the options and contains all the information required by the FFCAct. The Compliance Plan Volume also contains a mechanism to implement the plan and establish milestones enforced by the Order. It references, but does not duplicate, details on the options listed in the Background Volume.

Chapters 1 and 2 in both volumes contain introductory material relevant to the purpose of the volume. Chapters 1 and 2 of the Compliance Plan Volume contain certain administrative provisions appropriate for implementing the plan, such as project activity schedules describing funding considerations.

Chapters 3 through 5 discuss the preferred option for low-level mixed waste, mixed transuranic waste, and mixed high-level waste, and each volume discusses the same waste streams and options in parallel. The Background Volume discusses the waste streams, technology needs, and uncertainties and other details on the preferred options; the Compliance Plan Volume includes schedules, to the extent feasible, as required under the FFCAct.

The Background Volume includes seven additional sections not included in the Compliance Plan Volume. Chapter 6 of the Background Volume discusses mixed wastes expected to be generated from future activities such as environmental restoration and D\&D actions. These waste streams will be incorporated into the Compliance Volume, and treatment approaches and schedules developed, when the wastes are generated.

Chapter 8 describes the process being followed by DOE and the states for evaluating options for disposal of mixed waste treatment residues.

Chapter 9 provides a description of all existing treatment facilities at SRS for the treatment of mixed wastes.

Chapter 10 provides information on offsite waste from the Naval Reactors Program that lists SRS as the preferred treatment option. Final decisions on actual treatment were made by the requesting DOE site, SRS, DOE-HQ, affected states, and other stakeholders in the course of negotiations leading to the development of the consent order.

Chapter 11 provides summary information in three tables. Table 11.1 lists SRS mixed waste streams, their preferred treatment options, and currently generated volume. Table 11.2 provides the same information but lists waste streams by treatment facility or treatment method. Table 11.3 provides the volumes and status of mixed waste treatment residues resulting from the treatment of wastes listed in the STP.

Chapter 12 is a list of acronyms and definitions for terms used in the STP and annual updates.

The following table (Table 1.1) is a user's guide that is provided as an aid in reviewing waste stream information in the STP. Table 1.1 provides the status of each waste stream, the preferred treatment option (PO), and the primary locations of the waste streams in Volumes I and II of the STP. 
Table 1.1 User's Guide to Chapters 3, 4, and 5

\begin{tabular}{|c|c|c|c|c|c|}
\hline $\begin{array}{c}\text { Waste Stream } \\
\text { No. }\end{array}$ & $\begin{array}{l}\text { Waste Stream } \\
\text { Name }\end{array}$ & $\begin{array}{l}\text { Preferred Option } \\
\text { (PO) }\end{array}$ & $\begin{array}{l}\text { Volume I Section } \\
\text { Identification }\end{array}$ & $\begin{array}{c}\text { Volume II } \\
\text { Section } \\
\text { Identification }\end{array}$ & Waste Stream Status \\
\hline SR-W001 & $\begin{array}{l}\text { Rad-Contaminated } \\
\text { Solvents } \\
\end{array}$ & $\begin{array}{c}\text { Combustion at } \\
\text { Commercial Facility }\end{array}$ & 3.1 .2 .6 & 3.1 .2 .6 & Ongoing Treatment \\
\hline SR-W002 & $\begin{array}{c}\text { Rad-Contaminated } \\
\text { Chlorofluorocarbons }\end{array}$ & $\begin{array}{l}\text { Consolidated with } \\
\text { SR-W001 }\end{array}$ & N/A & 1.4, Table 1.1 & $\begin{array}{c}\text { Consolidated with SR- } \\
\text { W001 }\end{array}$ \\
\hline SR-W003 & $\begin{array}{c}\text { Solvent } \\
\text { Contaminated Debris } \\
\text { (LLW) }\end{array}$ & $\begin{array}{c}\text { Thermal Treatment, } \\
\text { Macroencapsulation } \\
\text { or Stabilization and/or } \\
\text { alternative debris } \\
\text { treatment at } \\
\text { Commercial Vendor } \\
\text { Facility }\end{array}$ & 3.1 .2 .6 & 3.1 .2 .6 & Ongoing Treatment \\
\hline SR-W004 & $\begin{array}{l}\text { M-Area Plating Line } \\
\text { Sludge from } \\
\text { Supernate Treatment }\end{array}$ & $\begin{array}{l}\text { Consolidated with } \\
\text { SR-W037 }\end{array}$ & N/A & 1.4 , Table 1.1 & $\begin{array}{c}\text { Consolidated with SR- } \\
\text { W037 }\end{array}$ \\
\hline SR-W005 & Mark 15 Filtercake & $\begin{array}{c}\text { Stabilization by } \\
\text { Vitrification- } \\
\text { M-Area Vendor } \\
\text { Treatment Facility }\end{array}$ & N/A & 3.5 .3 & Waste Stream Treated \\
\hline SR-W006 & $\begin{array}{c}\text { CH Mixed } \\
\text { TRU/Liquids }\end{array}$ & $\begin{array}{c}\text { Characterization at } \\
\text { SRS-WIPP Disposal }\end{array}$ & 4.1 & 4.2 .2 & $\begin{array}{c}\text { Awaiting } \\
\text { Characterization }\end{array}$ \\
\hline SR-W007 & $\begin{array}{c}\text { SRNL Sample } \\
\text { Material* }\end{array}$ & $\begin{array}{l}\text { Waste Stream } \\
\text { Eliminated* }\end{array}$ & N/A & $\begin{array}{l}\text { Appendix to } \\
\text { Chapter } 3\end{array}$ & $\begin{array}{l}\text { Waste Stream } \\
\text { Eliminated* }\end{array}$ \\
\hline SR-W008 & $\begin{array}{c}\text { Separations Area } \\
\text { Sample Receipts } \\
\text { from SRNL* }\end{array}$ & $\begin{array}{c}\text { Vitrification, } \\
\text { Stabilization, or WW } \\
\text { Treatment* }\end{array}$ & N/A & $\begin{array}{l}\text { Appendix to } \\
\text { Chapter } 3\end{array}$ & Ongoing Treatment* \\
\hline SR-W009 & $\begin{array}{c}\text { Silver Coated } \\
\text { Packing Material }\end{array}$ & $\begin{array}{c}\text { Macroencapsulation } \\
\text { in a Steel Container- } \\
\text { Onsite via a } \\
\text { Treatability Variance }\end{array}$ & 3.1 .1 .3 & 3.1 .1 .3 & $\begin{array}{c}\text { Awaiting Variance } \\
\text { Approval }\end{array}$ \\
\hline SR-W010 & Scintillation Solution & $\begin{array}{l}\text { Consolidated with } \\
\text { SR-W001 }\end{array}$ & N/A & 1.4 , Table 1.1 & $\begin{array}{c}\text { Consolidated with SR- } \\
\text { W001 }\end{array}$ \\
\hline SR-W011 & $\begin{array}{l}\text { Cadmium Coated } \\
\text { HEPA Filters }\end{array}$ & $\begin{array}{l}\text { Scrap Metal } \\
\text { Exclusion }\end{array}$ & N/A & $\begin{array}{l}\text { Appendix to } \\
\text { Chapter } 3\end{array}$ & $\begin{array}{c}\text { Recycled under Scrap } \\
\text { Metal Exclusion }\end{array}$ \\
\hline SR-W012 & $\begin{array}{c}\text { Toxic Characteristic } \\
\text { Solids }\end{array}$ & $\begin{array}{c}\text { Thermal Treatment, } \\
\text { Macroencapsulation } \\
\text { or Stabilization and/or } \\
\text { alternative debris } \\
\text { treatment at } \\
\text { Commercial Facility }\end{array}$ & 3.1 .2 .6 & 3.1 .2 .6 & Ongoing Treatment \\
\hline SR-W013 & $\begin{array}{l}\text { Low-Level Waste } \\
\text { (LLW) Lead - To be } \\
\text { Decontaminated } \\
\text { Onsite }\end{array}$ & $\begin{array}{l}\text { Reclassified as } \\
\text { material in use }\end{array}$ & N/A & 3.5.4 & $\begin{array}{l}\text { Waste Stream } \\
\text { Eliminated }\end{array}$ \\
\hline SR-W014 & $\begin{array}{l}\text { Elemental (Liquid) } \\
\text { Mercury - Sitewide }\end{array}$ & $\begin{array}{c}\text { Amalgamation- } \\
\text { Offsite Commercial } \\
\text { Vendor } \\
\end{array}$ & 3.1 .2 .4 & 3.1 .2 .4 & Awaiting Treatment \\
\hline SR-W015 & $\begin{array}{c}\text { Tritium } \\
\text { Contaminated } \\
\text { Equipment } \\
\end{array}$ & $\begin{array}{c}\text { Macroencapsulation } \\
\text { in S. S. Container as } \\
\text { 90-Day Generator }\end{array}$ & N/A & $\begin{array}{l}\text { Appendix to } \\
\text { Chapter } 3\end{array}$ & Ongoing Treatment \\
\hline SR-W016 & $\begin{array}{l}\text { 221-F Canyon High- } \\
\text { Level Liquid Waste }\end{array}$ & Vitrification at DWPF & 5 & 5 & Ongoing Treatment \\
\hline
\end{tabular}


Table 1.1 User's Guide to Chapters 3, 4, and 5 (con't)

\begin{tabular}{|c|c|c|c|c|c|}
\hline $\begin{array}{c}\text { Waste Stream } \\
\text { No. }\end{array}$ & $\begin{array}{l}\text { Waste Stream } \\
\text { Name }\end{array}$ & $\begin{array}{l}\text { Preferred Option } \\
\text { (PO) }\end{array}$ & $\begin{array}{l}\text { Volume I Section } \\
\text { Identification }\end{array}$ & $\begin{array}{c}\text { Volume II } \\
\text { Section } \\
\text { Identification }\end{array}$ & $\begin{array}{c}\text { Waste Stream } \\
\text { Status }\end{array}$ \\
\hline SR-W017 & $\begin{array}{l}\text { 221-H Canyon High- } \\
\text { Level Liquid Waste }\end{array}$ & Vitrification at DWPF & 5 & 5 & $\begin{array}{l}\text { Ongoing } \\
\text { Treatment }\end{array}$ \\
\hline SR-W018 & $\begin{array}{l}\text { Listed Incinerable } \\
\text { Solids }\end{array}$ & $\begin{array}{c}\text { Combustion at } \\
\text { Commercial Facility or } \\
\text { Macroencapsulation or } \\
\text { Stabilization at } \\
\text { Commercial Facility }\end{array}$ & 3.1 .2 .6 & 3.1 .2 .6 & $\begin{array}{l}\text { Wastestream } \\
\text { Treated }\end{array}$ \\
\hline SR-W019 & $\begin{array}{c}\text { 244-H RBOF High } \\
\text { Activity Liquid Waste }\end{array}$ & $\begin{array}{l}\text { Consolidated with SR- } \\
\text { W017 }\end{array}$ & N/A & 1.4, Table 1.1 & $\begin{array}{l}\text { Consolidated } \\
\text { with SR-W017 }\end{array}$ \\
\hline SR-W020 & $\begin{array}{l}\text { Salt Processing } \\
\text { Filters }\end{array}$ & $\begin{array}{c}\text { Future Generation - } \\
\text { Treatment to be } \\
\text { Determined }\end{array}$ & N/A & $\begin{array}{r}\text { Appendix to } \\
\text { Chapter } 3\end{array}$ & $\begin{array}{l}\text { Future } \\
\text { Generation }\end{array}$ \\
\hline SR-W021 & $\begin{array}{c}\text { Poisoned Catalyst } \\
\text { Material }\end{array}$ & $\begin{array}{r}\text { Waste Stream } \\
\text { Eliminated }\end{array}$ & N/A & 1.4, Table 1.1 & $\begin{array}{r}\text { Waste Stream } \\
\text { Eliminated }\end{array}$ \\
\hline SR-W022 & DWPF Benzene & $\begin{array}{r}\text { Waste Stream } \\
\text { Eliminated } \\
\end{array}$ & N/A & 1.4 , Table 1.1 & $\begin{array}{r}\text { Waste Stream } \\
\text { Eliminated } \\
\end{array}$ \\
\hline SR-W023 & $\begin{array}{c}\text { Cadmium } \\
\text { Safety/Control Rods }\end{array}$ & $\begin{array}{c}\text { Macroencapsulation in a } \\
\text { Cask as a } \\
\text { 90-Day Generator }\end{array}$ & N/A & $\begin{array}{r}\text { Appendix to } \\
\text { Chapter } 3\end{array}$ & $\begin{array}{c}\text { Complies with } \\
\text { LDR }\end{array}$ \\
\hline SR-W024 & $\begin{array}{l}\text { Mercury/Tritium Gold } \\
\text { Traps }\end{array}$ & $\begin{array}{l}\text { Meets LDR Treatment } \\
\text { Standard }\end{array}$ & N/A & $\begin{array}{l}\text { Appendix to } \\
\text { Chapter } 3\end{array}$ & $\begin{array}{l}\text { Complies with } \\
\text { LDR }\end{array}$ \\
\hline SR-W025 & $\begin{array}{l}\text { Solvent/TRU Job } \\
\text { Control Waste } \\
<100 \mathrm{nCi} / \mathrm{g}\end{array}$ & $\begin{array}{l}\text { Consolidated with SR- } \\
\text { W027* }\end{array}$ & N/A* & 1.4 , Table $1.1^{*}$ & $\begin{array}{c}\text { Consolidated } \\
\text { with SR-W027* }\end{array}$ \\
\hline SR-W026 & CH Mixed TRU/Thirds & $\begin{array}{l}\text { Characterization at } \\
\text { SRS-WIPP Disposal }\end{array}$ & 4.1 & 4.2 .1 .1 & $\begin{array}{l}\text { Awaiting } \\
\text { Treatment }\end{array}$ \\
\hline SR-W027 & $\begin{array}{l}\text { CH Mixed TRU/F- } \\
\text { Listed Solvents }\end{array}$ & $\begin{array}{l}\text { Characterization at } \\
\text { SRS-WIPP Disposal }\end{array}$ & 4.1 & 4.2 .1 .1 & $\begin{array}{l}\text { Awaiting } \\
\text { Treatment }\end{array}$ \\
\hline SR-W028 & Mark 15 Filter Paper & $\begin{array}{c}\text { Combustion at } \\
\text { Commercial Facility or } \\
\text { Macroencapsulation or } \\
\text { Stabilization at } \\
\text { Commercial Facility }\end{array}$ & N/A & 3.5 .1 & $\begin{array}{l}\text { Waste Stream } \\
\text { Treated }\end{array}$ \\
\hline SR-W029 & $\begin{array}{c}\text { M-Area Sludge } \\
\text { Treatability Samples }\end{array}$ & Waste Stream Treated & N/A & 3.5 .3 & $\begin{array}{l}\text { Waste Stream } \\
\text { Treated }\end{array}$ \\
\hline SR-W030 & $\begin{array}{l}\text { Spent Methanol } \\
\text { Solution }\end{array}$ & $\begin{array}{l}\text { Consolidated with SR- } \\
\text { W001 }\end{array}$ & N/A & 1.4 , Table 1.1 & $\begin{array}{c}\text { Consolidated } \\
\text { with SR-W001 }\end{array}$ \\
\hline SR-W031 & $\begin{array}{l}\text { Uranium/Chromium } \\
\text { Solution }\end{array}$ & $\begin{array}{c}\text { Stabilization by } \\
\text { Vitrification-M-Area } \\
\text { Vendor Treatment } \\
\text { Facility } \\
\end{array}$ & N/A & 3.5 .3 & $\begin{array}{l}\text { Waste Stream } \\
\text { Treated }\end{array}$ \\
\hline SR-W032A & $\begin{array}{c}\text { Mercury-Contaminated } \\
\text { Heavy Water }\end{array}$ & $\begin{array}{l}\text { Waste Stream } \\
\text { Eliminated }\end{array}$ & N/A & 3.5 .2 & $\begin{array}{c}\text { Treated to meet } \\
\text { LDR }\end{array}$ \\
\hline SR-W032B & $\begin{array}{l}\text { Mercury-Contaminated } \\
\text { Heavy Water Residues }\end{array}$ & $\begin{array}{c}\text { Solidification in } \\
\text { container as a 90-day } \\
\text { generator }\end{array}$ & N/A & $\begin{array}{l}\text { Appendix to } \\
\text { Chapter } 3\end{array}$ & $\begin{array}{l}\text { Complies with } \\
\text { LDR }\end{array}$ \\
\hline SR-W033 & $\begin{array}{c}\text { Thirds/TRU Job } \\
\text { Control Waste } \\
<100 \mathrm{nCi} / \mathrm{g} \\
\end{array}$ & $\begin{array}{c}\text { Consolidated with SR- } \\
\text { W026* }\end{array}$ & N/A* & 1.4 , Table $1.1^{*}$ & $\begin{array}{c}\text { Consolidated } \\
\text { with SR-W026* }\end{array}$ \\
\hline SR-W034 & Calcium Metal & $\begin{array}{c}\text { Deactivation by } \\
\text { commercial Vendor }\end{array}$ & 3.1 .2 .7 & 3.1 .2 .7 & $\begin{array}{l}\text { Awaiting } \\
\text { Treatment }\end{array}$ \\
\hline
\end{tabular}


Table 1.1 User's Guide to Chapters 3, 4, and 5 (cont'd)

\begin{tabular}{|c|c|c|c|c|c|}
\hline $\begin{array}{l}\text { Waste Stream } \\
\text { No. }\end{array}$ & Waste Stream Name & Preferred Option (PO) & $\begin{array}{l}\text { Volume I Section } \\
\text { Identification }\end{array}$ & $\begin{array}{c}\text { Volume II } \\
\text { Section } \\
\text { Identification } \\
\end{array}$ & $\begin{array}{l}\text { Waste Stream } \\
\text { Status }\end{array}$ \\
\hline SR-W035 & $\begin{array}{l}\text { Mixed Waste Oil- } \\
\text { Sitewide }\end{array}$ & $\begin{array}{l}\text { Thermal Treatment at } \\
\text { Commercial Facility }\end{array}$ & 3.1 .2 .6 & 3.1 .2 .6 & $\begin{array}{c}\text { Ongoing } \\
\text { Treatment }\end{array}$ \\
\hline SR-W036 & $\begin{array}{l}\text { Tritiated Oil with } \\
\text { Mercury }\end{array}$ & $\begin{array}{c}\text { Treatment by Aging } \\
\text { followed by Combustion }\end{array}$ & N/A & 3.4 & $\begin{array}{l}\text { Awaiting } \\
\text { Treatment }\end{array}$ \\
\hline SR-W037 & $\begin{array}{l}\text { M-Area Plating Line } \\
\text { Sludges }\end{array}$ & $\begin{array}{c}\text { Stabilization by } \\
\text { Vitrification M-Area } \\
\text { Vendor Treatment } \\
\text { Facility }\end{array}$ & N/A & 3.5 .3 & $\begin{array}{l}\text { Waste Stream } \\
\text { Treated }\end{array}$ \\
\hline SR-W038 & $\begin{array}{l}\text { Plating Line Sump } \\
\text { Material }\end{array}$ & $\begin{array}{c}\text { Stabilization by } \\
\text { Vitrification M-Area } \\
\text { Vendor Treatment } \\
\text { Facility }\end{array}$ & N/A & 3.5 .3 & $\begin{array}{l}\text { Waste Stream } \\
\text { Treated }\end{array}$ \\
\hline SR-W039 & $\begin{array}{l}\text { Nickel Plating Line } \\
\text { Solution }\end{array}$ & $\begin{array}{c}\text { Stabilization by } \\
\text { Vitrification M-Area } \\
\text { Vendor Treatment } \\
\text { Facility }\end{array}$ & N/A & 3.5 .3 & $\begin{array}{l}\text { Waste Stream } \\
\text { Treated }\end{array}$ \\
\hline SR-W040 & \begin{tabular}{c|} 
Listed Stabilized \\
Sludge/Listed LDR \\
- Compliant Debris
\end{tabular} & $\begin{array}{c}\text { Waste Stream } \\
\text { Treated in } \\
\text { Compliance with } \\
\text { LDR } \\
\end{array}$ & N/A & $\begin{array}{l}\text { Appendix to } \\
\text { Chapter } 3\end{array}$ & $\begin{array}{l}\text { Complies } \\
\text { with LDR }\end{array}$ \\
\hline SR-W041 & $\begin{array}{l}\text { Aqueous Mercury } \\
\text { and Lead }\end{array}$ & $\begin{array}{l}\text { Effluent Treatment } \\
\text { Project }\end{array}$ & N/A & $\begin{array}{l}\text { Appendix to } \\
\text { Chapter } 3\end{array}$ & $\begin{array}{l}\text { Treated to } \\
\text { meet LDR }\end{array}$ \\
\hline SR-W042 & Paints and Thinners & $\begin{array}{c}\text { Thermal Treatment } \\
\text { Macroencapsulation } \\
\text { or Stabilization at } \\
\text { Commercial Facility }\end{array}$ & 3.1 .2 .6 & 3.1 .2 .6 & $\begin{array}{l}\text { Ongoing } \\
\text { Treatment }\end{array}$ \\
\hline SR-W043 & $\begin{array}{l}\text { Lab Waste with } \\
\text { Tetraphenyl Borate }\end{array}$ & $\begin{array}{l}\text { Consolidated with } \\
\text { SR-W012 }\end{array}$ & N/A & 1.4 , Table 1.1 & $\begin{array}{c}\text { Consolidated } \\
\text { with SR-W012 }\end{array}$ \\
\hline SR-W044 & $\begin{array}{c}\text { Tri-Butyl- } \\
\text { Phosphate \& n- } \\
\text { Paraffin-TRU }\end{array}$ & $\begin{array}{l}\text { Consolidated with } \\
\text { SR-W045A \& B }\end{array}$ & $\mathrm{N} / \mathrm{A}$ & 1.4, Table 1.1 & $\begin{array}{c}\text { Consolidated } \\
\text { with SR-W045 }\end{array}$ \\
\hline SR-W045A & $\begin{array}{l}\text { PUREX Aqueous } \\
\text { Waste }\end{array}$ & $\begin{array}{l}\text { Waste Stream } \\
\text { Eliminated }\end{array}$ & N/A & 1.4, Table 1.1 & $\begin{array}{c}\text { Waste } \\
\text { Stream } \\
\text { Eliminated }\end{array}$ \\
\hline SR-W045 & PUREX Waste & $\begin{array}{l}\text { Solidification using } \\
\text { new technology }\end{array}$ & 3.2 .3 & 3.2 .3 & $\begin{array}{l}\text { Awaiting } \\
\text { Treatment }\end{array}$ \\
\hline SR-W046 & $\begin{array}{l}\text { Consolidated } \\
\text { Incineration } \\
\text { Facility Ash }\end{array}$ & $\begin{array}{l}\text { Waste stream } \\
\text { eliminated }\end{array}$ & N/A & $\begin{array}{l}\text { Appendix to } \\
\text { Chapter } 3\end{array}$ & $\begin{array}{l}\text { Waste Stream } \\
\text { Treated }\end{array}$ \\
\hline SR-W047 & $\begin{array}{c}\text { Consolidated } \\
\text { Incineration Facility } \\
\text { Blowdown }\end{array}$ & Waste stream eliminated & N/A & $\begin{array}{l}\text { Appendix to } \\
\text { Chapter } 3\end{array}$ & $\begin{array}{l}\text { Waste Stream } \\
\text { Treated }\end{array}$ \\
\hline SR-W048 & $\begin{array}{l}\text { Soils from Spill } \\
\text { Remediation }\end{array}$ & $\begin{array}{c}\text { Stabilization by } \\
\text { Commercial Vendor }\end{array}$ & 3.1 .2 .5 & 3.1 .2 .5 & $\begin{array}{c}\text { Ongoing } \\
\text { Treatment }\end{array}$ \\
\hline SR-W049 & $\begin{array}{c}\text { Tank E-3-1 Clean Out } \\
\text { Material } \\
\end{array}$ & Waste stream eliminated & N/A & 1.4 , Table 1.1 & $\begin{array}{c}\text { Waste Stream } \\
\text { Treated } \\
\end{array}$ \\
\hline SR-W050 & $\begin{array}{c}\text { Waste to Support High- } \\
\text { Level Waste (HLW) } \\
\text { Processing } \\
\text { Demonstrations } \\
\end{array}$ & $\begin{array}{c}\text { Treatment by SRNL as a } \\
\text { 90-Day Generator }\end{array}$ & N/A & $\begin{array}{l}\text { Appendix to } \\
\text { Chapter } 3\end{array}$ & $\begin{array}{l}\text { Complies with } \\
\text { LDR }\end{array}$ \\
\hline
\end{tabular}


Table 1.1 User's Guide to Chapters 3, 4, and 5 (cont'd)

\begin{tabular}{|c|c|c|c|c|c|}
\hline $\begin{array}{c}\text { Waste Stream } \\
\text { No. }\end{array}$ & $\begin{array}{l}\text { Waste Stream } \\
\text { Name }\end{array}$ & $\begin{array}{l}\text { Preferred Option } \\
\text { (PO) }\end{array}$ & $\begin{array}{l}\text { Volume I Section } \\
\text { Identification }\end{array}$ & $\begin{array}{c}\text { Volume II } \\
\text { Section } \\
\text { Identification }\end{array}$ & $\begin{array}{c}\text { Waste Stream } \\
\text { Status }\end{array}$ \\
\hline SR-W051 & $\begin{array}{c}\text { Spent Filter Cartridges } \\
\text { and Carbon Filter } \\
\text { Media }\end{array}$ & $\begin{array}{l}\text { Thermal Treatment, } \\
\text { Macroencapsulation or } \\
\text { Stabilization and/or } \\
\text { alternative debris } \\
\text { treatment at Commercial } \\
\text { Facility }\end{array}$ & 3.1 .2 .6 & 3.1 .2 .6 & $\begin{array}{l}\text { Awaiting } \\
\text { Treatment }\end{array}$ \\
\hline SR-W052 & $\begin{array}{c}\text { Cadmium- } \\
\text { Contaminated } \\
\text { Glovebox Section }\end{array}$ & $\begin{array}{l}\text { Waste Stream } \\
\text { Eliminated }\end{array}$ & N/A & 1.4, Table 1.1 & $\begin{array}{l}\text { Waste Stream } \\
\text { Eliminated }\end{array}$ \\
\hline SR-W053 & Rocky Flats Ash & $\begin{array}{l}\text { Characterization at } \\
\text { SRS-Ship to WIPP }\end{array}$ & 4.2 .1 & 4.2 .2 & $\begin{array}{l}\text { Awaiting } \\
\text { Treatment }\end{array}$ \\
\hline SR-W054 & $\begin{array}{c}\text { Enriched Uranium } \\
\text { Contaminated with } \\
\text { Lead }\end{array}$ & $\begin{array}{l}\text { Consolidated with } \\
\text { SR-W037 }\end{array}$ & N/A & 1.4, Table 1.1 & $\begin{array}{c}\text { Consolidated } \\
\text { with SR-W037 }\end{array}$ \\
\hline SR-W055 & $\begin{array}{c}\text { Job Control Waste } \\
\text { Containing Solvent } \\
\text { Contaminated Wipes }\end{array}$ & $\begin{array}{c}\text { Thermal Treatment, } \\
\text { Macroencapsulation or } \\
\text { Stabilization at } \\
\text { Commercial Facility }\end{array}$ & 3.1 .2 .6 & 3.1 .2 .6 & $\begin{array}{c}\text { Ongoing } \\
\text { Treatment }\end{array}$ \\
\hline SR-W056 & $\begin{array}{c}\text { Job Control Waste } \\
\text { with Enriched Uranium } \\
\text { and Solvent } \\
\text { Contaminated Wipes }\end{array}$ & $\begin{array}{c}\text { Waste Stream } \\
\text { Re-characterized }\end{array}$ & N/A & 1.4 , Table 1.1 & $\begin{array}{l}\text { Waste Stream } \\
\text { Eliminated }\end{array}$ \\
\hline SR-W057 & $\begin{array}{l}\text { D-Tested Neutron } \\
\text { Generators }\end{array}$ & $\begin{array}{l}\text { Waste Stream } \\
\text { Eliminated }\end{array}$ & N/A & $\begin{array}{c}\text { 1.4, Table } \\
1.1\end{array}$ & $\begin{array}{l}\text { Waste Stream } \\
\text { Eliminated }\end{array}$ \\
\hline SR-W058 & $\begin{array}{c}\text { Mixed Sludge Waste } \\
\text { with Mercury from } \\
\text { DWPF Treatability } \\
\text { Studies }\end{array}$ & $\begin{array}{l}\text { Treatment by SRNL as } \\
\text { a 90-Day Generator }\end{array}$ & N/A & $\begin{array}{l}\text { Appendix to } \\
\text { Chapter } 3\end{array}$ & $\begin{array}{l}\text { Treated to meet } \\
\text { LDR }\end{array}$ \\
\hline SR-W059 & $\begin{array}{c}\text { Tetrabutyl Titanate } \\
\text { (TBT) }\end{array}$ & $\begin{array}{l}\text { Consolidated with SR- } \\
\text { W001 }\end{array}$ & N/A & $\begin{array}{c}1.4 \\
\text { Table } 1.1\end{array}$ & $\begin{array}{c}\text { Consolidated } \\
\text { with SR-W001 }\end{array}$ \\
\hline SR-W060 & $\begin{array}{l}\text { Tritiated Water with } \\
\text { Mercury }\end{array}$ & $\begin{array}{l}\text { Macroencapsulation in } \\
\text { a Steel Container via a } \\
\text { Treatability Variance }\end{array}$ & 3.1 .1 .3 & 3.1 .1 .3 & $\begin{array}{l}\text { Awaiting } \\
\text { Variance } \\
\text { Approval }\end{array}$ \\
\hline SR-W061 & DWPF Mercury & $\begin{array}{l}\text { Consolidated with SR- } \\
\text { W068 }\end{array}$ & N/A & $\begin{array}{c}1.4, \\
\text { Table } 1.1\end{array}$ & $\begin{array}{c}\text { Consolidated } \\
\text { with SR-W068 }\end{array}$ \\
\hline SR-W062 & $\begin{array}{l}\text { Normal Low-Level } \\
\text { Contaminated Debris }\end{array}$ & $\begin{array}{l}\text { Macroencapsulation or } \\
\text { stabilization by a } \\
\text { commercial vendor } \\
\text { and/or alternative } \\
\text { debris treatment at an } \\
\text { offsite facility }\end{array}$ & 3.1 .2 .2 & 3.1 .2 .2 & $\begin{array}{c}\text { Ongoing } \\
\text { Treatment }\end{array}$ \\
\hline SR-W063 & $\begin{array}{l}\text { Macroencapsulated } \\
\text { Low-Level Waste } \\
\text { (TC) }\end{array}$ & $\begin{array}{l}\text { Meets Treatment } \\
\text { Standard }\end{array}$ & N/A & $\begin{array}{l}\text { Appendix to } \\
\text { Chapter } 3\end{array}$ & $\begin{array}{l}\text { Complies with } \\
\text { LDR }\end{array}$ \\
\hline SR-W064 & $\begin{array}{c}\text { IDW Soils/Sludges/ } \\
\text { Slurries }\end{array}$ & Awaiting ROD, etc. & N/A & 6.1 & $\begin{array}{c}\text { Future } \\
\text { Generation }\end{array}$ \\
\hline SR-W065 & $\begin{array}{c}\text { IDW Monitoring } \\
\text { Well } \\
\text { Purge/Development } \\
\text { Water }\end{array}$ & Awaiting ROD, etc. & N/A & 6.1 & $\begin{array}{c}\text { Future } \\
\text { Generation }\end{array}$ \\
\hline SR-W066 & IDW Debris & Awaiting ROD, etc. & N/A & 6.1 & $\begin{array}{c}\text { Future } \\
\text { Generation }\end{array}$ \\
\hline
\end{tabular}


Table 1.1 User's Guide to Chapters 3, 4, and 5 (cont'd)

\begin{tabular}{|c|c|c|c|c|c|}
\hline $\begin{array}{c}\text { Waste Stream } \\
\text { No. }\end{array}$ & $\begin{array}{l}\text { Waste Stream } \\
\text { Name }\end{array}$ & $\begin{array}{l}\text { Preferred Option } \\
\text { (PO) }\end{array}$ & $\begin{array}{l}\text { Volume I Section } \\
\text { Identification }\end{array}$ & $\begin{array}{c}\text { Volume II } \\
\text { Section } \\
\text { Identification } \\
\end{array}$ & $\begin{array}{c}\text { Waste Stream } \\
\text { Status }\end{array}$ \\
\hline SR-W067 & $\begin{array}{c}\text { IDW Personal } \\
\text { Protective } \\
\text { Equipment (PPE) } \\
\text { Waste }\end{array}$ & Awaiting ROD, etc. & N/A & 6.1 & $\begin{array}{c}\text { Future } \\
\text { Generation }\end{array}$ \\
\hline SR-W068 & $\begin{array}{l}\text { Elemental (Liquid) } \\
\text { Mercury-Sitewide }\end{array}$ & $\begin{array}{l}\text { Consolidated with SR- } \\
\text { W014* }\end{array}$ & N/A* & 1.4, Table $1.1^{*}$ & $\begin{array}{c}\text { Consolidated } \\
\text { with SR-W014* }\end{array}$ \\
\hline SR-W069 & $\begin{array}{c}\text { Low-Level Waste } \\
\text { (LLW) Lead - To } \\
\text { be Macro- } \\
\text { encapsulated } \\
\text { Offsite } \\
\end{array}$ & $\begin{array}{l}\text { Macroencapsulation by } \\
\text { a commercial vendor at } \\
\text { an offsite facility }\end{array}$ & 3.1 .2 .3 & 3.1 .2 .3 & $\begin{array}{l}\text { Ongoing } \\
\text { Treatment }\end{array}$ \\
\hline SR-W070 & $\begin{array}{l}\text { Mixed Waste from } \\
\text { Laboratory Samples }\end{array}$ & $\begin{array}{c}\text { Combustion at } \\
\text { Commercial Vendor } \\
\text { Facility or } \\
\text { Macroencapsulation or } \\
\text { Stabilization at } \\
\text { Commercial Vendor } \\
\text { Facility } \\
\end{array}$ & 3.1 .2 .6 & 3.1 .2 .6 & $\begin{array}{l}\text { Awaiting } \\
\text { Treatment }\end{array}$ \\
\hline SR-W071 & \begin{tabular}{|c|} 
Wastewater \\
Suitable for Thermal \\
treatment or \\
Stabilization \\
\end{tabular} & $\begin{array}{c}\text { Thermal Treatment or } \\
\text { stabilization at } \\
\text { Commercial Vendor } \\
\text { Facility }\end{array}$ & 3.1 .2 .6 & 3.1 .2 .6 & $\begin{array}{l}\text { Ongoing } \\
\text { Treatment }\end{array}$ \\
\hline SR-W072 & $\begin{array}{c}\text { Supernate or } \\
\text { Sludge } \\
\text { Contaminated } \\
\text { Debris from High- } \\
\text { Level Waste (HLW) } \\
\text { Operations }\end{array}$ & $\begin{array}{c}\text { Extraction or } \\
\text { Immobilization } \\
\text { Alternative Debris } \\
\text { Technologies as a 90- } \\
\text { Day Generator }\end{array}$ & N/A & $\begin{array}{l}\text { Appendix to } \\
\text { Chapter } 3\end{array}$ & $\begin{array}{l}\text { Complies with } \\
\text { LDR }\end{array}$ \\
\hline SR-W073 & $\begin{array}{c}\text { Cadmium- } \\
\text { Containing Raschig } \\
\text { Rings }\end{array}$ & $\begin{array}{c}\text { Macroencapsulation by } \\
\text { Commercial Vendor } \\
\text { Offsite via Treatability } \\
\text { Variance or } \\
\text { stabilization at } \\
\text { Commercial Vendor } \\
\text { Facility } \\
\end{array}$ & 3.1 .2 .2 & 3.1 .2 .2 & $\begin{array}{l}\text { Awaiting } \\
\text { Treatment }\end{array}$ \\
\hline SR-W077 & $\begin{array}{c}\text { Aqueous } \\
\text { Characteristic } \\
\text { Wastewater }\end{array}$ & $\begin{array}{l}\text { Ion Exchange, Filtration, } \\
\text { nd/or Stabilization at F/H } \\
\text { ETP, Saltstone, or } \\
\text { Commercial Vendor } \\
\end{array}$ & N/A & $\begin{array}{l}\text { Appendix to } \\
\text { Chapter } 3\end{array}$ & $\begin{array}{r}\text { Ongoing } \\
\text { Treatment }\end{array}$ \\
\hline SR-W078 & $\begin{array}{l}\text { LDR Hazardous } \\
\text { Waste Awaiting } \\
\text { Radiological } \\
\text { Screening } \\
\end{array}$ & $\begin{array}{l}\text { Waste Stream } \\
\text { Eliminated }\end{array}$ & 3.3 .2 & 3.3 .2 & $\begin{array}{l}\text { Waste Stream } \\
\text { Eliminated }\end{array}$ \\
\hline SR-W079 & $\begin{array}{l}\text { Polychlorinated } \\
\text { Biphenyl (PCB) } \\
\text { Mixed Waste }\end{array}$ & $\begin{array}{l}\text { Combustion at the } \\
\text { ETTP TSCA } \\
\text { Incinerator or vendor } \\
\text { alternate debris } \\
\text { technology treatment }\end{array}$ & 3.1 .2 .1 & 3.1 .2 .1 & $\begin{array}{l}\text { Ongoing } \\
\text { Treatment }\end{array}$ \\
\hline $\begin{array}{c}\text { SR-W080* } \\
\text { (CN-W001, CN- } \\
\text { W004) }\end{array}$ & $\begin{array}{l}\text { Charleston Naval } \\
\text { Shipyard Waste }\end{array}$ & $\begin{array}{c}\text { Thermal Treatment or } \\
\text { Macroencapsulation or } \\
\text { Stabilization at } \\
\text { Commercial Vendor }\end{array}$ & 3.1 .2 .6 & 3.1 .2 .6 & $\begin{array}{l}\text { Awaiting } \\
\text { Treatment }\end{array}$ \\
\hline
\end{tabular}


Table 1.1 User's Guide to Chapters 3, 4, and 5 (cont'd)

\begin{tabular}{|c|c|c|c|c|c|}
\hline $\begin{array}{c}\text { Waste Stream } \\
\text { No. }\end{array}$ & $\begin{array}{c}\text { Waste Stream } \\
\text { Name }\end{array}$ & $\begin{array}{l}\text { Preferred Option } \\
\text { (PO) }\end{array}$ & $\begin{array}{l}\text { Volume I Section } \\
\text { Identification }\end{array}$ & $\begin{array}{c}\text { Volume II } \\
\text { Section } \\
\text { Identification }\end{array}$ & $\begin{array}{c}\text { Waste Stream } \\
\text { Status }\end{array}$ \\
\hline SR-W081 & $\begin{array}{c}\text { Reactive/Ignitable } \\
\text { Waste }\end{array}$ & $\begin{array}{c}\text { Deactivation } \\
\text { followed by } \\
\text { stabilization or } \\
\text { combustion at } \\
\text { Commercial Vendor }\end{array}$ & 3.1 .2 .6 & 3.1 .2 .6 & $\begin{array}{c}\text { Ongoing } \\
\text { Treatment }\end{array}$ \\
\hline SR-W082 & $\begin{array}{c}\text { Radioactive } \\
\text { Chemicals, Metals, } \\
\text { and Pesticides (CMP) } \\
\text { Soil }\end{array}$ & $\begin{array}{c}\text { Stabilization by } \\
\text { Commercial Vendor }\end{array}$ & 3.1 .2 .5 & 3.1 .2 .5 & $\begin{array}{l}\text { Ongoing } \\
\text { Treatment }\end{array}$ \\
\hline SR-W083 & $\begin{array}{l}\text { Mercury- and } \\
\text { Chromium- } \\
\text { Contaminated } \\
\text { Residues } \\
\end{array}$ & $\begin{array}{l}\text { Waste Stream Re- } \\
\text { Characterized }\end{array}$ & N/A & 1.4, Table 1.1 & $\begin{array}{l}\text { Waste Stream } \\
\text { Eliminated }\end{array}$ \\
\hline SR-W084 & $\begin{array}{l}\text { Remediation Waste } \\
\text { Soils that Meet LDR }\end{array}$ & $\begin{array}{l}\text { Meets Treatment } \\
\text { Standard } \\
\end{array}$ & N/A & 1.4 , Table 1.1 & $\begin{array}{l}\text { Complies with } \\
\text { LDR }\end{array}$ \\
\hline SR-W085 & $\begin{array}{c}\text { Rocky Flats } \\
\text { Plutonium Fluoride } \\
\text { Residues }\end{array}$ & $\begin{array}{l}\text { Future Generation- } \\
\text { Treatment to be } \\
\text { Determined }\end{array}$ & N/A & 1.4 , Table 1.1 & $\begin{array}{c}\text { Future } \\
\text { Generation }\end{array}$ \\
\hline SR-W086 & $\begin{array}{l}\text { Characteristically } \\
\text { Hazardous Non- } \\
\text { Incinerable Solids }\end{array}$ & $\begin{array}{c}\text { Amalgamation at } \\
\text { Commercial Vendor* }\end{array}$ & 3.1 .2 .4 & 3.1 .2 .4 & $\begin{array}{l}\text { Awaiting } \\
\text { Treatment }\end{array}$ \\
\hline $\begin{array}{c}\text { SR-W087 } \\
\text { (BT-W035, part } \\
\text { of BT-W003) } \\
\end{array}$ & $\begin{array}{c}\text { Bettis Atomic Power } \\
\text { Laboratory } \\
\text { Contaminated Oil }\end{array}$ & $\begin{array}{l}\text { Thermal Treatment } \\
\text { or Stabilization at } \\
\text { Commercial Facility }\end{array}$ & 3.1 .2 .6 & 3.1 .2 .6 & $\begin{array}{l}\text { Awaiting } \\
\text { Treatment }\end{array}$ \\
\hline SR-W088 & $\begin{array}{l}\text { Sludges and Non- } \\
\text { Debris Solids* }\end{array}$ & $\begin{array}{c}\text { Stabilization by } \\
\text { Commercial Vendor }\end{array}$ & 3.1 .2 .5 & 3.1 .2 .5 & $\begin{array}{c}\text { Ongoing } \\
\text { Treatment }\end{array}$ \\
\hline SR-W089 & $\begin{array}{l}\text { TRU Mixed Waste } \\
\text { from Mound Site }\end{array}$ & $\begin{array}{c}\text { Characterization at } \\
\text { SRS - WIPP Disposal }\end{array}$ & 4.2 .2 & 4.2 .2 & $\begin{array}{c}\text { Awaiting } \\
\text { Treatment }\end{array}$ \\
\hline SR-W090 & $\begin{array}{l}\text { Elemental Mercury } \\
\text { - High Rad }\end{array}$ & $\begin{array}{c}\text { Treatment by aging } \\
\text { F/B mercury } \\
\text { treatment* }\end{array}$ & $3.4 *$ & $3.4^{*}$ & $\begin{array}{l}\text { Awaiting } \\
\text { Treatment }\end{array}$ \\
\hline SR-W091* & $\begin{array}{c}\text { Contaminated } \\
\text { Debris (High Rad)* }\end{array}$ & $\begin{array}{l}\text { New Stream - } \\
\text { Treatment to be } \\
\text { Determined* }\end{array}$ & N/A* & $3.1 .3^{*}$ & $\begin{array}{c}\text { Awaiting } \\
\text { Treatment* }\end{array}$ \\
\hline
\end{tabular}

Some waste streams may be only briefly mentioned if they have been treated to meet LDR standards, recharacterized, or consolidated. Only the waste streams that require a schedule and a compliance order will be found in the Compliance Plan Volume. Waste streams not found in the Compliance Plan Volume have been recharacterized, combined, or are in compliance with applicable regulations.

* Indicates that a change has been made since last update. 
SRS Mixed Waste Streams included in Volume I of the Annual Update.

\begin{tabular}{|c|c|}
\hline SR-W001 & Rad-Contaminated Solvents \\
\hline SR-W003 & Solvent Contaminated Debris \\
\hline SR-W005 & Mark 15 Filtercake \\
\hline SR-W006 & CH Mixed TRU/Liquids \\
\hline SR-W009 & Silver Coated Packing Material \\
\hline SR-W012 & Toxic Characteristic Solids \\
\hline SR-W014 & Elemental (Liquid) Mercury - Sitewide \\
\hline SR-W016 & 221-F Canyon High-Level Liquid Waste \\
\hline SR-W017 & 221-H Canyon High-Level Liquid Waste \\
\hline SR-W018 & Listed Incinerable Solids \\
\hline SR-W025 & Solvent/TRU Job Control Waste $<100 \mathrm{nCi} / \mathrm{g}$ \\
\hline SR-W026 & CH Mixed TRU/Thirds \\
\hline SR-W027 & CH Mixed TRU/F-listed Solvents \\
\hline SR-W028 & Mark 15 Filter Paper \\
\hline SR-W029 & M-Area Sludge Treatability Samples \\
\hline SR-W031 & Uranium/Chromium Solution \\
\hline SR-W032A & Mercury Contaminated Heavy Water \\
\hline SR-W033 & Thirds/TRU Job Control Waste $<100 \mathrm{nCi} / \mathrm{g}$ \\
\hline SR-W034 & Calcium Metal \\
\hline SR-W035 & Mixed Waste Oil - Sitewide \\
\hline SR-W036 & Tritiated Oil with Mercury \\
\hline SR-W037 & M-Area Plating Line Sludges \\
\hline SR-W038 & Plating Line Sump Material \\
\hline SR-W039 & Nickel Plating Solution \\
\hline SR-W042 & Paints and Thinners \\
\hline SR-W045 & PUREX Waste \\
\hline SR-W048 & Soils from Spill Remediation \\
\hline SR-W051 & Spent Filter Cartridges and Carbon Filter Media \\
\hline SR-W053 & Rocky Flats Ash \\
\hline SR-W055 & Job Control Waste Containing Solvent Contaminated Wipes \\
\hline SR-W060 & Tritiated Water with Mercury \\
\hline SR-W062 & Normal Low-Level Contaminated Debris \\
\hline SR-W068 & Elemental (Liquid) Mercury - Sitewide \\
\hline SR-W069 & Low-Level Waste (LLW) Lead - to be Macroencapsulated Offsite \\
\hline SR-W070 & Mixed Waste from Laboratory Samples \\
\hline SR-W071 & Wastewater Suitable for Thermal treatment or Stabilization \\
\hline SR-W073 & Cadmium-Containing Raschig Rings \\
\hline SR-W079 & Polychlorinated Biphenyl (PCB) Mixed Waste \\
\hline SR-W081 & Reactive/Ignitable Waste \\
\hline SR-W082 & Radioactive Chemicals, Metals, and Pesticides (CMP) Soil \\
\hline SR-W086 & Characteristically Hazardous Non-Incinerable Solids \\
\hline
\end{tabular}


Offsite Waste Streams included in Volume I.

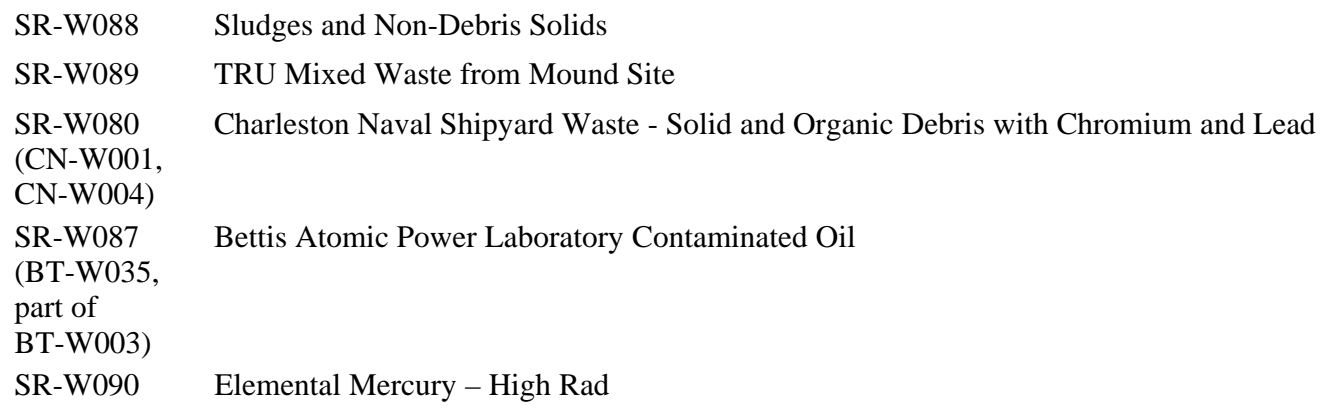

Waste streams that do not appear in the Compliance Plan Volume or the Background Volume because they have been eliminated as mixed waste.

$\begin{array}{ll}\text { SR-W013 } & \text { Low Level Waste Lead - To be decontaminated onsite } \\ \text { SR-W021 } & \text { Poisoned Catalyst Material } \\ \text { SR-W022 } & \text { DWPF Benzene } \\ \text { SR-W045A } & \text { PUREX Aqueous Waste } \\ \text { SR-W046 } & \text { Consolidated Incineration Facility Ash } \\ \text { SR-W047 } & \text { Consolidated Incineration Facility Blowdown } \\ \text { SR-W049 } & \text { Tank E-3-1 Clean Out Material } \\ \text { SR-W052 } & \text { Cadmium Contaminated Glovebox Section } \\ \text { SR-W056 } & \text { Job Control Waste with Enriched Uranium and Solvent Applicators } \\ \text { SR-W057 } & \text { D-Tested Neutron Generators } \\ \text { SR-W068 } & \text { Elemental (liquid) Mercury - Sitewide } \\ \text { SR-W083 } & \text { Mercury- and Chromium-Contaminated Residues }\end{array}$

Waste streams that do not appear in the Compliance Plan Volume preferred option discussion because they meet the Land Disposal Restrictions (LDR) Treatment Standard, meet the LDR standard when they are generated, or are recycled (includes scrap metal).

\begin{tabular}{|c|c|c|}
\hline SR-W011 & Cadmium-Coated HEPA Filters & Recycled under the scrap metal exclusion \\
\hline SR-W015 & Mercury/Tritium Contaminated Equipment & $\begin{array}{l}\text { Treated to meet LDR standard as a } \\
\text { 90-day generator }\end{array}$ \\
\hline SR-W023 & Cadmium Safety/Control Rods & $\begin{array}{l}\text { Treated to meet LDR standard as a } \\
\text { 90-day generator }\end{array}$ \\
\hline SR-W024 & Mercury/Tritium Gold Traps & Meets LDR treatment standard \\
\hline SR-W032B & Mercury-Contaminated Heavy Water Residues & $\begin{array}{l}\text { Treated to meet LDR treatment standards } \\
\text { as a } 90 \text {-day generator }\end{array}$ \\
\hline SR-W040 & Listed Stabilized Sludge/Listed LDR-Complaint Debris & Meets LDR treatment standard \\
\hline SR-W041 & Aqueous Mercury and Lead & $\begin{array}{l}\text { Treated to meet LDR standards - } \\
\text { May } 1995\end{array}$ \\
\hline SR-W050 & $\begin{array}{l}\text { Mixed Waste to Support High-Level Waste (HLW) } \\
\text { Processing Demonstrations }\end{array}$ & $\begin{array}{l}\text { Treated to meet LDR standards - } \\
\text { March } 1996\end{array}$ \\
\hline
\end{tabular}



SR-W058
Mixed Sludge Waste with Mercury from DWPF Treatability Studies
SR-W063 Macroencapsulated Toxic Characteristic (TC) Waste
SR-W072
SR-W077

To be treated to meet LDR standards as a 90-day generator in a containment building

Meets LDR treatment standard

To be treated to meet LDR standards in a 90-day staging area

Treated to meet LDR standards

Waste streams that do not appear in the Compliance Plan Volume or the Background Volume preferred option discussion because they have been consolidated with other waste streams.

$\begin{array}{ll}\text { SR-W002 } & \text { Rad-Contaminated Chlorofluorocarbons - Combined with SR-W001 } \\ \text { SR-W004 } & \text { M-Area Plating Line Sludge from Supernate Treatment - Combined with SR-W037 } \\ \text { SR-W010 } & \text { Scintillation Solution - Combined with SR-W001 } \\ \text { SR-W019 } & \text { 244-H RBOF High Activity Liquid Waste - Combined with SR-W017 } \\ \text { SR-W030 } & \text { Spent Methanol Solution - Combined with SR-W001 } \\ \text { SR-W043 } & \text { Lab Waste with Tetraphenyl Borate - Combined with SR-W012 } \\ \text { SR-W044 } & \text { Tri-Butyl-Phosphate \& n-Paraffin - TRU - Combined with SR-W045 } \\ \text { SR-W054 } & \text { Enriched Uranium Contaminated with Lead - Combined with SR-W037 } \\ \text { SR-W059 } & \text { Tetrabutyl Titanate (TBT) - Combined with SR-W001 } \\ \text { SR-W061 } & \text { DWPF Mercury - Combined with SR-W068 }\end{array}$

Waste streams that may be generated in the future.

SR-W064 IDW Soils/Sludges/Slurries

SR-W065 IDW Monitoring Well Purge/Development Water

SR-W066 IDW Debris

SR-W067 IDW Personal Protective Equipment (PPE) Waste

SR-W084 Remediation Waste Soils that Meet LDR

SR-W085 Rocky Flats Putonium Fluoride Residues

\section{Waste Stream Order}

Waste streams are arranged in the chapters by radioactivity type - mixed low-level waste (MLLW) streams in Chapter 3, mixed transuranic (MTRU) waste in Chapter 4, and high-level mixed waste in Chapter 5. Definitions for these terms can be found in Chapter 12, "Definitions," of Volume II. Table 1.2 provides EPA Hazardous Waste Codes with subcategories.

\section{Waste Stream Analysis Information}

For each waste stream with a proposed treatment option that is discussed in Chapter 3 through 5 of the STP, Volume II, the following is provided.

\section{General Information}

This section contains a description for each waste stream. Waste streams that have been deleted or consolidated have been noted in Table 1.1 and have no additional detail provided in Chapters 3-5. 
This section also provides the waste stream number and description of the determined preferred treatment option. Some of these waste streams did not undergo an in-depth option analysis in the STP because the analysis for these waste streams was performed as a part of the design work to justify a waste treatment facility project and to identify suitable waste streams for treatment.

It should be understood that no option identified in the STP as a preferred option is absolutely final. As treatment technology and input from the state or other stakeholders is received, the preferred option may change.

Mixed transuranic waste streams are designated for disposal in the Waste Isolation Pilot Plant (WIPP) and therefore will not undergo option analyses. These waste streams will be characterized, followed by preparation, shipment to, and disposal at WIPP. Since TRU mixed waste will not be treated to LDR standards, treatment standards for the TRU mixed waste codes are not listed. The management of these waste streams is discussed in the SRS solid waste management strategy in Chapter 4, Section 4.1 of this volume.

Option analyses have been developed for two mixed low-level waste (MLLW) streams (SR-W025 and SR-W033). These streams will continue to be managed as TRU waste and used to blend without higher activity TRU waste.

The General Information Section also provides a brief description for each waste stream, which includes the following:

Volume: To facilitate future changes, waste stream volumes are not listed for the mixed wastes discussed in Chapter 3-5 in Volume II. Waste volume summary tables are found in Chapter 11, Volume II, of the STP.

Waste Stream Composition: Provides information about the physical form of the waste and serves as a major heading under which like streams are grouped.

Waste Characterization: Provides information on the chemical characterization of the waste stream and the confidence level of the information listed. The basis for waste characterization is either by sampling and analysis or by process knowledge. The confidence level for either method of waste characterization for the hazardous waste constituent is expressed as high, medium, or low.

A high-confidence level reflects detailed knowledge of the waste through extensive sampling and analysis, which may include regulatory prescribed tests such as TCLP, or by process knowledge, which is based on process specification or design, reliable mass balance calculation, or other controlled and accurate information.

A medium-confidence level is based on partial sampling and analysis or the use of test methods that do not provide the most accurate results. Medium process knowledge confidence is based on indirect or less controlled knowledge that enables conclusions to be drawn about contaminants in a waste, but with uncertainty concerning contaminant levels.

A low-confidence level indicates no sampling and analysis data or highly uncertain data due to chemical or radiological interference. A low-confidence level for process knowledge indicates a great amount of uncertainty about the characterization of the waste. Only a few SRS waste streams have a low confidence level. These streams are addressed in a conservative manner in the treatment option analysis performed in the STP.

Radiological Characterization: Describes the radiochemical nature of the waste. Radionuclides and activity levels are listed, if known. Wastes are contact handled unless specified to the contrary. Mixed low-level wastes are found in Chapter 3, mixed transuranic wastes are found in Chapter 4, and mixed high-level wastes are found in Chapter 5.

Waste Codes: Lists the RCRA waste code classification of the contaminants present in the waste. The use of an additional letter at the end of the RCRA code is a descriptor used by DOE to denote the particular LDR treatment subcategory that is applicable in cases where RCRA treatment standards list more than one treatment method or concentration standard depending on the wasteform. (See Table 1.3 of this section for further information.) 
LDR Treatment Standards: Provides treatment information from the RCRA regulations regarding LDR requirements for the waste stream. Explanation on the basis and regulatory background for the LDR treatment standards is found later in this chapter.

\section{Technology and Capacity Needs}

The second part of the discussion on each waste stream in Volume II deals with the treatment technology. A flow diagram of the process steps is provided. Justification is provided for how the treatment option meets the regulatory standard if an IDOA has been performed. Information is given on capacity requirements to treat the waste and what treatment facility needs must be met to facilitate treating the waste.

\section{Treatment Option Information}

This part discusses the type of treatment technology and other technical features regarding the identified treatment option. Information is provided on the operational and regulatory status of the treatment option. For onsite treatment options, a description of the action needed to bring the facility into operation is given if applicable. Discussion of offsite DOE facilities lists the facility status.

\section{Treatment Option Status and Uncertainties}

A status on the budget requirements for the treatment option and known external uncertainties of a budgetary, technical, or administrative nature are provided.

MLLW in Sections 3.2 and 3.3 of Chapter 3 are described with a slightly modified format than that described above. Section 3.2 addresses waste streams that do not have an identified technology and must undergo further technology development or request a treatability variance. Section 3.3 contains MLLW streams being managed as MTRU that require further waste characterization.

MTRU in Chapter 4 has a three-part description that includes General Information, Technology and Capacity Needs, Treatment Option Status, and Uncertainty Issues.

The description format for waste streams in Chapter 5 follows the same outline for the waste streams in Section 3.1 .

\section{Land Disposal Restrictions}

Each contaminant regulated by RCRA is given a waste code (for example, D008 or F006). The waste code either identifies the contaminant, the industrial process creating the waste, or both. For some of the waste codes, DOE has assigned a letter suffix to further identify a waste stream matrix (for example, D008A describes a waste hazardous for lead content, D008B describes hazardous waste lead in the form of lead/acid batteries, and D008C describes hazardous waste lead in the form of radioactive lead solids). (See Table 1.3, Chapter 1, Volume II.)

For each waste stream in Volume II, Land Disposal Restriction (LDR) data provide the concentration based treatment standard or range of standards or the specified technology required to be met by the LDR regulations. If the waste stream meets the LDR definition of debris, one of seventeen alternative debris technologies may be applied to meet the LDR regulations, or the waste may be treated to meet the waste specific treatment standard. These standards were developed for waste that is to be disposed of on the land (defined as landfills, surface impoundments, waste piles, injection wells, land treatment units, salt dome, or salt bed formations). The treatment standards, set by EPA, must be met before the waste can be land disposed. The standards are usually a concentration level for the waste based on Toxicity Characteristic Leaching Procedure (TCLP) test results or total composition analysis results. The standards vary based on whether the waste stream is a wastewater, which is water contaminated with less than $1 \%$ total organic carbon (<1\% TOC) and with less than $1 \%$ total suspended solids (<1\% TSS); or a nonwastewater, which is everything else. For F001-F005 listed wastes, the definition of wastewater is less than $1 \%$ by weight total organic carbon (<1\% TOC) for the solvent water mixture or the F001-F005 solvent constituent listed in 40 CFR Part 268.41. 
In September 1994, EPA issued the Phase II LDR rule that established a Universal Treatment Standard list (UTS) of concentration based standards for almost all hazardous characteristic and listed waste. Also, concentration based treatment standards based on UTS were established for the organic TC wastes (D018- D043), ten newly listed wastes, and D012-D014 pesticides. The new rule also required that UTS be met for any underlying hazardous constituent in wastes determined to be hazardous for waste codes D001, D002, and D012-D043. In April 1996, EPA issued the Phase III LDR Rule that established that generators would be required to treat the underlying hazardous constitutes in wastes determined to be hazardous for waste codes D001, D002, D003, and D012-D043 that were disposed in non-CWA facilities (land-based units) as well as CWA facilities. In March 1996, the President signed into law the Land Disposal Program Flexibility Act of 1996 which, among other things, negated the provision that generators with decharacterized wastewater managed in CWA equivalent facilities have to identify Underlying Hazardous Constituents and treat them to the Universal Treatment Standard. The Flexibility Act removed a large portion of the intent and strength of the finalized Phase III rule.

One of the issues that remained in the Phase III Rule was the formalization of EPA's Combustion Strategy. The Strategy provides guidance that combustion of certain inorganic, metal-bearing hazardous wastes is impermissible dilution under the LDR program. In order to be incinerated or substituted as fuel in a BIF, certain inorganic metal-bearing hazardous wastes would have to meet one of six criteria.

1. The waste must contain hazardous organic constitutes or cyanides at levels exceeding the constituent specific UTS.

2. The waste is an organic, debris-like material (wood, paper, plastic or cloth) that is contaminated with inorganic, metal-bearing hazardous waste.

3. The waste has a BTU value equal to or greater than $5,000 \mathrm{BTU} / \mathrm{lb}$.

4. The waste is co-generated with other wastes for which combustion is a specified treatment standard.

5. The waste is subject to a federal and/or state provision that requires a reduction of organics (including biological agents).

6. The waste contains $>1 \%$ TOC.

This combustion strategy will play an important role in what hazardous/mixed waste streams may use combustion as a method of treatment.

In a continuing effort to simplify LDR notification, certification, and recordkeeping requirements, EPA completely revamped §268.7 in the May 12, 1997, Phase IV, Part 1 rulemaking. Most significantly, a one-time LDR notice will suffice (after authorized states modify their programs) compared to previous requirements for a notice to accompany each shipment. In addition, the federal California list waste program was eliminated by this rule. The Phase IV, Part 2 rulemaking was finalized May 26, 1998. The most significant aspects of this rule include: (1) revision of UTS for 12 metal wastes; (2) modification of LDR treatment standards for the eight toxicity characteristic (TC) metals (D004-D011); and (3) promulgation of alternative LDR treatment standards for contaminated soils.

In determining the concentration based treatment standards, EPA has examined data from various treatment methods and determined which method is the best (and commercially available) for treating each waste code. That method has been identified as the Best Demonstrated Available Technology (BDAT). Wastes are not required to be treated by the BDAT. Any treatment method may be used, but where concentration based standards exist for a waste code, that standard must be met regardless of the treatment method employed. The BDAT is simply the treatment method that EPA examined and used in developing the concentration based treatment standards for the LDR program.

In some cases, the nature of the waste makes chemical analysis of a treated wasteform very difficult or unreliable. In these cases, EPA has required a treatment method called a specified technology to be performed before land disposal. When specified technologies are identified as the treatment standard for a particular waste code, that technology must be used to treat that waste (alternative treatments would only be allowed if a treatability variance were submitted and approved or regulatory discretions were granted).

In addition to setting those standards noted above, EPA also has recognized that these treatment standards were developed based upon determination of the BDAT for the "normal" waste stream matrices such as electroplating sludges, paint thinners, solvents, etc. EPA believes that treatment standards based on BDATs for 
these waste matrices are not appropriate for treating wastes with a significantly different physical form such as soil, rocks, equipment, plastic, etc. Therefore, EPA issued treatment standards specifically for debris (these regulations were published in the August 18, 1992 Federal Register) and has committed to issuing treatment standards specifically for soil (regulations still under development at EPA). Until such time as the new soil standards are issued, soils receiving treatment must meet the treatment standards promulgated for the "normal" waste streams as noted.

\section{Specified Technology Treatment Requirements}

The following are regulatory definitions regarding specific treatment technology requirements for particular waste streams from the LDR regulations. These are not all the definitions but are the ones used in listing treatment requirements for SRS mixed waste streams. These definitions are listed here as well as in Chapter 12 for ease of reference.

ADGAS-venting of compressed gases into an absorbing or reacting media (i.e., solid or liquid); venting can be accomplished through physical release utilizing valves/piping; physical penetration of the container, and penetration through detonation.

AMLGM-amalgamation of elemental mercury with inorganic reagents such as copper, zinc, nickel, gold, and sulfur that results in a nonliquid, semi-solid amalgam and thereby reduces potential emissions of elemental mercury vapors to the air.

CHOXD-chemical or electrolytic oxidation utilizing the following oxidation reagents (or waste reagents) or combinations of reagents: (1) hypochlorite (e.g., bleach); (2) chlorine; (3) chlorine dioxide; (4) ozone or UV (ultraviolet light) assisted ozone; (5) peroxides; (6) persulfates; (7) perchlorates; (8) permanganates; and/or (9) other oxidizing reagents of equivalent efficiency, performed in units operated such that a surrogate compound or indicator parameter has been substantially reduced in concentration in the residuals (e.g., total organic carbon can often be used as an indicator parameter for the oxidation of many organic constituents that cannot be directly analyzed in wastewater residues). Chemical oxidation specifically includes what is commonly referred to as alkaline chlorination.

CMBST-High-temperature organic destruction technologies, such as combustion in incinerators, boilers, or industrial furnaces operated in accordance with the applicable requirements of RCRA regulations Part 264, Subpart O, Part 265, Subpart O or Part 266, Subpart H, and in other units in accordance with applicable technical operating requirements. Certain noncombustive technologies, such as the Catalytic Extraction Process, also qualify.

DEACT-deactivation to remove the hazardous characteristic of a waste due to its ignitability, corrosivity, and/or reactivity.

HLVIT-vitrification of high-level mixed radioactive waste in units in compliance with all applicable radioactive protection requirements under control of the Nuclear Regulatory Commission.

IMERC-incineration of wastes containing organics and mercury in units operated in accordance with the technical operating requirements of 40 CFR Part 264 Subpart O and Part 265 Subpart O. All wastewater and nonwastewater residues derived from this process must then comply with the corresponding treatment standards per waste code with consideration of any applicable subcategories (e.g., High or Low Mercury Subcategory).

MACRO (Specified Technology Treatment Standard)-macroencapsulation with surface coating materials such as polymeric organics (e.g., resins and plastics) or with a jacket of inert inorganic materials to substantially reduce surface exposure to potential leaching media. Macroencapsulation specifically does not include any material that would be classified as a tank or container according to 40 CFR 260.10.

MACRO (alternative standard for debris)-identical definition to the one immediately above for the technology based standard except this definition excludes the last sentence referring to use of materials that could be classified as a tank or container. 
NEUTR-neutralization uses these chemicals either alone or in combination: (1) acids; (2) bases; or (3) water (including wastewaters) resulting in a $\mathrm{pH}$ greater than 2 but less than 12.5 as measured in the aqueous residuals.

RLEAD-thermal recovery of lead in secondary lead smelters.

RMERC-retorting or roasting in a thermal processing unit capable of volatilizing mercury and subsequently condensing the volatilized mercury for recovery. The retorting or roasting unit (or facility) must be subject to one or more of the following: (a) a National Emissions Standards for Hazardous Air Pollutants (NESHAP) for mercury; (b) a Best Available Control Technology (BACT) or a Lowest Achievable Emission Rate (LAER) standard for mercury imposed pursuant to a Prevention of Significant Deterioration (PSD) limit; or (c) a state permit that establishes emission limitations (within meaning of section 302 of the Clean Air Act) for mercury. All wastewater and nonwastewater residues derived from this process must then comply with the corresponding treatment standards per waste code with consideration of any applicable subcategories (e.g., High or Low Mercury Subcategory).

RMETL-recovery of metals or inorganics utilizing one or more of the following direct physical/removal technologies: (1) ion exchange; (2) resin or solid (i.e., zeolites) adsorption; (3) reverse osmosis; (4) chelation/solvent extraction; (5) freeze crystallization; (6) ultrafiltration; and/or (7) simple precipitation (i.e., crystallization). (Note: This does not preclude the use of other physical phase separation or concentration techniques such as decantation, filtration (including ultrafiltration), and centrifugation when used in conjunction with the above listed recovery technologies).

RORGS-recovery of organics utilizing one or more of the following technologies: (1) distillation; (2) thin film evaporation; (3) steam stripping; (4) carbon adsorption; (5) critical fluid extraction; (6) liquid-liquid extraction; (7) precipitation/crystallization (including freeze crystallization); or (8) chemical phase separation techniques (i.e., addition of acids, bases, demulsifiers, or similar chemicals): (Note: This does not preclude the use of other physical phase separation techniques such as decantation, filtration [including ultrafiltration], and centrifugation when used in conjunction with the above listed recovery techniques.)

RTHRM-thermal recovery of metals or inorganics from nonwastewaters in units identified as industrial furnaces according to 40 CFR 260.10 (1), (6), (7), (11), and (12) under the definition of "industrial furnaces".

STABL-Stabilization with the following reagents (or waste reagents) or combinations of reagents: (1) Portland cement; or (2) lime/pozzolans (e.g., fly ash and cement kiln dust). (Note: This does not preclude the addition of reagents [e.g., iron salts, silicates, and clays] designed to enhance the set/cure time and/or compressive strength, or to overall reduce the leachability of the metal or inorganic.)

\section{Permitting Strategy for Treatment Activities}

There are several options for locating and obtaining regulatory approval for RCRA treatment. A strategy for determining the appropriate and allowable option is important in developing costs and schedules for the implementation of treatment activities determined by the STP. A strategy is also important in determining and minimizing issues to be addressed in the compliance order pertaining to continued storage and future treatment of prohibited wastes. Treatment may occur in RCRA 90-day accumulation areas (also referred to as staging areas), RCRA interim status units, or RCRA permitted units. It must be ensured that certain conditions are met prior to selecting one of these options.

90-Day Accumulation Areas: A provision exists that allows generators who meet the requirements of SCHWMR R.61-79.262.34, to store and treat hazardous waste in a 90-day accumulation area (staging area) without having to obtain a RCRA permit or interim status. Treatment in a staging area must occur in tanks or containers or in a containment building. General design and operating standards must be met as well as specific standards as applicable for containers, tanks, and containment buildings. Waste must be removed from the staging area within 90 days. Specific notifications must be made in accordance with the requirements of the Land Disposal Restrictions for wastes that undergo treatment in a 90-day staging area. In addition, a Waste Analysis Plan may be necessary depending on the wastes and treatment to be performed in the staging area. 
It is advantageous to select the 90-day staging area provision as an option for treatment strategy. No regulatory approvals or permitting is necessary. This results in an accelerated schedule for treatment implementation and reduced costs due to the lack of any permitting activities.

However, several instances may exist where 90-day areas are not allowed as an option for treatment. Treatment must then occur in a RCRA interim status unit or a permitted unit. T his may occur in the following instances:

- Waste is currently in permitted storage.

- It may not be possible to complete treatment of waste in the accumulation area within the 90 days.

- Treatment will not occur in a tank, container, or containment building.

Interim Status Unit: Interim status is a relatively short-term mechanism that allows certain limited activities to be conducted while the associated unit awaits or undergoes a thorough review in the permitting process. A unit may operate for more than 90 days under interim status without a permit when certain conditions are met. A unit that currently operates under interim status may be allowed to add new treatment processes. New additional storage or treatment units may also be allowed to operate under interim status. Regulatory approval of changes in interim status units are based on several criteria such as being necessary to comply with federal, state, or local requirements, or a demonstrated lack of available treatment or storage capacity at the facility. To request interim status unit changes or additions, a revised Part A application must be filed along with a justification for the request based on required approval criteria.

Part A revision is a relatively uncomplicated task and can be accomplished with a minimal amount of time and expense. Regulatory review may be accomplished in moderate time frames. It is important to note that once interim status is granted for a facility a request for a full permit application, as discussed below, may be requested by the regulatory agencies at any time.

Part A revisions to add treatment processes or operate a new unit under interim status may not always be approved by the regulatory agency based on inadequate justification by the facility requesting the revision. In addition, it is not allowable to add interim status treatment processes to a unit that is already operating under a RCRA permit. In these cases where treatment processes may not gain interim status, a modification to the RCRA permit may be necessary to add treatment processes or operate a new unit.

Permitted Unit: A final option for obtaining regulatory approval for a treatment process is a RCRA permit modification. A permit is obtained by first revising Parts A and B of the RCRA permit application. As discussed, a revision to the Part A is a relatively uncomplicated process.

If a unit already operates under a RCRA permit, a revision to the Part B permit application will be necessary to add a new treatment process. The difficulty in preparing this type of revision is dependent on the complexity of the treatment activity. Generally this task is not difficult or costly.

If a unit does not already operate under a RCRA permit, a Part B application revision to add the new unit for treatment will be necessary. This is a complicated process requiring a detailed description of the design and operation of the unit and discussion on how the unit will comply with all applicable RCRA requirements. The preparation of this documentation is costly and time consuming.

Regulatory review times are dependent on the complexity of the application revisions. Reviews of modifications to existing units may take weeks while those for a new unit may take years. The review process may include the issuance of one or more Notices of Deficiency by the agencies requesting a revision to the application to add or clarify information. Once the regulatory agencies determine the modification to the permit application is complete, a draft and final permit modification is issued for the new treatment process or new treatment unit. This process is also determined by the complexity of the permit application modification.

Wastewater and Recycling: In addition to treatment in RCRA 90-day accumulation areas, interim status units, or permitted units, hazardous waste may be managed in a wastewater treatment facility or through recycle activities if certain conditions are met [SCHWMR R.61-79.264.1 (g) and R.61-79.265.1(c)]. 
Hazardous waste may be treated in an eligible wastewater treatment unit that is operated and discharged in accordance with the requirements of the South Carolina Pollution Control Act (PCA). The unit must also meet the regulatory definition of a tank. Eligible wastewater treatment units managing hazardous waste are subject to PCA performance standards and permitting requirements, but may not be subject to RCRA permitting requirements. However, there are LDR notification requirements under 40 CFR 268.7 and equivalent state hazardous waste management regulations for hazardous wastes that are managed in PCA facilities.

In some cases, treatment activities performed as a recycling operation would not be subject to RCRA permitting requirements. This exclusion is dependent on what the material is and how it is recycled.

\section{EPA Hazardous Waste Codes with Subcategories Defined under the LDR Program}

Table 1.2 lists EPA hazardous waste codes for which EPA has developed subcategories (40 CFR Sections 268.41 through 268.43, Tables CCWE, 2, 3, and CCW). For each subcategory, DOE has assigned a letter subcode. The subcategories represent unique LDR treatability groups with distinct treatment standards. In addition, DOE has assigned a subcategory (with subcode "X") for wastes that, because of a lack of characterization information, could not be put into an appropriate EPA defined subcategory. This table has been developed in support of the MWIR to provide explanation for the letter subcodes used in Chapters 3-5 of the STP, Volume II. The table may be subject to change. 
Table 1.2

\section{EPA Hazardous Waste Codes with Subcategories}

\begin{tabular}{|c|c|c|c|}
\hline $\begin{array}{l}\text { EPA } \\
\text { Code }\end{array}$ & $\begin{array}{c}\text { Sub } \\
\text { Code }\end{array}$ & Subcategory & Description \\
\hline \multirow[t]{3}{*}{ D001 } & A & $\begin{array}{l}\text { Ignitable liquids high TOC } \\
\text { nonwastewaters }\end{array}$ & $\begin{array}{l}\text { Ignitable liquids as defined in } 40 \text { CFR } 261.21 \text { containing } \\
10 \% \text { or greater Total Organic Carbon (TOC) }\end{array}$ \\
\hline & B & Ignitable liquids, wastewaters & $\begin{array}{l}\text { Ignitable wastes as identified in } 40 \text { CFR } 261.21 \text { managed } \\
\text { as wastewater [e.g., in Clean Water Act surface } \\
\text { impoundments or land disposal units (or their equivalent); } \\
\text { or in Safe Drinking Water Act underground injection } \\
\text { wells] }\end{array}$ \\
\hline & $\mathrm{C}$ & $\begin{array}{l}\text { Ignitable waste, low TOC } \\
\text { nonwastewaters }\end{array}$ & $\begin{array}{l}\text { All other ignitable waste as identified in } 40 \text { CFR } 261.21 \\
\text { that is neither a high TOC nor managed as wastewater }\end{array}$ \\
\hline \multirow[t]{2}{*}{ D002 } & $\bar{A}$ & $\begin{array}{l}\text { Corrosive wastewater-acid, alkaline } \\
\text { or other }\end{array}$ & $\begin{array}{l}\text { Corrosive waste, as identified in } 40 \text { CFR 261.22, } \\
\text { managed as wastewater. [e.g., in Clean Water Act surface } \\
\text { impoundments or land disposed units (or their } \\
\text { equivalent); or in Safe Drinking Water Act underground } \\
\text { injection wells] }\end{array}$ \\
\hline & $\mathrm{B}$ & $\begin{array}{l}\text { Corrosive nonwastewater-acid, } \\
\text { alkaline or other }\end{array}$ & $\begin{array}{l}\text { Corrosive waste, as identified in } 40 \text { CFR } 261.22 \text {, not } \\
\text { managed as wastewater }\end{array}$ \\
\hline \multirow[t]{5}{*}{ D003 } & A & Reactive cyanides & $\begin{array}{l}\text { Cyanide-bearing wastes that, when exposed to } \mathrm{pH} \\
\text { conditions between } 2 \text { and } 12.5 \text {, generate hazardous } \\
\text { quantities of toxic gases }\end{array}$ \\
\hline & B & Reactive sulfides & $\begin{array}{l}\text { Sulfide-bearing wastes that, when exposed to } \mathrm{pH} \\
\text { conditions between } 2 \text { and } 12.5 \text {, generate hazardous } \\
\text { quantities of toxic gases }\end{array}$ \\
\hline & $\mathrm{C}$ & Explosives & $\begin{array}{l}\text { Waste capable of detonation or explosive reaction under } \\
\text { various conditions, or is a forbidden Class A or Class B } \\
\text { explosive under DOT regulations }\end{array}$ \\
\hline & $\mathrm{D}$ & Water reactives & $\begin{array}{l}\text { Waste, as defined in } 40 \text { CFR 261.23(a)(2), (3), or (4), that } \\
\text { is either very reactive with water, or is capable of } \\
\text { generating toxic or explosive gases with water }\end{array}$ \\
\hline & $\mathrm{E}$ & Other reactives & $\begin{array}{l}\text { Reactive waste that, per } 40 \text { CFR } 261.23(\mathrm{a})(1) \text {, is } \\
\text { normally unstable and readily undergoes violent change } \\
\text { without detonating }\end{array}$ \\
\hline \multirow[t]{2}{*}{ D006 } & A & TCLP toxic for cadmium & $\begin{array}{l}\text { Those wastes that exhibit the toxicity characteristic for } \\
\text { cadmium }\end{array}$ \\
\hline & $\bar{B}$ & Cadmium-containing batteries & $\begin{array}{l}\text { Batteries containing leachable levels of cadmium above } \\
1.0 \mathrm{mg} / \text { liter }\end{array}$ \\
\hline \multirow[t]{3}{*}{ D008 } & A & TCLP toxic for lead & $\begin{array}{l}\text { Those wastes that exhibit the toxicity characteristic for } \\
\text { lead }\end{array}$ \\
\hline & B & Lead acid batteries & $\begin{array}{l}\text { Lead acid batteries that are identified as RCRA } \\
\text { hazardous wastes and which are not excluded from } \\
\text { regulation under the land disposal restrictions }\end{array}$ \\
\hline & $\mathrm{C}$ & Radioactive lead solids & $\begin{array}{l}\text { Lead solids, including elemental forms of lead, but not } \\
\text { including treatment residuals that can be stabilized or } \\
\text { organo-lead materials that can be incinerated (then } \\
\text { stabilized as ash) }\end{array}$ \\
\hline \multirow[t]{2}{*}{ D009 } & A & TCLP toxic for mercury & $\begin{array}{l}\text { Nonwastewaters that exhibit the toxicity characteristic } \\
\text { for mercury and contain less than } 260 \mathrm{mg} / \mathrm{kg} \text { total } \\
\text { mercury }\end{array}$ \\
\hline & B & High mercury (contains organics) & $\begin{array}{l}\text { Nonwastewaters that exhibit the toxicity characteristic } \\
\text { for mercury, contain greater than or equal to } 260 \mathrm{mg} / \mathrm{kg} \\
\text { total mercury, also contain organics, and are not } \\
\text { incinerator residues. }\end{array}$ \\
\hline
\end{tabular}


Table 1.2

EPA Hazardous Waste Codes with Subcategories (cont'd)

\begin{tabular}{|c|c|c|c|}
\hline $\begin{array}{l}\text { EPA } \\
\text { code }\end{array}$ & $\begin{array}{c}\text { Sub } \\
\text { Code }\end{array}$ & Subcategory & Description \\
\hline & $\mathrm{C}$ & High mercury (contains inorganics) & $\begin{array}{l}\text { Nonwastewaters that exhibit the toxicity characteristic } \\
\text { for mercury, contain greater than or equal to } 260 \mathrm{mg} / \mathrm{kg} \\
\text { total mercury, are inorganic, and may include } \\
\text { incinerator residues and residues from mercury roasting } \\
\text { and retorting (RMERC) operations }\end{array}$ \\
\hline & $\mathrm{D}$ & $\begin{array}{l}\text { Elemental mercury contaminated } \\
\text { with radioactive materials }\end{array}$ & $\begin{array}{l}\text { Elemental mercury contaminated with radioactive } \\
\text { materials }\end{array}$ \\
\hline & $\mathrm{E}$ & $\begin{array}{l}\text { Hydraulic oil contaminated with } \\
\text { mercury and radioactive materials }\end{array}$ & $\begin{array}{l}\text { Hydraulic oil exhibiting the toxicity characteristic for } \\
\text { mercury and which is contaminated with radioactive } \\
\text { materials }\end{array}$ \\
\hline & $\mathrm{F}$ & Mercury wastewaters & All D009 waste managed as wastewater \\
\hline \multirow[t]{2}{*}{ F003 } & A & Spent nonhalogenated solvents & $\begin{array}{l}\text { F003 solvent due to the presence of one of the } \\
\text { following: acetone, ethyl acetate, ethyl benzene, ethyl } \\
\text { ether, methyl isobutyl ketone, n-butyl alcohol, and } \\
\text { xylene. Also cyclohexane, but only if F001-F005 } \\
\text { solvents other than methanol and/or carbon disulfide } \\
\text { (F005) are also present. Also methanol, but only if } \\
\text { F001-F005 solvents other than cyclohexane and/or } \\
\text { carbon disulfide (F005) are also present. }\end{array}$ \\
\hline & B & $\begin{array}{l}\text { Cyclohexane/methanol/carbon } \\
\text { disulfide only }\end{array}$ & $\begin{array}{l}\text { F003 solvent due to the presence of cyclohexane, } \\
\text { methanol or carbon disulfide, but only if no other F001- } \\
\text { F005 solvents are present (except cyclohexane, } \\
\text { methanol and/or carbon disulfide are also present) }\end{array}$ \\
\hline \multirow[t]{4}{*}{ F005 } & A & Spent nonhalogenated solvents & $\begin{array}{l}\text { The following spent non-halogenated solvents: } \\
\text { benzene, isobutanol, methyl ethyl ketone, pyridine, and } \\
\text { toluene. Also, carbon disulfide if F001-F005 solvents } \\
\text { other than cyclohexane (F003) and/or methanol (F003) } \\
\text { are also present. Also, 2-ethoxyethanol and } 2 \\
\text { nitropropane, but only if other F001-F005 solvents are } \\
\text { also present. }\end{array}$ \\
\hline & B & $\begin{array}{l}\text { Solvent waste listed for } \\
\text { 2-nitropropane only }\end{array}$ & $\begin{array}{l}\text { Waste containing 2-nitropropane as the only F001-F005 } \\
\text { listed solvent }\end{array}$ \\
\hline & $\mathrm{C}$ & $\begin{array}{l}\text { Solvent waste listed for } \\
\text { 2-ethoxyethanol only }\end{array}$ & $\begin{array}{l}\text { Waste containing 2-ethoxyethanol as the only F001- } \\
\text { F005 listed solvent }\end{array}$ \\
\hline & $\mathrm{D}$ & $\begin{array}{l}\text { Cyclohexane/methanol/ } \\
\text { carbon disulfide only }\end{array}$ & $\begin{array}{l}\text { F005 listed mixed waste for which the specific F005 } \\
\text { constituent is not identified. F005 solvent due to the } \\
\text { presence of carbon disulfide, but only if no other F001- } \\
\text { F005 solvents are present, except that cyclohexane } \\
\text { (F003) and/or methanol (F003) may also be present. }\end{array}$ \\
\hline F025 & $\bar{A}$ & F025 light ends & $\begin{array}{l}\text { Light ends listed for one or more of the following: carbon } \\
\text { tetrachloride; chloroform; } 1,2 \text {-Dichloroethane; } 1,1 \text { - } \\
\text { Dichloroethylene; Methylene chloride; } 1,1,2 \\
\text { Trichloroethane; Trichloroethane or vinyl chloride; plus } \\
\text { wastes qualifying as F025 light ends, but characterization } \\
\text { information is insufficient to determine specific } \\
\text { contaminants. }\end{array}$ \\
\hline F025 & B & Spent filter/aids and desiccants & $\begin{array}{l}\text { Spent filters/aids containing one or more of the } \\
\text { following: Carbon tetrachloride, chloroform, methylene } \\
\text { chloride, 1, 1, 2-Trichloroethane, Trichloroethylene, } \\
\text { vinyl chloride, hexachlorobutadiene, or } \\
\text { hexachloroethane, plus wastes qualifying as F025 spent } \\
\text { filters/aids or desiccants, but characterization is } \\
\text { insufficient to determine specific contaminants. }\end{array}$ \\
\hline
\end{tabular}


Table 1.2

EPA Hazardous Waste Codes with Subcategories (cont'd)

\begin{tabular}{|c|c|c|c|}
\hline $\begin{array}{l}\text { EPA } \\
\text { Code }\end{array}$ & $\begin{array}{c}\text { Sub } \\
\text { Code }\end{array}$ & Subcategory & Description \\
\hline \multirow[t]{2}{*}{ P047 } & $\mathrm{A}$ & 4, 6-dinitro-o-cresol & $\begin{array}{l}\text { 4,6-Dinitro-o-cresol as a discarded commercial chemical } \\
\text { product, off-specification species, container residue, or } \\
\text { spill residue. }\end{array}$ \\
\hline & B & 4, 6-dinitro-o-cresol salts & $\begin{array}{l}\text { 4, 6-Dinitro-o-cresol salts as discarded commercial } \\
\text { chemical products, off-specification species, container } \\
\text { residues, or spill residues. }\end{array}$ \\
\hline \multirow[t]{2}{*}{ P059 } & $\mathrm{A}$ & Heptachlor & $\begin{array}{l}\text { Heptachlor as a discarded commercial chemical product, } \\
\text { off-specification species, container residue, or spill } \\
\text { residue. }\end{array}$ \\
\hline & $\mathrm{B}$ & Heptachlor epoxide & $\begin{array}{l}\text { Heptachlor epoxide as a discarded commercial chemical } \\
\text { product, off-specification species, container residue, or } \\
\text { spill residue. }\end{array}$ \\
\hline \multirow[t]{3}{*}{ P065 } & $\mathrm{A}$ & $\begin{array}{l}\text { Mercury fulminate-high mercury } \\
\text { incinerator or RMERC residues }\end{array}$ & $\begin{array}{l}\text { Nonwastewaters with greater than or equal to } 260 \mathrm{mg} / \mathrm{kg} \\
\text { total mercury and that are residues from either thermal } \\
\text { treatment or mercury roasting or retorting (RMERC) of } \\
\text { wastes containing mercury fulminate. }\end{array}$ \\
\hline & B & $\begin{array}{l}\text { Mercury fulminate waste (not from } \\
\text { thermal treatment or RMERC) }\end{array}$ & $\begin{array}{l}\text { Nonwastewater mercury fulminate waste, regardless of } \\
\text { mercury content that is neither residues from thermal } \\
\text { treatment nor residues from RMERC. }\end{array}$ \\
\hline & $\mathrm{C}$ & $\begin{array}{l}\text { Mercury fulminate - low mercury } \\
\text { RMERC residues }\end{array}$ & $\begin{array}{l}\text { Nonwastewaters with less than } 260 \mathrm{mg} / \mathrm{kg} \text { total mercury } \\
\text { and that are residues from RMERC of wastes containing } \\
\text { mercury fulminate. }\end{array}$ \\
\hline \multirow[t]{2}{*}{ P065 } & $\mathrm{D}$ & $\begin{array}{l}\text { Mercury fulminate - low mercury } \\
\text { incinerator residues (not RMERC) }\end{array}$ & $\begin{array}{l}\text { Nonwastewaters with less than } 260 \mathrm{mg} / \mathrm{kg} \text { total mercury } \\
\text { and that are residues from RMERC of wastes containing } \\
\text { mercury fulminate. }\end{array}$ \\
\hline & $\mathrm{E}$ & Mercury fulminate wastewaters & $\begin{array}{l}\text { All P065 (mercury fulminate) waste managed as } \\
\text { wastewaters. }\end{array}$ \\
\hline \multirow[t]{5}{*}{ P092 } & $\mathrm{A}$ & $\begin{array}{l}\text { Phenyl mercury acetate } \\
\text { nonwastewater } \\
\text { high mercury incinerator or RMERC } \\
\text { residues }\end{array}$ & $\begin{array}{l}\text { Nonwastewater phenyl mercury acetate wastes, } \\
\text { regardless of mercury content, that are residues from } \\
\text { either thermal treatment or mercury roasting or retorting } \\
\text { (RMERC) of wastes containing phenyl mercury acetate. }\end{array}$ \\
\hline & B & $\begin{array}{l}\text { Phenyl mercury acetate } \\
\text { nonwastewater } \\
\text { phenyl mercury acetate waste (not } \\
\text { from thermal treatment or RMERC) }\end{array}$ & $\begin{array}{l}\text { Nonwastewater phenyl mercury acetate wastes, } \\
\text { regardless of mercury content, that are not residues from } \\
\text { thermal treatment or residues from RMERC. }\end{array}$ \\
\hline & $\mathrm{C}$ & $\begin{array}{l}\text { Phenyl mercury acetate } \\
\text { nonwastewater } \\
\text { low mercury RMERC residues }\end{array}$ & $\begin{array}{l}\text { Nonwastewaters with less than } 260 \mathrm{mg} / \mathrm{kg} \text { total mercury } \\
\text { and that are residues from RMERC of wastes containing } \\
\text { phenyl mercury acetate. }\end{array}$ \\
\hline & $\overline{\mathrm{D}}$ & $\begin{array}{l}\text { Phenyl mercury acetate } \\
\text { nonwastewaters } \\
\text { low mercury incinerator residues (not } \\
\text { RMERC) }\end{array}$ & $\begin{array}{l}\text { Nonwastewater with less than } 260 \mathrm{mg} / \mathrm{kg} \text { total mercury } \\
\text { and that are residues from thermal treatment, but not } \\
\text { RMERC, of waste containing phenyl mercury acetate. }\end{array}$ \\
\hline & $\mathrm{E}$ & Phenyl mercury acetate wastewaters & $\begin{array}{l}\text { All P092 (mercury fulminate) waste managed as } \\
\text { wastewaters. }\end{array}$ \\
\hline \multirow[t]{4}{*}{ U151 } & A & High mercury nonwastewater & $\begin{array}{l}\text { Nonwastewaters with greater than or equal to } 260 \mathrm{mg} / \mathrm{kg} \\
\text { total mercury [including residues from mercury roasting } \\
\text { or retorting (RMERC) of U151 waste if it contains } \\
\text { greater than or equal to } 260 \mathrm{mg} / \mathrm{kg} \text { total mercury]. }\end{array}$ \\
\hline & $\mathrm{B}$ & $\begin{array}{l}\text { Low mercury nonwastewaters from } \\
\text { RMERC }\end{array}$ & $\begin{array}{l}\text { Nonwastewaters with less than } 260 \mathrm{mg} / \mathrm{kg} \text { total mercury } \\
\text { and that are residues from RMERC of U151 wastes }\end{array}$ \\
\hline & $\mathrm{C}$ & Low mercury nonwastewaters & $\begin{array}{l}\text { Non wastewaters with less than } 260 \mathrm{mg} / \mathrm{kg} \text { total mercury } \\
\text { that are not residues from RMERC }\end{array}$ \\
\hline & $\mathrm{D}$ & $\begin{array}{l}\text { Elemental mercury contaminated } \\
\text { with radioactive materials }\end{array}$ & \\
\hline
\end{tabular}


Table 1.2

EPA Hazardous Waste Codes with Subcategories (cont'd)

\begin{tabular}{|c|c|l|l|}
\hline $\begin{array}{c}\text { EPA } \\
\text { Code }\end{array}$ & $\begin{array}{c}\text { Sub } \\
\text { Code }\end{array}$ & \multicolumn{1}{|c|}{ Subcategory } & \multicolumn{1}{c|}{ Description } \\
\hline U240 & A & $\begin{array}{l}\text { 2, 4-D (aka dichlorophenoxyacetic } \\
\text { acid) }\end{array}$ & $\begin{array}{l}\text { 2, 4-D as a discarded commercial chemical product, off- } \\
\text { specification species, container residues, or spill residues. }\end{array}$ \\
\cline { 2 - 4 } & B & $\begin{array}{l}\text { 2,4-D (dichlorophenoxyacetic acids) } \\
\text { salts \& esters) }\end{array}$ & $\begin{array}{l}\text { 2, 4-D salts or esters as discarded commercial chemical } \\
\text { products, off-specification species, container residues, or } \\
\text { spill residues }\end{array}$ \\
\cline { 2 - 4 } & $\mathrm{C}$ & Unspecified U240 waste & $\begin{array}{l}\text { U240 waste, but characterization information is } \\
\text { insufficient to determine whether the A or B subcode is } \\
\text { appropriate. }\end{array}$ \\
\hline
\end{tabular}




\section{Chapter 2. Methodology}

This chapter discusses the methodology for preparing the STP, including the assumptions used, the treatment option selection process, the coordination with regulatory agencies and other stakeholders, the process of mixed waste characterization, and waste minimization/pollution prevention (WMin/PP).

\subsection{Assumptions Used for Preparation of Initial STPs}

The assumptions listed below were in effect at the time of approval of the STP on September 29, 1995. However, changes in conditions affecting the STP have occurred since approval. For example, with the signing of the Defense Authorization Bill on September 22, 1996, the No-Migration Petition requirement for the operation of WIPP was eliminated and an EPA certificate of compliance was added in place. Several treatment facilities, such as M-Area and CIF, have discontinued operations.

All sites used the following assumptions to provide a degree of consistency when preparing the STP. The assumptions were developed as a part of the "Draft Site Treatment Plan Development Framework" and reflect review and comment from the states and EPA.

- HLW will continue to be managed according to current plans at each site (i.e., Hanford, West Valley, SRS, Idaho National Engineering and Environmental Laboratory [INEEL]). Primarily due to potential safety concerns, HLW will not be transported offsite except as a treated, stable waste ready for disposal. The STP will not change management strategies for HLW.

- Regarding defense-related TRU waste, the STPs reflect DOE's current strategy on the Waste Isolation Pilot Plant (WIPP) opening and receiving a No-Migration Variance (NMV). An NMV is approved if the disposal facility can be shown to protect the environment. Wastes disposed in such a unit are not required to meet the LDR treatment standards. The STPs identify characterization, processing, and treatment of TRU waste to meet the WIPP Waste Acceptance Criteria (WAC). Consistent with this policy, treatment of mixed TRU waste to meet LDR standards will not be included in the STP.

- The STPs will recognize that DOE's policy regarding WIPP is under review and may change in the future. The STPs provide the flexibility to modify activities and milestones regarding TRU waste to reflect potential future changes in DOE policy.

Under current DOE policy, nondefense related TRU waste would not be disposed at WIPP. STPs should reflect LDR treatment of nondefense mixed TRU waste.

- DOE recognizes some states' preference for treatment of all wastes onsite. Where appropriate, existing onsite capacity will be used before new facilities are constructed. When onsite treatment or use of commercial or mobile facilities is not feasible, the use of existing offsite capacity, as well as the construction of new facilities, will be considered.

- Sites in the same state will investigate the practicality of consolidating treatment facilities.

- Mixed waste resulting from Soil and Grounds Closure Projects (SGCP) and decontamination and demolition (D\&D) activities will be factored into planning activities and equity discussions, particularly where using facilities in the STP are being considered for managing SGCP and D\&D mixed waste streams.

- On a volume basis, most of DOE's mixed wastes are to be treated onsite. Because of transportation concerns and costs, this includes process wastewater and some explosives and remotely handled waste. In addition, other large volume waste streams generally will be treated onsite. At a minimum, Richland (RL), Oak Ridge (OR), Idaho (ID), and Savannah River (SR) are to have onsite facilities to treat the majority of their wastes.

- The Programmatic Environmental Impact Statement (PEIS) is being performed in parallel with the development of the STPs. The STP process will provide information to the PEIS. Each site will prepare any necessary specific National Environmental Policy Act (NEPA) documentation before proceeding with a given project or facility required by the state or EPA as a result of the STP process.

- In support of DOE's "cradle to grave" waste management philosophy, disposal site location and criteria will be factored into state equity discussions, waste treatment facility designs, and the characteristics of the final wasteforms. 
In addition to the general DOE complexwide assumptions, SRS developed site-specific assumptions for use in developing the STP.

- To the extent possible, all mixed waste streams will have a preferred treatment option identified and/or option analysis complete in the STP. Those waste streams without a preferred treatment option will have a schedule for the development of the preferred option.

- $\quad$ All Savannah River Site high-level waste will be treated onsite.

- $\quad$ ER, Transition, and D\&D waste streams will be addressed in the STP to the extent that they are known. The STP does not address corrective action or remedial action pursuant to RCRA, Hazardous and Solid Waste Amendments, or CERCLA that do not involve the land disposal of hazardous waste (e.g., the placement of remediation wastes into or within a corrective action management unit). Corrective action or remedial action issues shall be addressed by the CERCLA Section 120 Federal Facility Agreement (FFA) effective August 16, 1993, and any hazardous waste permits issued or to be issued by the State of South Carolina and EPA or other actions under CERCLA. Methodology for modifying the STP for new ER, Transition, and D\&D waste streams will be incorporated into the text of the document. Investigation Derived Waste (IDW) will be managed per the IDW Management Plan as agreed by SCDHEC, EPA - Region IV, and SRS.

- If existing onsite treatment capacity is available for a particular waste stream, no further analysis will be performed for that waste with the exception of waste streams going to the CIF. To be responsive to stakeholders, alternatives to thermal treatment were addressed. Existing mixed waste treatment facilities are those facilities at SRS that are either presently operating or under construction (i.e., having been issued regulatory operating or construction permits). Existing mixed waste treatment facilities at the Savannah River Site include Savannah River Laboratory High Activity and Low Activity Treatment Tanks, M-Area Liquid ETF, F-Area and H-Area ETF, Z-Area Processing Facility, DWPF, M-Area Vendor Treatment Facility, and CIF. Existing non-RCRA disposal facilities include the E-Area Vaults and the Z-Area Saltstone Disposal Vaults.

- $\quad$ Since permits had not yet been issued for the M-Area Vendor Treatment Facility at the time of STP development, the Facility was referred to as a "new facility". However, treatment options analyses were not performed in the DSTP for the six original streams that served as a design basis for treatment by the M-Area Vendor Treatment Facility. Options analysis was conducted before the site treatment plan preparation and resulted in the selection of this treatment process that produces a superior wasteform. Options analyses for other SRS waste streams for which this technology is appropriate treatment have been done.

- Treatment schemes such as treatment in containers or containment buildings, privatization, mobile treatment, and others have been and will be investigated.

- $\quad$ The STP did not address moratorium waste in the preferred option analysis process.

- $\quad$ The level of detail for option analysis will vary in the STP from waste stream to waste stream.

- In all relevant STP flow diagrams, after the waste has been removed from the containers, the containers will be considered "empty" according to R61-79.261.7 of South Carolina Hazardous Waste Management Regulations (SCHWMR), thus requiring no treatment.

\subsection{Treatment Options Selection Process}

Because the STPs were prepared by the sites using a "bottom-up" approach, the resulting treatment configuration, when viewed from a national level, contained many redundancies and inefficiencies. The DSTP option selection process and methodology are explained in the Volume II, Sections 2.2.1, 2.2.2, and 2.2.3. As development of the STPs continued, an assessment was performed to determine what accommodations were necessary to blend the initial "bottom-up" approach into a more sensible national configuration of treatment systems as STP development was finalized. To facilitate this assessment, DOE established the Options Analysis Team (OAT) comprised of site representatives and members of the Headquarters' FFCAct Task Force. The OAT coordinated their efforts with the states through the National Governors Association (NGA) to ensure the national mixed waste configuration reflects both the states and DOE's concerns. As part of this evaluation, the impacts of implementing the emerging STP configuration, as well as alternative configurations, were evaluated. 
The focus of the OAT's efforts was on mixed low-level waste (MLLW). While the FFCAct also covers high-level waste (HLW) and mixed transuranic waste (MTRU), the strategies for managing these wastes have already been established. However, DOE recognized that modifications of these strategies might be needed as the programs evolve, and new information becomes available.

Changes to the baseline STP configuration proposed by the OAT were based on the following analyses:

1. Review of the STP baseline configuration to identify redundant and technically inefficient proposed treatment options.

2. Identification of alternative treatment configurations that emphasize key state and DOE concerns.

3. Evaluation of the STP baseline and alternate configurations against key evaluation areas to determine what combination of treatment options results in a configuration that best meets DOE's, the states', EPA's, and other stakeholders' concerns.

The results of the initial OAT analysis were shared with each of the sites and the state regulators, as well as DOE management. The OAT worked for several more months responding to state requests for additional analysis, incorporating ongoing site analysis, and responding to comments. The resulting configuration, as presented in the final development of the PSTPs, was DOE's best attempt to balance competing DOE and stakeholder interests.

As Site Treatment Plans throughout the DOE complex are approved, DOE created five focus groups to carry on the work of the OAT and provide oversight not only for development and implementation of treatment processes but also for disposal of treatment residuals. These focus groups addressed a broad range of mixed, hazardous, and low radioactive waste treatment and disposal concerns. The focus groups were Landfills, Groundwater, Mixed Waste, Tanks, and D\&D.

\subsubsection{Preferred Option (PO) Selection Process}

DOE-HQ prepared several guidance documents to assist the sites in working through treatment identification and selection of preferred options. Guidance is found in these documents:

- U. S. Department of Energy, Annotated Outline for the Draft Site Treatment Plans, Rev. 3 - draft, March 28, 1994

- U. S. Department of Energy, DSTP Development Framework Implementation Guidance, Revision 0, February 15, 1994

- U. S. Department of Energy, Draft Site Treatment Plan Cost Guidance, Revision 1, April 28, 1994

- U. S. Department of Energy, Draft Site Treatment Plan Development Framework, Revision 7, April 7, 1994

- U. S. Department of Energy, Guidance for Draft Site Treatment Plan (DSTP) Development, Rev. 4, May 10, 1994

- U. S. Department of Energy, Guidance for Preparation of DSTP, Appendix A, Revision 1, April 7, 1994

- U. S. Department of Energy, Protocol for Identifying a Potential Offsite Mixed Waste Treatment Option in the DSTP, Revision 1, March 7, 1994

- U. S. Department of Energy, Treatment Selection Guides, Revision 0, March 14, 1994

The Treatment Selection Guides provide information on selecting among treatment options by comparing the options on fundamental criteria such as regulatory compliance, environmental health and safety, treatment effectiveness, implementability, stakeholder concerns, life-cycle costs, and technology development. The DSTP Cost Information Guidance provides a level of consistency in the cost information by providing common cost assumptions. Drafts of these and other technical assistance documents were provided to the states and their comments incorporated into the final revision. These documents are available for review.

SRS technical personnel developed a method for selecting one preferred treatment process for each waste from a wide variety of treatment options. The SRS approach to treatment option analysis combined methods stipulated in the guidance provided by DOE (see above) with technology assessment techniques developed by WSRC. The detailed description of the treatment selection process appears in Sections 2.2.2 and 2.2.3. This process was completed for waste streams described in the PSTP. However, additional waste streams identified 
since the preparation of the PSTP required a technical option analysis for inclusion in the STP. As a result, it is appropriate to retain this section for the STP. Further justification for including this section is so that readers who are not familiar with previous developments to the STP can understand preferred treatment options listed in the approved STP.

\section{Options Evaluation Process}

This section contains two subsections. Subsection 2.2.2 contains an overview of the three-step process used to identify preferred options (POs). Subsection 2.2.3 contains detailed descriptions of each process step.

\subsubsection{Process Methodology Overview}

This section describes step by step the evaluation process used to determine preferred options (POs) for waste treatment.

\section{Step 1 Identify Feasible Options}

\section{Purpose}

To identify existing treatment facilities, existing production facilities with waste treatment capabilities, and planned treatment facilities that are technically feasible options for treating the SRS mixed waste streams.

It was assumed that facility modifications, permit modifications, etc., would be achievable.

\section{Performed by}

Technical personnel from each treatment and processing facility, along with the engineers and scientists assigned to the technical group who developed the STP.

\section{Step 2 Perform Initial Screening}

\section{Purpose}

To reduce the number of feasible options by assessing the technology success of the option.

The technology success assessment addresses the maturity and complexity of a feasible option to determine "viable" treatment options.

By assigning a Technology Success Factor (TSF) score to each feasible option, the feasible options are ranked. Those feasible options that received a high score become viable options requiring further analysis. Those feasible options that received a low score were rejected.

\section{Performed by}

Technical personnel from each treatment and processing facility, along with the engineers and scientists assigned to the technical group (IDOA), who developed the STP will perform the initial screening.

\section{Step 3 Perform In-Depth Options Analysis (IDOA)}

\section{Purpose}

The purpose of the IDOA is to identify the PO for each waste stream.

\section{Performed by}

Technical personnel from each treatment and processing facility, along with the engineers and scientists assigned to the technical group who developed the STP will perform the IDOA process. 


\subsubsection{Process Methodology Detailed Explanation}

For those mixed low-level waste streams requiring In-Depth Options Analysis (IDOA) to determine the preferred treatment option, the in-depth analysis considered five types of treatment:

- Existing onsite treatment facilities (e.g., F-Area and H-Area ETF)

- Existing production facilities with some potential capability to treat waste, or available floor space that could be refurbished to accommodate installation of treatment processes under the "Containment Building" provision of 40 CFR 265 and SCHWMR

- Planned treatment facilities

- Vendor processes operated either onsite or at the vendor's facility

- Waste treatment processing available from other DOE sites

\section{Initial Screening}

\section{Technology Risk Assessment and Technology Success Factor}

A methodology for assessing technology risk of a process or facility based upon Risk Management Concepts and Guidance written by the Analytical Sciences Corporation for the Defense Systems Management College was used. The methodology was originally developed by the Department of Defense (DOD) to assist with evaluation of new weapons systems.

The "risk" assessed in a technology risk assessment is the possibility that a process under consideration may be too new and too complex to perform as required. This type of assessment is biased in favor of simple and well established technology. According to the WSRC Conduct of Engineering Manual E7, Procedure 2.16, "Technology Risk Assessment," some questions to help determine technology risk indicators include:

- Are state-of-the-art advances in technology being used in the design?

- Is the equipment exposed to a harsh or unique environment?

- Does the design require complex integration of control systems or computer software?

- Is the design based on research and development or does it use mathematical models for prediction?

- Is the cost of recovery from system failure high?

- Is the design evolving as construction is going on?

- Is the design new or an extension of successful existing designs?

- Are familiar components being used in new, non-standard ways?

- Does the facility or process stand alone or must it interface with other facilities or processes?

Technology risk assessment does not determine whether the process or system is safe. Special analyses done in the design phase of a project ensure that new processes pose no hazard to workers, the public, or the environment.

No process or facility can be simpler than its most complex part or more mature than its newest part. Thus, a technology risk assessment begins with an examination of the whole process or facility to identify the part that has the most complex and the least mature technology. While the interaction of numerous parts and features may result in an overall process that is more complex and novel than its individual pieces, the identification of the crucial part is the first step in assessing the probability of a process or system failure.

The Maturity Factor (Pm) and the Complexity Factor $(\mathrm{Pc})$ are assigned "magnitudes," based on guidance in Table 2.1. When engineering assessment indicates the factors fall between the extremes noted, other magnitudes can be assigned. The Maturity and Complexity Factors are averaged to give the probability of failure $(\mathrm{Pf}) .(\mathrm{Pm}+\mathrm{Pc}) / 2=\mathrm{Pf}$. 
Table 2.1 - Probability of Failure

\begin{tabular}{|c|c|c|}
\hline Magnitude & Maturity Factor (Pm) & Complexity Factor (Pc) \\
\hline 0.1 & $\begin{array}{l}\text { - } \text { Components exist. } \\
\text { - } \text { Performance requirements are specific. } \\
\text { ranging is not bassumptions. }\end{array}$ & $\begin{array}{l}\text { - Design is simple. } \\
\text { - Design is complete before installation } \\
\text { begins. } \\
\text { - New process or facility has few interfaces } \\
\text { with other facilities, or processes. }\end{array}$ \\
\hline 0.5 & $\begin{array}{l}\text { - Components are used in non-standard } \\
\text { ways. } \\
\text { - Requirements are changing. } \\
\text { Design is based on major assumptions } \\
\text { that have a significant impact on the } \\
\text { design output. }\end{array}$ & $\begin{array}{l}\text { Design has many interconnected facets. } \\
\text { - Construction has begun on some parts of } \\
\text { the process or facility without the whole } \\
\text { design being finalized. } \\
\text { - Process or facility must interface with other } \\
\text { processes or facilities to achieve overall } \\
\text { objectives. }\end{array}$ \\
\hline 0.9 & $\begin{array}{l}\text { - } \text { Design is state-of-the-art. } \\
\text { - Research is still on-going. } \\
\text { - } \text { Functional processes have not been built. } \\
\text { - } \\
\text { instead of fact. }\end{array}$ & $\begin{array}{l}\text { - Design is very complex. } \\
\text { - Design and construction are proceeding } \\
\text { almost at the same time. } \\
\text { - Process or facility depends on new and } \\
\text { extensive software. } \\
\text { - Process or facility is a vital part of an } \\
\text { interdependent group of other facilities. }\end{array}$ \\
\hline
\end{tabular}

Next, a magnitude is assigned to the consequence of failure (Cf). Such consequences range from minor inconveniences from which recovery is quick and inexpensive, to technical catastrophes from which recovery, if possible at all, is prolonged and costly. Table 2.2 provides the guidance for assigning the magnitude.

Table 2.2 - Consequences of Failure

\begin{tabular}{|l|l|}
\hline \multicolumn{1}{|c|}{ Magnitude } & \multicolumn{1}{c|}{ Consequences of Failure (Cf) } \\
\hline 0.1 (low) & Minimal, or no consequences, unimportant \\
\hline 0.3 (minor) & Small reduction in technical performance \\
\hline 0.5 (moderate) & Some reduction in technical performance \\
\hline 0.7 (significant) & Degradation in technical performance \\
\hline 0.9 (high) & Technical goal cannot be achieved \\
\hline
\end{tabular}

For all assessments of the technology risk of the waste treatment options, a Cf was chosen equal to 0.7. Should a preferred treatment option suffer a technical failure, it was postulated that the result would be a costly and time-consuming redesign to develop another process to meet requirements. Until the redesign was complete and implemented, waste treatment performance would be significantly degraded.

The maturity and complexity factors are combined with the consequence factor in an equation to give the risk factor $(\mathrm{RF})$ :

$$
\mathrm{RF}=(\mathrm{Pf}+\mathrm{Cf})-(\mathrm{Pf} x \mathrm{Cf})
$$

The resulting risk factor $(\mathrm{RF})$ is a number between 0.19 and 0.99 .

$$
\text { If } \mathrm{Pf}=0.1 \text { and } \mathrm{Cf}=0.1 \text {, then } \mathrm{RF}=(0.1+0.1)-(0.1 \times 0.1)=0.19
$$




$$
\text { If } \mathrm{Pf}=0.9 \text { and } \mathrm{Cf}=0.9 \text {, then } \mathrm{RF}=(0.9+0.9)-(0.9 \times 0.9)=0.99
$$

As can be seen from the above, the closer the RF is to 0.99 the greater the technology risk.

In the model used to screen and evaluate waste treatment options, numbers ranging from 0 to 100 were assigned to treatment option attributes with high numbers representing more desirable features. To make technology risk assessment scores work the same way (high numbers indicating a low technology risk), the risk factor was converted arithmetically to a number between 0 and 100 and called the Technology Success Factor (TSF). A TSF score near 100 indicates a high degree of simplicity and maturity for a treatment option.

In the initial screening of treatment options, those with TSF scores under 50 were discarded. It means only that, at this time, such technologies remain unproved and cannot be recommended in the Site Treatment Plan. Other departments at SRS are investigating and encouraging innovative waste treatment technologies. When these technologies mature, the SRS waste management approach will assess them for the Site's waste treatment program.

\section{In-Depth Options Analysis (IDOA)}

After the elimination of those treatment options with a low possibility for technological success, most waste streams still had several viable treatment options. It became necessary to choose the "best" treatment for each waste stream. To determine the best option, all viable treatment options were subjected to an In-Depth Options Analysis. Comparison among treatment options for a given waste stream is facilitated when each option can be assigned a number that reflects the degree to which the option satisfies a set of criteria or requirements. The method of developing a numerical ranking of treatment options is known as the IDOA model.

The IDOA process took several steps:

1. Attributes by which all treatment processes would be analyzed were determined.

2. The relative importance of the attributes was determined.

3. The IDOA model was applied to each viable treatment option.

4. Engineering assessment took the IDOA model results into account with other factors to determine the Preferred Option to treat a given waste stream.

The categories and attributes analyzed were:

Process Parameters

- Volume alteration

- Secondary waste generation

- Destruction, removal, and demobilization efficiency

- Flexibility

- Ability to be shipped

- Final wasteform

Engineering Parameters

- System implementability

- Availability

- Scalability

- Remedial measures

- Schedule for treatment of waste

Personnel Parameters

- Consequences of unmitigated accident scenarios

- Non operational worker potential exposure

- Operational worker potential exposure 
- Transportation potential exposure

Regulatory Parameters

- Need for a variance

- Ability to obtain a permit

- Waste disposal

Public Acceptance

- Public acceptance

Cost Considerations

- Life-cycle cost

- Funding availability

Industry Involvement

- Market for technology

- Private sector involvement

"Enabling statements," clarifying the above attributes, assisted with the process expert's evaluation of treatment options. The "enabling statements" appear in Table 2.3. The attributes and enabling statements formed the basis with which "viable" treatment processes were assessed and compared.

To evaluate a viable treatment option, a team of waste treatment process experts applied the enabling statements to each option. The team assigned a number from 0 (low) to 100 (high) to each attribute. The score reflected the experts' assessment of how well the process satisfied the requirement posed by the attribute.

For example, consider the attribute of "Secondary Waste Generation". If the process produced a small quantity, all of which could be handled by existing technologies, the process experts would give the process a "high" numerical rating (median 80). If the process produced as much as $10 \%$ additional waste that existing technologies could handle, the process experts rated it "medium" (median 50). If the process produced large amounts of secondary waste, or if existing technologies could not handle the secondary waste, the experts rated it "low" (median 20). If the experts felt a score other than the median better reflected conditions, they could assign another number, provided they gave an explanation for the variation (e.g., in the preceding case, if the process produced $20 \%$ additional secondary waste, the evaluation would include a statement such as "subtract 10 points because of additional waste generation").

For the cost attribute, a team of cost estimators determined the life-cycle cost. The estimators developed:

- Operating and maintenance cost for the life of the facility

- Disposal cost of all final wasteforms in compliance with LDR

- Decontamination and decommissioning cost to return the facility to a safe and environmentally benign condition at the end of its useful life.

The process experts' evaluation resulted in a raw technical score for each attribute, and inclusion of the cost estimators' life-cycle cost data resulted in a raw total score. Nevertheless, these raw scores did not reflect the relative importance of the attributes. The Technical Advisory Committee (TAC), a group of experienced technical experts with backgrounds in engineering design, environmental protection, process technology, safety, and health, was appointed to oversee the treatment selection process. They recognized that not applying a weighting factor to each attribute assigned the same weight to all of them. So, the Technical Advisory Committee proposed a weight for each factor. The weighting factors were then reviewed and modified by independent reviewers, regulators, and a citizens' focus group. The final weight factors appear in Table 2.3.

Each option's weighted technical scores were summed. The total fell between 0 (least preferable) and 100 (most preferable). The sums enabled the treatment option to be ranked according to the technical weighted 
score. Then, the weighted life-cycle cost data were added to the technical weighted score in a way that ensured that the cost of a treatment facility was equitably apportioned among the waste streams that would be processed using that facility. This resulted in a total weighted score. The IDOA model generated the technical and total weighted scores for each treatment option. These IDOA model scores were useful tools to narrow the entire population of options.

- The IDOA model ensured the same attributes were analyzed for every process or facility.

- The IDOA model provided some guidance to help make analyses consistent among the facilities.

- The IDOA model enhanced the engineering assessment by incorporating consistent structure and logic.

Application of the IDOA model ensures consistency and completeness in performing the in-depth analysis of the potential treatment options associated with each waste stream. The primary function of the model is to lower the number of possible treatment options to a more manageable number for further analysis and review. The model was not developed to provide a clear PO winner, and the reader is cautioned against believing that the PO having the best model score is the PO of choice. On the contrary, the application of the model results in a smaller set of POs that may have model scores within a 10 to $15 \%$ range of each other that serve as the focus of further analysis. It was not expected, and in practice has not always been the case, that the treatment with the best model score is the PO of choice.

Sixteen of the waste streams also have treatment options proposed by outside vendors. Many of these options, however, remain technologically unproven. The vendors have offered to perform studies to demonstrate that their technology can produce a wasteform that will meet LDRs. A separate task team is working with the vendor proposals to determine which technologies appear worthy of further investigation. As rapidly as procurement rules allow, and as completely as budgetary constraints permit, contracts are being made with vendors to pursue the most promising innovative treatment methods.

Nonetheless, the technical viability of these technologies has been assumed, and hypothetical vendor processes have been projected, to permit application of the IDOA model and a comparison of the potential vendor processes with other treatment options. In the months ahead, successful vendors' studies will be translated into process designs that can be compared with the preferred options selected. This comparison will verify the conclusions drawn from the potential vendors' processes and may reveal a vendor treatment technology for a waste stream that is preferable to the option previously favored. 
Table 2.3 - Attributes and Enabling Statements for Options Analysis

\begin{tabular}{|c|c|c|c|c|}
\hline Wt. & Attribute & $\begin{array}{c}\text { High } \\
\text { Score } \\
\text { Median } 80\end{array}$ & $\begin{array}{c}\text { Medium } \\
\text { Score } \\
\text { Median } 50\end{array}$ & $\begin{array}{c}\text { Low } \\
\text { Score } \\
\text { Median } 20\end{array}$ \\
\hline $22 \%$ & \multicolumn{4}{|c|}{ PROCESS PARAMETERS } \\
\hline $5 \%$ & Volume Alteration & $\begin{array}{l}\text { A factor of } 5 \text { reduction of waste } \\
\text { occurs. }\end{array}$ & $\begin{array}{l}\text { The volume is maintained at } 1: 1 \\
\text { after processing. }\end{array}$ & $\begin{array}{l}\text { The volume is increased by a factor of } 2 \\
\text { or more after processing. }\end{array}$ \\
\hline $4 \%$ & $\begin{array}{l}\text { Secondary Waste } \\
\text { Generation }\end{array}$ & $\begin{array}{l}\text { A small quantity is produced, all } \\
\text { of which can be handled by } \\
\text { existing technologies. }\end{array}$ & $\begin{array}{l}\text { An additional amount of waste, in } \\
\text { the range of } 10 \% \text {, is generated, } \\
\text { which can be handled by existing } \\
\text { technologies. }\end{array}$ & $\begin{array}{l}\text { Large quantities are produced, or } \\
\text { existing technologies are not available } \\
\text { for treatment. }\end{array}$ \\
\hline $2 \%$ & $\begin{array}{l}\text { Destruction } \\
\text { Removal, and } \\
\text { Demobilization } \\
\text { Efficiency }\end{array}$ & $\begin{array}{l}\text { All applicable LDR standards are } \\
\text { met. }\end{array}$ & $\begin{array}{l}\text { Additional LDR treatment is } \\
\text { required for some of the } \\
\text { constituents; technology exists. }\end{array}$ & $\begin{array}{l}\text { Additional treatment is required to meet } \\
\text { requirements, and technology does not } \\
\text { exist, or requires modification. }\end{array}$ \\
\hline $3 \%$ & Flexibility & $\begin{array}{l}\text { The process can treat waste } \\
\text { streams of similar compositions } \\
\text { to that assumed as a design basis } \\
\text { without producing a final } \\
\text { wasteform that fails to meet } \\
\text { requirement. The process does } \\
\text { not need to be reconfigured or } \\
\text { monitored with special care to } \\
\text { meet throughput specifications. }\end{array}$ & $\begin{array}{l}\text { The process can treat waste } \\
\text { streams of similar compositions } \\
\text { to that assumed as a design basis } \\
\text { without producing a final } \\
\text { wasteform that fails to meet } \\
\text { requirement; but the process must } \\
\text { either be reconfigured or } \\
\text { monitored with special care to } \\
\text { meet throughput specifications. }\end{array}$ & $\begin{array}{l}\text { The process cannot treat waste streams } \\
\text { of compositions that differ from that } \\
\text { assumed as a design basis. Special care } \\
\text { must be taken to monitor influent } \\
\text { streams to ensure that they conform to } \\
\text { the composition assumed as a design } \\
\text { basis. }\end{array}$ \\
\hline $2 \%$ & $\begin{array}{l}\text { Ability to be } \\
\text { Shipped }\end{array}$ & $\begin{array}{l}\text { Treatment residuals meet } \\
\text { shipping requirements without } \\
\text { any additional treatment. }\end{array}$ & $\begin{array}{l}\text { Treatment residuals require } \\
\text { simple physical treatment to meet } \\
\text { shipping requirements. }\end{array}$ & $\begin{array}{l}\text { Treatment residuals require extensive } \\
\text { treatment to meet shipping requirements } \\
\text { or technologies do not exist. }\end{array}$ \\
\hline $6 \%$ & Final Wasteform & $\begin{array}{l}\text { Wasteform meets the expected } \\
\text { disposal WAC. }\end{array}$ & $\begin{array}{l}\text { Final forms require additional } \\
\text { treatment to meet disposal WAC; } \\
\text { technologies exist. }\end{array}$ & $\begin{array}{l}\text { A significant additional treatment is } \\
\text { required before disposal or technologies } \\
\text { do not exist. }\end{array}$ \\
\hline $20 \%$ & \multicolumn{4}{|c|}{ ENGINEERING PARAMETERS } \\
\hline $13 \%$ & $\begin{array}{l}\text { System Implement- } \\
\text { ability }\end{array}$ & $\begin{array}{l}\text { Most of the elements and } \\
\text { processes have been previously } \\
\text { demonstrated on similar uses and } \\
\text { applications. }\end{array}$ & $\begin{array}{l}50 \% \text { or fewer of the elements } \\
\text { have been previously } \\
\text { demonstrated on similar uses and } \\
\text { applications. }\end{array}$ & $\begin{array}{l}\text { Few or none of the elements have been } \\
\text { demonstrated. }\end{array}$ \\
\hline $3 \%$ & Availability & $\begin{array}{l}\text { Key components arranged in } \\
\text { similar systems have resulted in } \\
\text { availability greater than } 80 \% .\end{array}$ & $\begin{array}{l}\text { Process is expected to be } \\
\text { available about } 50 \% \text { of the time. }\end{array}$ & $\begin{array}{l}\text { Process is expected to be available about } \\
20 \% \text { of the time, or large uncertainties } \\
\text { exist in ability to predict availability. }\end{array}$ \\
\hline $1 \%$ & Scalability & $\begin{array}{l}\text { Process can be easily expanded to } \\
\text { take advantage of economies of } \\
\text { scale. Also, processes go from } \\
\text { laboratory scale directly to plant } \\
\text { scale. }\end{array}$ & $\begin{array}{l}\text { Process can accept a range of } \\
\text { input but has limitations for } \\
\text { expansion. Also, pilot scale tests } \\
\text { are required before plant-scale } \\
\text { design. }\end{array}$ & $\begin{array}{l}\text { Process cannot be expanded to take } \\
\text { advantage of economies of scale. Also, } \\
\text { laboratory or pilot scale testing would be } \\
\text { impractical, or not yield meaningful } \\
\text { results. Plant-scale design must come } \\
\text { directly from engineering calculations. }\end{array}$ \\
\hline $1 \%$ & Remedial Measures & $\begin{array}{l}\text { Process failure or malfunction } \\
\text { does not create a waste that } \\
\text { cannot be treated by other means; } \\
\text { alternative treatment methods for } \\
\text { the original waste exist and can } \\
\text { be implemented within three } \\
\text { months of recognition of need. }\end{array}$ & $\begin{array}{l}\text { Process failure or malfunction } \\
\text { creates other wastes that must be } \\
\text { characterized to determine } \\
\text { treatability; alternative treatment } \\
\text { methods must be developed to } \\
\text { treat new waste created by the } \\
\text { process malfunction. }\end{array}$ & $\begin{array}{l}\text { Process failure or malfunction creates } \\
\text { other wastes for which there is no } \\
\text { known treatment; no alternative methods } \\
\text { for treatment of original waste exist. }\end{array}$ \\
\hline $1 \%$ & $\begin{array}{l}\text { Schedule for } \\
\text { Treatment of Waste }\end{array}$ & $\begin{array}{l}\text { A schedule for addressing and } \\
\text { processing waste can be } \\
\text { determined with high confidence. }\end{array}$ & $\begin{array}{l}\text { Some technology issues can } \\
\text { produce uncertainty in schedule } \\
\text { development. System } \\
\text { complexities may prolong } \\
\text { schedule. }\end{array}$ & $\begin{array}{l}\text { Availability, technology or flexibility } \\
\text { issues severely limit confidence in } \\
\text { developing schedules. Extensive } \\
\text { training, system, and operational } \\
\text { complexity may also create problems. }\end{array}$ \\
\hline
\end{tabular}


Table 2.3 - Attributes and Enabling Statements for Options Analysis (cont'd)

\begin{tabular}{|c|c|c|c|c|}
\hline Wt. & Attribute & $\begin{array}{c}\text { High } \\
\text { Score } \\
\text { Median } 80\end{array}$ & $\begin{array}{c}\text { Medium } \\
\text { Score } \\
\text { Median } 50\end{array}$ & $\begin{array}{c}\text { Low } \\
\text { Score } \\
\text { Median } 20\end{array}$ \\
\hline $20 \%$ & \multicolumn{4}{|c|}{ PERSONNEL PARAMETERS } \\
\hline $6 \%$ & $\begin{array}{l}\text { Consequence of } \\
\text { Unmitigated } \\
\text { Accident Scenarios }\end{array}$ & $\begin{array}{l}\text { There are little or no facility } \\
\text { emissions for routine operations } \\
\text { under all but the most } \\
\text { catastrophic accidents. }\end{array}$ & $\begin{array}{l}\text { There are little or no emissions for } \\
\text { routine operations, but significant } \\
\text { releases occur under most accident } \\
\text { scenarios. }\end{array}$ & $\begin{array}{l}\text { There are marginally acceptable releases } \\
\text { under routine operations or extensive } \\
\text { releases under most accident scenarios. }\end{array}$ \\
\hline $6 \%$ & $\begin{array}{l}\text { Non-Operational } \\
\text { Worker Potential } \\
\text { Exposure }\end{array}$ & $\begin{array}{l}\text { Significantly fewer workers } \\
\text { required to construct and } \\
\text { decommission a facility with the } \\
\text { proposed process as compared } \\
\text { to other technologies. There is } \\
\text { lower than average non-routine } \\
\text { maintenance. }\end{array}$ & $\begin{array}{l}\text { Average number of workers and } \\
\text { non-routine maintenance required. }\end{array}$ & $\begin{array}{l}\text { The process is more complex than } \\
\text { average facility construction. Non- } \\
\text { routine maintenance and } \\
\text { decommissioning is required. }\end{array}$ \\
\hline $6 \%$ & $\begin{array}{l}\text { Operational Worker } \\
\text { Potential Exposure }\end{array}$ & $\begin{array}{l}\text { There are significantly fewer } \\
\text { workers potentially exposed or } \\
\text { the potential exposure is much } \\
\text { lower than average. }\end{array}$ & $\begin{array}{l}\text { There are an average number of } \\
\text { workers and potential exposure } \\
\text { levels. }\end{array}$ & $\begin{array}{l}\text { There are a greater than average number } \\
\text { of workers or there is a greater than } \\
\text { average potential exposure to the work } \\
\text { force. }\end{array}$ \\
\hline $2 \%$ & $\begin{array}{l}\text { Transportation } \\
\text { Potential Exposure }\end{array}$ & $\begin{array}{l}\text { No transportation of treated or } \\
\text { untreated waste is required. }\end{array}$ & $\begin{array}{l}\text { Limited additional characterization } \\
\text { is required to support } \\
\text { transportation, no new packaging/ } \\
\text { certification facilities required, and } \\
\text { limited number of waste transports } \\
\text { are required. }\end{array}$ & $\begin{array}{l}\text { Significant additional waste } \\
\text { characterization is required for } \\
\text { transportation, new packaging/ } \\
\text { certification facilities are required, a } \\
\text { large number of waste transports are } \\
\text { needed, or a large number of miles are } \\
\text { required for each waste shipment. }\end{array}$ \\
\hline $14 \%$ & \multicolumn{4}{|c|}{ REGULATORY PARAMETERS } \\
\hline $4 \%$ & Need for Variance & $\begin{array}{l}\text { Processes are in full compliance } \\
\text { with all applicable regulations } \\
\text { with little or no difficulty or } \\
\text { with no process modifications. }\end{array}$ & $\begin{array}{l}\text { Processes are in partial compliance } \\
\text { with all applicable regulations with } \\
\text { little or no difficulty. Full } \\
\text { compliance may be achieved } \\
\text { through requests for variances or } \\
\text { with limited modifications to the } \\
\text { process. }\end{array}$ & $\begin{array}{l}\text { Majority of the applicable regulations } \\
\text { cannot be met without vast } \\
\text { modifications to the process or other } \\
\text { extensive variances. }\end{array}$ \\
\hline $6 \%$ & $\begin{array}{l}\text { Ability to Obtain a } \\
\text { Permit }\end{array}$ & $\begin{array}{l}\text { Permitting process is well- } \\
\text { defined and relevant precedents } \\
\text { for success have been } \\
\text { established. Similar processes } \\
\text { have been previously permitted } \\
\text { by the regulatory agencies } \\
\text { (primarily SCDHEC) with little } \\
\text { or no difficulty. }\end{array}$ & $\begin{array}{l}\text { Process or key elements have been } \\
\text { permitted elsewhere, but some key } \\
\text { differences may exist (for example, } \\
\text { differences in waste streams, or } \\
\text { waste stream characterization). } \\
\text { Similar processes have been } \\
\text { previously permitted by the } \\
\text { regulatory agencies (primarily } \\
\text { SCDHEC) with moderate } \\
\text { difficulty. }\end{array}$ & $\begin{array}{l}\text { The process is unproved technology or a } \\
\text { new arena of application or the need for } \\
\text { multiple permits builds in substantial } \\
\text { permitting barriers. Similar processes } \\
\text { have been previously permitted by the } \\
\text { regulatory agencies (primarily } \\
\text { SCDHEC) with extreme difficulty or } \\
\text { have never been previously permitted. }\end{array}$ \\
\hline $4 \%$ & Waste Disposal & $\begin{array}{l}80 \% \text { of both primary and } \\
\text { secondary wastes have been } \\
\text { rendered non-hazardous. The } \\
\text { other } 20 \% \text { remain hazardous. }\end{array}$ & $\begin{array}{l}50 \% \text { of both primary and } \\
\text { secondary wastes have been } \\
\text { rendered non-hazardous. The other } \\
50 \% \text { remain hazardous. }\end{array}$ & $\begin{array}{l}80 \% \text { of both primary and secondary } \\
\text { wastes remain hazardous. The other } 20 \% \\
\text { have been rendered non-hazardous. }\end{array}$ \\
\hline
\end{tabular}


Table 2.3 - Attributes and Enabling Statements for Options Analysis (cont’d)

\begin{tabular}{|c|c|c|c|c|}
\hline $\mathbf{W t}$. & Attribute & $\begin{array}{c}\text { High } \\
\text { Score } \\
\text { Median } 80\end{array}$ & $\begin{array}{l}\text { Medium } \\
\text { Score } \\
\text { Median } 50\end{array}$ & $\begin{array}{c}\text { Low } \\
\text { Score } \\
\text { Median } 20\end{array}$ \\
\hline $9 \%$ & \multicolumn{4}{|c|}{ PUBLIC ACCEPTANCE } \\
\hline $9 \%$ & Public Acceptance & $\begin{array}{l}\text { Stakeholders accept the process } \\
\text { and the risks. Similar processes } \\
\text { have been publicly } \\
\text { acknowledged by stakeholders as } \\
\text { being acceptable. }\end{array}$ & $\begin{array}{l}\text { Some stakeholder concerns that } \\
\text { could affect successful utilization } \\
\text { of the technology. Stakeholders } \\
\text { have publicly stated reservations } \\
\text { about the safety or effectiveness of } \\
\text { similar processes. }\end{array}$ & $\begin{array}{l}\text { Significant stakeholder concerns about } \\
\text { process. Stakeholders have publicly stated } \\
\text { disapproval about the safety or } \\
\text { effectiveness of similar processes, or } \\
\text { stakeholder opinion is unknown. }\end{array}$ \\
\hline $15 \%$ & \multicolumn{4}{|c|}{ COST CONSIDERATIONS } \\
\hline $14 \%$ & \multicolumn{4}{|c|}{$\begin{array}{l}\text { Life-cycle Cost } \\
\text { Costs Developed According To DSTP Cost Guidance Rev. } 1 \text {. } \\
\text { Costs are estimated for } \\
\text { pre-operating costs } \\
\text { facility costs } \\
\text { operating and maintenance costs } \\
\text { disposal cost } \\
\text { decontamination and decommissioning costs } \\
\text { The SUM of the above costs is assigned a score in proportion to where it falls between } \$ 1 \text { and } \$ 35 \text { million. The higher the cost, the lower } \\
\text { the score. Any cost totaling more than } \$ 35 \text { million receives a score of zero. }\end{array}$} \\
\hline $1 \%$ & Funding Availability & $\begin{array}{l}\text { Life-cycle costs can be supported } \\
\text { within target budget. }\end{array}$ & $\begin{array}{l}\text { Life-cycle costs can be supported } \\
\text { with less than } 10 \% \text { increase in } \\
\text { target funding levels. }\end{array}$ & Line item funding required at high-levels. \\
\hline $1 \%$ & \multicolumn{4}{|c|}{ INDUSTRY INVOLVEMENT } \\
\hline $0.5 \%$ & $\begin{array}{l}\text { Market for } \\
\text { Technology }\end{array}$ & $\begin{array}{l}\text { Numerous markets are identified } \\
\text { within and outside DOE. More } \\
\text { than three DOE and commercial } \\
\text { nuclear facilities have similar } \\
\text { wastes. }\end{array}$ & $\begin{array}{l}\text { More than one market is } \\
\text { identified within and outside } \\
\text { DOE. Two DOE and commercial } \\
\text { nuclear facilities have similar } \\
\text { wastes. }\end{array}$ & $\begin{array}{l}\text { No markets or needs are identified. SRS } \\
\text { waste is unique. }\end{array}$ \\
\hline $0.5 \%$ & $\begin{array}{l}\text { Private Sector } \\
\text { Involvement }\end{array}$ & $\begin{array}{l}\text { A private sector technology } \\
\text { company is identified with } \\
\text { experience and interest and the } \\
\text { company has experience in } \\
\text { permitting activities. A vendor has } \\
\text { submitted a proposal and has } \\
\text { permitting experience. }\end{array}$ & $\begin{array}{l}\text { A private sector party has } \\
\text { expressed an interest; however, } \\
\text { has little or no experience in this } \\
\text { type of activity or permitting } \\
\text { process. A vendor with non- } \\
\text { technical experience has } \\
\text { submitted a proposal. }\end{array}$ & $\begin{array}{l}\text { No private sector companies have } \\
\text { expressed an interest or a need for the } \\
\text { technology. }\end{array}$ \\
\hline
\end{tabular}

\section{Engineering Assessment}

The last step in the IDOA was to perform an engineering assessment, taking into account the score generated by the IDOA model. While application of the IDOA model analyzed the degree to which the treatment option satisfied the requirements of the prescribed attributes, engineering assessment took a broader perspective, considering factors that combine to identify the preferred treatment option.

\subsection{Coordination with Regulatory Agencies and Other Stakeholders}

\section{Coordination with Regulatory Agencies}

The Federal Facility Compliance Act (FFCAct) offered an opportunity for DOE and the state and EPA regulators who approved the plans to work cooperatively toward defining mixed waste treatment strategies. As requested by the states, DOE signed a cooperative agreement in August 1993 with the National Governors Association (NGA) to facilitate the DOE-to-state interactions. The NGA has sponsored national meetings on a routine basis with DOE, the states, EPA, and the Indian Nations throughout development of the STPs. 


\section{Public Participation}

The FFCAct requires the states and EPA to provide for public involvement after the Proposed Plans are submitted. DOE has provided additional opportunities for public input into the development of Conceptual Site Treatment Plans (CSTP) and Draft Site Treatment Plans (DSTP) through existing public involvement mechanisms at the site.

The public has been informed and invited to participate throughout the STP development process. In December 1993, a CSTP fact sheet was mailed to stakeholders on the Site's public involvement distribution list. In response to the fact sheet, citizens volunteered to participate in a focus group to look at three STP development documents: the Site Treatment Plan Assumption List, Site Treatment Plan Development Flowchart, and Site Treatment Plan In-Depth Options Analysis Model.

The focus group, which consisted of volunteers from the general public and members of the Citizens Advisory Board (CAB), met on May 9, 1994, to give comments on the documents. Representatives of SCDHEC also attended the meeting. SRS considered the comments and made revisions to the DSTP based on the expressed concerns.

The DSTP also was discussed at the SRS Waste Management Environmental Impact Statement (WMEIS) informational workshops held in April 1994 and the WMEIS scoping hearings held in May 1994.

When the DSTP was issued, SRS also issued a fact sheet summarizing the highlights of the plan and conducted DSTP public workshops and briefings for special interest groups. Information about other sites that identified SRS as a preferred option for the treatment of their mixed waste streams was provided. A public workshop was held in Aiken on the afternoon and evening of October 4, 1994. In addition, an edited videotape of the workshop was carried on cable channels in Augusta, Columbia, and Savannah. Showings of the video were given on October 11, 12, and 13. After each presentation SRS personnel were available to answer questions and take comments over a toll-free number that was flashed on the screen at the time of the video viewing.

Copies of the Savannah River Site DSTP and executive summary and other sites' DSTPs were placed in the Public Reading Room at the University of South Carolina (USC) Aiken library. The plan's availability and public workshops were announced through public service announcements, newspaper, television and radio advertisements, and news releases using the Site's media list. Copies of the DSTP were mailed to stakeholders upon request.

SRS representatives offered briefings on the highlights of the DSTP to interested community groups. Stakeholders attending the public workshops were invited to give comments at the workshop or to provide them later. Stakeholders who attended the public workshop or called on the toll-free number after the videotape viewings were invited to participate in focus group meetings to provide further comment on the DSTP. Focus group meetings were held on October 18, 20, and 26. Although sparsely attended, some valuable input was provided and incorporated into the PSTP. Comments, also accepted through the mail, were considered in the development of the Proposed STP (PSTP).

Copies of the PSTP, Executive Summary, and other sites' plans were placed in the Public Reading Room at USC-Aiken. The public was made aware of the plan's availability through public service announcements, newspaper, television and radio advertisements, and news releases using the site's media list. A revised fact sheet was developed and issued to stakeholders. Stakeholders were informed that comments on the PSTP could be submitted to SCDHEC.

The PSTP was submitted to SCDHEC on March 30, 1995. Under requirements of the FFCAct, SCDHEC then assumed responsibility for public notice. SCDHEC performed an internal review and put a modified PSTP out for a 45-day public review and comment period beginning on July 14, 1995. The public notice period concluded with a public hearing held on August 30, 1995. SCDHEC reviewed public comments and requested changes to the PSTP where appropriate. 
SCDHEC requested changes to the PSTP as a result of responses from the public as well as its own review. During September 1995, SRS and SCDHEC combined discussion on language for the Consent Order and changes to the PSTP. On September 20, 1995, SCDHEC approved the PSTP with modification and issued a proposed Consent Order 95-22-HW for the implementation of the STP. SRS submitted the requested modifications. The Consent Order was signed by all parties and became effective on September 29, 1995, after which time the modified PSTP became the approved STP or, simply, the STP.

The Consent Order 95-22-HW includes a provision for public notice and comment on changes that SRS may propose to waste stream treatments in future modifications to the STP. This helps to keep stakeholders aware of future change in treatment strategies as technologies evolve.

\section{Conclusion}

The Savannah River Site developed an aggressive and active public participation plan, which comprehensively included surrounding communities, regulatory agencies, and other identified stakeholders. The overall purpose was to ensure the public participation program for the STP was proactive, responsive to public concerns, and serves the best interests of stakeholders and the DOE. Activities were designed to meet the overall program objectives, coordinate with other activities, and provide opportunity for meaningful public involvement.

\section{National Level}

At the national level, DOE presented information on the development of the STPs to the Environmental Management Advisory Board, and held an open house in Washington, DC when the Draft Plans were released. DOE also met informally with representatives of Indian tribes and separately with representatives of other groups that had interest in Site Treatment Plan development. The purpose of the meeting was to determine if there were national issues that had not been identified through site-specific activities. Additional opportunities to obtain input at the national level may be offered in coordination with the states and EPA. The Center for Environmental Management provides information on Act activities at the national level (1-800-736-3282; 202863-5084 in Washington, DC).

\subsection{Mixed Waste Characterization}

\section{General}

Westinghouse Savannah River Company (WSRC) is responsible for day-to-day management and operation of the waste management programs for the Department of Energy. DOE provides oversight and overall direction for solid waste management programs at SRS.

The process for defining and determining whether a waste material or stream is hazardous or nonhazardous is defined in the WSRC Environmental Compliance Manual (ECM) Procedure 6.03 as well as the Waste Acceptance Criteria Manual, 1S. The requirements of the ECM are applicable to WSRC and its subcontractors handling wastes and making the determination of whether the wastes are hazardous or nonhazardous as defined by the federal Resource Conservation and Recovery Act and the South Carolina Hazardous Waste Management Regulations. Specific guidance and requirements for making these determinations are provided in the SRS Waste Disposal Manual, WSRC-IM-90-138. By Memoranda of Understanding, other site organizations such as the U. S. Forest Service have agreed to abide by WSRC requirements when WSRC services or facilities are utilized.

As described below, SRS is composed of several major facilities, each with its own operating and support organizations. A number of these organizations play a role in characterizing waste at SRS. 


\section{Facility Management and Environmental Compliance Authorities}

Facility Management ensures the facility is in compliance with all applicable federal/state regulations and site requirements. This includes management of waste generated and stored at the facility, including characterization of the waste prior to shipment to an onsite or offsite waste storage, treatment, or disposal facility.

Each major facility, group of facilities, or operating organization has a designated Environmental Compliance Authority (ECA) to advise and assist facility management in developing and maintaining the facility's environmental programs. The ECAs are individuals knowledgeable of environmental regulations and how the regulations apply to those facilities for which the ECAs are responsible.

ECM Procedure 6.03 requires the ECA or department representative at the facility or area generating a waste first to determine whether a waste is hazardous. If information to determine that a waste is hazardous is unavailable or inadequate, the waste is sampled and analyzed, provided sampling and analysis does not result in excess exposure of personnel to radiation.

The facility or area generating a waste also is responsible for preparing a waste characterization form for each routinely generated waste stream. The completed form is submitted to the Solid Waste Division (SWD) Department. The generator of a new waste must work closely with SWM and the Environmental Services Section (ESS) to ensure the new waste can be managed under existing permits and that adequate onsite or offsite storage, treatment, and disposal capacity is available; or that, until sufficient waste volume is generated, satellite accumulation areas and/or 90-day staging areas are established in compliance with RCRA regulations. The generator also is responsible for determining appropriate EPA/SCDHEC hazardous waste codes and assigning appropriate SRS Hazardous Waste Index (HWI) number(s) for quarterly hazardous waste reporting purposes. A waste characterization form also must be completed when a new hazardous waste stream is generated or a hazardous waste generation process has changed.

\section{Environmental Services Section (ESS) and Office of General Counsel (OGC)}

The ESS is the WSRC organization responsible for coordinating and overseeing sitewide environmental protection programs and assisting operating organizations with compliance issues including waste characterization. The WSRC OGC is consulted in all matters pertaining to environmental compliance that may have legal implications.

The SRS Waste Disposal Manual, prepared by ESS, includes a section on the identification and characterization of hazardous waste. The manual summarizes the applicable federal and state environmental regulations and provides site guidance for identifying, characterizing, managing, transporting, treating, storing, and disposing of mixed, hazardous, and nonhazardous waste. In addition, the Waste Disposal Manual provides guidance for waste minimization and environmental training.

The ESS issues regulatory guidance in the form of letters and memoranda to various site organizations to address specific regulatory questions as they arise. Many of these memoranda and letters are issued to provide guidance on the proper classification of a waste. These memoranda and letters are included in an appendix to the Waste Disposal Manual. The manual is updated periodically to incorporate changes in the regulations and add newly issued internal guidance documents. These periodic updates are issued to the custodians of each copy of the Waste Disposal Manual through the WSRC Document Control Section.

\section{Environmental Monitoring and Analysis}

The Environmental Monitoring and Analysis Group (EMA) serves as the primary resource to various site waste generators during the preliminary waste identification and characterization phase. EMA provides hazardous waste sampling services conducted in accordance with a sampling plan. These sampling plans are developed to ensure that sampling is representative, that sample collection and shipping meet regulatory protocols, and that proper analytical methods are requested. Alternatively, site organizations may collect their own samples. EMA 
offers consultation services to those organizations. Technical support is available to waste generators for sampling activities involving radioactive wastes. EMA has also developed sitewide sampling guidance. EMA directs samples to onsite laboratories for radiological screening prior to offsite shipments and they also facilitate the shipment of samples to offsite laboratories for analysis. To the extent possible, EMS sends hazardous waste samples it collects to SCDHEC-certified laboratories. However, in some cases, because of high radioactivity levels or need for specialized analytical techniques, analyses are conducted onsite. Assistance on the statistical aspects of a sampling plan can be obtained from the Applied Statistics Group, Scientific Computations Section of the Savannah River National Laboratory.

\section{Solid Waste and Infrastructure Division}

The Solid Waste and Infrastructure Division (SWI) is responsible for management of many of the facilities for waste treatment, storage, and disposal at SRS. SWI also coordinates all offsite shipment and disposal of hazardous waste. The Site Treatment Plan is managed by SWI.

SWI issued the SRS Waste Acceptance Criteria Manual (1S Manual) for developing a waste classification system for managing each waste type, establishing waste acceptance criteria (WAC) for storage and disposal facilities, and instituting a Waste Certification Program to assure the waste received for treatment, storage, or disposal at SWI facilities meets the waste acceptance criteria (WAC).

The 1S Manual requires each generator that delivers waste to treatment, storage, or disposal facilities to implement a Waste Certification Program. This program provides assurance that the requirements for waste acceptance by the receiving facility are met. Waste certification provides assurance that waste has been properly identified, characterized, segregated, packaged, and shipped to the appropriate receiving facility in accordance with that receiving facility's waste acceptance criteria (WAC). Under this program, each waste generator designates a Generator Certification Official (GCO) to administer the waste generator's certification program and to assure that the waste generator's waste management programs implement and document controls to meet established waste acceptance criteria.

The SWI reviews and assesses a waste generator's certification plan, characterization methodology, other documentation, and procedures to assure compliance with the certification plan and TSD facility requirements. The SWI Department is responsible for performing surveillances, audits, or assessments of the waste generator's waste certification program as needed and for providing guidance and assistance.

\section{Process Knowledge, Sampling, and Analysis}

Hazardous waste management regulations obligate the generator of a solid waste to "determine if that waste is a hazardous waste." The generator may accomplish this by testing the waste according to the methods set forth in Subpart C, or according to an equivalent method approved under 40 CFR 260.21. The regulations also allow the generator to apply "knowledge of the hazard characteristic of the waste in light of the materials or the processes used" to make the hazardous waste determination. This approach is generally referred to as a "process knowledge" determination.

Guidance has been provided to SRS waste generators in both the Waste Disposal and 1S Manuals that the ideal way to determine if a waste is characteristically hazardous is by collecting and analyzing a representative sample of the waste. Generators are directed to Test Methods for Evaluating Solid Waste, Physical/Chemical Methods (EPA Publication SW-846, Third Edition, November 1986) for the methods necessary to ensure that a sampling program meets this objective. SW-846 cautions against the "haphazardly selected sample". As indicated above, technical support to waste generators is available from the EMA for sampling activities involving radioactive wastes. EMA also provides technical review services for waste characterization analytical data.

Although generators are strongly encouraged to make hazardous waste determinations based on representative samples, it is recognized that this is not always possible. Many of the waste streams onsite are nonhomogeneous job control or debris type waste making it extremely difficult to obtain a sample that is conclusively "representative." 
To supplement information provided in SW-846, SRS has developed internal procedures to provide instructions to waste sampling personnel for collecting representative samples. This sampling procedure has been developed by the Analytical Laboratories Section and is found in the Westinghouse Savannah River Company procedure manual L3.13, PRR $4326 \mathrm{~J}$.

Some SRS waste streams contain levels of radioactivity sufficient to make sampling prohibitively expensive or prevent strict adherence with the sampling and analytical protocols in SW-846. For waste streams such as these, the provision to allow characterization by process knowledge is exceptionally important when the unique difficulties presented by the radioactive component of the waste are considered. Paramount among these difficulties is the control of radiation exposure of personnel during collection, packaging, transportation, and analysis of samples.

An overriding principle of working with radioactive materials is maintaining personnel exposure to radiation at levels that are "as low as reasonably achievable" or ALARA. This principle includes not only exposure of the whole body or extremities to external sources of radiation but also control of surface and airborne radioactive contamination to prevent exposures through inhalation, skin absorption, or ingestion of the radioactive materials. The presence of radioactivity also adds other administrative and regulatory requirements to transporters who must comply with Department of Transportation regulations for the transport of radioactive materials. Commercial laboratories that analyze mixed waste samples must be properly licensed to receive, analyze, and dispose of radioactive materials. The processing and disposal of hazardous waste that is also radioactive requires additional specialized equipment, handling, and technologies which adequately address the radioactivity concerns in addition to the regulatory requirements for hazardous constituents.

Approximately $95 \%$ of the total volume of mixed waste being generated or currently in storage at SRS is characterized by sampling and analysis. In addition, a number of streams are hazardous for toxic metals that are used for their unique properties, and their classification is relatively straightforward. Thus, there is a high degree of confidence that approximately $75 \%$ of the current or past wastes are appropriately classified. However, it is possible that some of the listed waste streams (for example, solvent rags used for cleaning and decontamination) that have not been sampled may contain trace quantities of toxic metals. Where this is known to be a possibility, other waste codes that are thought to be appropriate have been conservatively added to those waste streams.

\section{Radiological Characterization}

A variety of methods are used to characterize the radioactive component of mixed waste. This includes hand held portable monitoring instruments used by Health Protection personnel to conduct measurements of radioactivity levels in the work environment. These instruments are capable of measuring alpha, beta, neutron, and gamma radiation. Although less sophisticated and less precise than laboratory measurements of waste samples, this instrumentation provides the means to quantify the level of radioactivity in mixed waste for the purpose of controlling exposure of personnel to levels that are ALARA. Field measurements can also be used to provide a conservative estimate of the amount of radioactivity present. More precise determination of the amount and type of radioactive material present in a waste material can be made by analyzing a representative sample of the material in a counting or radiochemical laboratory. The sample may or may not be prepared using various chemical separation, purification, and concentration techniques to enhance the overall sensitivity of the analytical technique. Typical laboratory instruments used to analyze or count prepared samples include gas-flow proportional counters for analysis of alpha and nonvolatile beta emitters, liquid scintillation counters for use in analyzing for low energy beta emitters such as tritium, silicon surface barrier detectors used for alpha particle spectroscopy measures, and high-purity germanium detectors used for gamma-ray spectroscopy to identify and quantify specific gamma-emitting radionuclides.

Transuranic (TRU) waste is waste containing an alpha-emitting transuranic isotope (atomic number greater than 92) with a half-life greater than 20 years and containing more than 100 nanocuries per gram $(\mathrm{nCi} / \mathrm{g})$ of radioactivity. A combination of process knowledge and instrument measurement is used to determine if a waste is TRU waste. Waste in contact with TRU material in facility gloveboxes is automatically assumed to be TRU waste and handled accordingly. This waste is placed in five-gallon cans. The contents of the can are evaluated by a pulse height analyzer (PHA) that measures the various energy levels of gamma rays emitted by 
TRU wastes. The energy profile is used to determine the quantity of TRU material in the can. In almost every case, this material is determined to be TRU waste. Waste generated from maintenance activities outside the glovebox, which may contain TRU material, is handled as TRU waste if contamination surveys are greater than the procedural limit. The combination of process knowledge and instrument readings normally leads to a conservative determination.

\subsection{Waste Minimization/Pollution Prevention (WMin/P2)}

At the Savannah River Site (SRS) all operations are concerned with increasing Pollution Prevention (P2) awareness and implementation of improvement opportunities. Pollution prevention is integral to the SRS Environmental Management Policy, Environmental Management System (EMS), and Integrated Safety Management System (ISMS). SRS embraces pollution prevention as a primary strategy to operate in a compliant, cost-effective manner that protects the environment and the safety and health of employees and the public. SRS's P2 Program establishes the environmental management preference of source reduction and recycling over treatment, storage, and disposal and the preferred use of energy efficient and resource conservative practices and operations.

SRS P2 Program requirements are identified in the WSRC Environmental Compliance Manual (Procedure Manual 3Q), Procedure 6.11, "Pollution Prevention Program". The Waste Minimization/Pollution Prevention Program scope includes both in-field generator programs and site-wide coordination programs. The generator program is responsible for implementation of facility-specific improvement initiatives and is funded through each generator's operating budget. Site-wide program coordination which is managed by the Solid Waste Infrastructure organization is separately funded and provides the following:

- management support of Waste Minimization/Pollution Prevention Program

- technical assistance for facility walk-downs, lifecycle waste cost analyses, and pollution prevention opportunity assessments

- forums for waste minimization and P2 information and technology exchanges to support implementation of facility/activity-specific improvement initiatives

- increased employee P2 awareness and training programs

- contaminated metal and large equipment recycling and disposition

- mechanisms to increase waste generator accountability through the Solid Waste Management Council

- completion of required annual plans and reports

- implementations of site-wide initiatives such as sanitary waste recycle, Green-Is-Clean (GIC) programs, and other cost-cutting initiatives

- establishing P2 components in the Site's Communication Plan to increase public awareness and support

SRS encourages continuous improvement in its pollution prevention program for all our employees. Some key areas we addressed in FY2004 and are continuing through the end of the contract in FY2006 include:

- Continue to benchmark the best commercial industries and other DOE-managed facilities for pollution prevention and waste minimization activities.

- Continue to recover contaminated areas to reduce the risk to workers and the associated protective clothing, materials, and waste generated due to the existence of these areas.

- Continue to identify and use non-hazardous substitutes for hazardous products previously used on site. 
- Utilize the Energy Savings Performance contract to provide funding to install cost-effective energy conservation projects.

- Continue the retrofitting of administrative buildings with energy efficient lighting, occupancy sensors for lighting, and evaluation/upgrading of heating and ventilation systems for older buildings.

- Investigate and substitute recyclable and launderable products for those items that had previously been disposed.

- Continue the Presidential Closing the Circle Award-winning Chemical Commodity Management Center to control the purchase, distribution, and management of excess chemicals and chemical products.

- Procure cost-effective items made with recycled materials within the guidelines of the federal affirmative procurement practices to help in the development of the recyclables' market and to preserve our natural resources.

- Maintain funding dedicated to implementing cost-effective P2 projects and operations' improvements.

- Strengthen SRS practices and procedures to integrate pollution prevention evaluations into major process modifications and new facility and process designs.

- Maintain heightened employee awareness through training programs, video presentations, news articles, and employee involvement.

- Support public awareness of pollution prevention practices through public displays at offsite events and support of in-school teaching enrichment programs.

These efforts, when coupled with management support and direction that is driven through site-level program performance metrics, help ensure a sustainable and continuously improving SRS P2 Program. 
THIS PAGE INTENTIONALLY LEFT BLANK. 


\section{Chapter 3. Mixed Low-Level Waste Streams}

This chapter discusses the wastes, waste groups and treatment options for the mixed low-level waste stream category. The wastes are categorized according to the proposed treatment. These proposed treatment options include the following:

3.1 Mixed Low-Level Waste with Treatment Capacity

3.2 Mixed Low-Level Waste Streams Requiring Technology Development

3.3 Mixed Low-Level Waste Streams for Which Further Characterization is Required

3.4 Mixed Low-Level Waste Streams Requiring Radionuclide Decay Prior to LDR Treatment

3.5 SRS Treatment Facilities that have Discontinued Operations

Refer to Table 1.1, Chapter 1, of Volume II of the STP for the user's guide. The user's guide identifies each mixed waste stream, the preferred treatment option (PO), and the location where the waste stream is discussed in Volumes I and II of the STP.

\subsection{Mixed Low-Level Waste Streams with Treatment Capacity}

This section discusses the schedules and plans associated with treatment of SRS mixed waste with currently available treatment capacity. The sections of this chapter are divided into treatment categories as follows:

3.1.1 Onsite Treatment

3.1.2 Offsite Treatment

3.1.3 Preferred Treatment to be Determined

\subsubsection{Onsite Treatment}

SRS has several onsite treatment facilities available for treating mixed low level waste. These facilities include the F and H Effluent Treatment Project (F/H ETP) and the Saltstone facility. Also included in this section are treatability variance petition submittals, recycling, and miscellaneous onsite treatments not otherwise mentioned.

\subsubsection{F- and H-Area Effluent Treatment Project (F/H ETP)}

As waste characterizations are reviewed, aqueous characteristic waste, such as those in SR-W041 and SR-W077 may be treated at ETP if appropriate and approved. (See Section 9.1.1 of Volume II for more information.)

\section{SR-W077, Aqueous Characteristic Wastewater}

Certain volumes of this waste stream consist of certain aqueous sample residues and unused samples, particularly from groundwater monitoring wells, that have been returned from on-site or off-site laboratories. Laboratory waste in this stream also may include characteristically hazardous aqueous laboratory standards from onsite laboratories. Also included is purge water from wells at the Mixed Waste Management Facility. A variety of other wastewaters from various facilities contribute to this stream, e.g., wastewater collected from CIF sumps and found through analysis to be characteristically hazardous. Additional waste has been added to the original wastestream which requires treatment in the future.

Volume

- Volume Data for this waste stream can be found in Chapter 11.

Waste Stream Composition

- Aqueous wastewater 


\section{Waste Code}

- D002A (Corrosive wastewater)

- D005 (Barium)

- D006A (TCLP Cd)

- D007 (Chromium)

- D008A (TCLP Pb)

- D009A (TCLP Hg)

- D010 (Selenium)

- D011 (Silver)

- D018 (Benzene)

- D038 (Pyridine)

- D039 (Tetrachloroethylene)

- D040 (Trichloroethylene)

\section{LDR Treatment Standard}

- $\mathrm{D} 002 \mathrm{~A}=$ DEACT

- $\mathrm{D} 005=$ concentration based standard $=1.2 \mathrm{mg} / \mathrm{l}$

- $\mathrm{D} 006 \mathrm{~A}=$ concentration based standard $=0.69 \mathrm{mg} / \mathrm{l}$

- $\mathrm{D} 007=$ concentration based standard $=2.77 \mathrm{mg} / \mathrm{l}$

- $\mathrm{D} 008=$ concentration based standard $=0.69 \mathrm{mg} / \mathrm{l}$

- $\quad \mathrm{D} 009 \mathrm{~A}=$ concentration based standard $=0.15 \mathrm{mg} / \mathrm{l}$

- $\mathrm{D} 010=$ concentration based standard $=0.82 \mathrm{mg} / \mathrm{l}$

- $\mathrm{D} 011=$ concentration based standard $=0.43 \mathrm{mg} / \mathrm{l}$

- $\mathrm{D} 018=$ concentration based standard $=0.14 \mathrm{mg} / \mathrm{l}$

- $\mathrm{D} 038=$ concentration based standard $=0.14 \mathrm{mg} / \mathrm{l}$

- $\mathrm{D} 039=$ concentration based standard $=0.056 \mathrm{mg} / \mathrm{l}$

- $\mathrm{D} 040=$ concentration based standard $=0.054 \mathrm{mg} / \mathrm{l}$

D002A and D005-D011 wastewaters must also meet Universal Treatment Standards (UTS) for underlying hazardous constituents (UHCs) unless they are rendered non-hazardous and subsequently managed in a CWA treatment facility. D038, D039, and D040 wastewaters must also meet UTS for UHCs unless they are rendered nonhazardous and subsequently injected in a Class I SDWA well.

\section{Waste Characterization}

- Process knowledge and sample results

\section{Radiological Characterization}

- Alpha and beta/gamma emitters are present

\subsubsection{Reserved.}




\subsubsection{Onsite Treatment via Treatability Variance Petition}

Submittal of a treatability variance for macroencapsulation is the preferred option for certain mixed waste streams, including those described below.

\section{General Information}

The following waste streams are included in this waste group:

SR-W009, Silver Coated Packing Material

SR-W060, Tritiated Water with Mercury

Background Information

\section{SR-W009, Silver Coated Packing Material}

This material is ceramic packing material coated with silver nitrate (silver-coated Berl saddles) that is used in the offgas systems in the F-Canyon and H-Canyon dissolver operations to bond radioactive iodine ${ }^{129}$ and iodine ${ }^{131}$ emissions to the packing material as silver iodide. Spent packing material is changed out from the process when pluggage occurs or when the iodine level measured at the stack elevates such that levels start to approach the emission limit. Material is too small to meet the $60-\mathrm{mm}$ minimum particle size standard for debris.

Volume

- Volume data for this waste stream can be found in Chapter 11. The volume in Chapter 11 is reported as net volume. However, volume figures may be converted to gross in future annual updates once treatment is performed.

Waste Stream Composition

- Uncategorized inorganic particulate

Waste Code

- D011 (TCLP Ag)

- D009A (TCLP Hg)

- D008C (Radioactive lead solids subcategory)

- Nonwastewater

\section{LDR Treatment Standard}

- D008 = specified technology = Macroencapsulation

- D009 = concentration based standard $=0.025 \mathrm{mg} / \mathrm{l} \mathrm{TCLP}$

- $\mathrm{D} 011=$ concentration based standard $=0.14 \mathrm{mg} / \mathrm{l} \mathrm{TCLP}$

- D001 through D043 nonwastewaters to be land disposed must be treated to meet Universal Treatment Standards (UTS) for any underlying hazardous constituents (UHC) that may be present.

\section{Waste Characterization}

- No analysis was performed due to ALARA concerns, but silver value was calculated.

- Process knowledge is used to characterize waste stream.

- Confidence level for silver concentration is high due to manufacturer specifications of silver content on the saddles. Confidence level for mercury concentration is low; presence is suspected, but not proven. 
- Lead is included due to shielding in 55 gallon drums of F-Canyon waste.

\section{Radiological Characterization}

- Beta/gamma emitters are present.

- Volatile radionuclides $\mathrm{I}^{129}$ and $\mathrm{I}^{131}\left(\mathrm{I}^{131}\right.$ is a short lived isotope) are present.

- Typical rad levels include: $\mathrm{I}^{129}=62.2 \mathrm{nCi} / \mathrm{g}$

$\mathrm{Cs}^{137}=3080 \mathrm{nCi} / \mathrm{g}$

- Alpha emitters $\left(\mathrm{U}^{235}, \mathrm{U}^{236}, \mathrm{U}^{238}, \mathrm{Pu}^{239}\right.$, and $\left.\mathrm{Pu}^{240}\right)$ are present.

- Waste is remote handled.

- Mixed low-level waste

\section{SR-W060, Tritiated Water with Mercury}

This waste is highly tritiated heavy water with a small amount of mercury that has been adsorbed on silica gel. The waste was created by a spill incident resulting from a weld failure in a retired thermal diffusion column. The spill consisted of 17 liters of highly tritiated water and 3 or 4 milliliters of elemental mercury. The spill was absorbed with 50 kilograms of silica gel. The waste is contained in a welded stainless steel container, known colloquially as a "fat boy." There are no free liquids in this container.

Volume

- Data on the volume of this waste stream can be found in Chapter 11.

Waste Stream Composition

- Inorganic particulate

Waste Code

- D009A (TCLP Hg)

- Nonwastewater

LDR Treatment Standard

- $\mathrm{D} 009=$ concentration based standard $=0.025 \mathrm{mg} / \mathrm{l} \mathrm{TCLP}$

- D001 through D043 nonwastewaters to be land disposed must be treated to meet Universal Treatment Standards (UTS) for any underlying hazardous constituents (UHC) that may be present.

Waste Characterization

- Process knowledge is used to characterize the waste stream.

- Confidence level is medium.

Radiological Characterization

- 7,800 Ci of tritium

\subsubsection{Offsite Treatment}

For those waste streams that cannot be accommodated by SRS treatment facilities, various treatment technologies available at offsite facilities have been determined to be the preferred treatment option. This section discusses the treatment options that are available for offsite treatment. These include offsite vendor treatment and offsite treatment at other DOE facilities. 


\subsubsection{DOE Thermal Treatment}

The preferred options for the Polychlorinated Biphenyl (PCB) Mixed Waste Group are combustion at the ETTP TSCA Incinerator or vendor alternate debris technology treatment.

\section{General Information}

Currently, the sole representative of this waste group is:

SR-W079, Polychlorinated Biphenyl (PCB) Mixed Waste

\section{Background Information}

\section{SR-W079, Polychlorinated Biphenyl (PCB) Mixed Waste}

This waste stream has been reserved for all PCB-contaminated mixed waste for which treatment to destroy the PCBs is required under the Toxic Substances Control Act (TSCA) or debris that can be disposed under the PCB "mega rule" after RCRA contaminants are treated to meet LDR. This waste stream includes waste from laboratory analyses, paint chips, debris from the demolition of excess site facilities, and PCB spill clean-up waste.

\section{Volume}

- Volume data for this waste stream can be found in Chapter 11.

Waste Stream Composition

- PCB contaminated, organic liquid, miscellaneous solids

Waste Code

- D001B (Ignitable, high TOC nonwastewater)

- D002B (corrosive, nonwastewater)

- D004 (TCLP As)

- D005 (TCLP Ba)

- D006A (TCLP Cd)

- D007 (TCLP Cr)

- D008A (TCLP Pb)

- D009A (TCLP Hg)

- D010 (TCLP Se)

- D035 (Methyl ethyl ketone).

- D040 (Trichloroethylene).

- F001 and F002 (Spent halogenated solvents)

- Nonwastewater

LDR Treatment Standard

- $\mathrm{D} 001=$ specified technology $=$ CMBST

- $\mathrm{D} 002=$ specified technology $=$ DEACT

- D004 = concentration based standard $=5.0 \mathrm{mg} / \mathrm{l}$, TCLP

- $\mathrm{D} 005=$ concentration based standard $=21 \mathrm{mg} / \mathrm{l}, \mathrm{TCLP}$

- $\mathrm{D} 006=$ concentration based standard $=0.11 \mathrm{mg} / \mathrm{l}, \mathrm{TCLP}$

- $\mathrm{D} 007=$ concentration based standard $=0.60 \mathrm{mg} / \mathrm{l}, \mathrm{TCLP}$

- D008 $=$ concentration based standard $=0.75 \mathrm{mg} / \mathrm{l}$, TCLP

- D009 $=$ concentration based standard $=0.025 \mathrm{mg} / \mathrm{l}$, TCLP

- $\mathrm{D} 010=$ concentration based standard $=5.7 \mathrm{mg} / \mathrm{l}, \mathrm{TCLP}$

- D035 = concentration based standard $=36 \mathrm{mg} / \mathrm{kg}$. 
- $\mathrm{D} 040=$ concentration based standard $=6.0 \mathrm{mg} / \mathrm{kg}$.

- F001 and F002 = concentration based standard $=6-30 \mathrm{mg} / \mathrm{kg}$

- D001 through D043 nonwastewaters to be land disposed must be treated to meet Universal Treatment Standards (UTS) for any underlying hazardous constituents (UHC) that may be present.

Waste Characterization

- Sampling and analysis are used to characterize this waste stream.

- Confidence level is high because sampling and analysis has been performed.

\section{Radiological Characterization}

- Beta/gamma emitters are present $\left(\mathrm{Cs}^{137}, \mathrm{Sr}^{90}\right.$, and others).

- Alpha emitters are present $\left(\mathrm{U}^{235}, \mathrm{U}^{238}, \mathrm{Pu}^{239}, \mathrm{Pu}^{240}\right.$, and others).

- Contact handled

- Mixed low-level waste

\subsubsection{Commercial Vendor Debris Treatment}

Alternate debris technology treatment, macroencapsulation, shredding followed by stabilization, at an offsite vendor's facility are the preferred options for certain mixed waste streams, including, the following:

SR-W062, Low-Level Contaminated Debris

\section{General Information}

\section{SR-W062, Low-Level Contaminated Debris}

This waste stream consists of debris (metal, floor tiles, fluorescent light bulbs, instruments, and other equipment including debris generated from operations at CIF and machinery used in the remediation of various contamination sites that could not be decontaminated) contaminated with TCLP metals and radionuclides. (NOTE: this is a different stream from SR-W015, Mercury/Tritium Contaminated Equipment). This waste requires a permitted TSD for treatment since it has been in permitted storage.

Also included in this waste stream are tools and other non-incinerable items found in waste stream SR-W055, Job Control Waste Containing Solvent Contaminated Wipes and PPE, SR-W025, Solvent/TRU Job Control Waste < $100 \mathrm{nCi} / \mathrm{g}$; SR-W042, Paints and Thinners; and other waste streams shredded in preparation for treatment by combustion.

In addition to the CIF debris, this waste stream also consists of filters, job control waste from the segregation and decontamination of lead in Building 105-C, and soil associated with other equipment or debris from the M-Area Vendor Treatment Facility, the tank farms, and other site locations. Radioactive $\mathrm{Hg} / \mathrm{Ni}-\mathrm{Cad}$ batteries have recently been approved by EPA for macroencapsulation and are therefore included in this wastestream.

Volume

- Volume data on this waste stream can be found in Chapter 11.

Waste Stream Composition

- Inorganic debris

Waste Code 
- D004 (TCLP As)

- D005 (TCLP Ba)

- D006A (TCLP Cd)

- D007 (TCLP Cr)

- D008A (TCLP Pb)

- D009A (TCLP Hg)

- D010 (TCLP Se)

- D011 (TCLP Ag)

- D035 (Methyl ethyl ketone)

- D039 (Tetrachloroethylene)

- F001 (Unspecified solvents)

- F002 (Unspecified solvents)

- F003X (Unspecified solvents)

- F004X (Unspecified solvents)

- F005X (Unspecified solvents)

- F006 (Metal plating waste without cyanide)

- P048 (2,4-Dinitrophenol)

- P113 (Thallic Oxide)

- U002 (Acetone)

- U003 (Acetonitrile)

- U019 (Benzene)

- U037 (Chlorobenzene)

- U080 (Methylene Chloride)

- U123 (Formic Acid)

- U127 (Hexachlorobenzene)

- U131 (Hexachloroethane)

- U151 (TCLP Hg)

- U159 (Methyl Ethyl Ketone)

- U165 (Naphthalene)

- U188 (Phenol)

- U209 (1,1,2,2-Tetrachloroethane)

- U210 (Tetrachloroethylene)

- U211 (Carbon Tetrachloride)

- U220 (Toluene)

- U226 (1,1,1-Trichloroethane)

- U228 (Trichloroethylene)

- U239 (Xylenes)

- Nonwastewater 
Specific waste codes will vary depending upon where the waste was generated. Wastes from CIF contain all of the waste codes that are fed to CIF. The F-listed solvents listed reflect the inclusion of non-incinerable items found in waste streams SR-W025 and SR-W055. Items from SR-W025 and SR-W055 may contain other P- and U-listed codes.

\section{LDR Treatment Standard}

- D004 $=$ concentration based standard $=5.0 \mathrm{mg} / \mathrm{l}, \mathrm{TCLP}$

- D005 = concentration based standard $=21 \mathrm{mg} / \mathrm{l}, \mathrm{TCLP}$

- $\mathrm{D} 006=$ concentration based standard $=0.11 \mathrm{mg} / \mathrm{l}, \mathrm{TCLP}$

- D007 $=$ concentration based standard $=0.60 \mathrm{mg} / \mathrm{l}$, TCLP

- $\mathrm{D} 008=$ concentration based standard $=0.75 \mathrm{mg} / \mathrm{l}$, TCLP

- D009 $=$ concentration based standard $=0.025 \mathrm{mg} / \mathrm{l}$, TCLP

- D010 = concentration based standard $=5.7 \mathrm{mg} / \mathrm{l}, \mathrm{TCLP}$

- $\mathrm{D} 011=$ concentration based standard $=0.14 \mathrm{mg} / \mathrm{l}, \mathrm{TCLP}$

- D035 = concentration based standard $=36 \mathrm{mg} / \mathrm{kg}$

- $\mathrm{D} 039=$ concentration based standard $=0.14 \mathrm{mg} / \mathrm{l}, \mathrm{TCLP}$

- $\mathrm{F} 001=$ concentration based standard $=6-30 \mathrm{mg} / \mathrm{kg}$

- $\mathrm{F} 002=$ concentration based standard $=6-30 \mathrm{mg} / \mathrm{kg}$

- $\mathrm{F} 003=$ concentration based standard $=2.6-60 \mathrm{mg} / \mathrm{kg}$

- F004 = concentration based standard $=6-160 \mathrm{mg} / \mathrm{kg}$

- F005 $=$ concentration based standard $=10-170 \mathrm{mg} / \mathrm{kg}$

- F006 $=$ concentration based standard $=0.19-5.0 \mathrm{mg} / \mathrm{l}$, TCLP

- $\mathrm{P} 048=$ concentration based standard $=160 \mathrm{mg} / \mathrm{kg}$

- P113 = specified technology = RTHRM; or STABL

- $\mathrm{U} 002=$ concentration based standard $=160 \mathrm{mg} / \mathrm{kg}$

- $\mathrm{U} 003=$ specified technology $=$ CMBST

- $\mathrm{U} 019=$ concentration based standard $=10 \mathrm{mg} / \mathrm{kg}$

- $\mathrm{U} 037=$ concentration based standard $=6 \mathrm{mg} / \mathrm{kg}$

- $\mathrm{U} 080=$ concentration based standard $=30 \mathrm{mg} / \mathrm{kg}$

- $\mathrm{U} 123=$ specified technology $=$ CMBST

- $\mathrm{U} 127=$ concentration based standard $=10 \mathrm{mg} / \mathrm{kg}$

- $\mathrm{U} 131=$ concentration based standard $=30 \mathrm{mg} / \mathrm{kg}$

- $\mathrm{U} 151=$ concentration based standard $=0.025 \mathrm{mg} / \mathrm{l}$, TCLP

- $\mathrm{U} 159=$ concentration based standard $=36 \mathrm{mg} / \mathrm{kg}$

- $\mathrm{U} 165=$ concentration based standard $=5.6 \mathrm{mg} / \mathrm{kg}$

- $\mathrm{U} 188=$ concentration based standard $=6.2 \mathrm{mg} / \mathrm{kg}$

- $\mathrm{U} 209=$ concentration based standard $=6.0 \mathrm{mg} / \mathrm{kg}$

- $\mathrm{U} 210=$ concentration based standard $=6 \mathrm{mg} / \mathrm{kg}$

- $\mathrm{U} 211=$ concentration based standard $=6 \mathrm{mg} / \mathrm{kg}$

- $\mathrm{U} 220=$ concentration based standard $=10 \mathrm{mg} / \mathrm{kg}$

- $\mathrm{U} 226=$ concentration based standard $=6 \mathrm{mg} / \mathrm{kg}$

- $\mathrm{U} 228=$ concentration based standard $=6.0 \mathrm{mg} / \mathrm{kg}$

- $\mathrm{U} 239=$ concentration based standard $=30 \mathrm{mg} / \mathrm{kg}$

- D001 through D043 nonwastewaters to be land disposed must be treated to meet Universal Treatment Standards (UTS) for any underlying hazardous constituents (UHC) that may be present.

Waste debris from CIF will have treatment standards that are reflected in the waste fed to CIF. Specific information on treatment standards can be acquired by looking at specific wastes in Volume II.

\section{Waste Characterization}

- Process knowledge is used to characterize the waste stream.

- Confidence level is high based on knowing process history of the waste. 


\section{Radiological Characterization}

- Radioactivity will vary depending on the generation source and location.

- Waste is contact handled.

- Mixed low-level waste

\subsubsection{Commercial Vendor Lead Treatment}

Lead Macroencapsulation at an offsite vendor's facility is the preferred option for certain of the commercial vendor or is readily handled by the commercial vendor under special procedures.

\section{SR-W069, Low-Level Waste (LLW) Lead-to be Macroencapsulated Offsite}

This waste stream consists of low-level waste lead and lead compounds that are inseparably mixed with non-lead components. Examples of this waste stream are lead-lined gloves, aprons and equipment containing lead solder, and radioactive lead acid batteries. This stream also includes lead shielding, bricks, etc., that cannot be decontaminated.

Volume

- Volume data for this waste stream can be found in Chapter 11.

Waste Stream Composition

- Elemental lead

- Non-elemental lead

Waste Code

- D002 (corrosive)

- D004 (TCLP As)

- D006 (TCLP Cd)

- D008A (TCLP Pb)

- D008B (lead acid batteries and radioactive lead solids)

- D008C (elemental Pb)

- Nonwastewater

LDR Treatment Standard

- $\mathrm{D} 008=$ concentration based technology $=0.75 \mathrm{mg} / \mathrm{l} \mathrm{TCLP}$; or specified technology $=$ MACRO for radioactive elemental lead

- D008 specified technology

\section{Waste Characterization}

- Process knowledge is used to characterize the waste stream.

- Confidence level is high based on the fact that waste is easily identified as containing lead.

Radiological Characterization

- Beta/gamma emitters $\left(\mathrm{Cs}^{137}\right.$ and $\left.\mathrm{Sr}^{90}\right)$ are present.

- Alpha emitters $\left(\mathrm{Pu}^{238}, \mathrm{Pu}^{239}\right.$, and $\left.\mathrm{U}^{235}\right)$ are present.

- Waste is contact handled.

- Mixed low-level waste 


\subsubsection{Commercial Vendor Mercury Treatment}

The preferred option is direct amalgamation (AMLGM)or stabilization by a commercial vendor, dependent upon vendor states' approval of direct AMLGM for the waste. The following wastestreams are in this category:

SR-W014, Elemental (Liquid) Mercury - Sitewide

SR-W086, Characteristically Hazardous Non-Incinerable Solids

\section{General Information}

\section{SR-W014, Elemental(Liquid) Mercury - Sitewide}

This waste stream includes elemental mercury generated at different SRS facilities, primarily at the HLW Tank Farms, DWPF and Defense Programs. At the HLW Tank Farms and DWPF, elemental mercury is recovered during the evaporation and vitrification of high-level waste. Currently, H-Canyon Dissolving Operations will re-use a portion of the mercury generated by the HLW Tank Farms and DWPF. The re-use will continue until the dissolving operations cease. This waste stream also includes elemental mercury used as a pumping fluid in diffusion pumps for the transfer of tritium gas. The mercury waste is generated from pump maintenance or pump failure due to mercury oxide fouling. This waste stream also includes broken thermometers, unbroken thermometers and mercury spill clean up materials generated sitewide.

Volume

- Volume data for this waste stream can be found in Chapter 11.

Waste Stream Composition

- Elemental mercury

Waste Code

- D009D (elemental mercury)

- Nonwastewater

LDR Treatment Standard

- $\mathrm{D} 009=$ specified technology $=$ AMLGM

Waste Characterization

- Process knowledge is used to characterize the waste stream.

- Confidence level is high based on the waste composition.

\section{Radiological Characterization}

- Radioactivity will vary depending on the generation source and location.

- Waste is contact handled and meets NRC Class A limits.

- Mixed low-level waste.

\section{SR-W068, Elemental (Liquid) Mercury - Sitewide}

This waste stream is elemental mercury generated at different SRS facilities, primarily at DWPF and the HLW Tank Farms. At DWPF, elemental mercury is recovered during the vitrification of high-level waste. Elemental mercury is recovered during the evaporation of high-level waste in the HLW Tank Farms. Currently, H-Canyon Dissolving Operations will re-use a portion of the mercury generated by DWPF and the HLW Tank Farms. The re-use will 
continue until the dissolving operations cease. Some portion of the mercury generated may be managed as waste stream SR-W068 and will be consolidated with SR-W014.

Volume

- Volume data for this waste stream can be found in Chapter 11.

Waste Stream Composition

- Elemental mercury

Waste Code

- D009D (elemental mercury)

- Nonwastewater

LDR Treatment Standard

- $\mathrm{D} 009 \mathrm{D}=$ specified technology $=$ AMLGM

Waste Characterization

- Process knowledge is used to characterize the waste stream.

- Confidence level is high based on the waste composition.

\section{Radiological Characterization}

- Radioactivity will vary depending on the generation source and location.

- Waste is contact handled.

- Mixed low-level waste

\section{SR-W086, Characteristically Hazardous Non-Incinerable Solids}

This waste stream is filters, swipes and other cleanup material that contains high mercury concentrations greater than $260 \mathrm{mg} / \mathrm{kg}$. The waste generated from mercury spill cleanup, broken and unbroken thermometers and filtering activities. This waste stream also includes batteries, floor tiles and fluorescent light bulbs.

Volume

- Volume data for this waste stream can be found in Chapter 11.

Waste Stream Composition

- Heterogeneous organic and inorganic debris

Waste Code

- D007 TCLP (chromium)

- D009C High mercury (inorganic)

- D009D Elemental mercury

- D008 TCLP (lead)

LDR Treatment Standard

- RMERC

- AMLGM 


\section{Waste Characterization}

- Sampling and analysis were used to characterize this waste stream.

- Confidence is high.

\section{Radiological Characterization}

- Average total activity is $50 \mathrm{nCi} / \mathrm{g}$ with depleted uranium.

- Waste is contact handled.

- Mixed low-level waste

\subsubsection{Commercial Vendor Solids Treatment}

The preferred treatment option can include several treatment steps. Wastes that contain organic material in excess of the LDR Treatment Standard are initially treated to separate the organic portion of the contaminants using solvent extraction or thermal separation. The desorbed chemical constituents are then treated via direct chemical oxidation or combustion. The waste material remaining after organics or non-organic waste material are removed is then either directly stabilized, shredded and stabilized, macroencapsulated or subjected to alternative debris treatment technologies. No stabilization of organics is performed, and no impermissible dilution occurs. Wastes that do not contain significant organics are either directly stabilized, shredded and stabilized, macroencapsulated or subjected to alternative debris treatment technologies. as appropriate under RCRA regulations.

The following waste streams are included in this category:

SR-W048, Soils from Spill Remediation (stabilization thermal treatment)

SR-W073, Cadmium-containing Raschig Rings (shredded and stabilized)

SR-W082, Radioactive CMP Soils (stabilization thermal treatment)

SR-W088, Sludges and Non-Debris Solids (thermal treatment, stabilization)

\section{SR-W048, Soils from Spill Remediation}

This waste consists of soils, sand, and associated debris (rocks, wood, etc.) resulting from cleanup activities of spills surrounding operations. This waste stream does not include any soils to be addressed in the Environmental Restoration program. The original value of waste in stream SR-W048 was treated in M-Area VTF. Additional waste has now been added to SR-W048 with a new preferred option.

Volume

- Volume data for this waste stream can be found in Chapter 11.

Waste Stream Composition 
- Uncategorized soils

Waste Code

- D002 (corrosive)

- D006 (TCLP Cd)

- D007 (TCLP Cr)

- D008 (TCLP Pb)

- D009A (TCLP Hg)

- F001 (spent halogenated degreasing solvents)

- Nonwastewater

LDR Treatment Standard

- $\mathrm{D} 002=\mathrm{DEACT}$

- $\mathrm{D} 006=$ concentration based standard $=0.11 \mathrm{mg} / \mathrm{l}$, TCLP

- $\mathrm{D} 007=$ concentration based standard $=0.60 \mathrm{mg} / \mathrm{l}$, TCLP

- D008 $=$ concentration based standard $=0.75 \mathrm{mg} / \mathrm{l}$, TCLP

- D009 = concentration based standard $=0.02 \mathrm{mg} / \mathrm{l}$, TCLP

- $\mathrm{F} 001=$ concentration based standard $=6-30 \mathrm{mg} / \mathrm{kg}$

Waste Characterization

- Process knowledge is used to characterize the waste stream.

- Confidence level is high based on process knowledge of what was spilled or located at a particular site.

Radiological Characterization

- Beta/gamma and alpha emitters are present.

- Waste is contact handled.

- Mixed low-level waste.

\section{SR-W073, Cadmium-Containing Raschig Rings}

This waste stream is composed of approximately $60 \%$ rubbery-plastic material, $20 \%$ lead, and $20 \%$ cadmium (by volume). These Raschig Rings were used as a criticality prevention measure in certain sumps in the Separations $\mathrm{H}$ Area facility. Waste size is less than the $60 \mathrm{~mm}$ minimum particle standard for debris.

Volume

- Volume data for this waste stream can be found in Chapter 11.

Waste Stream Composition

- Other organic particulates

Waste Codes

- D006A (TCLP Cd)

- Nonwastewater

LDR Treatment Standard

- $\mathrm{D} 006=$ concentration based standard $=0.11 \mathrm{mg} / \mathrm{l}$ TCLP

- D008 $=$ concentration based standard $=0.75 \mathrm{mg} / \mathrm{l} \mathrm{TCLP}$. (UTS) 


\section{Waste Characterization}

- TCLP tests were performed to verify hazardous characteristic.

\section{Radiological Characterization}

- Radioactive contamination for alpha and beta/gamma was detected in analysis.

- Material was generated in a contamination area.

\section{SR-W082, Radioactive Chemicals, Metals, and Pesticides (CMP) Soils}

This waste stream is composed of soil and debris containing spent solvents, metal contaminants and tetrachloroethylene removed from the organic and metal portions of the CMP disposal pits. This soil was excavated during a RCRA closure of the site.

Volume

- Volume data for this waste stream can be found in Chapter 11.

Waste Codes

- $\quad$ D004 (TCLP As)

- D006 (TCLP Cd)

- D007 (TCLP Cr)

- D008A (TCLP Pb)

- D010 (TCLP Se)

- D039 (TCLP tetrachloroethylene)

- F001 (trichloroethylene, methylene chloride)

- F002 (trichloroethylene, methylene chloride)

- U210 (tetrachloroethylene)

- Nonwastewater

LDR Treatment Standard

- $\quad$ D004 $=$ Concentration Based Standard $=5.0 \mathrm{mg} / 1 \mathrm{TCLP}$

- $\mathrm{D} 006=$ Concentration Based Standard $=0.11 \mathrm{mg} / 1 \mathrm{TCLP}$

- $\mathrm{D} 007=$ Concentration Based Standard $=0.60 \mathrm{mg} / 1 \mathrm{TCLP}$

- $\quad$ D008 $=$ Concentration Based Standard $=0.75 \mathrm{mg} / 1$ TCLP

- $\mathrm{D} 010=$ Concentration Based Standard $=5.7 \mathrm{mg} / 1 \mathrm{TCLP}$

- $\mathrm{D} 039=$ Concentration Based Standard $=6.0 \mathrm{mg} / 1$ Total

- $\quad$ F001 $=$ Concentration Based Standard $=6.0 \mathrm{mg} / \mathrm{kg}$ Total (tetrachloroethylene); $30 \mathrm{mg} / \mathrm{kg}$ total (methylene chloride)

- $\quad \mathrm{F} 002=$ Concentration Based Standard $=6.0 \mathrm{mg} / \mathrm{kg}$ Total (tetrachloroethylene) $; 30 \mathrm{mg} / \mathrm{kg}$ total (methylene chloride)

- $\mathrm{U} 210=$ Concentration Based Standard $=6.0 \mathrm{mg} / \mathrm{kg}$ Total

- D001 through D043 nonwastewaters to be land disposed must be treated to meet Universal Treatment Standards (UTS) for any underlying hazardous constituents (UHC) that may be present.

Waste Characterization

- Process knowledge 


\section{Radiological Characterization}

- Tritium is the only isotope known to be present.

- Contact handled.

\section{SR-W088, Sludges and Non-Debris Solids}

This waste stream consists primarily of dry solid material such as scabbled concrete, paint chips and crushed light bulbs. The scabbled concrete is generated from the demolition of SRS facilities performed by Site D\&D (SDD). Paint chips and spent contaminated light bulbs are generated by sitewide maintenance activities. These waste forms are primarily hazardous for metals. This stream also includes halogenated or non-halogenated sludges that require stabilization

Volume

- Volume data for this waste stream can be found in Chapter 11.

Waste Codes

- D004 (TCLP As)

- D005 (TCLP Ba)

- D006A (TCLP Cd)

- D007 (TCLP Cr)

- D008A (TCLP Pb)

- D009A (TCLP Hg)

- D010 (TCLP Se)

- D011 (TCLP Ag)

- Nonwastewater

\section{LDR Treatment Standard}

- $\mathrm{D} 004=$ concentration based standard $=5.0 \mathrm{mg} / \mathrm{l}, \mathrm{TCLP}$

- D005 = concentration based standard $=21 \mathrm{mg} / \mathrm{l}, \mathrm{TCLP}$

- D006 $=$ concentration based standard $=0.11 \mathrm{mg} / \mathrm{l}, \mathrm{TCLP}$

- D007 $=$ concentration based standard $=0.60 \mathrm{mg} / \mathrm{l}, \mathrm{TCLP}$

- D008 $=$ concentration based standard $=0.75 \mathrm{mg} / \mathrm{l}, \mathrm{TCLP}$

- $\mathrm{D} 009=$ concentration based standard $=0.025 \mathrm{mg} / \mathrm{l}$, TCLP

- $\mathrm{D} 010=$ concentration based standard $=5.7 \mathrm{mg} / \mathrm{l}, \mathrm{TCLP}$

- D011 = concentration based standard $=0.14 \mathrm{mg} / \mathrm{l}$, TCLP

Waste Characterization

- Process knowledge and sampling and analysis have been used to characterize these waste streams.

Radiological Characterization

- Alpha and beta/gamma emitters are present.

- Contact handled.

\subsubsection{Commercial Vendor "Non-PUREX” Treatment}

The preferred treatment option can include several treatment steps. Wastes that contain organic material in excess of the LDR Treatment Standard are initially treated to separate the organic portion of the contaminants using solvent 
extraction or thermal separation. The desorbed chemical constituents are then treated via direct chemical oxidation or thermal treatment. The waste material remaining after organics are removed is then either directly stabilized, shredded and stabilized, macroencapsulated or subjected to alternative debris treatment technologies. No stabilization of organics is performed, and no impermissible dilution occurs.

The following waste streams are included in this category:

SR-W001, Rad-Contaminated Solvents (thermal treatment)

SR-W003, Solvent Contaminated Debris (LLW) (macroencapsulation or stabilization)

SR-W012, Toxic Characteristic Solids (macroencapsulation or stabilization)

SR-W018, Listed Incinerable Solids (macroencapsulation or stabilization)

SR-W035, Mixed Waste Oil-Sitewide (thermal treatment (aqueous portion stabilized))

SR-W042, Paints and Thinners (thermal treatment or stabilization)

SR-W051, Spent Filter Cartridges and Carbon Filter Media (macroencapsulation or stabilization)

SR-W055, Job Control Waste Containing Solvent Contaminated Wipes (macroencapsulation or stabilization)

SR-W070, Mixed Waste from Laboratory Samples (thermal treatment or stabilization)

SR-W071, Wastewater Suitable for Thermal Treatment or Stabilization (Broad Spectrum)

SR-W080, Charleston Naval Shipyard Waste (CN-W001, CN-W004)

SR-W081, Reactive/Ignitable Waste*

SR-W087, Bettis Atomic Power Laboratory Contaminated Oil, (BT-W035 part of BT-W003)

*SR-W081 has undergone deactivation at generator facility prior to final treatment by commercial vendor.

\section{SR-W001, Rad-Contaminated Solvents:}

This waste stream is radioactively contaminated solvent and solvent mixtures such as solvents used for cleaning equipment in the Separations or Reactors Areas, degreasing solvents for depleted uranium fines used to assure unhindered adsorption of water in the tritium process, used or unused organic reagents and solutions used in bioassay and other laboratory analyses, and catalyst material for an incinerator that is no longer operational. The non-halogenated solvents in storage are wastes that used carbon $\left(\mathrm{C}^{14}\right)$ and tritium $\left(\mathrm{H}^{3}\right)$ labeled materials as tracers, or mixtures of waste scintillation counter calibration standards. The halogenated solvents are degreasing solvents contaminated with tritium and uranium. This waste stream is a consolidation of SR-W001, Rad-Contaminated Solvents; SR-W002, Rad-Contaminated Chlorofluorocarbons; SR-W010, Scintillation Solution; SR-W030, Spent Methanol Solution; and SR-W059, Tetrabutyl Titanate. Added to the waste stream will be solvent waste generated from CIF operations. This waste stream also includes liquids formerly in waste stream SR-W078, Hazardous Waste Awaiting Radiological Screening (stream now closed), which the initial results of radiological analysis have shown to be mixed waste, or are indeterminate.

Volume

- Volume data for this waste stream can be found in Chapter 11.

Waste Stream Composition

- Organic liquid 
Waste Code

- D001A (ignitable high TOC)

- D002 (corrosive, nonwastewater

- D006A (TCLP Cd)

- D007 (TCLP Cr)

- D008A (TCLP Pb)

- D009 (TCLP Hg)

- D010 (TCLP Se)

- D011 (TCLP Ag)

- D018 (Benzene)

- D019 (Carbon tetrachloride)

- D022 (Chloroform)

- F001, F002, F003, F005A (halogenated and nonhalogenated spent solvents)

- F006 (wastewater treatment sludges from electroplating operations)

- U002 (Acetone)

- U003 (Acetonitrite)

- U019 (Benzene)

- U037 (Chlorobenzene)

- U045 (Methane, Chloro-(I,T) methyl chloride)

- U080 (Methylene chloride)

- U108 (1,4-Dioxane)

- U123 (Formic acid)

- U127 (Hexachlorobenzene)

- U131 (Hexachloroehtane)

- U159 (Methyl ethyl ketone)

- U165 (Napthalene)

- U188 (Phenol)

- U210 (Tetrachloroethylene)

- U211 (Carbon tetrachloride)

- U220 (Toluene)

- U226 (1,1,1-Trichloroethane)

- U228 (Trichloroethylene)

- U239 (Xylene)

- Nonwastewater

Since this waste stream will include wastes generated by previous CIF operations, additional waste codes may apply to this stream. Waste codes will depend on the specific generation episode at CIF. Potentially, any of the many waste codes included in the CIF RCRA Part B permit could apply.

\section{LDR Treatment Standard}

- D001 $=$ specified technology $=$ RORGS or CMBST

- D002 = specified technology $=$ DEACT

- $\mathrm{D} 006=$ concentration based standard $=0.11 \mathrm{mg} / \mathrm{l} \mathrm{TCLP}$

- $\mathrm{D} 007=$ concentration based standard $=0.60 \mathrm{mg} / \mathrm{l} \mathrm{TCLP}$

- D008 $=$ concentration based standard $=0.75 \mathrm{mg} / \mathrm{l} \mathrm{TCLP}$

- D009 $=$ concentration based standard $=0.025 \mathrm{mg} / \mathrm{l}$ TCLP

- D010 = concentration based standard $=5.7 \mathrm{mg} / \mathrm{l} \mathrm{TCLP}$

- $\mathrm{D} 011=$ concentration based standard $=0.14 \mathrm{mg} / \mathrm{l}$ TCLP

- D018 = concentration based standard $=10 \mathrm{mg} / \mathrm{kg}$, UTS $=10 \mathrm{mg} / \mathrm{kg}$

- D019 and D022 = concentration based standard $=6.0 \mathrm{mg} / \mathrm{kg}, \mathrm{UTS}=6.0 \mathrm{mg} / \mathrm{kg}$

- F001 and F002 = concentration based standard $=6.0-30 \mathrm{mg} / \mathrm{kg}$

- $\mathrm{F} 003=$ concentration based standard $=0.75 \mathrm{mg} / \mathrm{l}$, TCLP $-160 \mathrm{mg} / \mathrm{kg}$

- $\mathrm{F} 005=$ concentration based standard $=4.8 \mathrm{mg} / \mathrm{l}$, TCLP-170 $\mathrm{mg} / \mathrm{kg}$ except 2-Ethoxyethanol and 2-Nitropropane = CMBST

- $\mathrm{F} 006=$ concentration based standard $=0.11-11.0 \mathrm{mg} / \mathrm{l} \mathrm{TCLP}$ 
- $\mathrm{U} 002=$ concentration based standard $=160 \mathrm{mg} / \mathrm{kg}$

- $\mathrm{U} 003=$ concentration based standard $=38 \mathrm{mg} / \mathrm{kg}$ or CMBST

- $\mathrm{U} 019=$ concentration based standard $=10 \mathrm{mg} / \mathrm{kg}$

- $\mathrm{U} 037=$ concentration based standard $=6.0 \mathrm{mg} / \mathrm{kg}$

- $\mathrm{U} 045=$ concentration based standard $=30.0 \mathrm{mg} / \mathrm{kg}$

- $\mathrm{U} 080=$ concentration based standard $=30 \mathrm{mg} / \mathrm{kg}$

- $\mathrm{U} 108=$ specified technology $=$ CMBST

- $\mathrm{U} 123=$ specified technology $=$ CMBST

- $\mathrm{U} 127=$ concentration based standard $=10 \mathrm{mg} / \mathrm{kg}$

- $\mathrm{U} 131=$ concentration based standard $=30 \mathrm{mg} / \mathrm{kg}$

- $\mathrm{U} 159=$ concentration based standard $=36 \mathrm{mg} / \mathrm{kg}$

- $\mathrm{U} 165=$ concentration based standard $=5.6 \mathrm{mg} / \mathrm{kg}$

- $\mathrm{U} 188=$ concentration based standard $=6.2 \mathrm{mg} / \mathrm{kg}$

- $\mathrm{U} 210=$ concentration based standard $=6.0 \mathrm{mg} / \mathrm{kg}$

- $\mathrm{U} 211=$ concentration based standard $=6.0 \mathrm{mg} / \mathrm{kg}$

- $\mathrm{U} 220=$ concentration based standard $=10.0 \mathrm{mg} / \mathrm{kg}$

- $\mathrm{U} 226=$ concentration based standard $=6.0 \mathrm{mg} / \mathrm{kg}$

- $\mathrm{U} 228=$ concentration based standard $=6.0 \mathrm{mg} / \mathrm{kg}$

- $\mathrm{U} 239=$ concentration based standard $=30 \mathrm{mg} / \mathrm{kg}$

- D001 through D043 nonwastewaters to be land disposed must be treated to meet Universal Treatment Standards (UTS) for any underlying hazardous constituents (UHC) that may be present.

\section{Waste Characterization}

- Process knowledge and sampling and analysis have been used to characterize waste streams.

- Confidence level is high based upon the known composition of the solvents used in the processes and of sample analyses for some of the organics.

\section{Radiological Characterization}

- Sampling and analysis results indicate tritium present up to $1700 \mu \mathrm{Ci} / \mathrm{ml}$.

- Beta/gamma emitters

- $\mathrm{U}^{238}$ alpha present is in solvent from the tritium facility and Reactor Materials Facilities.

- Alpha $\left(\mathrm{U}^{235}, \mathrm{U}^{238}, \mathrm{Pu}^{238}, \mathrm{Pu}^{239}\right)$ emitters are present.

- Waste is contact handled.

- Mixed low-level waste

\section{SR-W003, Solvent Contaminated Debris (LLW)}

The stream is a collection of similar debris whose LDR treatment standards can be met by combustion. The waste stream includes spent solvent contaminated rags and wipes generated sitewide in the clean up of interior spills and for decontamination. This waste stream also includes job control waste and other incinerable debris. The waste codes indicate the components that may be present in the waste stream as a whole. Waste codes listed in the waste stream would vary depending on where the waste came from within SRS.

\section{Volume}

- Volume data for this waste stream can be found in Chapter 11.

Waste Stream Composition

- Organic debris

Waste Code

- D004 (TCLP As)

- D005 (TCLP Ba) 
- D006A (TCLP Cd)

- D008A (TCLP Pb)

- D010 (TCLP Se)

- D012 (Endrin)

- D014 (Methoxychlor)

- D016 (2,4,-D)

- D018 (Benzene)

- D020 (Chlordane)

- D022 (Chloroform)

- D024 (m-Cresol)

- D026 (Total Cresols)

- D028 (1,2-Dichloroethane)

- D030 (2,4-Dinitrotoluene)

- D032 (Hexachlorobenzene)

- D034 (Hexachloroethane)

- D036 (Nitrobenzene)

- D038 (Pyridine)

- D040 (Trichloroethylene)

- D042 (2,4,6-Trichlorophenol)

- F001 (Spent halogenated degreasing solvents)

- F003A (Spent nonhalogenated solvents)

- F006 (wastewater treatment sludges from electrop

- U002 (Acetone)

- U019 (Benzene)

- U080 (Methylene chloride)

- U127 (Hexachlorobenzene)

- U159 (Methyl Ethyl Ketone)

- U188 (Phenol)

- U211 (Carbon tetrachloride)

- U226 (1,1,1-Trichloroethane)

- U239 (Xylene)
- D007 (TCLP Cr)

- D009A (TCLP Hg)

- D011 (TCLP Ag)

- D013 (Lindane)

- D015 (Toxaphene)

- D017 (2, 4, 5-TP)

- D019 (Carbon tetrachloride)

- D021 (Chlorobenzene)

- D023 (o-Cresol

- D025 (p-Cresol)

- D027 (p-Dichlorobenzene)

- D029 (1.1-Dichloroethylene)

- D031 (Heptachlor)

- D033 (Hexachlorobutadiene

- D035 (Methyl ethyl ketone)

- D037 (Pentachlorophenol)

- D039 (Tetrachloroethylene)

- D041 (2,4,5-Trichlorophenol)

- D043 (Vinyl chloride)

- F002 (Spent halogenated solvents)

- F005A (Halogenated and nonhalogenated spent solvents)

plating operations)

- U003 (Acetonitrile)

- U037 (Chlorobenzene)

- U123 (Formic acid)

- U131 (Hexachloroethane)

- U165 (Naphthalene)

- U210 (Tetrachloroethylene)

- U220 (Toluene)

- U228 (Trichloroethylene)

- Nonwastewater

Since this waste stream could include solvent contaminated rags and wipes from spill clean-ups at CIF, waste codes could include any of the wastes treated at CIF. Refer to the waste code lists for SR-W046 and SR-W047 for all of the waste codes possible.

For that portion of waste stream SR-W003 generated from other locations at SRS, waste codes include D004-D011 (TCLP Metals), D012-D043 (organic pesticides and characteristic organics), and F001, F002, F003A, and F005A (halogenated/non-halogenated spent solvents).

\section{LDR Treatment Standard}

- D004 = concentration based standard $=5.0 \mathrm{mg} / \mathrm{l}, \mathrm{TCLP}$

- D005 = concentration based standard $=21 \mathrm{mg} / \mathrm{l}, \mathrm{TCLP}$

- D006 $=$ concentration based standard $=0.11 \mathrm{mg} / \mathrm{l}, \mathrm{TCLP}$

- D007 $=$ concentration based standard $=0.60 \mathrm{mg} / \mathrm{l}, \mathrm{TCLP}$

- D008 $=$ concentration based standard $=0.75 \mathrm{mg} / \mathrm{l}, \mathrm{TCLP}$

- D009 $=$ concentration based standard $=0.025 \mathrm{mg} / \mathrm{l}$, TCLP

- $\mathrm{D} 010=$ concentration based standard $=5.7 \mathrm{mg} / \mathrm{l}, \mathrm{TCLP}$

- D011 = concentration based standard $=0.14 \mathrm{mg} / \mathrm{l}, \mathrm{TCLP}$

- $\mathrm{D} 012=$ concentration based standard $=0.13 \mathrm{mg} / \mathrm{kg}$

- D013 \& D031 = concentration based standard $=0.066 \mathrm{mg} / \mathrm{kg}$

- D014 = concentration based standard $=0.18 \mathrm{mg} / \mathrm{kg}$

- D015 = concentration based standard $=2.6 \mathrm{mg} / \mathrm{kg}$

- D016, D018, \& D032 = concentration based standard $=10.0 \mathrm{mg} / \mathrm{kg}$

- D017 $=$ concentration based standard $=7.9 \mathrm{mg} / \mathrm{kg}$ 
- D019, D021, D022, D027, D028, D029, D039, D040, \& D043 = concentration based standard = $6.0 \mathrm{mg} / \mathrm{kg}$

- $\mathrm{D} 020=$ concentration based standard $=0.26 \mathrm{mg} / \mathrm{kg}$

- D023, D024, D025, \& D033 = concentration based standard $=5.6 \mathrm{mg} / \mathrm{kg}$

- $\mathrm{D} 026=$ concentration based standard $=11.2 \mathrm{mg} / \mathrm{kg}$

- $\mathrm{D} 030=$ concentration based standard $=140 \mathrm{mg} / \mathrm{kg}$

- D034 = concentration based standard $=30 \mathrm{mg} / \mathrm{kg}$

- D035 = concentration based standard $=36 \mathrm{mg} / \mathrm{kg}$

- D036 = concentration based standard = $14 \mathrm{mg} / \mathrm{kg}$

- D037, D041, \& D042 = concentration based standard = $7.4 \mathrm{mg} / \mathrm{kg}$

- D038 = concentration based standard $=16 \mathrm{mg} / \mathrm{kg}$

- F001 \& F002= concentration based standard $=6.0-30 \mathrm{mg} / \mathrm{kg}$

- $\mathrm{F} 003=$ concentration based standard $=0.75 \mathrm{mg} / \mathrm{l} \mathrm{TCLP}-160 \mathrm{mg} / \mathrm{kg}$

- $\mathrm{F} 005$ = concentration based standards = $4.8 \mathrm{mg} / \mathrm{l}$, TCLP-170 mg/kg, except 2-Ethoxyethanol and 2-Nitropropane $=\mathrm{CMBST}$

- $\mathrm{F} 006=$ concentration based standards $=0.11-11 \mathrm{mg} / \mathrm{l} \mathrm{TCLP}$

- $\mathrm{U} 002=$ concentration based standard $=160 \mathrm{mg} / \mathrm{kg}$

- $\mathrm{U} 003=$ concentration based standard $=38 \mathrm{mg} / \mathrm{kg}$ or CMBST

- $\mathrm{U} 019=$ concentration based standard $=10 \mathrm{mg} / \mathrm{kg}$

- $\mathrm{U} 037=$ concentration based standard $=6.0 \mathrm{mg} / \mathrm{kg}$

- $\mathrm{U} 080=$ concentration based standard $=30 \mathrm{mg} / \mathrm{kg}$

- $\mathrm{U} 123=$ specified technology $=$ CMBST

- $\mathrm{U} 127=$ concentration based standard $=10 \mathrm{mg} / \mathrm{kg}$

- $\mathrm{U} 131=$ concentration based standard $=30 \mathrm{mg} / \mathrm{kg}$

- $\mathrm{U} 159=$ concentration based standard $=36 \mathrm{mg} / \mathrm{kg}$

- $\mathrm{U} 165=$ concentration based standard $=5.6 \mathrm{mg} / \mathrm{kg}$

- $\mathrm{U} 188=$ concentration based standard $=6.2 \mathrm{mg} / \mathrm{kg}$

- $\mathrm{U} 210=$ concentration based standard $=6.0 \mathrm{mg} / \mathrm{kg}$

- U211 = concentration based standard $=6.0 \mathrm{mg} / \mathrm{kg}$

- $\mathrm{U} 220=$ concentration based standard $=10.0 \mathrm{mg} / \mathrm{kg}$

- $\mathrm{U} 226=$ concentration based standard $=6.0 \mathrm{mg} / \mathrm{kg}$

- $\mathrm{U} 228=$ concentration based standard $=6.0 \mathrm{mg} / \mathrm{kg}$

- $\mathrm{U} 239=$ concentration based standard $=30 \mathrm{mg} / \mathrm{kg}$

- Alternate debris technology may be applied

- D001 through D043 nonwastewaters to be land disposed must be treated to meet the Universal Treatment Standards (UTS) for any underlying hazardous constituents (UHC) that may be present.

Since a portion of this waste stream includes wastes generated at CIF, LDR Treatment Standards are reflected in the waste fed to CIF. Specific information on treatment standards can be acquired by looking at specific wastes in Volume II.

For other constituents of waste stream SR-W003, LDR Treatment Standards are concentration-based standards ranging from $0.066 \mathrm{mg} / \mathrm{kg}$ to $170 \mathrm{mg} / \mathrm{kg}$ or with a specified technology of combustion.

\section{Waste Characterization}

- Process knowledge is used to characterize the waste stream.

- Confidence level is high based upon known composition of the solvents used in the process generating this waste.

\section{Radiological Characterization}

- Alpha emitter, $\mathrm{Pu}^{238}$

- Beta/gamma emitter, $\mathrm{Cs}^{137}$

- Waste is contact handled.

- Mixed low-level waste 


\section{SR-W012, Toxic Characteristic Solids}

This waste stream contains job control waste from previous treatment facilities such as In-Tank Precipitation (ITP), CIF, and various clean-up materials from other site generators such as plastic decon beads, rags, wipes, absorbents, mopheads, gloves, etc., contaminated with toxic characteristic waste and radioactive materials. This waste stream also contains solid chemical oxidizers generated by site laboratories and other site activities. The waste stream is a collection of similar debris whose LDR treatment standards can be met by thermal treatment. The list of waste codes indicates the components, which may be present in the waste. Waste from specific areas within SRS may not contain all the waste codes. Waste stream SR-W043, Lab Waste with Tetraphenyl Borate has been consolidated into this stream.

Volume

- Volume data for this waste stream can be found in Chapter 11.

Waste Stream Composition

- Organic debris

Waste Code

- D001 (ignitable high TOC)

- D005 (TCLP Ba)

- D007 (TCLP Cr)

- D009A (TCLP Hg)

- D011 (TCLP Ag)

- D035 (Methyl Ethyl Ketone)

- D004 (TCLP As)

- D006A (TCLP Cd)

- D008A (TCLP Pb)

- D010 (TCLP Se)

- D018 (Benzene)

- Nonwastewater

Since this waste stream includes incinerable clean-up materials from CIF, waste codes could include any of the characteristic wastes CIF was permitted to treat during start up and operation. Refer to the waste code list for SRW046 and SR-W047 for a list of all the waste codes that fed to CIF.

For that portion of waste stream SR-W012 generated from other locations at SRS, waste codes include D004 D011, D018, and D035.

\section{LDR Treatment Standard}

- D001 = specified technology $=$ RORGS or CMBST

- D004 = concentration based standard $=5.0 \mathrm{mg} / \mathrm{l} \mathrm{TCLP}$

- D005 = concentration based standard $=21 \mathrm{mg} / \mathrm{l} \mathrm{TCLP}$

- D006 $=$ concentration based standard $=0.11 \mathrm{mg} / \mathrm{TCLP}$

- D007 $=$ concentration based standard $=0.60 \mathrm{mg} / \mathrm{l}$ TCLP

- $\mathrm{D} 008=$ concentration based standard $=0.75 \mathrm{mg} / \mathrm{l}$ TCLP

- D009 $=$ concentration based standard $=0.025 \mathrm{mg} / \mathrm{l}$ TCLP

- $\mathrm{D} 010=$ concentration based standard $=5.7 \mathrm{mg} / \mathrm{l} \mathrm{TCLP}$

- $\mathrm{D} 011=$ concentration based standard $=0.14 \mathrm{mg} / 1 \mathrm{TCLP}$

- D018 = concentration based standard $=10 \mathrm{mg} / \mathrm{kg}$

- D035 = concentration based standard $=36 \mathrm{mg} / \mathrm{kg}$

- Alternate debris technology may be applied.

- D001 through D043 nonwastewaters to be land disposed must be treated to meet Universal Treatment Standards (UTS) for any underlying hazardous constituents (UHC) that may be present.

Since a portion of this waste stream includes wastes generated at CIF, LDR Treatment Standards are reflected in the characteristic wastes fed to CIF. Specific information on treatment standards can be acquired by looking at specific wastes in Volume II. For other constituents of waste stream SR-W012, LDR Treatment Standards are concentration based ranging from $0.2 \mathrm{mg} / \mathrm{L}$ to $100 \mathrm{mg} / \mathrm{L}$. 


\section{Waste Characterization}

- Process knowledge is used to characterize the waste stream.

- Confidence level is medium based on knowledge of contaminates present in the waste or from knowledge of the components in spilled material. However, sampling and analysis have not validated the characterization.

\section{Radiological Characterization}

- Alpha $\left(\mathrm{U}^{235}, \mathrm{Pu}^{238}, \mathrm{Pu}^{239}\right)$ emitters are present.

- Beta/gamma $\left(\mathrm{Cs}^{137}\right.$ and $\left.\mathrm{Sr}^{90}\right)$ emitters may be present.

- Waste is contact handled.

- Mixed low-level waste

\section{SR-W018, Listed Incinerable Solids}

This waste stream consists of F006 job control waste, remediation waste from M-Area and other site operations, process control waste from M-Area Vendor Treatment operations and remediation activities.

\section{Volume}

- Volume data for this waste stream can be found in Chapter 11.

Waste Stream Composition

- Organic debris

Waste Code

- D006 (TCLP Cd)

- D008 (TCLP Pb)

- D018 (Benzene)

- F005 (Halogenated and nonhalogenated spent solvents)

- F006 (metal plating line waste, without cyanide)

- U080 ((Methylene chloride)

- Nonwastewater

LDR Treatment Standard

- $\mathrm{D} 006=$ concentration based standard $=0.11 \mathrm{mg} / \mathrm{l}$ TCLP

- D008 $=$ concentration based standard $=0.75 \mathrm{mg} / \mathrm{l}$ TCLP

- D018 $=$ concentration based standard $=10.0 \mathrm{mg} / \mathrm{l} \mathrm{TCLP}$

- F005 = concentration based standards $=4.8 \mathrm{mg} / \mathrm{l} \mathrm{TCLP}-170 \mathrm{mg} / \mathrm{kg}$, except 2-Ethoxyethanol and 2-Nitropropane $=$ CMBST

- F006 = concentration based standards $=0.11-11 \mathrm{mg} / \mathrm{l} \mathrm{TCLP}$

- $\mathrm{U} 080=$ concentration based standard $=30 \mathrm{mg} / \mathrm{kg}$

Waste Characterization

- Process knowledge and sampling and analysis are used to characterize the waste.

- Confidence level high due to availability of sample results and knowledge the process generates listed waste.

- Primary contaminant is Ni. Others included are $\mathrm{Cd}, \mathrm{Cr}, \mathrm{Pb}$, and $\mathrm{Ag}$, but these are below RCRA LDR concentration standards.

Radiological Characterization 
- Typical activity is $5.0 \mathrm{E}-1 \mathrm{nCi} / \mathrm{g}$

- Alpha emitters are $\mathrm{U}^{234}, \mathrm{U}^{235}, \mathrm{U}^{236}$, and $\mathrm{U}^{238}$

- Waste is contact handled.

- Mixed low-level waste

\section{SR-W035, Mixed Waste Oil-Sitewide}

Waste oil is generated from sitewide preventative maintenance programs such as changing refrigeration oil in the Separations Area chillers and waste oil from lubricating and hydraulic oil change-outs from CIF equipment, also includes diesel fuel and ethylene glycol. Routinely, this is a nonradioactive used oil that could be recycled for energy recovery. However, some drums may have detectable levels of radionuclides, thus preventing recycling. Hydraulic or lubricating oil used in chillers often becomes contaminated with Freon ${ }^{\circledR}$, the refrigerant. Contaminants in the Freon ${ }^{\circledR}(\mathrm{D} 019, \mathrm{D} 039, \mathrm{D} 040)$ also have been determined to make the waste oil a mixed waste. This waste stream also includes moratorium/curtailment waste that radiological analysis has shown to be mixed waste, as well as liquids formerly in waste stream SR-W078, LDR Hazardous Waste Awaiting Radiological Screening. Less than Class A limits per 10CFR 61.55

\section{Volume}

- Volume data for this waste stream can be found in Chapter 11.

Waste Stream Composition

- Organic liquid

Waste Code

- D001A (ignitable high TOC)

- D002 (corrosive nonwastewater)

- D004 (TCLP As)

- D005 (TCLP Ba)

- D006A (TCLP Cd)

- D007 (TCLP Cr)

- D008A (TCLP Pb)

- D009A (TCLP Hg)

- D010 (TCLP Se)

- D011 (TCLP Ag)

- D018 (Benzene)

- D019 (Carbon tetrachloride)

- D022 (Chloroform)

- D029 (1,1-Dichlorehtylene)

- D039 (Tetrachloroethylene)

- D040 (Trichloroethylene)

- F001 (Spent halogenated degreasing solvents)

- F005 (Halogenated and nonhalogenated solvents)

- U123 (Formic acid)

- Nonwastewater

This waste stream is forecasted to include wastes generated by CIF operations. Those codes would depend on the specific generation episode at CIF; potentially, any of the many waste codes included in the CIF RCRA permit could apply.

LDR Treatment Standard

- D001A = specified technology = REORGS, CMBST or POLYM 
- D002 $=$ specified technology $=$ DEACT

- D004 = concentration based standard $=5.0 \mathrm{mg} / \mathrm{l} \mathrm{TCLP}$

- D005 = concentration based standard $=21 \mathrm{mg} / \mathrm{l} \mathrm{TCLP}$

- $\mathrm{D} 006=$ concentration based standard $=0.11 \mathrm{mg} / \mathrm{l}$ TCLP

- $\mathrm{D} 007=$ concentration based standard $=0.60 \mathrm{mg} / \mathrm{l}$ TCLP

- $\mathrm{D} 008=$ concentration based standard $=0.75 \mathrm{mg} / \mathrm{l}$ TCLP

- $\mathrm{D} 009=$ concentration based standard $=0.025 \mathrm{mg} / \mathrm{l}$ TCLP

- $\mathrm{D} 010=$ concentration based standard $=5.7 \mathrm{mg} / \mathrm{l} \mathrm{TCLP}$

- $\mathrm{D} 011=$ concentration based standard $=0.14 \mathrm{mg} / \mathrm{l}$ TCLP

- $\mathrm{D} 018=$ concentration based standard $=10.0 \mathrm{mg} / \mathrm{kg}$

- D019, D022, D029, D039, D040 = concentration based standard $=6.0 \mathrm{mg} / \mathrm{kg}$

- F001 = concentration based standard $=6-30 \mathrm{mg} / \mathrm{kg}$ TCLP

- $\mathrm{F} 005=$ concentration based standards $=4.8 \mathrm{mg} / \mathrm{l} \mathrm{TCLP}-170 \mathrm{mg} / \mathrm{kg}$; except 2-Ethoxyethanol, 2-Nitropropane = CMBST

- $\mathrm{U} 123=$ specified technology $=$ CMBST

- D001 through D043 nonwastewaters to be land disposed must be treated to meet the Universal Treatment Standards (UTS) for any underlying hazardous constituents (UHC) that may be present.

\section{Waste Characterization}

- Sampling and analysis are used to characterize the waste stream.

- Confidence level is high because of TCLP results.

- TCLP has been run on nonradioactive Freon ${ }^{\circledR} 11$ only. Radiological Characterization

- Tritium is present in waste stream.

- Waste is contact handled.

- Mixed low-level waste.

\section{SR-W042, Paints and Thinners}

This waste stream consists of radioactively contaminated, off-specification waste paint, spent paint solvents, and paint chips from paint removal activities.

Volume

- Volume data for this waste stream can be found in Chapter 11.

Waste Stream Composition

- Organic sludge/particulate

Waste Code

- D001C (ignitable low TOC nonwastewaters)

- D004 (TCLP As)

- D005 (TCLP Ba)

- D006A (TCLP Cd)

- D007 (TCLP Cr)

- D008A (TCLP Pb)

- D009A (TCLP Hg)

- D011 (TCLP Ag)

- D018 (Benzene)

- D035 (Methyl Ethyl Ketone)

- D038 (Pyridine)

- F003A (Xylene, Acetone)

- F005A (Halogenated and nonhalogenated spent solvents)

- D010 (TCLP Se)

LDR Treatment Standard

- D001 $=$ specified technology $=$ REORG or CMBST 
- $\mathrm{D} 004=$ concentration based standard $=5.0 \mathrm{mg} / \mathrm{l} \mathrm{TCLP}$

- D005 = concentration based standard $=21 \mathrm{mg} / \mathrm{l}, \mathrm{TCLP}$

- D006 $=$ concentration based standard $=0.11 \mathrm{mg} / \mathrm{l}, \mathrm{TCLP}$

- $\mathrm{D} 007=$ concentration based standard $=0.60 \mathrm{mg} / \mathrm{l}$, TCLP

- D008 $=$ concentration based standard $=0.75 \mathrm{mg} / \mathrm{l}, \mathrm{TCLP}$

- D009 $=$ concentration based standard $=0.025 \mathrm{mg} / \mathrm{l}$, TCLP

- $\mathrm{D} 010=$ concentration based standard $=5.7 \mathrm{mg} / \mathrm{l} \mathrm{TCLP}$

- D011 = concentration based standard $=0.14 \mathrm{mg} / \mathrm{l}$, TCLP

- D018 $=$ concentration based standard $=10 \mathrm{mg} / \mathrm{kg}$, UTS $=10 \mathrm{mg} / \mathrm{kg}$

- D035 $=$ concentration based standard $=36 \mathrm{mg} / \mathrm{kg}, \mathrm{UTS}=36 \mathrm{mg} / \mathrm{kg}$

- D038 $=$ concentration based standard $=16 \mathrm{mg} / \mathrm{kg}$, UTS $=16 \mathrm{mg} / \mathrm{kg}$

- $\mathrm{F} 003=$ concentration based standards $=0.75 \mathrm{mg} / \mathrm{l}, \mathrm{TCLP}-160 \mathrm{mg} / \mathrm{kg}$

- $\mathrm{F} 005=$ concentration based standards $=4.8 \mathrm{mg} / \mathrm{l}$, TCLP $-170 \mathrm{mg} / \mathrm{kg}$, except for $2-$ Ethozyethanol, and 2 Nitropropane $=$ CMBST

- D001 through D043 nonwastewaters to be land disposed must be treated to meet Universal Treatment Standards (UTS) for any underlying hazardous constituent (UHC) that may be present.

- Future generation may include listed spent solvent codes F001, F002, F003, and /or F005.

Waste Characterization

- Confidence level is high because sample and analysis available.

Radiological Characterization

- Alpha and beta/gamma emitters and tritium

- Waste is contact handled

- Mixed low-level waste

\section{SR-W051, Spent Filter Cartridges and Carbon Filter Media}

The waste stream consists of incinerable filters and filter media. Examples of this waste stream include filters in Naval Fuels used to remove particles contaminated with mercury salts and depleted uranium from the process flow stream. Also included in this waste are CIF feed tank and offgas HEPA filters.

Volume

- Volume data for this waste stream can be found in Chapter 11.

Waste Stream Composition

- Heterogeneous debris

Waste Code

- D004 (TCLP As)

- D005 (TCLP Ba)

- D006 (TCLP Cd)

- D007 (TCLP Cr)

- D018 (Benzene)

- D021 (Chlorobenzene)

- D035 (Methyl Ethyl Ketone)

- D040 (Trichloroethylene)
- D009A (low TCLP Hg)

- D010 (TCLP Se)

- D011 (TCLP Ag)

- D008A (TCLP Pb)

- D019 (Carbon tetrachloride)

- D034 (Hexachloroethane)

- D039 (Tetrachloroethylene)

- Nonwastewater 
Waste from CIF could contain all the listed waste codes that are fed to CIF and any characteristic waste codes determined by analysis. Refer to waste codes listed for SR-W046 and SR-W047 for a complete listing of all waste codes permitted.

\section{LDR Treatment Standard}

- D004 = concentration based standard $=5.0 \mathrm{mg} / 1 \mathrm{TCLP}$

- D005 $=$ concentration based standard $=21 \mathrm{mg} / \mathrm{l} \mathrm{TCLP}$

- $\mathrm{D} 006=$ concentration based standard $=0.11 \mathrm{mg} / \mathrm{l}$ TCLP

- $\mathrm{D} 007=$ concentration based standard $=0.60 \mathrm{mg} / \mathrm{l}$ TCLP

- D008 $=$ concentration based standard $=0.75 \mathrm{mg} / \mathrm{l} \mathrm{TCLP}$

- $\mathrm{D} 009=$ concentration based standard $=0.025 \mathrm{mg} / \mathrm{l}$ TCLP

- $\mathrm{D} 010=$ concentration based standard $=5.7 \mathrm{mg} / \mathrm{l} \mathrm{TCLP}$

- $\mathrm{D} 011=$ concentration based standard $=0.14 \mathrm{mg} / \mathrm{l}$ TCLP

- D018 $=$ concentration based standard $=10 \mathrm{mg} / \mathrm{kg}$

- $\mathrm{D} 019=$ concentration based standard $=6.0 \mathrm{mg} / \mathrm{kg}$

- $\mathrm{D} 021=$ concentration based standard $=6.0 \mathrm{mg} / \mathrm{kg}$

- $\mathrm{D} 022=$ concentration based standard $=6.0 \mathrm{mg} / \mathrm{kg}$

- D032 = concentration based standard $=10 \mathrm{mg} / \mathrm{kg}$

- D034 = concentration based standard $=30 \mathrm{mg} / \mathrm{kg}$

- D039 $=$ concentration based standard $=6.0 \mathrm{mg} / \mathrm{kg}$

- $\mathrm{D} 040=$ concentration based standard $=6.0 \mathrm{mg} / \mathrm{kg}$

- Alternative debris technology may be applied.

- D001 through D043 nonwastewaters to be land disposed must be treated to meet Universal Treatment Standards (UTS) for any underlying hazardous constituents (UHC) that may be present.

CIF waste will have treatment standards that are reflected in the waste fed to CIF and any applicable characteristic waste. Specific information on treatment standards can be acquired by looking at specific wastes in Volume II.

\section{Waste Characterization}

- Process knowledge and sample analysis are used to characterize the waste stream.

- Confidence level is high-based sample analysis.

\section{Radiological Characterization}

- Beta/gamma emitters are present.

- Alpha emitters $\left(\mathrm{U}^{233}, \mathrm{U}^{234}\right.$, and $\left.\mathrm{U}^{238}\right)$ are present.

- Waste is contact handled.

- Mixed low-level waste

\section{SR-W055, Job Control Waste Containing Solvent Contaminated Wipes}

This waste is sitewide operations generated job waste, including radiologically contaminated plastic huts, protective clothing, contaminated metal tools, glass, paper, and cardboard that is suspected to have been mixed with solvent contaminated wipes. Job waste has been declared mixed waste according to the Mixture Rule. Wastes in this stream were declared mixed waste January 25, 1990. SRS has modified procedures and practices regarding solvent contaminated wipes generation and management to eliminate or substantially reduce this type of waste.

Volume

- Volume data for this waste stream can be found in Chapter 11.

Waste Stream Composition

- Organic debris 
Waste Code

- F001 (Spent halogenated degreasing solvents)

- F002 (Spent halogenated solvents)

- F003A (Spent nonhalogenated solvents)

- F005A (Halogenated and nonhalogenated spent solvents)

- Nonwastewater

LDR Treatment Standard

- F001 \& F002 = concentration based standards $=6.0-30 \mathrm{mg} / \mathrm{kg}$

- $\mathrm{F} 003=$ concentration based standards $=0.75 \mathrm{mg} / \mathrm{l} \mathrm{TCLP}-160 \mathrm{mg} / \mathrm{kg}$

- F005 = concentration based standards $=4.8 \mathrm{mg} / \mathrm{l}$ TCLP-170 mg/kg, except for 2-Ethoxyethanol, and 2Nitropropane $=$ CMBST

- Alternate debris technology may be applied.

\section{Waste Characterization}

- Process knowledge is used to characterize the waste stream.

- Confidence level is medium based on the use of process knowledge to characterize waste. Also, other waste in the waste stream may not actually be contaminated with solvents but are characterized as such, according to the Mixture Rule.

\section{Radiological Characterization}

- Beta/gamma emitters are present.

- Waste is contact handled.

- Mixed low-level waste.

\section{SR-W070, Mixed Waste from Laboratory Samples}

This waste stream consists of incinerable lab waste from the analytical testing of groundwater samples taken from the site and processed at onsite or commercial, offsite laboratories. It includes sample residues, unused samples, and related laboratory wastes. In addition, this waste stream may include used and unused laboratory reagents. Wastes included in this stream may consist of both liquid and solid (e.g. soil) matrices. Radiological levels and hazardous constituent levels will depend upon the source location.

Volume

- Volume data for this waste stream can be found in Chapter 11.

Waste Stream Composition

- Liquids

- Solids

Waste Code

- D001C (ignitable, low TOC)

- D002 (corrosive, nonwastewater)

- D004 (TCLP As)

- D005 (TCLP Ba) 
- D006A (TCLP Cd)

- D007 (TCLP 1,1-Dichlorethylene)

- D008A (TCLP Pb)

- D009A (TCLP Hg)

- D010 (TCLP Se)

- D011 (TCLP Ag)

- D018 (TCLP Benzene)

- D019 (Carbon tetrachloride)

- D035 (Methyl Ethyl Ketone)

- D036 (Nitrobenzene)

- D038 (Pyridine)

- D039 (Tetrachloroethylene)

- D040 (Trichloroethylene)

- F001 (unspecified)

- F002 (Methylene chloride)

- F003A (Ethyl ether)

- F004X (unspecified solvents)

- F005 (spent non-hologenated solvents)

- F006 (metal plating waste without cyanide)

- P098 (Potassium cyanide)

- P120 (Vanadium pentoxide)

- U002 (Acetone)

- U003 (Acetonitrile)

- U019 (Benzene)

- U037 (Chlorobenzene)

- U045 (Chloromethane)

- U080 (Methylene chloride)

- U123 (Formic acid)

- U127 (Hexachlorobenzene)

- U131 (Hexachloroethane)

- U144 (Lead acetate)

- U159 (Methyl Ethyl Ketone)

- U188 (Phenol)

- U165 (Naphthalene)

- U204 (Selenium dioxide)

- U210 (Tetrachloroethylene)

- U211 (Carbon tetrachloride)

- U220 (Toluene)

- U226 (1,1,1-Trichloroethane)

- U228 (Trichloroethyene)

- U239 (Xylenes)

- Nonwastewater

LDR Treatment Standard

- D001 $=$ specified technology $=$ DEACT, or RORGS or CMBST

- D002 $=$ specified technology $=$ DEACT

- D004 = concentration based standard $=5.0 \mathrm{mg} / \mathrm{l}$, TCLP

- D005 $=$ concentration based standard $=21 \mathrm{mg} / \mathrm{l}$, TCLP 
- D006 $=$ concentration based standard $=0.11 \mathrm{mg} / \mathrm{l}$, TCLP

- $\mathrm{D} 007=$ concentration based standard $=0.60 \mathrm{mg} / \mathrm{l}$, TCLP

- D008 $=$ concentration based standard $=0.75 \mathrm{mg} / \mathrm{l}, \mathrm{TCLP}$

- D009 = concentration based standard $=0.025 \mathrm{mg} / \mathrm{l}$, TCLP

- $\mathrm{D} 010=$ concentration based standard $=5.7 \mathrm{mg} / \mathrm{l}, \mathrm{TCLP}$

- D018 $=$ concentration based standard $=10 \mathrm{mg} / \mathrm{kg}$

- D035 = concentration based standard $=36 \mathrm{mg} / \mathrm{l} \mathrm{TCLP}$

- D036 = concentration based standard $=14 \mathrm{mg} / \mathrm{l} \mathrm{TCLP}$

- D019 $=$ concentration based standard $=6.0 \mathrm{mg} / \mathrm{kg}$

- $\mathrm{D} 038=$ concentration based standard $=16 \mathrm{mg} / \mathrm{kg}$

- $\mathrm{D} 039=$ concentration based standard $=6.0 \mathrm{mg} / \mathrm{kg}$

- D040 $=$ concentration based standard $=6.0 \mathrm{mg} / \mathrm{kg}$

- $\mathrm{F} 001=$ concentration based standard $=6-30 \mathrm{mg} / \mathrm{kg}$

- $\mathrm{F} 002=$ concentration based standard $=30 \mathrm{mg} / \mathrm{kg}$

- $\mathrm{F} 003=$ concentration based standard $=160 \mathrm{mg} / \mathrm{kg}$

- $\mathrm{F} 004=$ concentration based standard $=6-160 \mathrm{mk} / \mathrm{kg}$

- F005 = concentration based standard $=4.8 \mathrm{mg} / \mathrm{L}$, TCLP through $170 \mathrm{mg} / \mathrm{kg}$; except $2-$ Ethoxyethanol, 2 Nitropropane $=$ CMBST

- F006 $=$ concentration based standard $=0.19-5.0 \mathrm{mg} / \mathrm{l}$, TCLP

- $\mathrm{P} 098=$ concentration based standard $=30-590 \mathrm{mg} / \mathrm{kg}$ TCLP

- $\mathrm{P} 120=$ specified technology $=\mathrm{STABL}$

- $\mathrm{U} 002=$ concentration based standard $=160 \mathrm{mg} / \mathrm{kg}$

- $\mathrm{U} 003=$ specified technology $=$ CMBST

- $\mathrm{U} 019=$ concentration based standard $=10 \mathrm{mg} / \mathrm{kg}$

- $\mathrm{U} 037=$ concentration based standard $=6 \mathrm{mg} / \mathrm{kg}$

- $\mathrm{U} 045=$ concentration based standard $=30 \mathrm{mg} / \mathrm{kg}$

- $\mathrm{U} 080=$ concentration based standard $=30 \mathrm{mg} / \mathrm{kg}$

- $\mathrm{U} 123=$ specified technology $=$ CMBST

- $\mathrm{U} 127=$ concentration based standard $=10 \mathrm{mg} / \mathrm{kg}$

- $\mathrm{U} 131=$ concentration based standard $=30 \mathrm{mg} / \mathrm{kg}$

- $\mathrm{U} 144=$ concentration based standard $=0.75 \mathrm{mg} / \mathrm{L}$

- $\mathrm{U} 159=$ concentration based standard $=36 \mathrm{mg} / \mathrm{kg}$

- $\mathrm{U} 165=$ concentration based standard $=5.6 \mathrm{mg} / \mathrm{kg}$

- $\mathrm{U} 188=$ concentration based standard $=6.2 \mathrm{mg} / \mathrm{kg}$

- $\mathrm{U} 204=$ concentration based standard $=5.7 \mathrm{mg} / \mathrm{L}$ TCLP

- $\mathrm{U} 210=$ concentration based standard $=6 \mathrm{mg} / \mathrm{kg}$

- $\mathrm{U} 211=$ concentration based standard $=6 \mathrm{mg} / \mathrm{kg}$

- $\mathrm{U} 220=$ concentration based standard $=10 \mathrm{mg} / \mathrm{kg}$

- $\mathrm{U} 226=$ concentration based standard $=6 \mathrm{mg} / \mathrm{kg}$

- $\mathrm{U} 228=$ concentration based standard $=6.0 \mathrm{mg} / \mathrm{kg}$

- $\mathrm{U} 239=$ concentration based standard $=30 \mathrm{mg} / \mathrm{kg}$

- D001 through D043 nonwastewaters to be land disposed must be treated to meet Universal Treatment Standards (UTS) for any underlying hazardous constituents (UHC) that may be present.

\section{Waste Characterization}

- Sampling and analysis are used to characterize the waste stream.

- Confidence level is high because waste has been characterized by sampling and analysis.

Radiological Characterization

- $\mathrm{H}^{3}, \mathrm{Am}^{241}, \mathrm{Cs}^{137}, \mathrm{Pu}^{238}, \mathrm{Pu}^{239}, \mathrm{Sr}^{90}, \mathrm{U}^{234}, \mathrm{U}^{235}, \mathrm{U}^{236}$, and $\mathrm{U}^{234}$

- $<100 \mathrm{nCi} / \mathrm{g}$

- Contact handled 


\section{SR-W071, Wastewater Suitable for Thermal Treatment or Stabilization}

This waste stream consists of wastewaters that are suitable for thermal treatment, stabilization, or macroencapsulation. These wastewaters include aqueous wastes with listed organic constituents that can be incinerated, stabilized, or macroencapsulated in compliance with LDR requirements. Examples include wastewater collected from CIF sumps and aqueous solutions with organic contaminants such as a small volume of outdated ethyl ether in water stored in an analytical laboratory satellite accumulation area. It is anticipated that other wastes of a similar nature could be generated in the future. Wastewaters that do not meet the requirements of 268.3 (c) will be stabilized.

\section{Volume}

- Volume data for this waste stream can be found in Chapter 11.

Waste Stream Composition

- Aqueous liquid

Waste Code

- D002 (corrosive, wastewater)

- D011 (TCLP Ag)

- D004 (TCLP As)

- D005 (TCLP Ba)

- D006A (TCLP Cd)

- D007 (TCLP Cr)

- D008A (TCLP Pb)

- D009A (TCLP Hg)

- F001 (Spent halogenated degreasing solvents)

- F002 (Spent halogenated solvents)

- F003A (Spent nonhalogenated solvents)

- F005A (Halogenated and nonhalogenated solvents)

- D010 (TCLP Se)

- Nonwastewater

- Wastewater

\section{LDR Treatment Standard}

- D002 $=$ specified technology $=$ DEACT and meet UTS

- D004 = concentration based standard $=5.0 \mathrm{mg} / \mathrm{l}$, TCLP

- D005 = concentration based standard $=21 \mathrm{mg} / \mathrm{l}$, TCLP

- $\mathrm{D} 006=$ concentration based standard $=0.11 \mathrm{mg} / \mathrm{l}, \mathrm{TCLP}$

- $\mathrm{D} 007=$ concentration based standard $=0.60 \mathrm{mg} / \mathrm{l}$, TCLP

- D008 = concentration based standard $=0.75 \mathrm{mg} / \mathrm{l}, \mathrm{TCLP}$

- D009 $=$ concentration based standard $=0.025 \mathrm{mg} / \mathrm{l}$, TCLP

- $\mathrm{D} 010=$ concentration based standard $=5.7 \mathrm{mg} / \mathrm{l}, \mathrm{TCLP}$

- $\mathrm{D} 011=$ concentration based standard $=0.14 \mathrm{mg} / \mathrm{l}$, TCLP

- $\mathrm{F} 001=$ concentration based standard $=6-30 \mathrm{mg} / \mathrm{kg}$

- $\mathrm{F} 002=$ concentration based standard $=6-30 \mathrm{mg} / \mathrm{kg}$

- $\mathrm{F} 003=$ concentration based standard $=0.75 \mathrm{mg} / \mathrm{l}$, TCLP through $60 \mathrm{mg} / \mathrm{kg}$

- F005 = concentration based standard $=4.8 \mathrm{mg} / \mathrm{l}$, TCLP through $170 \mathrm{mg} / \mathrm{kg}$; except 2-Ethoxyethanol, 2Nitropropane $=$ CMBST

- D001 through D043 nonwastewaters to be land disposed must be treated to meet Universal Treatment Standards (UTS) for any underlying hazardous constituent (UHC) that may be present.

Waste from CIF could contain any or all of the listed waste codes that are fed to CIF. The CIF RCRA Part B permit should be consulted for the complete list.

\section{Waste Characterization}

- Radiological and chemical analysis will be performed on wastes that are included in this waste stream. 
- Confidence level is high because sampling and analysis has been performed.

\section{Radiological Characterization}

- Contact handled

- Mixed low-level waste

- Radiological characterization of future waste streams cannot be determined at this time. Both alpha and beta/gamma emitters are expected.

\section{SR-W080, Charleston Naval Shipyard Waste (CN-W001, CN-W004)}

This waste stream is composed of flammable or incinerable solids and debris containing potassium chromate and/or contaminated with chromium and/or lead generated from ship overhaul, decommissioning, and routine shipyard maintenance. Charleston Naval Shipyard waste was shipped to SRS and placed in RCRA permitted storage, as concurred by SCDHEC, in December 1995 prior to the closure of the Charleston Naval Shipyard in April 1996. The Approved Site Treatment Plan had three waste streams listed for the CNS waste. However, upon shipment, only two waste streams were received at SRS for storage. The third waste stream, Flammable Organic Debris (CN-W007), was never generated by the Naval Yard.

Volume

- Volume data for this waste stream can be found in Chapter 11.

Waste Codes

- D007 (TCLP Cr)

- D008A (TCLP Pb)

- Nonwastewater

LDR Treatment Standard

- $\mathrm{D} 007=$ Concentration Based Standard $=0.60 \mathrm{mg} / 1 \mathrm{TCLP}$

- D008 $=$ Concentration Based Standard $=0.75 \mathrm{mg} / 1$ TCLP

- D001 through D043 nonwastewaters to be land disposed must be treated to meet Universal Treatment Standards (UTS) for any underlying hazardous constituents (UHC) that may be present.

Waste Characterization

- Process knowledge

Radiological Characterization

- Beta/gamma emitters are present.

- Primary radionuclide constituent $=\mathrm{Co}^{60}$

- Contact handled

\section{SR-W081, Reactive/Ignitable Waste}

This waste stream is currently composed of SRNL laboratory wastes generated as a result of preparing metal specimens for examination in the transmission electron microscope. Metal disks were electropolished using a solution of perchloric acid, butycellosolve, and methanol. Because of its reactivity, the spent perchloric acid solution is deactivated with distilled water in the storage area.

Volume

- Volume data for this waste stream can be found in Chapter 11. 
Waste Stream Composition

- Aqueous liquid

Waste Codes

- D001A (ignitable high TOC)

- D003E (other reactive)

LDR Treatment Standard

- D001 $=$ specified technology $=$ RORGS or CMBST

- D003 $=$ specified technology $=$ DEACT

- D001 through D043 nonwastewaters to be land disposed must be treated to meet Universal Treatment Standards (UTS) for any underlying hazardous constituent (UHC) that may be present.

Waste Characterization

- Process knowledge

- Confidence level is high based upon the known composition and quantity of chemicals used to formulate the perchloric acid polishing solution.

\section{Radiological Characterization}

- Radionuclide constituent - $\mathrm{Co}^{60}, \mathrm{H}^{3}, \mathrm{Pu}^{239}, \mathrm{Pu}^{238}$

- Contact handled

\section{SR-W087, Bettis Atomic Power Laboratory Contaminated Oil, (BT-W035 part of BT-W003)}

Volume

- Volume data for this waste stream can be found in Chapter 11.

Waste Stream Composition

- Organic debris

Waste Code

- D006 (TCLP Cd)

- D008A (TCLP Pb)

- Nonwastewater

LDR Treatment Standard

- $\mathrm{D} 006=$ concentration based standard $=0.11 \mathrm{mg} / \mathrm{L}$ TCLP

- $\mathrm{D} 008 \mathrm{~A}=$ concentration based standard $=0.75 \mathrm{mg} / \mathrm{L}$ TCLP

Waste Characterization

- Sample results

- Process knowledge

Radiological Characterization

- Beta/gamma emitters are present.

- Primary radionuclide constituent $=\mathrm{U} 233$ 
- Contact handled

\subsubsection{Commercial Vendor Deactivation Treatment}

\section{SR-W034, Calcium Metal}

Volume

- Volume data for this waste stream can be found in Chapter 11.

Waste Stream Composition

- Inorganic debris

Waste Code

- D003 (Reactivity)

LDR Treatment Standard

- $\mathrm{D} 003=$ deactivation

Waste Characterization

- Process knowledge

Radiological Characterization

- Contact handled

\subsubsection{Preferred Treatment to be Determined}

\section{SR-W091, Contaminated Debris (High Rad)}

This new stream was declared August 2004, and a treatment strategy will be submitted to SCDHEC within 1 year of declaration. A description is found below:

This waste stream consists of greater than Class A debris (metal, floor tiles, fluorescent light bulbs, instruments, and other equipment including debris generated from operations at CIF and machinery used in the remediation of various contamination sites that could not be decontaminated) contaminated with TCLP metals and radionuclides. (NOTE: this is a different stream from SR-W015, Mercury/Tritium Contaminated Equipment). This waste requires a permitted TSD for treatment since it has been in permitted storage.

Also included in this waste stream are tools and other non-incinerable items found in waste stream SR-W055, Job Control Waste Containing Solvent Contaminated Wipes and PPE, SR-W025, Solvent/TRU Job Control Waste < $100 \mathrm{nCi} / \mathrm{g}$; SR-W042, Paints and Thinners; and other waste streams shredded in preparation for treatment by combustion.

In addition to the CIF debris, this waste stream also consists of filters, job control waste from the segregation and decontamination of lead in Building 105-C, and soil associated with other equipment or debris from the M-Area 
Vendor Treatment Facility, the tank farms, and other site locations. Radioactive Hg/Ni-Cad batteries have recently been approved by EPA for macroencapsulation and are therefore included in this wastestream.

Volume

- Volume data on this waste stream can be found in Chapter 11.

Waste Stream Composition

- Inorganic debris

Waste Code 
- D004 (TCLP As)

- D005 (TCLP Ba)

- D006A (TCLP Cd)

- D007 (TCLP Cr)

- D008A (TCLP Pb)

- D009A (TCLP Hg)

- D010 (TCLP Se)

- D011 (TCLP Ag)

- D035 (Methyl ethyl ketone)

- D039 (Tetrachloroethylene)

- F001 (Unspecified solvents)

- F002 (Unspecified solvents)

- F003X (Unspecified solvents)

- F004X (Unspecified solvents)

- F005X (Unspecified solvents)

- F006 (Metal plating waste without cyanide)

- P048 (2,4-Dinitrophenol)

- P113 (Thallic Oxide)

- U002 (Acetone)

- U003 (Acetonitrile)

- U019 (Benzene)

- U037 (Chlorobenzene)

- U080 (Methylene Chloride)

- U123 (Formic Acid)

- U127 (Hexachlorobenzene)

- U131 (Hexachloroethane)

- U151 (TCLP Hg)

- U159 (Methyl Ethyl Ketone)

- U165 (Naphthalene)

- U188 (Phenol)

- U209 (1,1,2,2-Tetrachloroethane)

- U210 (Tetrachloroethylene)

- U211 (Carbon Tetrachloride)

- U220 (Toluene)

- U226 (1,1,1-Trichloroethane)

- U228 (Trichloroethylene)

- U239 (Xylenes)

- Nonwastewater 
Specific waste codes will vary depending upon where the waste was generated. Wastes from CIF contain all of the waste codes that are fed to CIF. The F-listed solvents listed reflect the inclusion of non-incinerable items found in waste streams SR-W025 and SR-W055. Items from SR-W025 and SR-W055 may contain other P- and U-listed codes.

\section{LDR Treatment Standard}

- D004 $=$ concentration based standard $=5.0 \mathrm{mg} / \mathrm{l}, \mathrm{TCLP}$

- D005 = concentration based standard $=21 \mathrm{mg} / \mathrm{l}, \mathrm{TCLP}$

- $\mathrm{D} 006=$ concentration based standard $=0.11 \mathrm{mg} / \mathrm{l}, \mathrm{TCLP}$

- D007 $=$ concentration based standard $=0.60 \mathrm{mg} / \mathrm{l}$, TCLP

- $\mathrm{D} 008=$ concentration based standard $=0.75 \mathrm{mg} / \mathrm{l}$, TCLP

- D009 $=$ concentration based standard $=0.025 \mathrm{mg} / \mathrm{l}$, TCLP

- D010 = concentration based standard $=5.7 \mathrm{mg} / \mathrm{l}, \mathrm{TCLP}$

- $\mathrm{D} 011=$ concentration based standard $=0.14 \mathrm{mg} / \mathrm{l}, \mathrm{TCLP}$

- D035 = concentration based standard $=36 \mathrm{mg} / \mathrm{kg}$

- $\mathrm{D} 039=$ concentration based standard $=0.14 \mathrm{mg} / \mathrm{l}, \mathrm{TCLP}$

- $\mathrm{F} 001=$ concentration based standard $=6-30 \mathrm{mg} / \mathrm{kg}$

- $\mathrm{F} 002=$ concentration based standard $=6-30 \mathrm{mg} / \mathrm{kg}$

- $\mathrm{F} 003=$ concentration based standard $=2.6-60 \mathrm{mg} / \mathrm{kg}$

- F004 = concentration based standard $=6-160 \mathrm{mg} / \mathrm{kg}$

- F005 $=$ concentration based standard $=10-170 \mathrm{mg} / \mathrm{kg}$

- F006 $=$ concentration based standard $=0.19-5.0 \mathrm{mg} / \mathrm{l}$, TCLP

- $\mathrm{P} 048=$ concentration based standard $=160 \mathrm{mg} / \mathrm{kg}$

- P113 = specified technology = RTHRM; or STABL

- $\mathrm{U} 002=$ concentration based standard $=160 \mathrm{mg} / \mathrm{kg}$

- $\mathrm{U} 003=$ specified technology $=$ CMBST

- $\mathrm{U} 019=$ concentration based standard $=10 \mathrm{mg} / \mathrm{kg}$

- $\mathrm{U} 037=$ concentration based standard $=6 \mathrm{mg} / \mathrm{kg}$

- $\mathrm{U} 080=$ concentration based standard $=30 \mathrm{mg} / \mathrm{kg}$

- $\mathrm{U} 123=$ specified technology $=$ CMBST

- $\mathrm{U} 127=$ concentration based standard $=10 \mathrm{mg} / \mathrm{kg}$

- $\mathrm{U} 131=$ concentration based standard $=30 \mathrm{mg} / \mathrm{kg}$

- $\mathrm{U} 151=$ concentration based standard $=0.025 \mathrm{mg} / \mathrm{l}$, TCLP

- $\mathrm{U} 159=$ concentration based standard $=36 \mathrm{mg} / \mathrm{kg}$

- $\mathrm{U} 165=$ concentration based standard $=5.6 \mathrm{mg} / \mathrm{kg}$

- $\mathrm{U} 188=$ concentration based standard $=6.2 \mathrm{mg} / \mathrm{kg}$

- $\mathrm{U} 209=$ concentration based standard $=6.0 \mathrm{mg} / \mathrm{kg}$

- $\mathrm{U} 210=$ concentration based standard $=6 \mathrm{mg} / \mathrm{kg}$

- $\mathrm{U} 211=$ concentration based standard $=6 \mathrm{mg} / \mathrm{kg}$

- $\mathrm{U} 220=$ concentration based standard $=10 \mathrm{mg} / \mathrm{kg}$

- $\mathrm{U} 226=$ concentration based standard $=6 \mathrm{mg} / \mathrm{kg}$

- $\mathrm{U} 228=$ concentration based standard $=6.0 \mathrm{mg} / \mathrm{kg}$

- $\mathrm{U} 239=$ concentration based standard $=30 \mathrm{mg} / \mathrm{kg}$

- D001 through D043 nonwastewaters to be land disposed must be treated to meet Universal Treatment Standards (UTS) for any underlying hazardous constituents (UHC) that may be present.

Waste debris from CIF will have treatment standards that are reflected in the waste fed to CIF. Specific information on treatment standards can be acquired by looking at specific wastes in Volume II.

\section{Waste Characterization}

- Process knowledge is used to characterize the waste stream.

- Confidence level is high based on knowing process history of the waste. 
Radiological Characterization

- Radioactivity will vary depending on the generation source and location.

- Waste is contact handled.

- Mixed low-level waste

\subsection{Mixed Low-Level Waste Streams Requiring Technology Development}

Due to the complexity of some waste streams, technologies have not been developed that can adequately treat the waste. Therefore treatment strategies for some waste streams have not been selected. This section addresses those mixed low-level waste streams that require technology development for the treatment of the waste stream.

\subsubsection{Development of Mobile Unit Technology}

Currently, there are no waste streams in this category.

\subsubsection{Development of Characterization Technology}

The waste stream, SR-W056, Job Control Waste with Enriched Uranium and Solvent Contaminated Wipes, previously included in this section has been determined to be nonhazardous. Currently, there are no additional waste streams requiring development of chemical characterization technology.

\subsubsection{Development of Treatment Technology}

The waste stream SR-W045, PUREX Waste (organic fraction) was previously planned to be treated by thermal treatment at the Consolidated Incineration Facility (CIF). The preferred option for this waste stream is now to develop a direct stabilization process using new technology which will allow the treatment of this waste stream to be completed on a greatly accelerated timescale and for a considerable cost-savings over the use of CIF. The selected treatment reagents added to stabilize W045 will produce physical and chemical changes to immobilize the hazardous concerns in the waste stream. The amount of reagents added will be part of the normal process of treating the waste to effectively immobilize the hazardous constituents. In the preliminary development work performed as part of the PUREX alternative treatment study, several commercial reagents were tested using waste concentrations of 50 weight percent and higher in the final wasteforms. The leachability of hazardous constituents in the final wasteforms was measured using the TCLP leach test. The hazardous constituent concentrations in the leachates were less than the LDR treatment standards, and also lower than the total concentrations in the final wasteforms. Since the leachate concentrations were lower than the wasteform concentrations, the stabilization reagents are immobilizing the hazardous constituents and reducing their mobility in the environment.

Further development is continuing to develop the specific treatment reagents and stabilization process. SR-W045, PUREX Waste

An organic solvent generated in the Plutonium/Uranium Extraction Process (PUREX) used in the Separations areas. SR-W044, Tri-Butyl-Phosphate and n-Paraffin TRU, have been combined with this waste stream.

\section{Volume}

- Volume data for this waste stream can be found in Chapter 11.

Waste Stream Composition

- Organic liquid

Waste Code 
- D004 (TCLP As)

- D005 (TCLP Ba)

- D006A (TCLP Cd)

- D007 (TCLP Cr)

- D008A (TCLP Pb)

- D009A (TCLP Hg)

- D010 (TCLP Se)

- D011 (TCLP Ag)

- D018 (Benzene)

- D040 (Trichloroethylene)

- Nonwastewater

\section{LDR Treatment Standard}

- D004 $=$ concentration based standard $=5.0 \mathrm{mg} / 1 \mathrm{TCLP}$

- D005 $=$ concentration based standard $=21 \mathrm{mg} / \mathrm{l} \mathrm{TCLP}$

- $\mathrm{D} 006=$ concentration based standard $=0.11 \mathrm{mg} / \mathrm{l} \mathrm{TCLP}$

- $\mathrm{D} 007=$ concentration based standard $=0.60 \mathrm{mg} / \mathrm{l}$ TCLP

- $\mathrm{D} 008=$ concentration based standard $=0.75 \mathrm{mg} / \mathrm{l}$ TCLP

- $\mathrm{D} 009=$ concentration based standard $=0.025 \mathrm{mg} / \mathrm{l} \mathrm{TCLP}$

- $\mathrm{D} 010=$ concentration based standard $=5.7 \mathrm{mg} / \mathrm{l} \mathrm{TCLP}$

- D011 = concentration based standard $=0.14 \mathrm{mg} / 1$ TCLP

- D018 $=$ concentration based standard $=10 \mathrm{mg} / \mathrm{kg}$

- D040 = concentration based standard $=6 \mathrm{mg} / \mathrm{kg}$

- D001 through D043 nonwastewaters to be land disposed must be treated to meet the Universal Treatment Standards (UTS) for any underlying hazardous constituents (UHC) that may be present.

\section{Waste Characterization}

- Sampling and analysis are used to characterize the waste stream.

- Confidence level is high because sampling and analysis is available.

\section{Radiological Characterization}

- Total activity is $120 \mathrm{nCi} / \mathrm{g}$.

- $\mathrm{Cm}^{244}, \mathrm{Am}^{241}, \mathrm{Pu}^{239}, \mathrm{Eu}^{154}, \mathrm{Ba}^{137}, \mathrm{H}^{3}, \mathrm{Pu}^{241}, \mathrm{Sb}^{125}, \mathrm{Cm}^{243}, \mathrm{Pu}^{240}, \mathrm{Tc}^{99}, \mathrm{Pu}^{238}$, and $\mathrm{Cs}^{137}$; lesser amounts of $\mathrm{Zr}^{95}$, $\mathrm{Th}^{234}, \mathrm{Zn}^{65}, \mathrm{Pr} \mathrm{Pr}^{144}, \mathrm{Co}^{60}, \mathrm{Ag}^{110 m}, \mathrm{C}^{14}, \mathrm{Co}^{57}, \mathrm{Eu}^{155}, \mathrm{I}^{129}, \mathrm{Na}^{22}, \mathrm{~Pa}^{234 \mathrm{~m}}, \mathrm{Pu}^{242}, \mathrm{Se}^{79}, \mathrm{Sr}^{90}, \mathrm{U}^{233}, \mathrm{U}^{234}, \mathrm{U}^{235}, \mathrm{U}^{238}$, and $\mathrm{Y}^{90}$

- Waste is contact handled.

- Mixed low-level waste.

\subsection{Mixed Low-Level Waste Streams for Which Further Characterization is Required}

This section discusses the schedules for those specific waste streams that require further chemical or radiological characterization prior to the selection of a preferred treatment option. The categories in this section are divided into waste streams to be further characterized and hazardous wastes awaiting radiological screening.

\subsubsection{Waste Streams to be Further Characterized}

Currently, there are no waste streams in this category. The waste streams previously assigned to this category, SRW025, Solvent/TRU Job Control Waste $<100 \mathrm{nCi} / \mathrm{g}$ and SR-W033, Thirds/TRU Job Control Waste $<100 \mathrm{nCi} / \mathrm{g}$, have been relocated to Section 4.2.2 of Chapter 4 of this Volume. 


\subsubsection{Hazardous Wastes Awaiting Radiological Screening}

The preferred option for the waste streams in this waste group is the development of sampling protocols to verify that SRS has not introduced radiological contamination or analytical techniques to properly characterize the radiological constituents in the waste. Afterward, waste can be appropriately classified as mixed or hazardous only, and the proper management can be identified. Waste characterized as mixed will undergo technical analysis for treatment option identification or be placed into an existing waste treatment category.

\section{General Information}

At present, there are no wastes in this group.

\subsection{Mixed Low-Level Waste Streams Requiring Radionuclide Decay Prior to LDR Treatment}

\section{Radioactive Decay Waste Group}

The preferred treatment option for Radioactive Decay Waste Group is treatment by aging in a regulated storage facility followed by combustion and/or appropriate mercury treatment.

\section{General Information}

At the present time, the sole representative of this waste group is:

\section{SR-W036, Tritiated Oil with Mercury}

This waste stream consists of used oil from pumps and compressors operated in the tritium facilities. The oil is contaminated with tritium and mercury. Reliable characterization is hindered because of concerns about exposure of laboratory personnel to the high levels of radiation in the oil. Moreover, the radiation has the potential to cause scintillation counting interferences. The possibility of mercury contamination has been established, but the concentration has not been quantified. Greater than Class A limits per 10CFR 61.55

Volume

- Volume data for this waste stream can be found in Chapter 11.

Waste Stream Composition

- Other inorganic particulates

Waste Code

- D009E (hydraulic oil contaminated with $\mathrm{Hg}$ and radioactive materials)

- Nonwastewater

LDR Treatment Standard

- D009 $=$ Specified Technology $=$ IMERC

Waste Characterization

- Process knowledge is used to characterize the waste stream.

- Confidence level is low. High tritium levels prevent analysis. 


\section{Radiological Characterization}

- Tritium contamination variable (background to $\sim 185 \mathrm{Ci} / \mathrm{l}$ ).

- Waste is contact handled.

- Mixed low-level waste.

SR-W090, Elemental Mercury (High Rad)

This waste stream consists of used elemental mercury that has become contaminated with tritium and exhibits concentrations of tritium that exceed our current commercial vendor's acceptance criteria.

Volume

- Volume data for this waste stream can be found in Chapter 11.

Waste Stream Composition

- Other inorganic particulates

Waste Code

- D009D

LDR Treatment Standard

- AMALGM

Waste Characterization

- Process knowledge is used to characterize the waste stream.

- Confidence level is low. High tritium levels prevent analysis.

Radiological Characterization

- Tritium contamination variable

- Waste is contact handled.

- Mixed low-level waste.

\subsection{SRS Treatment Facilities that have Discontinued/Suspended Operations}

\subsubsection{Consolidated Incineration Facility (CIF)}

Combustion in CIF was the preferred option for certain mixed waste streams listed below. Previous CIF commitments and assumptions are included in the following pages for historical information.

SR-W001, Rad-Contaminated Solvents

SR-W003, Solvent Contaminated Debris (LLW)

SR-W012, Toxic Characteristics Solids for Treatment in CIF

SR-W018, Listed Incinerable Solids

SR- W022, DWPF Benzene

SR-W028, Mark 15 Filter Paper 
SR-W035, Mixed Waste Oil-Sitewide

SR-W042, Paints and Thinners

SR-W045, Tri-Butyl-Phosphate and n-Paraffin (PUREX)

SR-W051, Spent Filter Cartridges and Carbon Filter Media

SR-W055, Job Control Waste Containing Solvent Contaminated Wipes

SR-W070, Mixed Waste from Laboratory Samples

SR-W071, Wastewater Suitable for Treatment in CIF

The 1995 RCRA Permit was revised February 2, 2001. This permit revision identified that CIF operations were suspended. A decision was made April 1, 2002, to use alternative treatment for the waste streams. In accordance with the permit, progress on alternative treatment for PUREX will be summarized in an annual report to SCDHEC. See 3.2.3.

\section{Schedule for this Onsite Facility}

Submittal of all applicable permit applications:

Entering into contracts:

Initiating construction:

Conducting systems testing:

Commencing operations:

Submit waste processing schedule:
Completed. CIF received its RCRA Part B Permit with an effective date of November 1992. The air emissions construction permit was effective in December 1992. The National Emission Standards for Hazardous Air Pollutants (NESHAP) construction permit was received in June 1989.

Completed. Entering into contracts was completed prior to approval of the STP on September 29, 1995.

Completed. Construction was initiated on January 5, 1993, and essentially completed in July 1995.

Completed. Systems testing began in July 1995.

Completed. The CIF began operation on April 24, 1997.

Completed. Schedule was submitted on October 17, 1997. Schedule, as amended with this update, includes the following commitments:

Complete processing of $50 \%$ of back-logged non-PUREX SRS mixed wastes by 4QFY98. (Completed September 18, 1998. $370.9 \mathrm{~m}^{3}$ processed of a total of $702 \mathrm{~m}^{3}$.)

\subsubsection{Recycling}

Recycling in D-Area Heavy Water Facility was the preferred option for certain waste streams, including the following:

SR-W032A, Mercury Contaminated Heavy Water

Completion of treatment of SR-W032A, Mercury Contaminated Heavy Water occurred September 25, 1997, at the D-Area Heavy Water Facility, which was closed as of September 30, 1999. No additional waste is awaiting treatment. 


\subsubsection{M-Area Vendor Treatment}

Stabilization by vitrification in the M-Area Vendor Treatment Facility was the preferred option for certain mixed waste streams, including, but not limited to, the following:

SR-W005, Mark 15 Filtercake

SR-W029, M-Area Sludge Treatability Samples

SR-W031, Uranium/Chromium Solution

SR-W037, M-Area Plating Line Sludges

SR-W038, Plating Line Sump Material

SR-W039, Nickel Plating Line Solution

SR-W048, Soils from Spill Remediation

Completion of treatment of waste stream SR-W048 occurred February 8, 1999; however, additional waste meeting the description of SR-W048 has recently been generated (see Sec. 3.1.2.5 for preferred option). Completion of treatment of waste streams SR-W005, SR-W029, SR-W031, SR-W037, SR-W038, and SR-W039 occurred February 22, 1999. No additional waste is awaiting treatment, and as of October 1, 1999, the M-Area Vendor Treatment Facility underwent closure per the Clean Water Act. A small quantity of sludge generated at the time of facility closure was treated by stabilization in a 90-day accumulation area.

\section{Treatment of Onsite Wastes Requiring Vitrification}

The following new waste stream SR-W082, Radioactive Chemicals, Metals, and Pesticides (CMP) Soil, was declared a mixed waste July 7, 1998. The preferred treatment option, which was to be submitted to SCDHEC by July 30, 1999, was submitted to SCDHEC on October 16, 1998. The preferred treatment option was vitrification of the waste at the M-Area Vendor Treatment Facility. Treatment of the waste in storage at that time was completed February 24, 1999, in the M-Area Vendor Treatment Facility. Additional waste has been generated. The preferred option for this new SR-W082 waste is found in Sec. 3.1.2.5.

\subsubsection{Decontamination}

Decontamination in an existing onsite facility was the preferred option for certain mixed waste streams, including, but not limited to, the following:

SR-W013, Low-Level Waste (LLW) Lead- to be Decontaminated

Completion of treatment of SR-W013 occurred January 31, 2002, when no deconnable lead was in RCRA storage. Future deconnable lead is managed as useable material and is decontaminated on a regular basis. 


\section{Appendix for LDR Compliant Wastes}

This appendix contains descriptive information on waste streams which do not appear in the Compliance Plan Volume preferred option discussion because they meet the Land Disposal Restrictions (LDR) Treatment Standard, meet the LDR standard when they are generated, have been treated and closed as a stream, or are recycled (includes scrap metal).

\section{SR-W008, Separations Area Sample Receipts From SRNL}

This stream consists of samples returned to the canyon from SRNL.

Volume

- Volume Data for this waste stream can be found in Chapter 11.

Waste Stream Composition

- Aqueous liquid

Waste Code

- D002 (corrosive, wastewater)

- D007 (TCLP Cr)

- D008A (TCLP Pb)

- D009A (TCLP Hg)

- D018 (Benzene)

LDR Treatment Standard

- $\quad$ D002 $=$ Render non-hazardous (and ultimately manage in a CWA facility)

- $\quad$ D007 = Render non-hazardous (and ultimately manage in a CWA facility)

- $\quad$ D008A = Render non-hazardous (and ultimately manage in a CWA facility)

- $\mathrm{D} 009=$ Render non-hazardous (and ultimately manage in a CWA facility)

- $\quad$ D018 $=$ Render non-hazardous (and ultimately manage in a CWA facility)

Waste Characterization

- Sample Results

Radiological Characterization

- Alpha and beta/gamma emitters present. Tritium present.

\section{SR-W011, Cadmium-Coated HEPA Filters}

This waste stream consists of HEPA filter frames used to filter ventilation air from the reactors. This material is exempt from RCRA under the scrap metal exclusion (Part 261.6(a)(3)0. The scrap metal has been recycled by a vendor. The filter frames are hazardous due to cadmium plating on the metal frames. Replacement units are stainless steel framed filters. 
Volume

- Volume Data for this waste stream can be found in Chapter 11.

Waste Stream Composition

- Cadmium-containing metal debris

Waste Code

- D006A (TCLP Cd) nonwastewater

LDR Treatment Standard

- N/A - Scrap Metal Exclusion

Waste Characterization

- Sample Results

Radiological Characterization

- $\quad$ Tritium is present

\section{SR-W015, Mercury/Tritium Contaminated Equipment}

Retired process equipment (mostly pumps) contaminated with tritium, sometimes mercury (HWI \#002), sometimes lead (HWI \#033), and sometimes silver (HWI \#0057).

Retired equipment has both adsorbed and matrix-diffused tritium contamination and wetted-surface mercury residues from direct contact with process gases. Lead is present in some of the equipment (fuel cells, solder and lead collars).

Volume

- Data for this waste stream can be found in Chapter 11.

Waste Stream Composition

- Predominantly metal debris

Waste Code

- D008A (TCLP Pb)

- D009A (TCLP Hg)

- D011 (TCLP Ag)

LDR Treatment Standard

- $\quad$ D008A $=$ concentration based standard $=0.75 \mathrm{mg} / \mathrm{l}$ TCLP

- $\mathrm{D} 009 \mathrm{~A}=$ concentration based standard $=0.025 \mathrm{mg} / \mathrm{l}$ TCLP

- D011 $=$ concentration based standard $=0.14 \mathrm{mg} / \mathrm{l} \mathrm{TCLP}$ 


\section{Waste Characterization}

- Process Knowledge

Radiological Characterization

- $\mathrm{U}^{238}$ and Tritium present

\section{SR-W020, Salt Processing Filters}

These filters may be a future debris waste stream. The filters are used in the treatment and separation of salt solution in preparation for processing in the Defense Waste Processing Facility (DWPF) and Saltstone Facility. The salt solution is treated to remove radioactive cesium and to adsorb strontium and plutonium. This process is expected to eventually foul the filters, requiring their removal, treatment, and disposal.

\section{Volume}

- Volume data for this waste stream can be found in Chapter 11.

\section{Waste Stream Composition}

- Inorganic debris

Waste Code

- D009A (TCLP Hg)

- D018 (Benzene)

- D036 (Nitrobenzene)

- Nonwastewater

LDR Treatment Standard

- $\mathrm{D} 009=$ concentration based standard $=0.025 \mathrm{mg} / \mathrm{l}$ TCLP

- D018 $=$ concentration based standard $=10 \mathrm{mg} / \mathrm{kg}$

- D036 = concentration based standard $=14 \mathrm{mg} / \mathrm{kg}$

- Alternate debris technology may be applied.

- D001 through D043 nonwastewaters to be land disposed must be treated to meet the Universal Treatment Standards (UTS) for any underlying hazardous constituents (UHC) that may be present.

\section{Waste Characterization}

- Process knowledge is used to characterize the waste stream.

- Confidence level is medium since this waste stream has not yet been generated.

- Typical expected concentration is $236 \mathrm{~g} \mathrm{Hg}$ and $5000 \mathrm{~g}$ benzene per filter. This is estimated by calculation.

\section{Radiological Characterization}

- Total activity is estimated to be $64-3400 \mathrm{Ci} /$ filter.

- Beta/gamma emitters are $\mathrm{Cs}^{137}, \mathrm{Cs}^{134}, \mathrm{Sr}^{90}, \mathrm{Tc}^{99}, \mathrm{Ru}^{106}, \mathrm{Sb}^{125}$, and $\mathrm{I}^{129}$.

- Waste is remote handled.

- Mixed low-level waste 


\section{SR-W023, Cadmium Safety/Control Rods}

The safety/control rods are steel encapsulated, cadmium containing rods used to control neutron flux in the reactors. The rods are approximately 22 feet long, one inch in diameter. The Cadmium Rods are macroencapsulated by being placed in a stainless or mild steel container that is welded closed. One rod was cut and placed in a drum; other rods were not cut prior to placement in a box.

\section{Volume}

- Volume Data for this waste stream can be found in Chapter 11.

Waste Stream Composition

- Cadmium-containing metal debris

Waste Code

- D006A (TCLP Cd)

LDR Treatment Standard

- $\quad$ D006A $=$ concentration based standard $=0.11 \mathrm{mg} / \mathrm{l} \mathrm{TCLP}$

- D001 through D043 nonwastewaters to be land disposed must be treated to meet Universal Treatment Standards (UTS) for any underlying hazardous constituents (UHC) that may be present.

Waste Characterization

- Sample Results

Radiological Characterization

- Beta/gamma emitters present

\section{SR-W024, Mercury/Tritium Gold Traps}

Elemental mercury has been amalgamated on gold foil. A typical trap consists of a stainless steel cylindrical housing that is 38 inches high and 2 inches in diameter that contains gold foil on 16 evenly spaced trays. Each trap contains $\sim 125 \mathrm{~g}$ of elemental gold. Contamination is variable, depending on use and changeout frequency. Retired traps typically show surface amalgamation on $\sim 1 / 4$ of the gold, and contain $0.6 \mathrm{~g}$ of bound mercury. The estimated tritium contamination is $200 \mathrm{Ci}$ per trap and is deemed conservative.

\section{Volume}

- Volume Data for this waste stream can be found in Chapter 11.

Waste Stream Composition

- Metal debris

Waste Code

- D009A (TCLP Hg) 


\section{LDR Treatment Standard}

- $\quad$ D009A $=$ concentration based standard $=0.025 \mathrm{mg} / \mathrm{l}$ TCLP

- D001 through D043 nonwastewaters to be land disposed must be treated to meet Universal Treatment Standards (UTS) for any underlying hazardous constituents (UHC) that may be present.

Waste Characterization

- Process knowledge

Radiological Characterization

- $\quad$ Tritium is present

\section{SR-W032B, Mercury-Contaminated Heavy Water Residues}

Heavy Water Operations laboratory in D area generated waste stream SR-W032A, "Mercury-Contaminated Heavy Water" during analytical testing using mercuric chloride. The flush water from this analysis was collected and managed as hazardous waste. That waste stream is no longer generated due to a change in analysis procedures. The mercury content is now below toxic limits. SR-W032B is the residues from treatment of SR-W032A.

Volume

- Volume Data for this waste stream can be found in Chapter 11.

Waste Stream Composition

- Nonwastewater

Waste Code

- D009A (TCLP Hg)

LDR Treatment Standard

- $\quad \mathrm{D} 009 \mathrm{~A}=$ concentration based standard $=0.025 \mathrm{mg} / \mathrm{l} \mathrm{TCLP}$

- D001 through D043 nonwastewaters to be land disposed must be treated to meet Universal Treatment Standards (UTS) for any underlying hazardous constituents (UHC) that may be present.

Waste Characterization

- Sample results

Radiological Characterization

- $\quad$ Tritium is present

\section{SR-W040, Listed Stabilized Sludge/Listed LDR-Compliant Debris}

This waste is a stabilized sludge generated from the treatment of nickel plating line waste water and is stored in the M-Area pad. Also includes equipment, associated with the vitrification treatment process, which meet LDR. This includes an approximately 90,000 $\mathrm{lb}$ (gross weight) melter $(14.3 \mathrm{~m} 3)$ full of treated F006 waste and small amounts 
of debris/spill material which meets the LDR standards. The stream will also include debris items, meeting LDR, that were generated by CIF operations or RCRA listed contaminated soil.

\section{Volume}

- Volume Data for this waste stream can be found in Chapter 11.

Waste Stream Composition

- $\quad$ Stabilized sludge and miscellaneous debris

Waste Code

- F001-F003, F005 (halogenated and non-halogenated spent solvents)

- F006 (wastewater treatment sludges from electroplating operations)

- F028 (residues from thermal treatment of F020, F021, F026, and F027 wastes)

- U002 (Acetone)

- U003 (Acetonitrile)

- U019 (Benzene)

- U037 (Chlorobenzene)

- U080 (Methylene chloride)

- U123 (Formic acid)

- U127 (Hexachlorobenzene)

- U131 (Hexachloroethane)

- U159 (Methyl ethyl ketone)

- U165 (Napthalene)

- U188 (Phenol)

- U210 (Tetrachloroethylene)

- U211 (Carbon tetrachloride)

- U220 (Toluene)

- $\mathrm{U} 226$ (1,1,1-Trichloroethane)

- U228 (Trichloroethylene)

- U239 (Xylenes)

\section{LDR Treatment Standard}

- $\quad \mathrm{F} 001$ and F002 $=$ concentration based standard $=6.0-30 \mathrm{mg} / \mathrm{kg}$

- $\mathrm{F} 003=$ concentration based standard $=0.75 \mathrm{mg} / \mathrm{l}$, TCLP $-160 \mathrm{mg} / \mathrm{kg}$

- $\mathrm{F} 005=$ concentration based standard $=4.8 \mathrm{mg} / \mathrm{l}$, TCLP $-170 \mathrm{mg} / \mathrm{kg}$ except 2-Ethoxyethanol and 2Nitropropane $=$ CMBST

- $\mathrm{F} 006=$ concentration based standard $=0.11-11 \mathrm{mg} / \mathrm{l} \mathrm{TCLP}$

- $\mathrm{F} 028==$ concentration based standard $==0.001$ to $7.4 \mathrm{mg} / \mathrm{kg}$

- $\mathrm{U} 002=$ concentration based standard $=160 \mathrm{mg} / \mathrm{kg}$

- $\mathrm{U} 003=$ concentration based standard $=38 \mathrm{mg} / \mathrm{kg}$ or CMBST

- $\mathrm{U} 019=$ concentration based standard $=10 \mathrm{mg} / \mathrm{kg}$

- $\mathrm{U} 037=$ concentration based standard $=6.0 \mathrm{mg} / \mathrm{kg}$

- $\mathrm{U} 080=$ concentration based standard $=30 \mathrm{mg} / \mathrm{kg}$

- $\mathrm{U} 123=$ specified technology $=$ CMBST

- $\mathrm{U} 127=$ concentration based standard $=10 \mathrm{mg} / \mathrm{kg}$

- $\mathrm{U} 131=$ concentration based standard $=30 \mathrm{mg} / \mathrm{kg}$

- $\mathrm{U} 165=$ concentration based standard $=5.6 \mathrm{mg} / \mathrm{kg}$

- $\mathrm{U} 188=$ concentration based standard $=6.2 \mathrm{mg} / \mathrm{kg}$

- $\mathrm{U} 210=$ concentration based standard $=6.0 \mathrm{mg} / \mathrm{kg}$

- $\mathrm{U} 211=$ concentration based standard $=6.0 \mathrm{mg} / \mathrm{kg}$ 
- $\mathrm{U} 220=$ concentration based standard $=10.0 \mathrm{mg} / \mathrm{kg}$

- $\mathrm{U} 226=$ concentration based standard $=6.0 \mathrm{mg} / \mathrm{kg}$

- $\mathrm{U} 228=$ concentration based standard $=6.0 \mathrm{mg} / \mathrm{kg}$

- $\mathrm{U} 239=$ concentration based standard $=30 \mathrm{mg} / \mathrm{kg}$

Waste Characterization

- Sample results

Radiological Characterization

- Alpha and beta/gamma emitters present

\section{SR-W041, Aqueous Mercury and Lead}

Six small (e.g., 2 liters, 4 liters, etc.) containers of aqueous waste with lead and mercury overpacked in a poly-lined $55 \mathrm{gal}$. drum. An additional 55 gallon drum in this waste stream is rinsate from the rinsing of Tank E-3-1 which contains low levels of mercury.

Volume

- Volume Data for this waste stream can be found in Chapter 11.

Waste Stream Composition

- Aqueous Liquid

Waste Code

- $\quad$ D008A (TCLP Pb)

- D009A (TCLP Hg)

- D009C (high Hg contains inorganics) wastewater

LDR Treatment Standard

- $\quad$ D008 $=$ concentration based standard $=0.75 \mathrm{mg} / \mathrm{l} \mathrm{TCLP}$

- $\mathrm{D} 009=$ concentration based standard $=0.20 \mathrm{mg} / \mathrm{l} \mathrm{TCLP}$; or RMERC

- D001 through D043 nonwastewaters to be land disposed must be treated to meet Universal Treatment Standards (UTS) for any underlying hazardous constituents (UHC) that may be present.

Waste Characterization

- Sample Results

Radiological Characterization

- Alpha emitters, beta/gamma emitters, and tritium are present.

SR-W046, Consolidated Incineration Facility (CIF) Ash

SR-W047, Consolidated Incineration Facility (CIF) Blowdown

These streams were stabilized using the CIF Ashcrete/Blowcrete unit and are not currently generated, with CIF in suspension of operation 
The following information is applicable to both the waste streams in this waste group.

Volume

- Volume data for this waste stream can be found in Chapter 11.

Waste Stream Composition

- SR-W046 - Inorganic sludge/particulate

- SR-W047 - Aqueous liquid and solids

Waste Code

- D004 (TCLP As)

- D005 (TCLP Ba)

- D006A (TCLP Cd)

- D007 (TCLP Cr)

- D008A (TCLP Pb)

- D009A (TCLP Hg)

- D010 (TCLP Se)

- D011 (TCLP Ag)

- D012 (Endrin)

- D013 (Lindane)

- D014 (Methoxyclor)

- D015 (Toxaphene)

- D016 (2,4-D)

- D017 (2,4,5-TP/Silvex)

- D018 (Benzene)

- D019 (Carbon Tetrachloride)

- D020 (Chlordane)

- D021 (Chlorobenzene)

- D022 (Chloroform)

- D023 (o-Cresol)

- D024 (m-Cresol)

- D025 (p-Cresol)

- D026 (Cresol - total)

- D027 (1,4-Dichlorobenzene)

- D028 (1,2-Dichloroethane)

- D029 (1,2-Dichloroethylene)

- D030 (2,4-Dinitrotoluene)

- D031 (Heptachlor)

- D032 (Hexachlorobenzene)

- D033 (Hexachlorobutadiene)

- D034 (Hexachloroethane)

- D035 (Methyl Ethyl Ketone)

- D036 (Nitrobenzene)

- D037 (Pentachlorophenol)

- D038 (Pyridine)

- D039 (Tetrachloroethylene)

- D040 (Trichloroethylene)

- D041 (2,4,5-Trichlorophenol)

- D042 (2,4,6-Trichlorophenol)

- D043 (Vinyl Chloride)

- F001 (Unspecified solvents)

- F002 (Unspecified solvents)

- F003X (Unspecified solvents)

- F004X (Unspecified solvents
- U108 (1,4-Dioxane)

- U109 (1,2-Diphenylhydrazine)

- U110 (Dipropylamine)

- U112 (Ethyl acetate)

- U115 (Ethylene oxide)

- U116 (Ethylene thiourea)

- U117 (Ethyl ether)

- U121 (Trichloromonofluoromethane)

- U122 (Formaldehyde)

- U123 (Formic acid)

- U124 (Furan)

- U125 (Furfural)

- U127 (Hexachlorobenzene)

- U128 (Hexachlorobutadiene)

- U129 (Lindane)

- U130 (Hexachlorocyclopentadiene)

- U131 (Hexachloroethane)

- U132 (Hexachlorophene)

- U133 (Hydrazine)

- U134 (Hydrogen fluoride)

- U136 (Cacodylic acid)

- U138 (Iodomethane)

- U140 (Isobutyl alcohol)

- U142 (Kepone)

- U144 (Lead acetate)

- U146 (Lead subacetate)

- U147 (Maleic anhydride)

- U151 (Mercury wastewaters)

- U154 (Methanol)

- U157 (3-Methylcholanthrene)

- U158 (4,4'-Methylene bis(2-chloroaniline)

- U159 (Methyl ethyl ketone)

- U161 (Methyl isobutyl ketone)

- U162 (Methyl methacrylate)

- U165 (Naphthalene)

- U166 (1,4-Naphthoquinone)

- U167 (1-Naphthylamine)

- U169 (Nitrobenzene)

- U170 (p-Nitrophenol)

- U171 (2-Nitropropane)

- U182 (Paraldehyde)

- U183 (Pentachlorobenzene)

- U184 (Pentachloroethane)

- U185 (Pentachloronitrobenzene) 
- F005X (Unspecified solvents)

- F006 (Metal plating waste without cyanide)

- U001 (Acetaldehyde)

- U002 (Acetone)

- U003 (Acetonitrile)

- U004 (Acetophenone)

- U006 (Acetyl chloride)

- U007 (Acrylamide)

- U008 (Acrylic Acid)

- U009 (Acrylonitrile)

- U011 (Amitrole)

- U012 (Aniline)

- U014 (Auramine)

- U017 (Benzal chloride)

- U019 (Benzene)

- U021 (Benzidine)

- U022 (Benzo(a)pyrene)

- U023 (Benzotrichloride)

- U025 (bis(2-Chloroethoxy)methane)

- U027 (bix(2-Chloroisopropyl)ether)

- U028 (bis(2-Ethylhexyl) phthalate)

- U031 (n-Butyl alcohol)

- U032 (Calcium chromate)

- U034 (Trichloroacetaldehyde)

- U036 (Chlordane)

- U037 (Chlorobenzene)

- U041 (Epichlorohydrin)

- U043 (Vinyl chloride)

- U044 (Chloroform)

- U045 (Methyl chloride)

- U046 (Chloromethyl methyl ether)

- U047 (2-Chloronaphthalene)

- U048 (2-Chlorophenol)

- U050 (Chrysene)

- U051 (Creosote)

- U052 (Creosols)

- U053 (Crotonaldehyde)

- U055 (Cumene)

- U056 (Cyclohexane)

- U057 (Cyclohexanone)

- U060 (DDD)

- U061 (DDT)

- U063 (Dibenz(a,h)anthracene)

- U067 (Etheylene dibromide)

- U069 (Di-n-buthyl phthalate)

- U070 (o-Dichlorobenzene)

- U071 (m-Dichlorobenzene)

- U072 (p-Dichlorobenzene)

- U073 (3,3'-Dechlorobenzidine)

- U074 (1,4-Dichloro-2-butene)

- U075 (Dichlorodifluoromethane)

- U076 (1,1-Dichloroethane)

- U077 (1,2-Dichloroethane)

- U078 (1,1-Dichloroethylene)

- U079 (1,2-Dichloroethylene)

- U080 (Methylene chloride)

- U081 (2,4-Dichlorophenol)
- U187 (Phenacetin)

- U188 (Phenol)

- U190 (Phthalic anhydride)

- U196 (Pyridine)

- U197 (p-Benzoquinone)

- U201 (Resorcinol)

- U204 (Selenium dioxide)

- U207 (1,2,4,5-Tetrachlorobenzene)

- U208 (1,1,1,2-Tetrachloroethane)

- U209 (1,1,2,2-Tetrachloroethane)

- U210 (Tetrachloroethylene)

- U211 (Carbon Tetrachloride)

- U213 (Tetrahydrofuran)

- U215 (Thallium (I) carbonate)

- U216 (Thallium (I) chloride)

- U218 (Thioacetamide)

- U219 (Thiourea)

- U220 (Toluene)

- U223 (Toluene diisocyanate)

- U225 (Bromoform)

- U226 (1,1,1-Trichloroethane)

- U227 (1,1,2-Trichloroethane)

- U228 (Trichloroethylene)

- U234 (1,3,5-Trinitrobenzene)

- U236 (Trypan Blue)

- U239 (Xylenes)

- U247 (Methyoxyclor)

- U328 (o-Toluidine)

- U359 (2-Ethoxyethanol)

- P005 (Allyl alcohol)

- P009 (Ammonium picrate)

- P011 (Arsenic pentoxide)

- P012 (Arsenic trioxide)

- P016 (Dichloromethyl ether)

- P017 (Bromoacetone)

- P018 (Brucine)

- P022 (Carbon disulfide)

- P023 (Chloroacetaldehyde)

- P024 (p-Chloroaniline)

- P028 (Benzyl chloride)

- P037 (Dieldrin)

- P045 (Thiofanox)

- P048 (2,4-Dinitrophenol)

- P051 (Endrin)

- P054 (Aziridine)

- P059 (Heptaclor)

- P060 (Isodrin)

- P063 (Hydrogen cyanide)

- P064 (Isocyanic acid, ethyl ester)

- P068 (Methyl hydrrazine)

- P069 (2-Methyllactonitrile)

- P070 (Aldicarb)

- P077 (p-Nitroaniline)

- P089 (Parathion)

- P093 (Phenylthiourea)

- P095 (Phosgene)

- P098 (Potassium cyanide) 
- U082 (2,6-Dichlorophenol)

- U083 (1,2-Dichloroproane)

- U084 (1,3-Dichloropropylene)

- U088 (Diethyl phthalate)

- U091 (3,3-Dimethoxybenzidine)

- U092 (Dimethylamine)

- U095 (3,3'-Dimethylbenzidine)

- U102 (Dimethyl phthalate)

- U103 (Dimethyl sulfate)

- U105 (2,4-Dinitrotoluene)

- U106 (2,6-Dinitrotoluene)
- P102 (Propargyl alcohol)

- P104 (Silver cyanide)

- P105 (Sodium azide)

- P106 (Sodium cyanide)

- P108 (Strychnine and salts)

- P112 (Tetranitromethane)

- P113 (Thallic oxide)

- P116 (Thiosemicarbazide)

- P119 (Ammonium vanadate)

- P120 (Vanadium pentoxide)

- P123 (Toxaphene)

- The waste codes describing the CIF ash and blowdown waste streams depend on the feed stream into CIF. The Blowdown waste stream will contain all of the listed waste codes that were fed into the CIF.

\section{LDR Treatment Standard}

- D004 = concentration based standard $=5.0 \mathrm{mg} / \mathrm{l}$, TCLP

- D005 = concentration based standard $=21 \mathrm{mg} / \mathrm{l}, \mathrm{TCLP}$

- D006 = concentration based standard $=0.11 \mathrm{mg} / \mathrm{l}, \mathrm{TCLP}$

- D007 = concentration based standard $=0.60 \mathrm{mg} / \mathrm{l}$, TCLP

- D008 = concentration based standard $=0.75 \mathrm{mg} / \mathrm{l}$, TCLP

- $\mathrm{D} 009=$ concentration based standard $=0.025 \mathrm{mg} / \mathrm{l}$, TCLP

- $\mathrm{D} 010=$ concentration based standard $=5.7 \mathrm{mg} / \mathrm{l}$, TCLP

- D011 = concentration based standard $=0.14 \mathrm{mg} / \mathrm{l}$, TCLP

- $\mathrm{D} 012=$ concentration based standard $=0.13 \mathrm{mg} / \mathrm{kg}$

- $\mathrm{D} 013=$ concentration based standard $=0.0166 \mathrm{mg} / \mathrm{kg}$

- D014 = concentration based standard $=0.18 \mathrm{mg} / \mathrm{kg}$

- D015 = concentration based standard $=2.6 \mathrm{mg} / \mathrm{kg}$

- D016 = concentration based standard $=10 \mathrm{mg} / \mathrm{kg}$

- $\mathrm{D} 017=$ concentration based standard $=7.9 \mathrm{mg} / \mathrm{kg}$

- $\mathrm{D} 018=$ concentration based standard $=10 \mathrm{mg} / \mathrm{kg}$

- D019, D039, \& D040 = concentration based standard $=6.0 \mathrm{mg} / \mathrm{kg}$

- $\mathrm{D} 020=$ concentration based standard $=0.26 \mathrm{mg} / \mathrm{kg}$

- D021 \& D022 = concentration based standard $=6.0 \mathrm{mg} / \mathrm{kg}$

- D023, D024 \& D025 = concentration based standard = 5.6 mg/kg

- D026 = concentration based standard $=11.2 \mathrm{mg} / \mathrm{kg}$

- D027, D028, D029, \& D043 = concentration based standard $=6.0 \mathrm{mg} / \mathrm{kg}$

- D030 = concentration based standard $=140 \mathrm{mg} / \mathrm{kg}$

- $\mathrm{D} 031=$ concentration based standard $=0.066 \mathrm{mg} / \mathrm{kg}$

- $\mathrm{D} 032=$ concentration based standard $=10 \mathrm{mg} / \mathrm{kg}$

- D033 = concentration based standard $=5.6 \mathrm{mg} / \mathrm{kg}$

- D034 = concentration based standard $=30 \mathrm{mg} / \mathrm{kg}$

- $\mathrm{D} 035=$ concentration based standard $=36 \mathrm{mg} / \mathrm{kg}$

- $\mathrm{D} 036=$ concentration based standard $=14 \mathrm{mg} / \mathrm{kg}$

- D037, D041, \& D042 = concentration based standard = $7.4 \mathrm{mg} / \mathrm{kg}$

- $\mathrm{D} 038=$ concentration based standard $=16 \mathrm{mg} / \mathrm{kg}$

- $\mathrm{F} 001=$ concentration based standard $=6-30 \mathrm{mg} / \mathrm{kg}$

- $\mathrm{F} 002=$ concentration based standard $=6-30 \mathrm{mg} / \mathrm{kg}$

- $\mathrm{F} 003=$ concentration based standard $=2.6-60 \mathrm{mg} / \mathrm{kg}$

- $\mathrm{F} 005=$ concentration based standard $=10-170 \mathrm{mg} / \mathrm{kg}$

- $\mathrm{F} 006=$ concentration based standard $=0.19-5.0 \mathrm{mg} / \mathrm{l}$, TCLP

- U001, U006, U007, \& U008 = specified technology = CMBST

- $\mathrm{U} 002=$ concentration based standard $=160 \mathrm{mg} / \mathrm{kg}$

- U003, U011, U014, \& U017 = specified technology = CMBST

- $\mathrm{U} 004=$ concentration based standard $=9.7 \mathrm{mg} / \mathrm{kg}$

- $\mathrm{U} 009=$ concentration based standard $=84 \mathrm{mg} / \mathrm{kg}$

- $\mathrm{U} 012=$ concentration based standard $=14 \mathrm{mg} / \mathrm{kg}$ 
- $\mathrm{U} 019=$ concentration based standard $=10 \mathrm{mg} / \mathrm{kg}$

- U021, U034, U041, \& U046 = specified technology = CMBST

- $\mathrm{U} 022=$ concentration based standard $=3.4 \mathrm{mg} / \mathrm{kg}$

- U023, U103, \& U109 = specified technology = CHOXD; CHRED; or CMBST

- U025, U070, U071, \& U072 = concentration based standard $=6.0 \mathrm{mg} / \mathrm{kg}$

- $\mathrm{U} 027=$ concentration based standard $=7.2 \mathrm{mg} / \mathrm{kg}$

- $\mathrm{U} 028=$ concentration based standard $=28 \mathrm{mg} / \mathrm{kg}$

- $\mathrm{U} 031=$ concentration based standard $=2.6 \mathrm{mg} / \mathrm{kg}$

- $\mathrm{U} 032=$ concentration based standard $=0.60 \mathrm{mg} / \mathrm{L}$, TCLP

- $\mathrm{U} 036=$ concentration based standard $=0.26 \mathrm{mg} / \mathrm{kg}$

- U037, U043, \& U044 = concentration based standard $=6.0 \mathrm{mg} / \mathrm{kg}$

- $\mathrm{U} 045=$ concentration based standard $=30 \mathrm{mg} / \mathrm{kg}$

- $\mathrm{U} 047=$ concentration based standard $=5.6 \mathrm{mg} / \mathrm{kg}$

- $\mathrm{U} 048=$ concentration based standard $=5.7 \mathrm{mg} / \mathrm{kg}$

- $\mathrm{U} 050=$ concentration based standard $=3.4 \mathrm{mg} / \mathrm{kg}$

- $\mathrm{U} 051=$ concentration based standard $=5.6-30 \mathrm{mg} / \mathrm{kg}$

- $\mathrm{U} 052=$ concentration based standard $=5.6-11.2 \mathrm{mg} / \mathrm{kg}$

- U053, U055, \& U056= specified technology = CMBST

- U057 \& U154 = specified technology = CMBST; or for nonwastewaters $-0.75 \mathrm{mg} / \mathrm{L}, \mathrm{TCLP}$

- U060 \& D061 = concentration based standard $=0.0087 \mathrm{mg} / \mathrm{kg}$

- $\mathrm{U} 063=$ concentration based standard $=8.2 \mathrm{mg} / \mathrm{kg}$

- $\mathrm{U} 067=$ concentration based standard $=15 \mathrm{mg} / \mathrm{kg}$

- U069, U088, U102, \& U106= concentration based standard $=28 \mathrm{mg} / \mathrm{kg}$

- $\mathrm{U} 073 \& \mathrm{U} 074=$ specified technology $=$ CMBST

- $\mathrm{U} 075=$ concentration based standard $=7.2 \mathrm{mg} / \mathrm{kg}$

- U076, U077, \& U078 = concentration based standard $=6.0 \mathrm{mg} / \mathrm{kg}$

- U079 \& U080 = concentration based standard $=30 \mathrm{mg} / \mathrm{kg}$

- U081 \& U082 = concentration based standard $=14 \mathrm{mg} / \mathrm{kg}$

- U083 \& U084 = concentration based standard $=18 \mathrm{mg} / \mathrm{kg}$

- U091, U092, U095, \& U108 = specified technology = CMBST

- $\mathrm{U} 105=$ concentration based standard $=140 \mathrm{mg} / \mathrm{kg}$

- U110, U116, \& U122 = specified technology = CMBST

- $\mathrm{U} 112=$ concentration based standard $=33 \mathrm{mg} / \mathrm{kg}$

- $\mathrm{U} 115=$ specified technology $=$ CHOXD; or CMBST

- $\mathrm{U} 117=$ concentration based standard $=160 \mathrm{mg} / \mathrm{kg}$

- U121 \& U131 = concentration based standard $=30 \mathrm{mg} / \mathrm{kg}$

- U123, U124, U125, \& U132 = specified technology = CMBST

- $\mathrm{U} 127=$ concentration based standard $=10 \mathrm{mg} / \mathrm{kg}$

- $\mathrm{U} 128=$ concentration based standard $=5.6 \mathrm{mg} / \mathrm{kg}$

- $\mathrm{U} 129=$ concentration based standard $=0.066 \mathrm{mg} / \mathrm{kg}$

- $\mathrm{U} 130=$ concentration based standard $=2.4 \mathrm{mg} / \mathrm{kg}$

- $\mathrm{U} 133$ = specified technology = CHOXD; CHRED; or CMBST

- $\mathrm{U} 134=$ specified technology = ADGAS fb NEUTR; or NEUTR

- $\mathrm{U} 136=$ concentration based standard $=5.0 \mathrm{mg} / \mathrm{L}, \mathrm{TCLP}$

- $\mathrm{U} 138=$ concentration based standard $=65 \mathrm{mg} / \mathrm{kg}$

- $\mathrm{U} 140=$ concentration based standard $=170 \mathrm{mg} / \mathrm{kg}$

- $\mathrm{U} 142=$ concentration based standard $=0.13 \mathrm{mg} / \mathrm{kg}$

- U144 \& U146 = concentration based standard $=0.75 \mathrm{mg} / \mathrm{L}$, TCLP

- U147, U166, U167, \& U171 = specified technology = CMBST

- $\mathrm{U} 151=$ specified technology $=$ RMERC

- $\mathrm{U} 157=$ concentration based standard $=15 \mathrm{mg} / \mathrm{kg}$

- $\mathrm{U} 158=$ concentration based standard $=30 \mathrm{mg} / \mathrm{kg}$

- $\mathrm{U} 159=$ concentration based standard $=36 \mathrm{mg} / \mathrm{kg}$

- $\mathrm{U} 161=$ concentration based standard $=33 \mathrm{mg} / \mathrm{kg}$

- $\mathrm{U} 162=$ concentration based standard $=160 \mathrm{mg} / \mathrm{kg}$

- $\mathrm{U} 165=$ concentration based standard $=5.6 \mathrm{mg} / \mathrm{kg}$

- U169, U207 = concentration based standard $=14 \mathrm{mg} / \mathrm{kg}$ 
- $\mathrm{U} 170=$ concentration based standard $=29 \mathrm{mg} / \mathrm{kg}$

- U182, U197, U201, \& U213 = specified technology = CMBST

- $\mathrm{U} 183=$ concentration based standard $=10 \mathrm{mg} / \mathrm{kg}$

- $\mathrm{U} 184=$ specified technology = CMBST; or $6.0 \mathrm{mg} / \mathrm{kg}$

- $\mathrm{U} 185=$ concentration based standard $=4.8 \mathrm{mg} / \mathrm{kg}$

- U187, U196 = concentration based standard $=16 \mathrm{mg} / \mathrm{kg}$

- $\mathrm{U} 188=$ concentration based standard $=6.2 \mathrm{mg} / \mathrm{kg}$

- $\mathrm{U} 190=$ concentration based standard $=28 \mathrm{mg} / \mathrm{kg}$

- U204 = concentration based standard $=5.7 \mathrm{mg} / \mathrm{L}$, TCLP

- U208, U209, U210, \& U211= concentration based standard $=6.0 \mathrm{mg} / \mathrm{kg}$

- $\mathrm{U} 215$ \& U216 = specified technology = RTHRM; or STABL

- U218, U219, U223, U234, \& U236 = specified technology = CMBST

- $\mathrm{U} 220=$ concentration based standard $=10 \mathrm{mg} / \mathrm{kg}$

- $\mathrm{U} 225=$ concentration based standard $=15 \mathrm{mg} / \mathrm{kg}$

- U226, U227, \& U228 = concentration based standard $=6.0 \mathrm{mg} / \mathrm{kg}$

- $\mathrm{U} 239=$ concentration based standard $=30 \mathrm{mg} / \mathrm{kg}$

- $\mathrm{U} 247=$ concentration based standard $=0.18 \mathrm{mg} / \mathrm{kg}$

- U328 \& U359= specified technology $=$ CMBST

- P005, P016, P017, \& P018 = specified technology = CMBST

- P009, P068, P105, \& P112 = specified technology = CHOXD; CHRED; or CMBST

- P011 \& P012= concentration based standard = 5.0 mg/L, TCLP

- $\mathrm{P} 022=$ specified technology $=\mathrm{CMBST}$; or for nonwastewaters $-4.8 \mathrm{mg} / \mathrm{L}$, TCLP

- P023, P028, P045, \& P054 = specified technology = CMBST

- $\mathrm{P} 024=$ concentration based standard $=16 \mathrm{mg} / \mathrm{kg}$

- $\mathrm{P} 037=$ concentration based standard $=0.13 \mathrm{mg} / \mathrm{kg}$

- $\mathrm{P} 048=$ concentration based standard $=160 \mathrm{mg} / \mathrm{kg}$

- $\mathrm{P} 051=$ concentration based standard $=0.13 \mathrm{mg} / \mathrm{kg}$

- $\mathrm{P} 059, \mathrm{P} 060=$ concentration based standard $=0.066 \mathrm{mg} / \mathrm{kg}$

- P063, P098, \& P106= concentration based standard $=30-590 \mathrm{mg} / \mathrm{kg}$

- P064, P069, P070, \& P093 = specified technology = CMBST

- $\mathrm{P} 077=$ concentration based standard $=28 \mathrm{mg} / \mathrm{kg}$

- $\mathrm{P} 089=$ concentration based standard $=4.6 \mathrm{mg} / \mathrm{kg}$

- $\mathrm{P} 095, \mathrm{P} 102, \mathrm{P} 108, \&$ P116 = specified technology $=$ CMBST

- P104 = concentration based standard $=30-590 \mathrm{mg} / \mathrm{kg}$ for cyanides; $0.14 \mathrm{mg} / \mathrm{L}$, TCLP for silver

- P113 = specified technology = RTHRM; or STABL

- $\mathrm{P} 119 \& \mathrm{P} 120=$ specified technology $=\mathrm{STABL}$

- $\mathrm{P} 123=$ concentration based standard $=2.6 \mathrm{mg} / \mathrm{kg}$

- D001 through D043 nonwastewaters to be land disposed must be treated to meet Universal Treatment Standards (UTS) for any underlying hazardous constituents (UHC) that may be present.

\section{Waste Characterization}

- Analytical data is used to characterize these waste streams.

- Confidence level is high based on analytical data.

\section{Radiological Characterization}

- Tritium is present.

- Alpha and beta/gamma emitters are present.

- Waste is contact handled.

- Mixed low-level waste

\section{SR-W050, Waste to support High-Level Waste (HLW) Processing Demonstrations}

The waste will be generated by laboratory research, development, and analytical programs at 773-A to support the operations of the Defense Waste Processing Facility (DWPF). 
This waste stream will be generated by shielded cell operations during projects for the glass technology group and will come from demonstrations of the DWPF and IWT processes on actual HLW samples.

The liquid waste will most likely contain mercury and chromium. The chromium will most likely be at low levels, below TCLP.

Volume

- Volume Data for this waste stream can be found in Chapter 11.

Waste Stream Composition

- Aqueous slurry

Waste Code

- $\quad$ D007 (Chromium)

- D009A (TCLP Hg)

- D018 (Benzene)

LDR Treatment Standard

- $\quad$ D007 $=$ concentration based standard $=0.60 \mathrm{mg} / \mathrm{l}$ TCLP

- $\mathrm{D} 009=$ concentration based standard $=0.20 \mathrm{mg} / \mathrm{l} \mathrm{TCLP}$; or RMERC

- $\mathrm{D} 018=$ concentration based standard $=10 \mathrm{mg} / \mathrm{kg}$

- D001 through D043 nonwastewaters to be land disposed must be treated to meet Universal Treatment Standards (UTS) for any underlying hazardous constituents (UHC) that may be present.

Waste Characterization

- Sample results and process knowledge

Radiological Characterization

- Beta/gamma emitters are present.

\section{SR-W058, Mixed Sludge Waste with Mercury from DWPF Treatability Studies}

This waste stream consisted of small amounts of high-level waste supernate, sludge, and salt samples from the tank farm and mercury contamination generated during DWPF treatability studies. The waste mercury sludge dried and caked onto eight centrifuge tubes and a glass bottle.

Volume

- Volume Data for this waste stream can be found in Chapter 11.

Waste Stream Composition

- Organic debris - glass

Waste Code

- D009A (TCLP Hg) nonwastewater 


\section{LDR Treatment Standard}

- $\quad$ D009A $=$ concentration based standard $=0.20 \mathrm{mg} / \mathrm{l} \mathrm{TCLP}$; or RMERC

- D001 through D043 nonwastewaters to be land disposed must be treated to meet Universal Treatment Standards (UTS) for any underlying hazardous constituents (UHC) that may be present.

Waste Characterization

- Sample results

\section{Radiological Characterization}

- Beta/gamma emitters present

\section{SR-W063, Macroencapsulated Low-Level Waste (TC)}

This waste consists of a wide variety of miscellaneous macroencapsulated lead items contaminated with radioactive materials. The majority of the lead is encapsulated in Stainless Steel. The lead items include lead counterweighted jumpers (lead welded in pipe for balancing jumpers), cesium removal columns (CRC) (lead sandwiched between stainless steel for shielding purposes), draw-off valves, flush valves, and discarded equipment (same description as CRC configuration). These wastes generally are used as shields from radioactivity (e.g., around pipes in tank farms), as counterweights, or serve as parts of other devices. The majority of the radioactive contamination is surface contamination. The waste is generated in reactor areas, and fuel and target and separation areas, and laboratories. Future generation rates are dependent upon Site decommissioning activities. Lead that continues to serve its function is not considered a mixed waste, but is reviewed in the site's contaminated equipment program for appropriate disposition.

\section{Volume}

- Volume Data for this waste stream can be found in Chapter 11.

Waste Stream Composition

- Macroencapsulated inorganic debris

Waste Code

- D004 (Arsenic)

- D005 (Barium)

- D006A (TCLP Cd)

- D007 (Chromium)

- D008A (TCLP Pb)

- D009A (TCLP Hg)

- D010 (Selenium)

- D011 (Silver)

\section{LDR Treatment Standard}

- $\quad$ D004 $=$ concentration based standard $=5.0 \mathrm{mg} / \mathrm{l}, \mathrm{TCLP}$

- $\mathrm{D} 005=$ concentration based standard $=21 \mathrm{mg} / \mathrm{l}, \mathrm{TCLP}$

- D006A $=$ concentration based standard $=0.11 \mathrm{mg} / \mathrm{l}$, TCLP

- D007 $=$ concentration based standard $=0.60 \mathrm{mg} / \mathrm{l}$, TCLP

- $\quad$ D008A $=$ concentration based standard $=0.75 \mathrm{mg} / \mathrm{l}$, TCLP

- $\mathrm{D} 009 \mathrm{~A}=$ concentration based standard $=0.025 \mathrm{mg} / \mathrm{l}$, TCLP 
- $\quad$ D $010=$ concentration based standard $=5.7 \mathrm{mg} / \mathrm{l}$, TCLP

- D011 = concentration based standard $=0.14 \mathrm{mg} / \mathrm{l}$, TCLP

- D001 through D043 nonwastewaters to be land disposed must be treated to meet Universal Treatment Standards (UTS) for any underlying hazardous constituents (UHC) that may be present.

Waste Characterization

- Process knowledge

Radiological Characterization

- Alpha and beta/gamma emitters are present.

\section{SR-W072, Supernate or Sludge Contaminated Debris from High-Level Waste (HLW) Operations}

This waste consists of a wide variety of equipment/metal debris and other items contaminated with radioactive materials and characteristically hazardous waste. The waste is derived from contacting High Level Waste. The majority of the contamination is surface contamination. Future generation rates are dependent upon construction operations, maintenance activities, and site decommissioning activities.

Volume

- Volume Data for this waste stream can be found in Chapter 11.

Waste Stream Composition

- Inorganic debris

Waste Code

- D005 (Barium)

- D006A (TCLP Cd)

- D007 (Chromium)

- D008A (TCLP Pb)

- D009A (TCLP Hg)

- D010 (Selenium)

- D011 (Silver)

- D018 (Benzene)

LDR Treatment Standard

- $\mathrm{D} 005=$ concentration based standard $=21 \mathrm{mg} / \mathrm{l}, \mathrm{TCLP}$

- $\quad$ D006A $=$ concentration based standard $=0.11 \mathrm{mg} / \mathrm{l}$, TCLP

- $\mathrm{D} 007=$ concentration based standard $=0.60 \mathrm{mg} / \mathrm{l}$, TCLP

- $\quad$ D008A $=$ concentration based standard $=0.75 \mathrm{mg} / \mathrm{l}$, TCLP

- $\mathrm{D} 009 \mathrm{~A}=$ concentration based standard $=0.025 \mathrm{mg} / \mathrm{l}$, TCLP

- $\quad \mathrm{D} 010=$ concentration based standard $=5.7 \mathrm{mg} / \mathrm{l}$, TCLP

- D011 = concentration based standard $=0.14 \mathrm{mg} / \mathrm{l}$, TCLP

- D018 = concentration based standard $=10 \mathrm{mg} / \mathrm{kg}$

- D001 through D043 nonwastewaters to be land disposed must be treated to meet Universal Treatment Standards (UTS) for any underlying hazardous constituents (UHC) that may be present. 


\section{Waste Characterization}

- Process knowledge

\section{Radiological Characterization}

- Alpha and beta/gamma emitters are present SR-W078, LDR Hazardous Waste Awaiting Radiological Screening

This stream has been closed, and all previous 078 wastes have been screened. The waste stream is composed of dark liquids, thick organic liquids, and heterogeneous solids generated site-wide in areas where radiological contamination is possible but uncertain. The physical makeup of the remainder of this waste stream has prevented adequate radiological characterization to date because the waste is either heterogeneous, requiring development of special, recognized sampling protocols to satisfactorily sample the waste for characterization; or, is opaque, requiring specialized analytical methods to quantify and qualify radiological waste constituents.

Volume

- Volume data for this waste stream can be found in Chapter 11.

Waste Stream Composition

- Heterogeneous solids, dark liquids, and thick organic liquids

Waste Codes

- D001A (ignitable high TOC)

- D004 (TCLP As)

- D005 (TCLP Ba)

- D006A (TCLP Cd)

- D007 (TCLP Cr)

- D008A (TCLP Pb)

- D009A (TCLP Hg)

- D010 (TCLP Se)

- D011 (TCLP Ag)

- D012 (Endrin)

- D013 (Lindane)

- D014 (Methoxychlor)

- D015 (Toxaphene)

- D016 (2,4-D)

- D017 (2, 4, 5-TP [Silvex])

- D018 (Benzene)

- D020 (Chlordane)

- D035 (Methylethyl ketone)
- D040 (Trichloroethylene)

- F001 (spent halogenated degreasing solvents)

- F002 (spent halogenated solvents)

- F003 (spent nonhalogenated solvents)

- F005 (spent nonhalogenated solvents)

- F027 (Dioxin-containing waste)

- P051 (Endrin)

- P123 (Toxaphene)

- U045 (Methyl chloride)

- U061 (DDT)

- U108 (1,4-Dioxane)

- U129 (Lindane)

- U210 (Tetrachloroethylene)

- U226 (1,1,1 trichloroethane)

- U228 (Trichloroethylene)

- U247 (Methoxychlor)

- Nonwastewater

LDR Treatment Standards

- D001 = specified technology $=$ RORGS or CMBST

- D004 $=$ concentration based standard $=5.0 \mathrm{mg} / \mathrm{l} \mathrm{TCLP}$

- D005 $=$ concentration based standard $=21 \mathrm{mg} / \mathrm{l} \mathrm{TCLP}$

- $\mathrm{D} 006=$ concentration based standard $=0.11 \mathrm{mg} / \mathrm{l}$ TCLP

- $\mathrm{D} 007=$ concentration based standard $=0.60 \mathrm{mg} / \mathrm{l}$ TCLP

- $\mathrm{D} 008=$ concentration based standard $=0.75 \mathrm{mg} / \mathrm{l} \mathrm{TCLP}$

- D009 $=$ concentration based standard $=0.025 \mathrm{mg} / \mathrm{l}$ TCLP

- D011 = concentration based standard $=0.14 \mathrm{mg} / \mathrm{l}$ TCLP

- D012 = concentration based standard $=0.13 \mathrm{mg} / \mathrm{kg}$

- $\mathrm{D} 013=$ concentration based standard $=0.066 \mathrm{mg} / \mathrm{kg}$

- $\mathrm{D} 014=$ concentration based standard $=0.18 \mathrm{mg} / \mathrm{kg}$ 
- $\mathrm{D} 015=$ concentration based standard $=2.6 \mathrm{mg} / \mathrm{kg}$

- D016 = concentration based standard $=10 \mathrm{mg} / \mathrm{kg}$

- $\mathrm{D} 017=$ concentration based standard $=7.9 \mathrm{mg} / \mathrm{kg}$

- $\mathrm{D} 018=$ concentration based standard $=10 \mathrm{mg} / \mathrm{kg}$

- $\mathrm{D} 020=$ concentration based standard $=0.26 \mathrm{mg} / \mathrm{kg}$

- D035 $=$ concentration based standard $=36 \mathrm{mg} / \mathrm{kg}$

- $\mathrm{D} 040=$ concentration based standard $=6.0 \mathrm{mg} / \mathrm{kg}$

- F001 \& F002 = concentration based standard $=6.0-30 \mathrm{mg} / \mathrm{kg}$

- $\mathrm{F} 003=$ concentration based standard $=0.75 \mathrm{mg} / \mathrm{l}, \mathrm{TCLP}-160 \mathrm{mg} / \mathrm{kg}$

- $\mathrm{F} 005=$ concentration based standard $=4.8 \mathrm{mg} / \mathrm{l} \mathrm{TCLP}-170 \mathrm{mg} / \mathrm{kg}$, except 2-Ethoxyethanol, 2-Nitropropane $=$ CMBST

- F027 = concentration based standard $=10-28 \mathrm{mg} / \mathrm{kg}$ (depends on specific chemical)

- $\mathrm{P} 051=$ concentration based standard $=0.13 \mathrm{mg} / \mathrm{kg}$

- $\mathrm{P} 123=$ concentration based standard $=2.6 \mathrm{mg} / \mathrm{kg}$

- $\mathrm{U} 045=$ concentration based standard $=30 \mathrm{mg} / \mathrm{kg}$

- $\mathrm{U} 061=$ concentration based standard $=0.087 \mathrm{mg} / \mathrm{kg}$

- $\mathrm{U} 108=$ specified technology $=$ CMBST; or $=$ concentration based standard $=170 \mathrm{mg} / \mathrm{kg}$

- $\mathrm{U} 129=$ concentration based standard $=0.066 \mathrm{mg} / \mathrm{kg}$

- $\mathrm{U} 210=$ concentration based standard $=6.0 \mathrm{mg} / \mathrm{kg}$

- $\mathrm{U} 226=$ concentration based standard $=6.0 \mathrm{mg} / \mathrm{kg}$

- $\mathrm{U} 228=$ concentration based standard $=6.0 \mathrm{mg} / \mathrm{kg}$

- $\mathrm{U} 247=$ concentration based standard $=0.18 \mathrm{mg} / \mathrm{kg}$

- D001 through D043 nonwastewaters to be land disposed must be treated to meet Universal Treatment Standards (UTS) for any underlying hazardous constituents (UHC) that may be present.

\section{Waste Characterization}

- Process knowledge and sampling and analysis were used to characterize the waste stream.

- Confidence level is high because there is extensive process knowledge and because sampling and analysis has been performed on portions of the waste stream.

\section{Radiological Characterization}

- Unknown at this time. Awaiting characterization. Level of radiological contamination very low, if present. 
THIS PAGE INTENTIONALLY LEFT BLANK. 


\section{Chapter 4. Mixed Transuranic Waste (MTRU)}

The current DOE strategy for management of MTRU waste is to maintain the MTRU wastes in safe interim storage; to characterize, certify, process if necessary, and package the wastes to meet the Waste Acceptance Criteria (WAC) of the Waste Isolation Pilot Plant (WIPP); and to permanently dispose of applicable MTRU waste in WIPP. The Defense Authorization Bill for federal FY 97, which contained amendments to the 1992 WIPP Land Withdrawal Act, was signed by the President on September 22, 1996, and the Secretary of Energy declared WIPP open and ready to receive waste in May 1998.

The amendments also exempt DOE from the requirement to obtain a RCRA no-migration determination from EPA. EPA agrees that the no-migration determination is redundant to the more stringent radioactive waste disposal standard, and that the exemption will not jeopardize the environment. WIPP has obtained a RCRA Part B Permit from the State of New Mexico to receive mixed TRU waste for disposal.

Site-specific information is included in the following section to outline activities being performed at the Savannah River Site to maintain safe compliant storage, waste characterization activities, and other activities planned to support the ultimate goal of shipment to and disposal at WIPP.

This chapter reviews the project activity schedules that have been planned for the treatment of MTRU waste in accordance with Section 2.2 of this volume. Section 4.1 discusses the site's MTRU waste management approach and section 4.2 details the MTRU waste streams that are proposed to be shipped to WIPP for treatment. Refer to Table 1.1, Chapter 1, of Volume II of the STP for the user's guide. The user's guide identifies each mixed waste stream, the preferred treatment option (PO), and the location where the waste stream is discussed in Volumes I and II of the STP.

\subsection{Site MTRU Waste Management Approach}

TRU waste is defined in DOE Order 435.1 as waste contaminated with alpha-emitting transuranic radionuclides (radionuclides with atomic numbers greater than 92) with half-lives greater than twenty (20) years and radionuclide concentrations greater than 100 nanocuries per gram (nCi/g). TRU waste at SRS that includes hazardous constituents as identified in 40 CFR 261 and SCHWMR R61-79.261 is managed in accordance with DOE orders and SCHWMR, and is referred to as mixed TRU waste (MTRU).

MTRU waste streams are, and have been, generated primarily by Plutonium Separations Facilities and the Analytical Laboratories. Other past generators of significantly smaller volumes include Naval Fuels Facility, the Reactor Facility, the Fuel Fabrication Facility, the High-Level Waste Tank Farms, and the Solid Waste Management Facility. In the 1970's, SRS received a large volume of MTRU waste from offsite generators including the Los Alamos National Laboratory, Knolls Atomic Power Laboratory, and the DOE Mound Site. MTRU waste shipments have been received from the DOE Mound Site since September 2001 (see Vol. II, Sec. 4.2.2).

MTRU waste generated at SRS is primarily job control waste, which includes combinations of the following: plastic, paper, rubber, glassware, metal items, lead-lined gloves, filters, used equipment, and other contaminated materials from routine operations.

Due to the variety of container types, MTRU waste is generally described by its container: drums, polyethylene boxes, concrete casks, large steel black boxes, and other odd-sized containers. The type of container also dictates the storage configuration, for example, drums are either stored in culverts, which are stored on uncovered pads exposed to the weather or stored directly on covered pads.

Currently, five waste streams are managed as MTRU waste. 
The waste streams identified as Mixed TRU waste are:

\begin{tabular}{lll}
$\begin{array}{c}\text { Waste Stream } \\
\text { No. }\end{array}$ & & \multicolumn{1}{c}{ Description } \\
\cline { 1 - 1 } SR-W006 & & CH Mixed TRU/Liquids \\
SR-W026 & & CH Mixed TRU/Thirds \\
SR-W027 & & CH Mixed TRU/F-Listed Solvents \\
SR-W053 & & Rocky Flats Ash* \\
SR-W089 & & MTRU Waste Received From Mound Site
\end{tabular}

\section{SRS Solid Waste Management Strategy}

The SRS solid waste management strategy supports and is in alignment with the National MTRU Program Initiatives. The MTRU waste program is shipping to WIPP, MTRU waste operations at SRS are characterizing containers for certification and packaging for shipping to WIPP for final disposal. WIPP opened for acceptance of the DOE-Complex MTRU waste December 1999, and SRS made seven (7) shipments of TRU waste in FY01, 16 in FY02, 185 in FY03, and 239 in FY04.

\section{Plan Assumptions}

The MTRU waste plan is based on the following key assumptions:

- Funding is available to support the plan.

- Negotiations with WIPP, the Nuclear Regulatory Commission (NRC), and other environmental agencies to revise the restrictive transportation limits of the TRUPACT II are successful.

- WIPP does not close prior to 2034.

- Processes are planned to be put in place to process MTRU waste for shipment to WIPP.

- Assay techniques for $\mathrm{Pu}^{238}$ contaminated wastes and larger containers will be pursued and implemented at SRS using onsite and offsite technology to meet the requirement of the WIPP WAC and WIPP Quality Assurance Program.

- A Modular TRU Waste Repackaging System is planned to be operational at SRS from 2005-2007 to process MTRU waste drums for shipment to WIPP.

\section{Plan Activities}

The SRS solid waste management strategy addresses the following activities and provides a path forward for resolution:

- Interim storage

- TRU Waste Certification

- TRU Waste Processing

- Outyear Schedule

\section{Interim Storage}

SRS continues to safely store MTRU waste containers in preparation for WIPP characterization and disposal. Operations commenced in 2003 to vent approximately 4000 drums that were generated prior to 1986 . Vents are installed to allow the release of potential hydrogen gas build-up.

\section{MTRU Waste Certification/Characterization}

Activities in the MTRU waste area involve extensive preparation for the certification of containers to meet the WIPP waste acceptance criteria. The WIPP certification program requires characterization of the waste matrix through radionuclide assay, radiography, and headspace gas sampling for all waste containers. In addition, a 
representative number of containers must be opened for visual examination to confirm the characterization information. SRS has designated a portion of TRU pad 6 for the visual examination (VE) facility.

SRS utilizes a Mobile Vendor for the inspection/characterization and shipment of TRU waste from SRS to WIPP. The Mobile Vendor mobilized in 2QFY01 and began characterization operations in 4QFY01. The Mobile Vendor is expected to process and ship waste from SRS to WIPP through FY06. The Mobile Vendor provides this characterization and shipping capability through the use of four mobile systems. The first is a real time radiography trailer that is used to X-ray TRU drums to determine drum contents and waste attributes. Following x-ray the drums are processed through one of two non-destructive assay trailers. One system uses an integrated gamma and imaging passive/active neutron/gamma energy analysis (IPAN/GEA) mobile waste assay trailer. The IPAN/GEA provides information to determine the isotopic composition of each TRU waste drum. A second system uses gamma spectroscopy (IQ3). After assay the drums are placed into heated sealand storage containers for 72 hours prior to head space gas sampling. The fourth mobile system is the drum headspace gas sampling system. This system utilizes a gas chromatograph (GC) and mass spectrometer (MS) to analyze the constituents of the TRU drum headspace gases. The drums are then moved to the final system which is the mobile loading unit. This system contains the adjustable center gravity lift fixture, shrink wrap machine and equipment necessary to configure and load TRU drums into TRUPACT-II containers for transport to WIPP for disposal. These systems are currently set-up on existing TRU waste storage Pads \#4 and \#3, although future systems, such as additional radiography trailers, may be set up near the TRU pads. In these situations, containers are transported one at a time for appropriate screening or analysis and returned to the TRU pads. This process is supported by existing SRS infrastructure to include the Visual Examination Facility currently on TRU Pad \#6.

\section{MTRU Waste Processing}

The Modular Repackaging Facility, currently on Pad 19, is a hazard category II facility that will sort and segregate TRU waste contained in drums. It will remove prohibited items and will repackage waste to meet transportation limits for shipment to WIPP for disposal.

For large containers, a hazard category II type process will be implemented to address solids, liquids, sludges, and soil wastes contaminated with alpha-emitting transuranic radionuclides for disposal. This includes, at a minimum, repackaging, sorting, size reduction, and disposal at WIPP. This process uses equipment that has been demonstrated on similar activities or the technology development is in progress to support the facility's equipment needs.

\subsection{MTRU Waste Streams Proposed for Shipment to WIPP}

MTRU waste is divided into waste groupings based primarily on the containers that hold the waste. The amount of detailed information on the contents of each waste container is limited. The container type, generator, and radionuclide data provide information that allows the inventory of MTRU waste to be placed into waste groupings that support planning for future processing to meet the requirement of the WIPP WAC. This section discusses the waste streams that are proposed to be shipped to WIPP for disposal. Refer to Table 1.3, Chapter 1, Volume II for EPA Hazardous Waste Code Subcategories. 


\subsubsection{MTRU Waste Requiring Characterization/Certification for WIPP}

This section describes MTRU waste streams, which require characterization and certification prior to acceptance for disposal at WIPP. The section is divided into two subsections for waste stream categories as follows:

\subsubsection{Defense-Related TRU Job Control Waste Group}

\subsubsection{Defense-Related TRU Job Control Waste Group}

The preferred option for this waste group is to assay, sort, process, and characterize the waste material for drummed waste or processes to be developed for non-drummed waste in existing facilities (CAT II and CAT III), followed by preparation for shipment and disposal at WIPP.

Waste streams within this waste group include the following:

SR-W026, CH Mixed TRU /Thirds

SR-W027, CH Mixed TRU /F-Listed Solvents

\section{General Information}

\section{SR-W026, CH Mixed TRU /Thirds}

This waste stream is a job control waste and is composed primarily of organic solids such as booties, lab coats, floor sweepings, rags, labware, and other job control waste generated primarily through separation activities. A small percentage $(<5 \%)$ of this waste stream includes sludges, resins, filters, and miscellaneous waste. The sludges are caustic evaporated residues that have been neutralized and absorbed.

Volume

- Volume data for this waste stream can be found in Chapter 11.

Waste Stream Composition

- Organic debris

Waste Code

- D001C (Low TOC Ignitable)

- P048 (2, 4-Dinitrophenol)

- D003D (Water Reactive)

- D004 (TCLP As)

- D006A (TCLP Cd)

- D007 (TCLP Cr)

- D008A (TCLP Pb)

- D009A (TCLP Hg)

- D011 (TCLP Ag)

- D018 (Benzene)

- D019 (Carbon tetrachloride)

- D022 (Chloroform)

- D023 (o-Cresol)

- D024 (m-Cresol)

- P113 (Thallic oxide)

- P120 (Vanadium pentoxide)

- U002 (Acetone)

- U032 (Calcium chromate)

- U052 (Creosols-mixed)

- U080 (Methylene chloride)

- U133 (Hydrazine)

- U134 (Hydrogen fluoride)

- U144 (Lead acetate)

- U151C (Low Mercury)

- U154 (Methanol)

- D025 (p-Cresol)

- D026 (Cresols)

- P012 (Arsenic trioxide)

- P015 (Beryllium powder)

- U161 (Methyl isobutyl ketone)

- $\mathrm{U} 209$ (1, 1, 2, 2-Tetrachloroethane)

- U211 (Carbon tetrachloride)

- U220 (Toluene)

- Nonwastewater

- $\mathrm{U} 226$ (1, 1, 1-Trichloroethane)

- U239 (Xylenes) 
- Manage at the WIPP.

The preferred option is to process the waste in the processing facilities such as the Modular TRU Waste Repackaging Facility to sort and segregate and other waste handling technologies to characterize, repackage, and certify the waste to meet TRUPACT shipping requirements for disposal at WIPP.

Processing will include removing non-compliant items found from the waste. This option is preferred because it has the lowest cost and has the lowest risk for delays due to permitting, technical barriers, and system implementability. It uses equipment that has been demonstrated on similar activities or the technology development is in progress to support the facilities equipment needs.

\section{Waste Characterization}

- Sampling and analysis will be used to characterize the waste stream (NDE, NDA, and headspace sampling).

- Confidence level is medium based on the varying composition of the job waste and the exact contents of specific containers.

\section{Radiological Characterization}

- Total activity is $>100 \mathrm{nCi} / \mathrm{g}$.

- Beta/gamma emitters $\left(\mathrm{H}^{3}, \mathrm{Co}^{60}\right.$, and $\left.\mathrm{Cs}^{137}\right)$ are present.

- Alpha emitters $\left(\mathrm{Pu}^{238}, \mathrm{Pu}^{239}, \mathrm{Pu}^{240}, \mathrm{Pu}^{241}, \mathrm{Am}^{241}\right.$, and $\left.\mathrm{Cm}^{244}\right)$ are present.

- Waste is primarily contact handled with a small volume of remote handled $(<200 \mathrm{mR} / \mathrm{hr})$.

\section{SR-W027, CH Mixed TRU /F-Listed Solvents}

This waste stream is a job control waste composed primarily of solids such as booties, lab coats, floor sweepings, rags, labware, and other job control waste generated primarily from separation activities for plutonium production. This waste differs from SR-W026 because solvent rags are suspected to be present. A conservative interpretation of the mixture rule causes contents of containers to be characterized with listed solvent waste codes due to the presence of solvent rags.

\section{Volume}

- Volume data for this waste stream can be found in Chapter 11.

Waste Stream Composition

- Organic debris

Waste Codes

- D001C (Low TOC Ignitable)

- P012 (Arsenic trioxide)

- D003D (Water Reactive)

- D004 (TCLP As)

- D006A (TCLP Cd)

- D007 (TCLP Cr)

- D008A (TCLP Pb)

- D009A (TCLP Hg)

- D011 (TCLP Ag)

- D018 (Benzene)

- D019 (Carbon tetrachloride)

- P015 (Beryllium dust)

- P048 (2, 4-Dinitrophenol)

- P113 (Thallic oxide)

- P120 (Vanadium pentoxide)

- U002 (Acetone)

- U032 (Calcium chromate)

- U052 (Cresols-mixed)

- U080 (Methylene chloride)

- U133 (Hydrazine)

- D022 (Chloroform)

- D023 (o-Cresol)

- D024 (m-Cresol)

- D025 (p-Cresol)

- D026 (Cresol)

- U134 (Hydrogen fluoride)

- U144 (Lead acetate)

- U151C (Low Mercury)

- U154 (Methanol)

- U161 (Methyl isobutyl ketone) 
- F001 (Spent halogenated degreasing solvents)

- F002 (Spent halogenated solvents)

- F003 (Spent nonhalogenated solvents)

- F005A (Spent nonhalogenated solvents)

- Nonwastewater

\section{LDR Treatment Standard}

- Manage at the WIPP.

The preferred option is to process this waste in the processing facilities such as the Modular TRU Waste Repackaging Facility to sort and segregate and other waste handling technologies to characterize, repackage and certify waste to meet the TRUPACT shipping requirements for disposal at WIPP.

Processing will include removing non-compliant items found from the waste. This option is preferred because it has the lowest cost and has the lowest risk for delays due to permitting, technical barriers, and system implementability. It uses equipment that has been demonstrated on similar activities or the technology development is in progress to support the facilities equipment needs.

\section{Waste Characterization}

- Sampling and analysis are used to characterize the waste stream.

- Confidence level is medium based on the varying composition of the job waste and the exact contents of specific waste containers.

\section{Radiological Characterization}

- Total activity is $>100 \mathrm{nCi} / \mathrm{g}$.

- Beta/gamma emitters $\left(\mathrm{H}^{3}, \mathrm{Co}^{60}\right.$, and $\left.\mathrm{Cs}^{137}\right)$ are present.

- Alpha emitters $\left(\mathrm{Pu}^{238}, \mathrm{Pu}^{239}, \mathrm{Pu}^{240}, \mathrm{Pu}^{241}, \mathrm{Am}^{241}\right.$, and $\left.\mathrm{Cm}^{244}\right)$ are present.

- Waste is primarily contact handled $(<200 \mathrm{mR} / \mathrm{hr})$ with a small volume of remote handled.

\subsubsection{Reserved.}

\subsubsection{Other MTRU Waste Streams for Shipment to WIPP}

The preferred option for this waste group is to characterize the waste material followed by preparation for shipment and disposal.

Waste streams within this waste group include the following:

SR-W006, CH Mixed TRU/Liquids

SR-W053, Rocky Flats Ash

SR-W089, CH Mixed TRU from Mound for Shipment to WIPP

\section{General Information}

\section{SR-W006, CH Mixed TRU/Liquids}

This waste stream is defense-related MTRU waste, consisting of a liquid waste generated from plutonium extraction analytical procedures at the Savannah River National Laboratory (SRNL). It consists of a homogeneous, xylene based, liquid chelating agent. This is a small volume waste stream and is currently stored in compliance with RCRA in a satellite accumulation area at SRNL.

Volume 
- Volume data for this waste stream can be found in Chapter 11.

Waste Stream Composition

- Organic liquid

Waste Code

- D001A (Ignitable-High TOC)

- Nonwastewater

LDR Treatment Standard

- Manage at the WIPP

Waste Characterization

- Sampling and analysis are used to characterize the waste stream.

- Confidence level is high based upon knowledge of the chemicals used in the analytical procedures.

\section{Radiological Characterization}

- Total activity is $>100 \mathrm{nCi} / \mathrm{g}$.

- Alpha emitters $\left(\mathrm{Pu}^{238}, \mathrm{Pu}^{239}, \mathrm{Pu}^{240}, \mathrm{Pu}^{241}, \mathrm{Pu}^{242}\right.$, and $\left.\mathrm{Am}^{241}\right)$ are present.

- Waste is contact handled $(<200 \mathrm{mR} / \mathrm{hr})$.

\section{SR-W053, Rocky Flats Ash}

This waste consists of a small volume of ash sent from Rocky Flats to SRS for research into plutonium recovery. Courts in the State of Colorado declared Rocky Flats' ash hazardous based on chemical analysis of Flisted solvent waste processed in the Rocky Flats incinerator. Upon learning of the Colorado court action, SRS placed the ash in a RCRA satellite accumulation area. SRS recently completed a treatability study with this material at SRNL.

Volume

- Volume data for this waste stream can be found in Chapter 11.

Waste Stream Matrix

- Inorganic sludge/particulate

Waste Codes

- D004 (TCLP As)

- D005 (TCLP Ba)

- D006A (TCLP Cd)

- D007 (TCLP Cr)

- D008A (TCLP Pb)

- D009A (TCLP Hg)

- D010 (TCLP Se)

- F001 (Spent halogenated degreasing solvents)

- F002 (Spent halogenated solvents)

- F005A (Spent nonhalogenated solvents)

LDR Treatment Standard

- Manage at the WIPP 


\section{Waste Characterization}

- Process knowledge is used to characterize the waste stream.

- Confidence level is low. No analytical data is available, and the material is from another DOE site.

- This ash was declared mixed waste after SRS had the material in a vault and was handling the waste as a Special Nuclear Material (SNM).

\section{Radiological Characterization}

- Transuranic-alpha emitters $\left(\mathrm{Am}^{241}, \mathrm{Pu}^{238}, \mathrm{Pu}^{239}, \mathrm{Pu}^{240}, \mathrm{Pu}^{241}\right.$, and $\left.\mathrm{Pu}^{242}\right)$ are present.

- Waste is contact handled $(<200 \mathrm{mR} / \mathrm{hr})$.

\section{SR-W089, CH Mixed TRU from Mound for Shipment to WIPP}

This waste stream is a defense-related job control and D\&D waste and is composed of organic solids such as booties, lab coats, floor sweepings, rags, labware, soils, and other job control waste along with gloveboxes, fume hoods, and general D\&D debris. A small percentage $(<5 \%)$ of this waste stream includes sludges, resins, filters, and miscellaneous waste. The sludges will be neutralized and solidified.

\section{Volume}

- Volume data for this waste stream can be found in Chapter 11.

Waste Stream Composition

- Organic debris

Waste Code

- D001C (Low TOC Ignitable)

- D004 (TCLP As)

- D006A (TCLP Cd)

- D007 (TCLP Cr)

- D008A (TCLP Pb)

- D009A (TCLP Hg)

- D011 (TCLP Ag)

- Nonwastewater

- Manage at the WIPP.

The preferred option is to process the waste to sort and segregate and use other waste handling technologies to size-reduce, characterize, repackage, and certify the waste to meet TRUPACT shipping requirements for disposal at WIPP.

Processing will include removing non-compliant items found from the waste. Treatment will remove, reduce, or destroy to meet LDR requirements to comply with the WIPP RCRA Part B permit. This option is preferred because it has the lowest cost and has the lowest risk for delays due to permitting, technical barriers, and system implementability. It uses equipment that has been demonstrated on similar activities or the technology development is in progress to support the facilities equipment needs.

\section{Waste Characterization}

- Sampling and analysis will be used to characterize the waste stream (NDE, NDA, and headspace sampling).

- Confidence level for the legacy waste is medium based on historical knowledge and the implementation of the Real Time Radiography.

- Confidence level for the D\&D waste is high based on the implementation of the Mound TRU Certification process and oversight by SRS personnel. 
Radiological Characterization

- Total activity is $>100 \mathrm{nCi} / \mathrm{g}$.

- Beta/gamma emitters $\left(\mathrm{H}^{3}, \mathrm{Co}^{60}\right.$, and $\left.\mathrm{Cs}^{137}\right)$ are present.

- Alpha emitters $\left(\mathrm{Pu}^{238}, \mathrm{Pu}^{239}, \mathrm{Pu}^{240}, \mathrm{Pu}^{241}, \mathrm{Am}^{241}\right.$, and $\left.\mathrm{Cm}^{244}\right)$ are present.

- Waste is exclusively contact handled $(<200 \mathrm{mR} / \mathrm{hr})$. 
THIS PAGE INTENTIONALLY LEFT BLANK. 


\section{Chapter 5. High-Level Radioactive Waste (HLW)}

The following waste streams are in the mixed high-level waste category. This chapter discusses the high-level waste streams and the available treatment. Refer to Table 1.1, Chapter 1, Volume II of the STP for the user's guide. The user's guide identifies each mixed waste stream, the preferred treatment option (PO), and the location where the waste stream is discussed in Volumes I and II of the STP. Also refer to Table 1.3, Chapter 1, Volume II for EPA Hazardous Waste Code Subcategories.

\section{Mixed High-Level Waste Group for Vitrification}

Vitrification in the DWPF is the preferred treatment option for certain mixed waste streams, including, but not limited to, the following:

SR-W016, 221-F Canyon High-Level Liquid Waste

SR-W017, 221-H Canyon High Level Liquid Waste

\section{General Information}

\section{SR-W016, 221-F Canyon High-Level Liquid Waste}

This waste is an aqueous liquid containing fission products generated from deactivation of the 221-F Canyon facility. The extraction of plutonium from reactor targets assemblies and dissolution of spent fuel rods was suspended in FY02

Volume

- Volume data for this waste stream can be found in Chapter 11.

Waste Stream Composition

- Aqueous liquid

Waste Code

- D002 (corrosive waste managed as wastewater)

- D005 (TCLP Ba)

- D007 (TCLP Cr)

- D008A (TCLP Pb)

- D009A (TCLP Hg)

- D011 (TCLP Ag)

- Nonwastewater slurry

LDR Treatment Standard

- All waste codes $=$ specified technology $=$ HLVIT

\section{Waste Characterization}

- Sampling and analysis are used to characterize the waste stream.

- Confidence level is high based on availability of analysis, with the exceptions of TCLP.

\section{Radiological Characterization}

- Total activity for radiological characterization is $\sim 10.5 \mathrm{Ci} / \mathrm{gal}$.

- Alpha emitters $\left(\mathrm{U}^{235}, \mathrm{U}^{238}, \mathrm{Pu}^{238}, \mathrm{Pu}^{239}, \mathrm{Pu}^{240}, \mathrm{Pu}^{241}, \mathrm{Am}^{241}, \mathrm{Cm}^{241}\right.$, and others) are present. 
- Beta/gamma emitters $\left(\mathrm{Sr}^{90}, \mathrm{Ru}^{106}, \mathrm{Zr}^{95}, \mathrm{Nb}^{95}, \mathrm{Rh}^{106}, \mathrm{Cs}^{137}, \mathrm{Ce}^{144}, \mathrm{Pr}^{144}, \mathrm{Pm}^{147}, \mathrm{H}^{3}\right.$, and others) are present.

- Waste is remote handled.

- High-level waste

\section{SR-W017, 221-H Canyon High-Level Liquid Waste}

This waste stream is an aqueous liquid containing mixed fission products from the H-Canyon facility in support of the modified PUREX process. The stream also contains decontamination solution from maintenance activities in the H-Area High-Level Waste Tank Farm. H-Canyon waste materials are generated from the recovery of enriched uranium from fuel tubes.

Volume

- Volume data for this waste stream can be found in Chapter 11.

Waste Stream Composition

- Aqueous liquid

Waste Code

- D002A (corrosive waste managed as wastewater)

- D005 (TCLP Ba)

- D007 (TCLP Cr)

- D008A (TCLP Pb)

- D009A (TCLP Hg)

- D011 (TCLP Ag)

- Nonwastewater slurry

LDR Treatment Standard

- All waste codes = specified technology = HLVIT

\section{Waste Characterization}

- Sampling and analysis are used to characterize the waste stream.

- Confidence level is high based on availability of analysis, with the exceptions of TCLP.

\section{Radiological Characterization}

- Total activity for radiological characterization is $\sim 12 \mathrm{Ci} / \mathrm{gal}$.

- Alpha emitters $\left(\mathrm{U}^{235}, \mathrm{U}^{238}, \mathrm{Pu}^{238}, \mathrm{Pu}^{239}, \mathrm{Pu}^{240}, \mathrm{Pu}^{241}, \mathrm{Am}^{241}\right.$, and $\left.\mathrm{Cm}^{241}\right)$ are present.

- Beta/gamma emitters $\left(\mathrm{Sr}^{90}, \mathrm{Ru}^{106}, \mathrm{Zr}^{95}, \mathrm{Nb}^{95}, \mathrm{Rh}^{106}, \mathrm{Cs}^{137}, \mathrm{Ce}^{144}, \mathrm{Pr}^{144}, \mathrm{Pm}^{147}\right.$, and $\left.\mathrm{H}^{3}\right)$ are present.

- Waste is remote handled.

- High-level waste

The following information applies to all the waste streams in this waste group.

\section{Technology and Capacity Needs}

Vitrification is the specified technology for all of the waste codes in SRS high-level wastes. These wastes are generated from the extraction of plutonium and the recovery of enriched uranium. DWPF is designed with capacity to treat the identified, existing, and future high-level liquid waste streams at SRS.

The high-level waste tanks in F Area and H Area currently store a total volume of approximately 137,900 (as of September 30, 2004) of salt solution, saltcake or precipitate, and sludge generated mostly from dissolution of target assemblies irradiated in the SRS reactors. It is expected that an additional volume of high- 
level liquid waste from both F Canyon and H Canyon will be generated at SRS in the next three years. The treatment schedule prioritizes the removal of waste from tanks that are at most risk. These are the single-walled tanks, and tanks that have only a partial secondary containment structure.

\section{Treatment Option Information}

A general schematic diagram of the high-level waste treatment process at SRS is shown below.

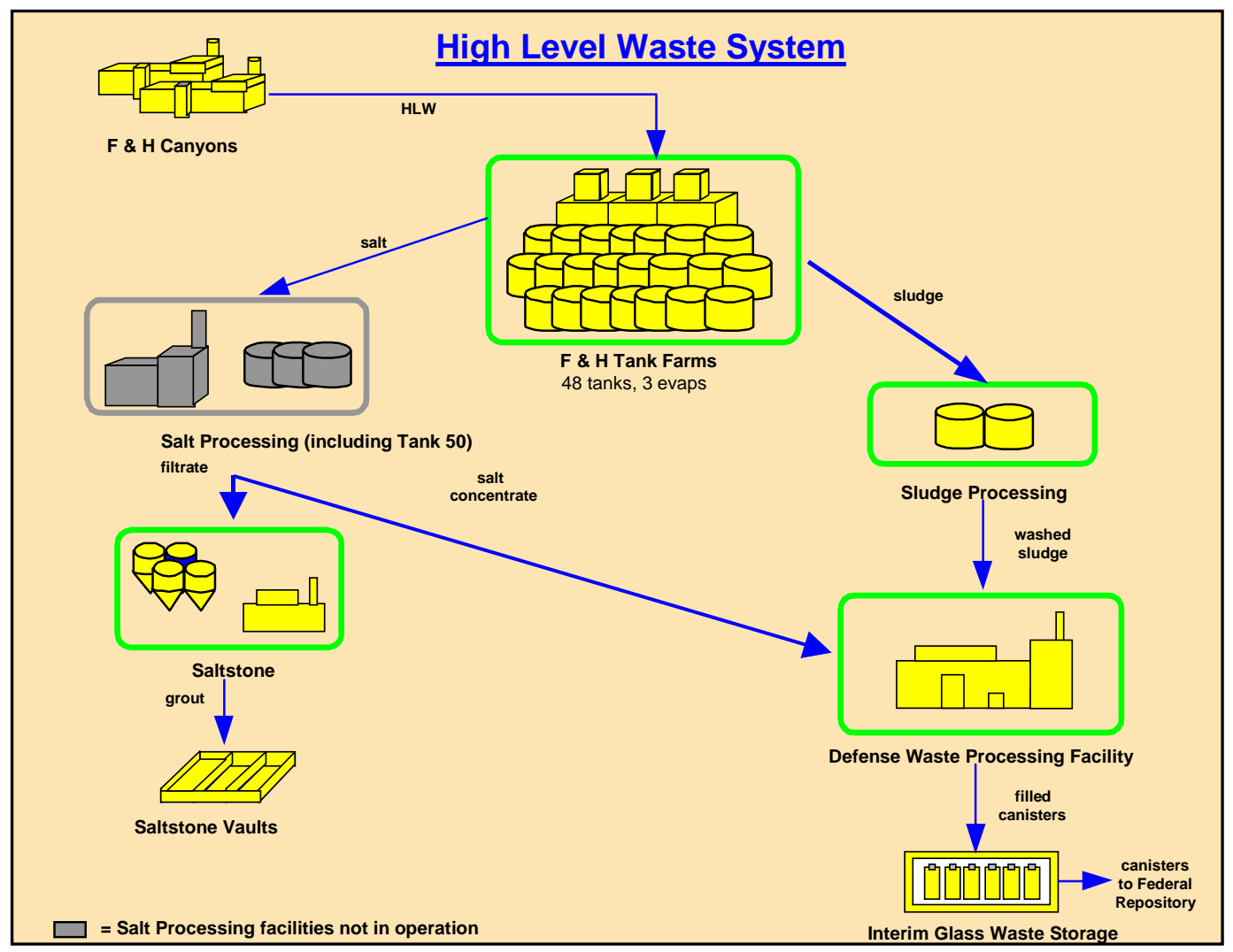

The F- and H- Area HLW Tank Farms contain waste tanks and evaporator systems to manage and treat the high-level radioactive wastewaters generated by SRS operations. These units function to receive fresh wastes, allow radioactive decay by waste aging, provide preliminary clarification by gravity settling, and concentrate dissolved salts by evaporation. The low activity aqueous portion (overheads from the evaporator systems) is transferred to ETP for final treatment prior to discharge to the Upper Three Runs Creek.

The H-Area HLW Tank Farm also contains process units to treat the accumulated sludges and salts. The sludge processing operation is designed to prepare the sludges for transfer to the DWPF Vitrification Facility for further treatment.

Once Salt Waste is acceptable for On Site Disposal it is sent to the Saltstone Manufacturing Facility and is solidified in vaults in Z-Area.

Vitrification has been identified by EPA as the specified technology for treatment of high-level waste. Borosilicate glass has been determined to be the best stabilization matrix.

TCLP tests of simulated high-level wastes were done on both expected metal levels of wastes to be processed in DWPF and at three times the level of metal expected. These tests indicated that the waste form produced at 
DWPF will be below the hazardous waste characteristic limits for toxicity (reference WSRC-IM-91-116-13, $\operatorname{Rev} 0)$.

\section{Facility Status}

On March 7, 1996, DWPF commenced operation by transferring mixed waste "sludge" from the H-Area Tank Farm to the vitrification facility. During 1996, shake-down operations occurred utilizing batch processes of diluted and full-strength mixed waste sludge until conditions of typical, routine operation had been achieved. Currently, DWPF is in "sludge only" operation. Precipitate or "salt" operations are not expected to commence until after a salt processing technology is demonstrated.

\section{Regulatory Status}

DWPF is operated under an industrial wastewater treatment facility permit. Several permit modifications have been issued since the DWPF was first designed for new construction to remove interfering contaminants or to make the operation safer.

\section{Treatment Option Status and Uncertainties}

As of commencement of operations, DWPF continues to vitrify high-level radioactive waste. The waste is then poured into stainless steel canisters, which are placed in interim storage at SRS awaiting final disposition at a Federal Repository.

\section{Budget Status}

A budget evaluation was completed for the treatment of high-level liquid waste streams. The evaluation appeared in a document titled High-Level Waste System Plan, Revision 13, March 2002 (HLW-2002-00025) and in the Performance Management Plan Supplement to the HLW System Plan Rev. 13 (the "PMP"). Information in these documents allows SRS to evaluate funding scenarios needed to ensure:

1. Safe storage of high level waste

2. Risk reduction progress by removing waste from high risk tanks

3. Waste immobilization by operating DWPF

4. Selection, design, construction, and startup of the salt processing facility

In addition, funding levels were developed so that regulatory commitments, as defined in the $F / H$ Area High-Level Waste Removal Plan and Schedule (WSRC-RP-93-1477, Rev. 0), submitted to the regulators November 9, 1993, can be met. This schedule was revised by the High-Level Waste (HLW) Removal Schedule, Revision 1 which was approved in 2002 by the regulators (EPA and SCDHEC). By letter of August 26, 2004 (DC-04-0155), DOE has requested approval from EPA-IV and SCDHEC of revised regulatory commitment dates for the operational closure of Tanks $19 \mathrm{~F}$ and $18 \mathrm{~F}$.

Under these conditions, the high-level waste treatment program for the F- and H-Canyon high-level liquid wastes can be completed by 2028 .

\section{Uncertainty Issues}

Salt Processing was suspended in January 1998 at the In-Tank Precipitation (ITP) Facility because the facility could not cost effectively meet both the safety and production requirements for the High Level Waste System. In October 2001, the DOE approved a Record of Decision (ROD) for the Savannah River Site (SRS) Salt Processing Alternatives Supplemental Environmental Impact Statement, identifying Caustic Side Solvent Extraction (CSSX) as the technology to be used for separation of radioactive cesium from SRS high-level waste salt. The CSSX-based Salt Waste Processing Facility (SWPF), currently scheduled to come on line in the next decade, is expected to treat the majority of SRS's salt waste. In order to maintain the ability to prepare sludge for DWPF, to continue DWPF operations, and to assure the availability of necessary tank space for the preparation of salt waste batches destined for SWPF, a number of interim activities are being undertaken. These measures include draining, dissolution, and settling activities done in preparation for dispositioning waste to Saltstone. 
In July of 2003, a federal district court in Idaho declared invalid a portion of a DOE Order that provided guidance for the management of certain waste material in tanks at DOE facilities across the nation. Although DOE has appealed that decision, and has requested that Congress clarify certain federal statutes related to that guidance, DOE is currently re-evaluating disposition pathways for high level waste in light of the district court's decision. This review is being conducted with both SCDHEC and EPA due to the potential impacts to the Waste Removal Plan and Schedule as well as permitting activities needed for accelerated operations at its low level radioactive waste disposal facilities. Short and long term impacts to the Salt Processing program resulting from the district court's decision are inconclusive at this time. The salt treatment and disposal strategy noted in the paragraph above is contingent upon successful resolution of the Idaho lawsuit and the related legislative action currently being considered in Congress.

Except as noted above, no other significant uncertainties (budgetary, permitting, etc.) are identified or anticipated for this composite waste stream at this time. 
THIS PAGE INTENTIONALLY LEFT BLANK. 


\section{Chapter 6. Future Generation of Mixed Waste Streams}

This chapter addresses waste streams generated by Soil and Groundwater Closures Projects and Decontamination and Decommissioning for which specific waste characterization data is needed before an indepth options analysis can be performed. The section explains the types of waste to be generated in future activities at the Savannah River Site (SRS) and the general estimates of those waste volumes.

\subsection{Soil and Groundwater Closures Waste}

The SRS Soil and Groundwater Closures Projects (SGCP) Mission is to remediate inactive waste sites. SRS has implemented a comprehensive environmental program to maintain compliance with environmental regulations and to mitigate impacts to the environment. SGCP activities at SRS are governed by the Federal Facility Agreement (FFA). The FFA is a tri-party agreement among the Department of Energy (DOE), the Environmental Protection Agency (EPA), and the South Carolina Department of Health and Environmental Control (SCDHEC), which became effective on August 16, 1993. The FFA requires that SRS set work priorities on an annual basis with schedules and deadlines for environmental restoration actions. SRS must also submit to EPA and SCDHEC long-term projections including projected deliverable dates for work activities to be conducted over the next two fiscal years and Record of Decision (ROD) dates for the third fiscal year and beyond.

Other SGCP activities are defined by Resource Conservation and Recovery Act (RCRA) permits, closure plans, groundwater corrective action requirements, settlement agreements, and consent decrees. Known mixed wastes for which a clean-up decision is scheduled within the next five years and for which treatment in accordance with the RCRA LDRs may be required are discussed for general planning purposes. Due to the uncertainty of how these ER wastes ultimately will be managed, their inclusion into the Site Treatment Plan (STP) (and therefore the specification of how and when they will be treated) will not occur until a final cleanup decision (under Comprehensive Environmental Response Compensation and Liability Act [CERCLA] or RCRA) has been reached.

If SGCP mixed waste is removed from an area of contamination and is not otherwise subject to an RCRA/CERCLA order or agreement or specifically excluded from the STP, the following actions will be taken to include these waste streams in the STP: (1) review characterization data and obtain more information if necessary to proceed with the preferred option selection process; (2) determine if the new waste would fit into any existing waste stream category by reviewing the waste opposite the characterization information and the preferred treatment option for the existing waste stream; (3) if able to fit into an existing waste stream, modify the MWIR and the STP at the next annual update of the MWIR and STP and proceed with treatment on the same schedule as has been identified for the existing waste stream; (4) if unable to fit this new waste stream into an existing waste category, create a new waste stream and notify SCDHEC within 30 days of discovery as required in the Consent Order, 95-22-HW; (5) identify a preferred treatment option (using the same or similar process as was used to develop other preferred treatment options) and schedule within one year of the notification date.

\subsection{Decontamination and Demolition (D\&D) Waste}

At the end of a facility's lifecycle when it or its mission is no longer needed by DOE, the facility undergoes a process referred to as "disposition." This is a series of stages where the facility is brought from its condition and status at the time operations end to a final end state that involves either dismantlement or conversion for another use. As additional specific projects are funded, walkdowns and initial characterization will be done to generate the best estimated volume and nature of wastes that could be generated. Consequently, D\&D waste will be generated as a result of the following: surveillance and maintenance activities, hazard mitigation activities, deactivation activities, and decommissioning activities. Efforts are currently underway to demolish facilities in A, C, D, E, F, G, H, K, L, M, N, P, R, and T-Areas. It is possible that a new mixed waste stream 
will be identified in this project. If so, SRS will follow the requirements of new mixed waste generation found in consent order 95-22-HW.

\subsection{Additional Waste Streams}

\section{Other Mixed Waste Generated at SRS}

A verbal agreement has been reached with SCDHEC and SRS that waste in satellite accumulation areas that is treated in a 90-day staging area or by elementry neutralization will not be included in the Site Treatment Plan or the Mixed Waste Inventory Report. Exceptions to this agreement are if the waste is continually generated and treated (e.g., SR-W050 supporting ITP process sampling activity) or if the waste is a large quantity (e.g., SR-W072 debris treatment by HLW Operations). These cases are evaluated on a case-by-case basis. 


\section{Chapter 7. (Reserved)}


THIS PAGE INTENTIONALLY LEFT BLANK. 


\section{Chapter 8. Disposal in Support of the Site Treatment Plan Discussions}

This section discusses the overall Department of Energy (DOE) strategy for the disposal of residuals from the treatment of mixed low-level waste (MLLW) subject to the Federal Facility Compliance Act (FFAct). The viable options for the disposal of treatment residuals are provided in this section.

\subsection{Background}

The FFCAct does not impose a requirement for the disposal of mixed wastes after treatment. However, DOE recognizes the need to address this final phase of mixed waste management. Sites identified for mixed waste disposal follow state and federal regulations for siting and permitting and include appropriate public involvement. For waste which treatment capabilities exist but no immediate disposal capabilities exist, the treated waste will be returned to SRS for storage in accordance with RCRA requirements until such time that permanent disposal is available.

Options for disposal of high-level waste are not identified because there are established processes for studying, designing, constructing, and operating disposal facilities for this waste.

DOE has historically planned to develop MLLW disposal facilities at the six DOE sites currently disposing of low-level waste. These sites are Hanford, Savannah River, Oak Ridge Reservation, Idaho National Engineering Laboratory, Nevada Test Site, and Los Alamos National Laboratory. Of these six sites, only two have been developed and are recognized as complex-wide MLLW disposal sites by the final Waste Management Programmatic Environmental Impact Statement (PEIS) Record of Decision (ROD). These sites are Hanford and Nevada Test Site. Along with the two DOE sites, one commercial site, Envirocare of Utah, was developed to dispose of treatment residues.

DOE may initiate site-specific National Environmental Policy Act (NEPA) evaluations for the proposed disposal facilities and initiate performance assessment analyses for compliance with DOE Order 435.1.

\subsection{Current Status of Disposal Sites}

\subsubsection{Hanford Site}

The Hanford Site currently accepts onsite generated mixed wastes that meet Land Disposal Restrictions (LDR). Hanford does not accept offsite mixed waste. The disposal facility consists of two double-lined RCRA Subtitle $\mathrm{C}$ land disposal units with leachate collection. Each trench has a capacity of approximately 21,000 cubic meters. Additional capacity may be available in the future.

Currently SRS does not dispose of any wastes at the Hanford site and there are no active plans to do so.

\subsubsection{Nevada Test Site}

The Nevada Test Site accepts treated mixed waste residues from DOE sites. Offsite and out-of-state generated mixed waste is not accepted. The disposal facility is an unlined pit located at Area 5. The disposal facility operated under RCRA interim status.

The NTS Part B Permit Application must be revised and approved by the State of Nevada prior to accepting offsite or out-of-state mixed waste for disposal. The RCRA permit is pending comment resolution and issuance. 


\subsubsection{Envirocare of Utah}

Envirocare of Utah accepts treated mixed waste residues for Subtitle C disposal. The Utah State Division of Radiation Control and the Division of Solid and Hazardous Waste jointly permit this disposal facility.

Some SRS wastes or residues from the treatment of SRS wastes are being sent to Envirocare for disposal. SRS began sending treated mixed waste to Envirocare in 2001. 


\section{Chapter 9. Treatment Facilities and Treatment Technologies}

This chapter discusses the existing treatment facilities at SRS, the treatment process technologies used for treatment, and technologies that are being considered for the treatment of waste streams in the STP.

\subsection{SRS Facility Descriptions}

This section describes existing SRS treatment facilities considered in the options analysis. The facilities discussed include:

9.1.1 F- and H-Area Effluent Treatment Project (F/H ETP)

9.1.2 (Reserved)

9.1.3 (Reserved)

9.1.4 Saltstone Facility (Z-Area)

9.1.5 Defense Waste Processing Facility (DWPF)

9.1.6 Visual Examination (VE) Facility/Mixed Waste Processing Facility (MWPF)

9.1.7 Mobile Vendor

\subsubsection{F- and H-Area Effluent Treatment Project (F/H ETP)}

F/H ETP is a multi-purpose plant for treating highly dilute aqueous wastes. Waste arrives at F/H ETP by pipeline. A station has also been provided to unload liquid waste for treatment in F/H ETP. The treatment option of interest for treating mixed waste streams is the ion exchange process. However, other treatment components in F/H ETP, carbon adsorption and reverse osmosis, may also be of benefit in treating mixed waste streams.

\section{Facility Description}

A treatability study determines the compatibility of the constituents of concern in the waste with the ion exchange resin that will be used for adsorption. The waste is pumped from the feed tank to the ion exchange beds. The constituents of concern are bound so tightly to the ion exchange resins that studies indicate the resin will pass TCLP, so the spent resin also becomes a non-hazardous low-level waste. Decontaminated liquid effluent is collected in check tanks for analysis, which confirms the liquid meets release specifications. Liquid that meets specifications is released to a surface outfall. In the unlikely event that the treated effluent fails to meet release specification, it can easily be recycled to the feed system for reprocessing. Nothing is released from F/H ETP without passing a final assay.

\section{Capacity}

Demonstrated maximum throughput of F/H ETP is about 130 gallons per minute (gpm). At present F/H ETP is processing about 40-50 gpm average. Acceptance of waste streams at F/H ETP must be on a case-by-case basis, depending on the quantity of waste and concentration of the constituent of concern.

\subsection{2 (Reserved)}

\subsection{3 (Reserved)}

\subsubsection{Saltstone Facility (Z-Area)}

\section{Facility Description}


The Z-Area Saltstone Facility immobilizes and disposes of waste salt solution from F and H-Area Tank Farms. The salt solution is mixed with a dry material mixture consisting of cement, slag, and flyash to form a grout. The resulting grout is disposed of by transferring it to an engineered concrete vault where it is allowed to harden. The saltstone waste form is classified as low-level waste.

Saltstone is permitted by SCDHEC as an Industrial Waste Landfill that is to be used only for the disposal of non-hazardous low-level solid waste (saltstone). Once formed into saltstone, the radionuclides and the toxic chemicals in the salt solution are immobilized and isolated from the environment by the stability of the material and the design of the vaults.

\section{Capacity}

The capacity of the facility is not to exceed a daily rate of 140,000 gallons per day. This limit is found in the wastewater treatment permit for the mixing plant.

\subsubsection{Defense Waste Processing Facility (DWPF)}

DWPF is currently receiving high-level sludge waste from the tank farms. This waste includes liquids, sludge, and precipitated materials in a slurry from reprocessing spent nuclear fuel and plutonium targets. DWPF is not receiving salt waste at this time because work on salt processing was suspended in January 1998 due to technical issues with the In-Tank Precipitation (ITP) Facility in January 1998.

\section{Facility Description}

A chemical process treats the high-level radioactive aqueous stream and sludge to remove mercury, mixes the streams with additives and glass-forming materials, and continuously feeds a high-temperature melter in which the materials are vitrified into borosilicate glass.

\section{Capacity}

Actual capacity may be significantly impacted by required outages for melter replacement and control system upgrades. Melter number one was changed out in the winter of 2002 which impacted the plant availability for CY 2002 and CY2003. SRS HLW will be treated in accordance with the treatment schedule identified in Chapter 5 of Volume I of the SRS Approved Site Treatment Plan.

\subsubsection{Visual Examination (VE) Facility/Mixed Waste Processing Facility (MWPF)}

The Visual Examination (VE) Facility has been merged to include the TRU and MW sorting activities into one facility for characterization and repackaging of waste. The VE Facility is a stainless steel, radioactive containment building consisting of two airlocks, secondary and tertiary confinement rooms, a glovebox to provide primary confinement, and a ventilation system.

The VE Facility, which began operations during 4QFY00, provides capabilities to visually confirm the physical characteristics of the TRU waste as compared with the radiography results, sort, and repackage $>100 \mathrm{nCi} / \mathrm{g}$ and 10-100 nCi/g mixed and non-mixed TRU wastes. The waste types include job control waste, process equipment, and miscellaneous debris from production, D\&D, and ER activities.

In the Mixed Waste Processing Facility (MWPF) portion of this facility, containers are opened, and WIPPprohibited items are removed.

\subsubsection{Mobile Vendor}

SRS utilizes a Mobile Vendor for the inspection/characterization and shipment of TRU waste from SRS to WIPP. The Mobile Vendor mobilized in 2QFY01 and began characterization operations in 4QFY01. The 
Mobile Vendor is expected to process and ship waste from SRS to WIPP through FY06. The Mobile Vendor provides this characterization and shipping capability through the use of four mobile systems. The first is a real time radiography trailer that is used to x-ray TRU drums to determine drum contents and waste attributes. Following x-ray the drums are processed through one of two non-destructive assay trailers. One system uses an integrated gamma and imaging passive/active neutron/gamma energy analysis (IPAN/GEA) mobile waste assay trailer. The IPAN/GEA provides information to determine the isotopic composition of each TRU waste drum. A second system uses gamma spectroscopy (IQ3). After assay the drums are placed into heated sealand storage containers for 72 hours prior to head space gas sampling. The fourth mobile system is the drum headspace gas sampling system. This system utilizes a gas chromatograph (GC) and mass spectrometer (MS) to analyze the constituents of the TRU drum headspace gases. The drums are then moved to the final system which is the mobile loading unit. This system contains the adjustable center gravity lift fixture, shrink wrap machine and equipment necessary to configure and load TRU drums into TRUPACT-II containers for transport to WIPP for disposal. These systems are currently set-up on existing TRU waste storage Pads \#4 and \#3, although future systems, such as additional radiography trailers, may be set up near the TRU pads. In these situations, containers are transported one at a time for appropriate screening or analysis and returned to the TRU pads. This process is supported by existing SRS infrastructure to include the Visual Examination Facility currently on TRU Pad \#6.

\subsubsection{Modular Repackaging Facility}

The Modular Repackaging Facility, currently on Pad 19, is a hazard category II facility that will sort and segregate TRU waste contained in drums. It will remove prohibited items and will repackage waste to meet transportation limits for shipment to WIPP for disposal.

\subsection{Process Descriptions}

This section contains descriptions of the treatment technologies considered in the options analysis. These processes are commercially available for onsite or offsite use and include the following:

$\begin{array}{ll}\text { 9.2.1 } & \text { Amalgamation } \\ \text { 9.2.2 } & \text { Combustion } \\ \text { 9.2.3 } & \text { Filtration } \\ \text { 9.2.4 } & \text { Immobilization } \\ \text { 9.2.5 } & \text { Ion Exchange } \\ \text { 9.2.6 } & \text { Macroencapsulation } \\ \text { 9.2.7 } & \text { Decontamination of Lead } \\ \text { 9.2.8 } & \text { Neutralization } \\ \text { 9.2.9 } & \text { Precipitation } \\ \text { 9.2.10 } & \text { Pretreatment Process } \\ \text { 9.2.11 } & \text { Roasting/Retorting } \\ \text { 9.2.12 } & \text { Stabilization } \\ \text { 9.2.13 } & \text { Thermal Treatment } \\ \text { 9.2.14 } & \text { Vitrification }\end{array}$

\subsubsection{Amalgamation}

Amalgamation is a process applicable to waste liquid, elemental mercury contaminated with radioactive materials. Mercury is combined with inorganic reagents such as copper, zinc, nickel, gold, or sulfur that results in a non-liquid semi-solid amalgam, which is more easily managed and less mobile. 


\subsubsection{Combustion}

Combustion is incinerators, boilers, or industrial furnaces operated in accordance with the applicable requirements of Part 264 of SCHWMR, Subpart O, or Part 266 SCHWMR, Subpart H.

\subsubsection{Filtration}

Filtration is removal/separation of particles from a mixture of fluid and particles by a medium that permits the flow of the fluid but retains the particles. Usually, the larger the particles, the easier they are to remove from the fluid. Chemicals called flocculants may be mixed with the fluids to increase the size of the particles and make it easier to separate particles from the fluid.

\subsubsection{Immobilization}

Immobilization is treatment of waste through macroencapsulation, microencapsulation, or sealing to reduce surface exposure to potential leaching media or to reduce the leachability of the hazardous constituents.

\subsubsection{Ion Exchange}

Ion exchange uses a resin to replace certain specific ions in a solution with other ions that are innocuous. Ion exchange is used to separate a mixed waste into its radioactive and hazardous constituents if the components are ionic. It will also concentrate the radioactive ionic species into a small volume on an organic polymec (ion exchange resin), leaving a less radioactive aqueous phase. The principal mixed waste application of this process is to recover metallic radionuclides from wastewaters or acid leach liquids.

\subsubsection{Macroencapsulation}

One type of macroencapsulation is immobilization by application of surface coating materials such as polymeric organics (e.g., resins and plastics) or a jacket of inert inorganic materials to substantially reduce surface exposure to potential leaching media. Another type of macroencapsulation is immobilization by enclosing the waste in a specially designed container that substantially reduces surface exposure to potentially leaching media.

\subsubsection{Decontamination of Lead}

Elemental lead waste can be decontaminated by immersion in an acid bath. The acid dissolves the surface of the lead, which has been contaminated with radionuclides. The decontaminated lead can then be washed and reused. The acid solution is neutralized and the dissolved lead is precipitated. The precipitate is removed and stabilized for disposal. The neutralized solution can be further treated for reuse or recycle. Other methods of lead decontamination may involve scouring the lead surface with an abrasive or simply slicing away the contaminated surface layer.

\subsubsection{Neutralization}

Neutralization uses these chemicals alone or in combination; acids, bases, or water (including wastewaters) resulting in a $\mathrm{pH}$ greater than 2 but less than 12.5 as measured in the aqueous residuals.

\subsubsection{Precipitation}

Precipitation removes metals and other inorganics by forming insoluble compounds of oxides, hydrides, carbonates, sulfides, sulfates, chlorides, fluorides, or phosphates. These precipitants are typically used alone or in combination: lime (i.e., containing oxides and/or hydroxides of calcium and/or magnesium); caustic (i.e., 
sodium and/or potassium hydroxides); soda ash (i.e., sodium carbonate); sodium sulfide; ferric sulfate or ferric chloride; alum; or sodium sulfate. Additional chemicals for flocculating and coagulating precipitates to enhance sludge dewatering may also be used.

\subsubsection{Pretreatment Process}

Processes (e.g., shredding, grinding, physical separation, repackaging, volume, reduction, etc.,) that make the waste amenable to the treatment process that ultimately destroys, removes, or immobilizes the hazardous contaminants or characteristics.

\subsubsection{Roasting/Retorting}

Roasting and retorting mercury from radioactive contaminated process equipment has two major components as explained below.

\section{Mercury Oven (Roaster)}

The mercury oven is electrically heated to approximately $400^{\circ} \mathrm{C}$ with a mechanical vacuum pump providing the required vacuum or negative pressure. At this temperature, elemental mercury is vaporized and driven into the offgas stream of the roaster.

\section{Condenser/Decanter (Retort)}

The condenser is connected to the offgas system from the oven to condense the mercury vapor and vaporized organic compounds. The mercury is drawn off the bottom of the condenser receiver. Liquid organics are decanted at the supernatant interface. The gas coming out of the condenser may be exhausted through the offgas system or receive further treatment.

\subsubsection{Stabilization}

Stabilization comprises treatment processes that immobilize hazardous constituents in a waste. For treatment of metals in mixed low-level wastes, stabilization technologies will reduce the leachability of the hazardous metal constituents (regardless of whether the metals are radioactive) in nonwastewater matrices. Stabilization usually involves the use of Portland $®$ cement, lime pozzolans, iron salts, silicates or clays as treatment reagents.

\subsubsection{Thermal Treatment}

Thermal treatment is the physical separation of organics from solid materials by the use of thermal desorption or other thermal separation methods followed by combustion of organics. Remaining solids will be macroed or shredded and stabilized.

\subsubsection{Vitrification}

Vitrification is a waste treatment process in which waste is mixed with glass and fused into a solid mass. The resultant mass is expected to remain a stable and insoluble form for long time periods. (Vitrification with borosilicate glass is the specified LDR treatment standard for HLW and certain mixed waste streams.) 


\subsection{Planned/Proposed Facilities or Technologies}

\subsubsection{Solidification}

Solidification is a technology to immobilize hazardous constituents in organic waste by physical and chemical methods. Optimum ingredients and formulations are being developed.

\subsubsection{Solvent Extraction}

Caustic Side Solvent Extraction uses an organic extractant to separate cesium from HLW salt solutions. The cesium would be transferred into an insoluble organic phase using a centrifugal contactor, which will provide high surface area contact. The cesium would then be recovered from the organic phase and sent to DWPF. 


\section{Chapter 10. Offsite Waste Streams for which SRS is the PREFERRED OPTION}

Naval Reactors (NR) had selected the SRS Consolidated Incineration Facility (CIF) as a preferred option in the Naval Reactors Program STPs. The waste at SRS that was received from CIF will now be sent offsite to a commercial vendor, unless sent to other facilities for treatment.

Shipment has been received for Charleston Naval Shipyard waste by SRS, and the waste is currently in RCRA regulated storage. Bettis waste was shipped to SRS March 1999.

SRS will receive Naval Reactor's program mixed waste only upon SCDHEC approval, as described in consent order 95-22-HW. No requests for approval have been sent to SCDHEC since 1999. Other waste described below includes future MTRU waste shipments from DOE's Mound Site (see Vol. I, Sec. 4.2.2).

Table 10.1 Offsite Waste Streams

\begin{tabular}{|c|l|l|c|c|}
\hline $\begin{array}{c}\text { Waste } \\
\text { Stream No. }\end{array}$ & \multicolumn{1}{|c|}{ Site } & Waste Stream & $\begin{array}{c}\text { SRS } \\
\text { Treatment } \\
\text { Facility }\end{array}$ & $\begin{array}{c}\text { Current } \\
\text { Cumulative } \\
\text { Inventory through } \\
\left.\mathbf{9 / 3 0 / 0 4} \mathbf{( m}^{3}\right)\end{array}$ \\
\hline SR-W080 & $\begin{array}{l}\text { Charleston Naval } \\
\text { Shipyard (CN- } \\
\text { W001, CN-W004) }\end{array}$ & $\begin{array}{l}\text { Solids } \\
\text { containing } \\
\text { Potassium } \\
\text { Chromate; } \\
\text { Organic Debris } \\
\text { containing Lead } \\
\text { and/or } \\
\text { Chromium }\end{array}$ & $\begin{array}{l}\text { Offsite } \\
\text { Commercial } \\
\text { Vendor }\end{array}$ & 0.0* \\
\hline SR-W087 & $\begin{array}{l}\text { Bettis Atomic } \\
\text { Power Laboratory } \\
\text { Contaminated Oil } \\
\text { (BT-W035, part } \\
\text { of BT-W003) }\end{array}$ & $\begin{array}{l}\text { Oil containing } \\
\text { uranium, } \\
\text { cadmium, and } \\
\text { lead }\end{array}$ & $\begin{array}{l}\text { Offsite } \\
\text { Commercial } \\
\text { Vendor }\end{array}$ & \\
\hline SR-W089 & Mound & $\begin{array}{l}\text { TRU Mixed } \\
\text { Waste }\end{array}$ & $\begin{array}{l}\text { TRU } \\
\text { Facilities } \\
\text { (prepare for } \\
\text { shipment to } \\
\text { WIPP) }\end{array}$ & \\
\hline
\end{tabular}

* Cumulative inventory through 9/30/04

** No future mixed waste will be generated from the Charleston Naval Shipyard since that facility was closed in April 1996. SR-W80 has been shipped for treatment. 
THIS PAGE INTENTIONALLY LEFT BLANK. 


\section{Chapter 11. Volume Summary Information}

This chapter includes volume and treatment residue summaries for the waste streams listed in the STP. Table 11.1 lists the volume summary of each waste stream by its unique waste stream number. Table 11.2 lists the volume summary for each waste stream by treatment facility. Volume summaries include inventories as of September 30, 2004. Table 11.3 shows the volume and status of mixed treatment residues for those waste streams that have been treated and the residues must continue to be managed as a mixed waste. The following waste streams have undergone treatment at the M-Area Vendor Treatment Facility: SR-W005, Mark 15 Filtercake; SR-W029, M-Area Sludge Treatability Samples; SR-W031, Uranium/Chromium Solution; SRW037, M-Area Plating Line Solution; SR-W038, Plating Line Sump Material; SR-W039, Nickel Plating Line Solution; SR-W048, Soils from Spill Remediation; and SR-W082, Radioactive Chemicals, Metals, and Pesticides (CMP) Soils.

\section{Table 11.1 Volume Summary by Waste Stream Number}

\begin{tabular}{|c|c|c|c|}
\hline $\begin{array}{c}\text { Waste } \\
\text { Stream No. }\end{array}$ & Waste Stream Name & Preferred Option (PO) & $\begin{array}{c}\text { Current } \\
\text { Cumulative } \\
\text { Inventory } \\
\text { through } \\
09 / 30 / 04 \\
\left(\mathrm{~m}^{3}\right) \\
\end{array}$ \\
\hline SR-W001 & $\begin{array}{l}\text { Rad-Contaminated } \\
\text { Solvents }\end{array}$ & $\begin{array}{l}\text { Combustion at commercial } \\
\text { facility }\end{array}$ & 26.4 \\
\hline SR-W002 & $\begin{array}{l}\text { Rad-Contaminated } \\
\text { Chlorofluorocarbons }\end{array}$ & $\begin{array}{l}\text { Consolidated with } \\
\text { SR-W001 }\end{array}$ & N/A \\
\hline SR-W003 & $\begin{array}{l}\text { Solvent Contaminated } \\
\text { Debris (LLW) }\end{array}$ & $\begin{array}{l}\text { Thermal Treatment, } \\
\text { macroencapsulation, or } \\
\text { stabilization and/or } \\
\text { alternative debris } \\
\text { treatment at commercial } \\
\text { vendor facility }\end{array}$ & 0.7 \\
\hline SR-W004 & $\begin{array}{l}\text { M-Area Plating Line } \\
\text { Sludge from Supernate } \\
\text { Treatment }\end{array}$ & $\begin{array}{l}\text { Consolidated with SR- } \\
\text { W037 }\end{array}$ & N/A \\
\hline SR-W005 & Mark 15 Filtercake & $\begin{array}{l}\text { Stabilization by } \\
\text { Vitrification - M-Area } \\
\text { Vendor Treatment Facility }\end{array}$ & 0.0 \\
\hline SR-W006 & CH Mixed TRU/Liquids & $\begin{array}{l}\text { Characterization at SRS- } \\
\text { WIPP Disposal }\end{array}$ & 0.1 \\
\hline SR-W007 & SRNL Sample Material* & Waste stream eliminated* & N/A \\
\hline SR-W008 & $\begin{array}{l}\text { Separations Area Sample } \\
\text { Receipts from SRNL* }\end{array}$ & $\begin{array}{l}\text { Vitrification, Stabilization, } \\
\text { or WW Treatment* }\end{array}$ & $\begin{array}{c}* \\
36.7\end{array}$ \\
\hline SR-W009 & $\begin{array}{l}\text { Silver Coated Packing } \\
\text { Material }\end{array}$ & $\begin{array}{l}\text { Macroencapsulation in a } \\
\text { Steel Container -Onsite } \\
\text { via a Treatability Variance }\end{array}$ & $6.3^{*}$ \\
\hline SR-W010 & Scintillation Solution & $\begin{array}{l}\text { Consolidated with } \\
\text { SR-W001 }\end{array}$ & N/A \\
\hline SR-W011 & $\begin{array}{l}\text { Cadmium Coated HEPA } \\
\text { Filters }\end{array}$ & Scrap Metal Exclusion & 0.0 \\
\hline SR-W012 & Toxic Characteristic Solids & $\begin{array}{l}\text { Thermal Treatment, } \\
\text { Macroencapsulation, or } \\
\text { stabilization and/or } \\
\text { alternative debris } \\
\text { treatment at commercial } \\
\text { facility }\end{array}$ & 12.5 \\
\hline
\end{tabular}




\section{Table 11.1 Volume Summary by Waste Stream Number (con't)}

\begin{tabular}{|c|c|c|c|}
\hline $\begin{array}{c}\text { Waste } \\
\text { Stream No. }\end{array}$ & Waste Stream Name & Preferred Option (PO) & $\begin{array}{c}\text { Current } \\
\text { Cumulative } \\
\text { Inventory } \\
\text { through } \\
09 / 30 / 04 \\
\left(\mathrm{~m}^{3}\right)\end{array}$ \\
\hline SR-W013 & $\begin{array}{l}\text { Low-Level Waste (LLW) } \\
\text { Lead-to be } \\
\text { Decontaminated Onsite }\end{array}$ & $\begin{array}{l}\text { Reclassified as material in } \\
\text { use }\end{array}$ & N/A \\
\hline SR-W014 & $\begin{array}{l}\text { Elemental (Liquid) } \\
\text { Mercury - Sitewide }\end{array}$ & $\begin{array}{l}\text { Amalgamation-Offsite } \\
\text { Commercial Vendor }\end{array}$ & 0.6 \\
\hline SR-W015 & $\begin{array}{l}\text { Tritium Contaminated } \\
\text { Equipment }\end{array}$ & $\begin{array}{l}\text { Macroencapsulation in S. } \\
\text { S. Container as 90-Day } \\
\text { Generator }\end{array}$ & 22.0 \\
\hline SR-W016 & $\begin{array}{l}\text { 221-F Canyon High-Level } \\
\text { Liquid Waste }\end{array}$ & Vitrification at DWPF & $\begin{array}{c}* \\
52,500 \\
\end{array}$ \\
\hline SR-W017 & $\begin{array}{l}\text { 221-H Canyon High-Level } \\
\text { Liquid Waste }\end{array}$ & Vitrification at DWPF & $\begin{array}{c}* \\
85,400\end{array}$ \\
\hline SR-W018 & Listed Incinerable Solids & $\begin{array}{l}\text { Combustion at commercial } \\
\text { facility or } \\
\text { macroencapsulation or } \\
\text { stabilization at commercial } \\
\text { facility }\end{array}$ & 0.0 \\
\hline SR-W019 & $\begin{array}{l}\text { 244-H RBOF High } \\
\text { Activity Liquid Waste }\end{array}$ & $\begin{array}{l}\text { Consolidated with } \\
\text { SR-W017 }\end{array}$ & N/A \\
\hline SR-W020 & Salt Processing Filters & $\begin{array}{l}\text { Future Generation - } \\
\text { Treatment to be } \\
\text { Determined }\end{array}$ & 0.0 \\
\hline SR-W021 & Poisoned Catalyst Material & Waste stream eliminated & N/A \\
\hline SR-W022 & DWPF Benzene & Waste stream eliminated & N/A \\
\hline SR-W023 & $\begin{array}{l}\text { Cadmium Safety/Control } \\
\text { Rods }\end{array}$ & $\begin{array}{l}\text { Macroencapsulation in a } \\
\text { cask as a 90-day generator }\end{array}$ & $3.5^{*}$ \\
\hline SR-W024 & $\begin{array}{l}\text { Mercury/Tritium Gold } \\
\text { Traps }\end{array}$ & $\begin{array}{l}\text { Meets LDR Treatment } \\
\text { Standard }\end{array}$ & 3.0 \\
\hline SR-W025 & $\begin{array}{l}\text { Solvent /TRU Job Control } \\
\text { Waste }<100 \mathrm{nCi} / \mathrm{g}\end{array}$ & $\begin{array}{l}\text { Consolidated with SR- } \\
\text { W027 }\end{array}$ & N/A \\
\hline SR-W026 & CH Mixed TRU/Thirds & $\begin{array}{l}\text { Characterization at SRS- } \\
\text { WIPP Disposal }\end{array}$ & 175.6 \\
\hline SR-W027 & $\begin{array}{l}\text { CH Mixed TRU/F-listed } \\
\text { Solvents }\end{array}$ & $\begin{array}{l}\text { Characterization at SRS- } \\
\text { WIPP Disposal }\end{array}$ & 4574.4 \\
\hline SR-W028 & Mark 15 Filter Paper & $\begin{array}{l}\text { Combustion at commercial } \\
\text { facility or } \\
\text { macroencapsulation or } \\
\text { stabilization at commercial } \\
\text { facility }\end{array}$ & 0.0 \\
\hline SR-W029 & $\begin{array}{l}\text { M-Area Sludge } \\
\text { Treatability Samples }\end{array}$ & Waste Stream Treated & 0.0 \\
\hline SR-W030 & Spent Methanol Solution & $\begin{array}{l}\text { Consolidated with } \\
\text { SR-W001 }\end{array}$ & N/A \\
\hline
\end{tabular}


Table 11.1 Volume Summary by Waste Stream Number (con't)

\begin{tabular}{|c|c|c|c|}
\hline $\begin{array}{c}\text { Waste } \\
\text { Stream No. }\end{array}$ & Waste Stream Name & Preferred Option (PO) & $\begin{array}{c}\text { Current } \\
\text { Cumulative } \\
\text { Inventory } \\
\text { through } \\
09 / 30 / 04 \\
\left(\mathrm{~m}^{3}\right)\end{array}$ \\
\hline SR-W031 & $\begin{array}{l}\text { Uranium/Chromium } \\
\text { Solution }\end{array}$ & $\begin{array}{l}\text { Stabilization by } \\
\text { Vitrification M-Area } \\
\text { Vendor Treatment Facility }\end{array}$ & 0.0 \\
\hline SR-W032A & $\begin{array}{l}\text { Mercury Contaminated } \\
\text { Heavy Water }\end{array}$ & Waste Stream Eliminated & N/A \\
\hline SR-W032B & $\begin{array}{l}\text { Mercury-Contaminated } \\
\text { Heavy Water Residues }\end{array}$ & $\begin{array}{l}\text { Solidification in container } \\
\text { as a 90-day generator }\end{array}$ & 0.0 \\
\hline SR-W033 & $\begin{array}{l}\text { Thirds/TRU Job Control } \\
\text { Waste }<100 \mathrm{nCi} / \mathrm{g}\end{array}$ & $\begin{array}{l}\text { Consolidated with SR- } \\
\text { W026* }\end{array}$ & N/A \\
\hline SR-W034 & Calcium Metal & $\begin{array}{l}\text { Deactivation by } \\
\text { commercial vendor }\end{array}$ & 0.0 \\
\hline SR-W035 & Mixed Waste Oil-Sitewide & $\begin{array}{l}\text { Thermal Treatment at } \\
\text { commercial facility }\end{array}$ & 27.8 \\
\hline SR-W036 & Tritiated Oil with Mercury & $\begin{array}{l}\text { Treatment by aging } \\
\text { followed by Combustion }\end{array}$ & 18.1 \\
\hline SR-W037 & $\begin{array}{l}\text { M-Area Plating Line } \\
\text { Sludges }\end{array}$ & $\begin{array}{l}\text { Stabilization by } \\
\text { Vitrification M-Area } \\
\text { Vendor Treatment Facility }\end{array}$ & 0.0 \\
\hline SR-W038 & $\begin{array}{l}\text { Plating Line Sump } \\
\text { Material }\end{array}$ & $\begin{array}{l}\text { Stabilization by } \\
\text { Vitrification M-Area } \\
\text { Vendor Treatment Facility }\end{array}$ & 0.0 \\
\hline SR-W039 & $\begin{array}{l}\text { Nickel Plating Line } \\
\text { Solution }\end{array}$ & $\begin{array}{l}\text { Stabilization by } \\
\text { Vitrification M-Area } \\
\text { Vendor Treatment Facility }\end{array}$ & 0.0 \\
\hline SR-W040 & $\begin{array}{l}\text { Listed Stabilized } \\
\text { Sludge/Listed LDR- } \\
\text { Compliant Debris }\end{array}$ & $\begin{array}{l}\text { Waste Stream Treated in } \\
\text { Compliance with LDR }\end{array}$ & 18.0 \\
\hline SR-W041 & $\begin{array}{l}\text { Aqueous Mercury and } \\
\text { Lead }\end{array}$ & Effluent Treatment Project & 0.0 \\
\hline SR-W042 & Paints and Thinners & $\begin{array}{l}\text { Thermal Treatment, } \\
\text { macroencapsulation, or } \\
\text { stabilization at commercial } \\
\text { facility }\end{array}$ & 0.9 \\
\hline SR-W043 & $\begin{array}{l}\text { Lab Waste w/Tetraphenyl } \\
\text { Borate }\end{array}$ & $\begin{array}{l}\text { Consolidated with } \\
\text { SR-W012 }\end{array}$ & N/A \\
\hline SR-W044 & $\begin{array}{l}\text { Tri-Butyl-Phosphate \& n- } \\
\text { Paraffin - TRU }\end{array}$ & $\begin{array}{l}\text { Consolidated with } \\
\text { SR-W045 }\end{array}$ & N/A \\
\hline SR-W045A & PUREX Aqueous Waste & Waste stream eliminated & N/A \\
\hline SR-W045 & PUREX Waste & $\begin{array}{l}\text { Direct Solidification using } \\
\text { New technology }\end{array}$ & 88.0 \\
\hline SR-W046 & $\begin{array}{l}\text { Consolidated Incineration } \\
\text { Facility Ash }\end{array}$ & Waste stream eliminated & N/A \\
\hline SR-W047 & $\begin{array}{l}\text { Consolidated Incineration } \\
\text { Facility Blowdown }\end{array}$ & Waste stream eliminated & N/A \\
\hline SR-W048 & $\begin{array}{l}\text { Soils from Spill } \\
\text { Remediation }\end{array}$ & $\begin{array}{l}\text { Stabilization by } \\
\text { Commercial Vendor }\end{array}$ & 0.0 \\
\hline
\end{tabular}




\section{Table 11.1 Volume Summary by Waste Stream Number (con't)}

\begin{tabular}{|c|c|c|c|}
\hline $\begin{array}{c}\text { Waste } \\
\text { Stream No. }\end{array}$ & Waste Stream Name & Preferred Option (PO) & $\begin{array}{c}\text { Current } \\
\text { Cumulative } \\
\text { Inventory } \\
\text { through } \\
09 / 30 / 04 \\
\left(\mathbf{m}^{3}\right) \\
\end{array}$ \\
\hline SR-W049 & $\begin{array}{l}\text { Tank E-3-1 Clean Out } \\
\text { Material }\end{array}$ & Waste stream eliminated & N/A \\
\hline SR-W050 & $\begin{array}{l}\text { Waste to Support High- } \\
\text { Level Waste (HLW) } \\
\text { Processing Demonstrations }\end{array}$ & $\begin{array}{l}\text { Treatment by SRNL as a } \\
\text { 90-Day Generator }\end{array}$ & 0.1 \\
\hline SR-W051 & $\begin{array}{l}\text { Spent Filter Cartridges and } \\
\text { Carbon Filter Media }\end{array}$ & $\begin{array}{l}\text { Thermal Treatment, } \\
\text { macroencapsulation, or } \\
\text { stabilization and/or } \\
\text { alternative debris } \\
\text { treatment at commercial } \\
\text { facility }\end{array}$ & 3.8 \\
\hline SR-W052 & $\begin{array}{l}\text { Cadmium Contaminated } \\
\text { Glovebox Section }\end{array}$ & Waste stream eliminated & N/A \\
\hline SR-W053 & Rocky Flats Ash & $\begin{array}{l}\text { Characterization at SRS- } \\
\text { Ship to WIPP }\end{array}$ & 0.1 \\
\hline SR-W054 & $\begin{array}{l}\text { Enriched Uranium } \\
\text { Contaminated with Lead }\end{array}$ & $\begin{array}{l}\text { Consolidated with } \\
\text { SR-W037 }\end{array}$ & N/A \\
\hline SR-W055 & $\begin{array}{l}\text { Job Control Waste } \\
\text { Containing Solvent } \\
\text { Contaminated Wipes }\end{array}$ & $\begin{array}{l}\text { Thermal Treatment at } \\
\text { commercial facility or } \\
\text { macroencapsulation, } \\
\text { stabilization or alternate } \\
\text { debris treatment at } \\
\text { commercial facility } \\
\end{array}$ & 0.0 \\
\hline SR-W056 & $\begin{array}{l}\text { Job Control Waste with } \\
\text { Enriched Uranium and } \\
\text { Solvent Contaminated } \\
\text { Wipes }\end{array}$ & $\begin{array}{l}\text { Waste stream re- } \\
\text { characterized }\end{array}$ & N/A \\
\hline SR-W057 & $\begin{array}{l}\text { D-Tested Neutron } \\
\text { Generators }\end{array}$ & Waste stream eliminated & N/A \\
\hline SR-W058 & $\begin{array}{l}\text { Mixed Sludge Waste with } \\
\text { Mercury from DWPF } \\
\text { Treatability Studies }\end{array}$ & $\begin{array}{l}\text { Treatment by SRNL as a } \\
\text { 90-Day Generator }\end{array}$ & 0.0 \\
\hline SR-W059 & Tetrabutyl Titanate (TBT) & $\begin{array}{l}\text { Consolidated with } \\
\text { SR-W001 }\end{array}$ & N/A \\
\hline SR-W060 & $\begin{array}{l}\text { Tritiated Water with } \\
\text { Mercury }\end{array}$ & $\begin{array}{l}\text { Macroencapsulation in a } \\
\text { Steel Container via a } \\
\text { Treatability Variance }\end{array}$ & $0.4 *$ \\
\hline SR-W061 & DWPF Mercury & $\begin{array}{l}\text { Consolidated with SR- } \\
\text { W068 }\end{array}$ & N/A \\
\hline SR-W062 & $\begin{array}{l}\text { Normal Low-Level } \\
\text { Contaminated Debris }\end{array}$ & $\begin{array}{l}\text { Macroencapsulation or } \\
\text { stabilization by a } \\
\text { Commercial Vendor } \\
\text { and/or alternative debris } \\
\text { treatment at an Offsite } \\
\text { Facility }\end{array}$ & 10.8 \\
\hline SR-W063 & $\begin{array}{l}\text { Macroencapsulated Low- } \\
\text { Level Waste }\end{array}$ & Meets Treatment Standard & 0.0 \\
\hline
\end{tabular}


Table 11.1 Volume Summary by Waste Stream Number (con't)

\begin{tabular}{|c|c|c|c|}
\hline $\begin{array}{c}\text { Waste } \\
\text { Stream No. }\end{array}$ & Waste Stream Name & Preferred Option (PO) & $\begin{array}{c}\text { Current } \\
\text { Cumulative } \\
\text { Inventory } \\
\text { through } \\
09 / 30 / 04 \\
\left(\mathbf{m}^{3}\right) \\
\end{array}$ \\
\hline SR-W064 & $\begin{array}{l}\text { IDW } \\
\text { Soils/Sludges/Slurries }\end{array}$ & Awaiting ROD, etc. & 0.0 \\
\hline SR-W065 & $\begin{array}{l}\text { IDW Monitoring Well } \\
\text { Purge/Development Water }\end{array}$ & Awaiting ROD, etc. & 0.0 \\
\hline SR-W066 & IDW Debris & Awaiting ROD, etc. & 0.0 \\
\hline SR-W067 & $\begin{array}{l}\text { IDW Personal Protective } \\
\text { Equipment (PPE) Waste }\end{array}$ & Awaiting ROD, etc. & 0.0 \\
\hline SR-W068 & $\begin{array}{l}\text { Elemental (Liquid) } \\
\text { Mercury-Sitewide }\end{array}$ & $\begin{array}{l}\text { Consolidated with SR- } \\
\text { W014* }\end{array}$ & N/A \\
\hline SR-W069 & $\begin{array}{l}\text { Low-Level Waste (LLW) } \\
\text { Lead-to be } \\
\text { Macroencapsulated Offsite }\end{array}$ & $\begin{array}{l}\text { Macroencapsulation by a } \\
\text { Vendor at an Offsite } \\
\text { Facility }\end{array}$ & 124.6 \\
\hline SR-W070 & $\begin{array}{l}\text { Mixed Waste from } \\
\text { Laboratory Samples }\end{array}$ & $\begin{array}{l}\text { Combustion at } \\
\text { Commercial Vendor } \\
\text { Facility or } \\
\text { Macroencapsulation or } \\
\text { Stabilization at } \\
\text { Commercial Vendor } \\
\text { Facility }\end{array}$ & 39.8 \\
\hline SR-W071 & $\begin{array}{l}\text { Wastewater Suitable for } \\
\text { thermal treatment or } \\
\text { stabilization }\end{array}$ & $\begin{array}{l}\text { Thermal Treatment or } \\
\text { stabilization at commercial } \\
\text { vendor facility }\end{array}$ & 0.8 \\
\hline SR-W072 & $\begin{array}{l}\text { Supernate or Sludge } \\
\text { Contaminated Debris from } \\
\text { High-Level Waste (HLW) } \\
\text { Operations }\end{array}$ & $\begin{array}{l}\text { Extraction or } \\
\text { Immobilization } \\
\text { Alternative Debris } \\
\text { Technologies as 90-day } \\
\text { Generator }\end{array}$ & 0.0 \\
\hline SR-W073 & $\begin{array}{l}\text { Cadmium-Containing } \\
\text { Raschig Rings }\end{array}$ & $\begin{array}{l}\text { Macroencapsulation by } \\
\text { Commercial Vendor } \\
\text { Offsite via Treatability } \\
\text { Variance or stabilization at } \\
\text { Commercial Vendor } \\
\text { Facility }\end{array}$ & 0.0 \\
\hline SR-W077 & $\begin{array}{l}\text { Aqueous Characteristic } \\
\text { Wastewater }\end{array}$ & $\begin{array}{l}\text { Ion Exchange, Filtration, } \\
\text { and/or Stabilization at F/H } \\
\text { ETP, Saltstone, or } \\
\text { Commercial Vendor }\end{array}$ & 5.5 \\
\hline SR-W078 & $\begin{array}{l}\text { LDR Hazardous Waste } \\
\text { Awaiting Radiological } \\
\text { Screening }\end{array}$ & Waste Stream Eliminated & N/A \\
\hline SR-W079 & $\begin{array}{l}\text { Polychlorinated Biphenyl } \\
\text { (PCB) Mixed Waste }\end{array}$ & $\begin{array}{l}\text { Combustion at the ETTP } \\
\text { TSCA Incinerator or } \\
\text { Vendor Alternate Debris } \\
\text { Technology Treatment }\end{array}$ & 0.0 \\
\hline $\begin{array}{l}\text { SR-W080 } \\
\text { (CN-W001, } \\
\text { CN-W004) }\end{array}$ & $\begin{array}{l}\text { Charleston Naval Shipyard } \\
\text { Waste }\end{array}$ & $\begin{array}{l}\text { Thermal Treatment or } \\
\text { macroencapsulation, or } \\
\text { stabilization at commercial } \\
\text { vendor }\end{array}$ & 0.0 \\
\hline
\end{tabular}




\section{Table 11.1 Volume Summary by Waste Stream Number (con't)}

\begin{tabular}{|c|c|c|c|}
\hline $\begin{array}{c}\text { Waste } \\
\text { Stream No. }\end{array}$ & Waste Stream Name & Preferred Option (PO) & $\begin{array}{c}\text { Current } \\
\text { Cumulative } \\
\text { Inventory } \\
\text { through } \\
09 / 30 / 04 \\
\left(\mathbf{m}^{3}\right)\end{array}$ \\
\hline SR-W081 & $\begin{array}{l}\text { Reactive and Ignitable } \\
\text { Mixed Waste }\end{array}$ & $\begin{array}{l}\text { Deactivation followed by } \\
\text { thermal treatment or } \\
\text { stabilization at commercial } \\
\text { facility }\end{array}$ & 0.2 \\
\hline SR-W082 & $\begin{array}{l}\text { Radioactive Chemicals, } \\
\text { Metals, and Pesticides } \\
\text { (CMP) Soil }\end{array}$ & $\begin{array}{l}\text { Stabilization by } \\
\text { Commercial Vendor }\end{array}$ & 0.0 \\
\hline SR-W083 & $\begin{array}{l}\text { Mercury- and Chromium- } \\
\text { Contaminated Residues }\end{array}$ & $\begin{array}{l}\text { Waste Stream } \\
\text { Recharacterized }\end{array}$ & N/A \\
\hline SR-W084 & $\begin{array}{l}\text { Remediation Waste Soils } \\
\text { that Meet LDR }\end{array}$ & Meets Treatment Standard & 0.0 \\
\hline SR-W085 & $\begin{array}{l}\text { Rocky Flats Plutonium } \\
\text { Fluoride Residues }\end{array}$ & $\begin{array}{l}\text { Future Generation - } \\
\text { Treatment to be } \\
\text { Determined }\end{array}$ & 0.0 \\
\hline SR-W086 & $\begin{array}{l}\text { Characteristically } \\
\text { Hazardous Non- } \\
\text { Incinerable Solids }\end{array}$ & $\begin{array}{l}\text { Amalgamation at } \\
\text { Commercial Vendor }\end{array}$ & 8.4 \\
\hline $\begin{array}{l}\text { SR-W087 } \\
\text { (BT-W035, } \\
\text { part of BT- } \\
\text { W003) }\end{array}$ & $\begin{array}{l}\text { Bettis Atomic Power } \\
\text { Laboratory Contaminated } \\
\text { Oil }\end{array}$ & $\begin{array}{l}\text { Thermal Treatment, or } \\
\text { macroencapsulation or } \\
\text { stabilization at commercial } \\
\text { facility }\end{array}$ & 0.2 \\
\hline SR-W088 & $\begin{array}{l}\text { Sludges and Non-Debris } \\
\text { Solids* }\end{array}$ & $\begin{array}{l}\text { Stabilization by } \\
\text { commercial vendor }\end{array}$ & 16.9 \\
\hline SR-W089 & $\begin{array}{l}\text { TRU Mixed Waste From } \\
\text { Mound Site }\end{array}$ & $\begin{array}{l}\text { Characterization at SRS - } \\
\text { WIPP Disposal }\end{array}$ & 17.0 \\
\hline SR-W090 & $\begin{array}{l}\text { Elemental Mercury - High } \\
\text { Rad }\end{array}$ & $\begin{array}{l}\text { Treatment by aging F/B } \\
\text { mercury treatment* }\end{array}$ & 0.3 \\
\hline SR-W091* & $\begin{array}{l}\text { Contaminated Debris } \\
\text { (High Rad)* }\end{array}$ & $\begin{array}{l}\text { New Stream - Treatment } \\
\text { to be Determined* }\end{array}$ & 5.1 \\
\hline & & TOTALS & $143,148.6$ \\
\hline
\end{tabular}

\section{NOTES:}

The volumes may not represent actual volumes of mixed waste stored at SRS as of the date of the annual update. The volume of wastes stored in tanks or certain special containers marked with an asterisk $(*)$ is reported as net. Volume of wastes stored in other containers such as boxes or drums is reported as gross. Volumes have been updated from those volumes reported in the 2003 STP update due to waste treatment progress, waste generation, changes in volume of waste streams based on further characterization, reassignment of some stream volumes to different stream numbers, and inventory adjustments.

Waste streams with 0 volumes have been treated or otherwise managed in accordance with RCRA regulations, or are future waste streams. 
Waste streams with N/A in the volume columns have had their waste volume incorporated into other waste streams or, if they have been re-characterized, are no longer a part of the STP.

\section{Table 11.2 Volume Summary by Treatment Facility}

\begin{tabular}{|c|c|c|}
\hline $\begin{array}{c}\text { Waste } \\
\text { Stream No. }\end{array}$ & Waste Stream Name & $\begin{array}{c}\text { Current } \\
\text { Cumulative } \\
\text { Inventory } \\
\text { through } \\
09 / 30 / 04 \\
\left(\mathbf{m}^{3}\right)\end{array}$ \\
\hline \multicolumn{3}{|c|}{ Commercial Vendor Treatment } \\
\hline \multicolumn{3}{|c|}{ Treatment Standard - Thermal treatment } \\
\hline SR-W001 & Rad-Contaminated Solvents & 26.4 \\
\hline SR-W003 & Solvent Contaminated Debris (LLW) & 0.7 \\
\hline SR-W012 & Toxic Characteristic Solids & 12.5 \\
\hline SR-W018 & Listed Incinerable Solids & 0.0 \\
\hline SR-W028 & Mark 15 Filter Paper & 0.0 \\
\hline SR-W035 & Mixed Waste Oil-Sitewide & 27.8 \\
\hline SR-W042 & Paints and Thinners & 0.9 \\
\hline SR-W051 & $\begin{array}{l}\text { Spent Filter Cartridges and Carbon Filter } \\
\text { Media }\end{array}$ & 3.8 \\
\hline SR-W055 & $\begin{array}{l}\text { Job Control Waste Containing Solvent } \\
\text { Contaminated Wipes }\end{array}$ & 0.0 \\
\hline SR-W070 & Mixed Waste from Laboratory Samples & 39.8 \\
\hline SR-W071 & $\begin{array}{l}\text { Wastewater Suitable for Thermal Treatment } \\
\text { or Stabilization }\end{array}$ & 0.8 \\
\hline SR-W080 & $\begin{array}{l}\text { Charleston Naval Shipyard } \\
\text { (CN-W001, CN-W004) }\end{array}$ & 0.0 \\
\hline SR-W081 & Reactive/Ignitable Waste & 0.2 \\
\hline SR-W087 & $\begin{array}{l}\text { Bettis Atomic Power Laboratory } \\
\text { Contaminated Oil (BT-W035, part of BT- } \\
\text { W003) }\end{array}$ & 0.2 \\
\hline \multicolumn{3}{|c|}{ Ashcrete Stabilization } \\
\hline SR-W046 & Consolidated Incineration Facility Ash & N/A \\
\hline \multirow[t]{2}{*}{ SR-W047 } & $\begin{array}{l}\text { Consolidated Incineration Facility } \\
\text { Blowdown }\end{array}$ & N/A \\
\hline & Subtotal & 113.1 \\
\hline \multicolumn{3}{|c|}{ ffluent Treatment Project-Wastewater Treatment } \\
\hline SR-W041 & Aqueous Mercury and Lead & 0.0 \\
\hline \multirow[t]{2}{*}{ SR-W077 } & Aqueous Characteristic Wastewater & 5.5 \\
\hline & Subtotal & 5.5 \\
\hline
\end{tabular}




\section{Table 11.2 Volume Summary by Treatment Facility} (con't)

\begin{tabular}{|c|c|c|}
\hline $\begin{array}{c}\text { Waste } \\
\text { Stream No. }\end{array}$ & Waste Stream Name & $\begin{array}{c}\text { Current } \\
\text { Cumulative } \\
\text { Inventory } \\
\mathbf{9 / 3 0 / 0 4} \\
\left(\mathbf{m}^{3}\right)\end{array}$ \\
\hline \multicolumn{3}{|c|}{ Commercial Solidification Technology Development } \\
\hline SR-W045 & PUREX Waste & 88.0 \\
\hline \multicolumn{3}{|c|}{ Sample Receipts } \\
\hline SR-W008 & $\begin{array}{l}\text { Separations Area Sample Receipts from } \\
\text { SRNL }\end{array}$ & 36.7 \\
\hline
\end{tabular}




\section{Table 11.2 Volume Summary by Treatment Facility} (con't)

\begin{tabular}{|c|c|c|}
\hline $\begin{array}{c}\text { Waste } \\
\text { Stream No. }\end{array}$ & Waste Stream Name & $\begin{array}{c}\text { Current } \\
\text { Cumulative } \\
\text { Inventory } \\
\text { through } \\
09 / 30 / 04 \\
\left(\mathbf{m}^{3}\right)\end{array}$ \\
\hline \multicolumn{3}{|c|}{ High-Level Waste ITP Facility } \\
\hline SR-W020 & Salt Processing Filters & 0.0 \\
\hline \multicolumn{3}{|c|}{ D-Area Heavy Water Operations Facility } \\
\hline SR-W032A & Mercury-Contaminated Heavy Water & N/A \\
\hline \multirow[t]{2}{*}{ SR-W032B } & $\begin{array}{l}\text { Mercury-Contaminated Heavy Water } \\
\text { Residues }\end{array}$ & 0.0 \\
\hline & Subtotal & 0.0 \\
\hline \multicolumn{3}{|c|}{ Defense Waste Processing Facility } \\
\hline SR-W016 & 221-F Canyon High-Level Liquid Waste & 52,500 \\
\hline \multirow[t]{2}{*}{ SR-W017 } & 221-H Canyon High-Level Liquid Waste & 85,400 \\
\hline & Subtotal & 137,900 \\
\hline \multicolumn{3}{|c|}{ Meet Treatment Standards } \\
\hline SR-W024 & Tritium Gold Traps & 3.0 \\
\hline SR-W040 & Stabilized Sludge/LDR-Compliant Debris & 18.0 \\
\hline SR-W063 & Macroencapsulated Low-Level Waste & 0.0 \\
\hline \multirow[t]{2}{*}{ SR-W084 } & Remediation Waste Soils that Meet LDR & 0.0 \\
\hline & Subtotal & 21.0 \\
\hline \multicolumn{3}{|c|}{ Macroencapsulation as a 90-Day Generator } \\
\hline SR-W015 & Tritium Contaminated Equipment & 22.0 \\
\hline SR-W023 & Cadmium Safety/Control Rods & 3.5 \\
\hline \multirow[t]{2}{*}{ SR-W072 } & $\begin{array}{l}\text { Supernate or Sludge Contaminated Debris } \\
\text { from High-Level Waste (HLW) Operations }\end{array}$ & 0.0 \\
\hline & Subtotal & 25.5 \\
\hline \multicolumn{3}{|c|}{ M-Area Vendor Treatment Facility } \\
\hline SR-W005 & Mark 15 Filtercake & 0.0 \\
\hline SR-W029 & M-Area Sludge Treatability Samples & 0.0 \\
\hline SR-W031 & Uranium/Chromium Solution & 0.0 \\
\hline SR-W037 & M-Area Plating Line Sludge & 0.0 \\
\hline \multirow[t]{2}{*}{ SR-W038 } & Plating Line Sump Material & 0.0 \\
\hline & Subtotal & 0.0 \\
\hline
\end{tabular}




\section{Table 11.2 Volume Summary by Treatment Facility} (con't)

\begin{tabular}{|c|c|c|}
\hline $\begin{array}{c}\text { Waste } \\
\text { Stream No. }\end{array}$ & Waste Stream Name & $\begin{array}{c}\text { Current } \\
\text { Cumulative } \\
\text { Inventory } \\
\text { through } \\
09 / 30 / 04 \\
\left(\mathbf{m}^{3}\right)\end{array}$ \\
\hline \multicolumn{3}{|c|}{ SRS (Facility TBD)-Macroencapsulation } \\
\hline SR-W009 & Silver Coated Packing Material & 6.3 \\
\hline \multirow[t]{2}{*}{ SR-W060 } & Tritiated Water with Mercury & 0.4 \\
\hline & Subtotal & 6.7 \\
\hline \multicolumn{3}{|c|}{ Treatment by Aging Followed by Thermal treatment and/or Hg treatment } \\
\hline SR-W036 & Tritiated Oil with Mercury & 18.1 \\
\hline SR-W090 & Elemental Mercury (High Rad) & 0.3 \\
\hline \multicolumn{3}{|c|}{ Offsite Vendor Facility - Macroencapsulation } \\
\hline SR-W062 & Low-Level Contaminated Debris & 10.8 \\
\hline \multirow[t]{2}{*}{ SR-W069 } & $\begin{array}{l}\text { Low-Level Waste (LLW) Lead-to be } \\
\text { Macroencapsulated Onsite }\end{array}$ & 124.6 \\
\hline & Subtotal & 135.4 \\
\hline \multicolumn{3}{|c|}{ Offsite Vendor Stabilization } \\
\hline SR-W048 & Soils from Spill Remediation & 0.0 \\
\hline SR-W049 & Tank E-3-1 Clean Out Material & N/A \\
\hline SR-W073 & Cadmium-Containing Raschig Rings & 0.0 \\
\hline SR-W082 & $\begin{array}{l}\text { Radioactive Chemicals, Metals, and } \\
\text { Pesticides (CMP) Soil }\end{array}$ & 0.0 \\
\hline \multirow[t]{2}{*}{ SR-W088 } & Sludges and Non-Debris Solids & 16.9 \\
\hline & Subtotal & 16.9 \\
\hline \multicolumn{3}{|c|}{ Offsite Vendor Facility-Amalgamation } \\
\hline SR-W014 & Elemental (Liquid) Mercury - Sitewide & 0.6 \\
\hline \multirow[t]{2}{*}{ SR-W086 } & $\begin{array}{l}\text { Characteristically Hazardous Non- } \\
\text { Incinerable Solids }\end{array}$ & 8.4 \\
\hline & Subtotal & 9.0 \\
\hline
\end{tabular}

Table 11.2 Volume Summary by Treatment Facility (con't) 


\begin{tabular}{|c|c|c|}
\hline $\begin{array}{c}\text { Waste } \\
\text { Stream No. }\end{array}$ & Waste Stream Name & $\begin{array}{c}\text { Current } \\
\text { Cumulative } \\
\text { Inventory } \\
\text { through } \\
9 / 30 / 04 \\
\left(\mathrm{~m}^{3}\right)\end{array}$ \\
\hline \multicolumn{3}{|c|}{ Offsite DOE-Combust in the TSCA Incinerator at ETTP } \\
\hline SR-W079 & $\begin{array}{l}\text { Polychlorinated Biphenyl (PCB) Mixed } \\
\text { Waste }\end{array}$ & 0.0 \\
\hline \multicolumn{3}{|c|}{ Waste Streams to be Further Characterized } \\
\hline None & & \\
\hline \multicolumn{3}{|c|}{ Offsite Vendor Deactivation } \\
\hline SR-W034 & Calcium Metal & 0.0 \\
\hline
\end{tabular}

Table 11.2 Volume Summary by Treatment Facility (con't) 


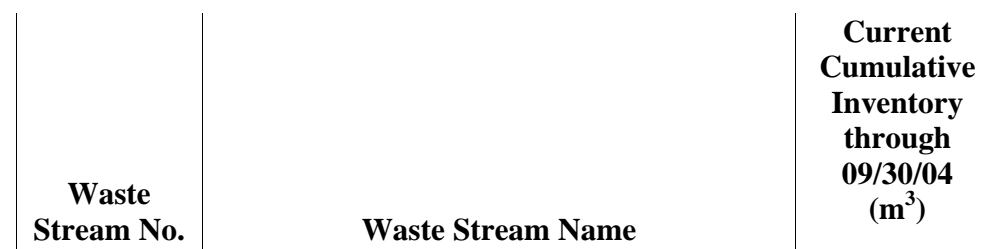

TRU Waste Streams Undergoing Characterization/Certification for
Shipment to WIPP
\begin{tabular}{|l|l|r|}
\hline SR-W006 & CH Mixed TRU/Liquids & 0.1 \\
\hline SR-W026 & CH Mixed TRU/Thirds & 175.6 \\
\hline SR-W027 & CH Mixed TRU/F-listed Solvents & 4574.4 \\
\hline SR-W053 & Rocky Flats Ash & 0.1 \\
\hline SR-W089 & TRU Waste From Mound Subtotal & 17.0 \\
\hline & & 4767.2 \\
\hline
\end{tabular}

\begin{tabular}{|c|c|c|}
\hline \multicolumn{3}{|c|}{$\begin{array}{l}\text { Lab Waste Treated as a 90-day Generator at SRNL followed by } \\
\text { Vitrification }\end{array}$} \\
\hline SR-W050 & $\begin{array}{l}\text { Waste to Support High-Level Waste (HLW) } \\
\text { Processing Demonstrations }\end{array}$ & 0.1 \\
\hline \multirow[t]{2}{*}{ SR-W058 } & $\begin{array}{l}\text { Mixed Sludge Waste with Mercury from } \\
\text { DWPF Treatability Studies }\end{array}$ & 0.0 \\
\hline & Subtotal & 0.1 \\
\hline
\end{tabular}

Scrap Metal Exclusion

\begin{tabular}{|l|l|l} 
SR-W011 & Cadmium Coated HEPA Filters & 0.0
\end{tabular}

\begin{tabular}{|c|c|c|}
\hline \multicolumn{3}{|c|}{ Future Generation - Treatment Plan to be Developed } \\
\hline SR-W085 & Rocky Flats Plutonium Fluoride Residues & 0.0 \\
\hline \multirow[t]{2}{*}{ SR-W091 } & Contaminated Debris - High Rad & 5.1 \\
\hline & Subtotal & 5.1 \\
\hline \multicolumn{3}{|c|}{ Waste Streams Consolidated } \\
\hline SR-W002 & Rad-Contaminated Chlorofluorocarbons & N/A \\
\hline SR-W004 & $\begin{array}{l}\text { M-Area Plating Line Sludge from Supernate } \\
\text { Treatment }\end{array}$ & N/A \\
\hline SR-W010 & Scintillation Solution & N/A \\
\hline SR-W019 & 244-H RBOF High Activity Liquid Waste & N/A \\
\hline SR-W025 & Solvent /TRU Job Control Waste $<100 \mathrm{nCi} / \mathrm{g}$ & N/A \\
\hline SR-W030 & Spent Methanol Solution & N/A \\
\hline SR-W033 & Thirds/TRU Job Control Waste $<100 \mathrm{nCi} / \mathrm{g}$ & N/A \\
\hline SR-W043 & Lab Waste with Tetraphenyl Borate & N/A \\
\hline SR-W044 & Tri-Butyl-Phosphate \& n-Paraffin-TRU & N/A \\
\hline SR-W054 & Enriched Uranium Contaminated with Lead & N/A \\
\hline SR-W059 & Tetrabutyl Titanate (TBT) & N/A \\
\hline SR-W061 & DWPF Mercury & N/A \\
\hline
\end{tabular}

Table 11.2 Volume Summary by Treatment Facility (con't) 


\begin{tabular}{|c|l|c|}
\multicolumn{1}{|c|}{$\begin{array}{c}\text { Waste } \\
\text { Stream No. }\end{array}$} & \multicolumn{1}{|c|}{$\begin{array}{c}\text { Current } \\
\text { Cumulative } \\
\text { Inventory } \\
\text { through } \\
\mathbf{0 9 / 3 0 / 0 4} \\
\left.\mathbf{( m}^{\mathbf{3}}\right)\end{array}$} \\
\hline SR-W068 & \multicolumn{1}{|c|}{ Elemental (Liquid) Mercury-Sitewide } & N/A \\
\hline Waste Streams & Re-characterized or Eliminated & N/A \\
\hline SR-W007 & SRNL Sample Material & N/A \\
\hline SR-W013 & $\begin{array}{l}\text { Low-Level Waste (LLW) Lead - To be } \\
\text { Decontaminated Onsite }\end{array}$ & N/A \\
\hline SR-W021 & Poisoned Catalyst Material & N/A \\
\hline SR-W045A & PUREX Aqueous Waste & N/A \\
\hline SR-W052 & Cadmium Contaminated Glovebox Section & N/A3,148.6 \\
\hline SR-W056 & $\begin{array}{l}\text { Job Control Waste with Enriched Uranium } \\
\text { and Solvent Contaminated Wipes }\end{array}$ & N/A \\
\hline SR-W057 & D-Tested Neutron Generators & TOTAL \\
\hline SR-W078 & $\begin{array}{l}\text { LDR Hazardous Waste Awaiting } \\
\text { Radiological Screening }\end{array}$ & $\begin{array}{c}\text { Mercury- and Chromium-Contaminated } \\
\text { Residues }\end{array}$ \\
\hline SR-W083 & \multicolumn{1}{|c|}{ N/A } \\
\hline
\end{tabular}

* Volume includes unstabilized ash and blowdown that does not meet the LDR standards.

** Mixed low-level waste conservatively managed as TRU (transuranic waste).

\section{NOTES:}

Volumes reflect inventories as of September 30, 2004. They may not represent actual volumes of mixed waste of SRS as of the date of the annual update.

Waste streams with 0 volumes have been treated or otherwise managed in accordance with RCRA regulations, or are future waste streams.

Waste streams with N/A in the volume columns have had their waste volume incorporated into other waste streams or, if they have been re-characterized, are no longer a part of the STP.

Totals do not include IDW streams 64, 65, 66, 67. 


\section{Table 11.3 Mixed Waste Treatment Residue Summary}

Residue from mixed waste treatment requiring RCRA Subtitle C disposal (as of 9/30/04).

\begin{tabular}{|l|l|l|l|}
\hline \multicolumn{1}{|c|}{ Waste Stream } & \multicolumn{1}{c|}{ Treatment } & \multicolumn{1}{c|}{ Residue Status } & \multicolumn{1}{c|}{ Comment } \\
\hline $\begin{array}{l}\text { SR-W015 } \\
\text { Mercury/Tritium } \\
\text { Contaminated Equipment }\end{array}$ & $\begin{array}{l}\text { Macroencapsulated in } \\
\text { a stainless steel } \\
\text { container }\end{array}$ & $\begin{array}{l}\text { Container stored at SRS in } \\
\text { Mixed Waste Storage } \\
\text { Buildings (645-2N and 643- } \\
\text { 29E) Total Volume }=22.0 \mathrm{~m}^{3}\end{array}$ & $\begin{array}{l}\text { Waste continues to be } \\
\text { generated. }\end{array}$ \\
\hline $\begin{array}{l}\text { SR-W023 } \\
\text { Cadmium Safety/Control } \\
\text { Rods }\end{array}$ & $\begin{array}{l}\text { Macroencapsulated in } \\
\text { a stainless steel } \\
\text { container. }\end{array}$ & $\begin{array}{l}\text { Container stored at SRS on } \\
\text { TRU Pad 12. Volume of waste } \\
=3.5 \mathrm{~m}^{3}\end{array}$ & $\begin{array}{l}\text { Total volume }=15.2 \mathrm{~m}^{3} \\
\text { Calculated from } \\
\text { container outside } \\
\text { dimension. }\end{array}$ \\
\hline $\begin{array}{l}\text { SR-W024 } \\
\text { Mercury/Tritium Gold }\end{array}$ & $\begin{array}{l}\text { Macroencapsulated in } \\
\text { a stainless steel } \\
\text { container. }\end{array}$ & $\begin{array}{l}\text { Containers stored at SRS } \\
\text { Mixed Waste Storage } \\
\text { Building (643-29E). } \\
\text { Total volume =3.0 } \mathrm{m}^{3} .\end{array}$ & \\
\hline $\begin{array}{l}\text { SR-W040 Listed } \\
\text { Stabilized Sludge/Listed } \\
\text { LDR Compliant Debris }\end{array}$ & $\begin{array}{l}\text { Stabilization at CIF } \\
\text { Ashcrete Unit or } \\
\text { Vitrification at M- } \\
\text { Area Vendor } \\
\text { Treatment Facility }\end{array}$ & $\begin{array}{l}\text { Containers of M area vitrified } \\
\text { glass were delisted and moved } \\
\text { to LLW disposal. }\end{array}$ & \\
\hline
\end{tabular}

\section{NOTES:}

The following characteristic waste streams have undergone treatment. However, treatment residues are not TCLP hazardous and do not require disposal in a RCRA Subtitle C facility: SR-W041, Aqueous Mercury and Lead; SR-W077, Aqueous Characteristic Wastewater; SR-W032B, Mercury-Contaminated Heavy Water Residues.

As of September 30, 2004, DWPF has produced or filled a total of 1710 stainless steel glass canisters, which have been placed in storage at SRS awaiting final disposition at Yucca Mountain.

As of November 5, 2001, all CIF stabilized ashcrete and blowdown had been shipped offsite for disposal.

The following material has been recycled in part or total. No residues have been generated requiring disposal as mixed waste by SRS: SR-W011, Cadmium Coated HEPA Filters; SR-W032, Mercury-Contaminated Heavy Water.

By previous agreement with SCDHEC, small (less than 55 gallons) quantities of mixed waste(s) stored in RCRA Satellite Accumulation Areas [R.61-79.262.34(c)] are not subject to R.61-79.268 and are not included in this plan, unless requested otherwise by SCDHEC. Also per agreement with SCDHEC, wastes stored in 90 day accumulation areas are not included in the waste inventory. 
THIS PAGE LEFT INTENTIONALLY BLANK. 


\section{Chapter 12. Acronyms and Definitions Glossary}

\section{Acronyms}

$$
-\mathbf{A}-
$$

ADGAS

AEA

Ag

ALARA

Am

AMALG

AOC

As

ASME

AVF

B/D

$\mathrm{Ba}$

BACT

BDAT

BIODG

BOD

$\mathrm{Br}$

BTU

C

$\mathrm{Ca}$

CAA

CAB

CBFO

CARBN

CB

CCMC

$\mathrm{Cd}$

$\mathrm{Ce}$

CEP

CEQ

CERCLA

Cf
Venting of compressed gases into an absorbing or reacting media

Atomic Energy Act

Silver

As Low As Reasonably Achievable

Americium

Amalgamation

Area of Contamination

Arsenic

American Society of Mechanical Engineers

Alpha Vitrification Facility

$$
-\mathbf{B}-
$$

Blowdown

Barium

Best Available Control Technology

Best Demonstrated Available Technology

Biodegradation

Biochemical Oxygen Demand

Bromine

British Thermal Unit

$$
-\mathbf{C}-
$$

Carbon

Calcium

Clean Air Act

Citizens Advisory Board

Carlsbad Field Office

Carbon Adsorption

Containment Building

Chemical Commodity Management Center

Cadmium

Cerium

Catalytic Extraction Processing

Council on Environmental Quality

Comprehensive Environmental Response, Compensation, and Liability Act

Consequence of Failure 
CFR

$\mathrm{CH}$

Chem

CHOXD

CHRED

$\mathrm{Ci}$

$\mathrm{CIF}$

$\mathrm{Cm}$

CMBST

CMP

CNS

Co

$\mathrm{CO}_{2}$

COBRA

Cont. Bldg.

$\mathrm{Cr}$

CRADA

Cs

CSSX

CSTP

CTF

CWA

${ }^{\circ} \mathrm{C}$

D\&D

DEACT

Decon

Dest

DETF

DF

Distill

DOD

DOE

DOE-AL

DOE-HQ

DOE-SR

DOT

DSTP

DWPF
Code of Federal Regulations

Contact Handled

Chemical

Chemical or Electrolytic Oxidation

Chemical Reduction

Curie

Consolidated Incineration Facility

Curium

Combustion

Chemicals, Metals, and Pesticides

Charleston Naval Shipyard

Cobalt

Carbon Dioxide

Computerized Radioactive Waste Burial Record Analysis

Containment Building

Chromium

Cooperative Research and Development Agreement

Cesium

Caustic Side Solvent Extraction

Conceptual Site Treatment Plan

Chemical Transfer Facility

Clean Water Act

Degrees Celsius

$$
-\mathbf{D}-
$$

Decontamination and Decommissioning

Deactivation

Decontamination

Destruction (Thermal Destruction)

Dilute Effluent Treatment Facility

Disposal Facility

Distillation

Department of Defense

U.S. Department of Energy

Department of Energy-Albuquerque Operations Office

U. S. Department of Energy-Headquarters

Department of Energy-Savannah River Operations Office

Department of Transportation

Draft Site Treatment Plan

Defense Waste Processing Facility 


$$
-\mathbf{E}-
$$

EA

EAV

ECA

ECM

EIS

EM

EMA

EPA

EPCRA

EPD

ER

ESP

ESS

ETF

ETTP

ETWAF

EU

$\mathrm{Eu}$

FBC

FFA

FFCA

FFCAct

FMWIR

FONSI

FP

FPR

FPTUR

FR

FSUBS

FY

FYP

g or gm

GAC

GAO

GOCO
Environmental Assessment

E-Area Vaults

Environmental Compliance Authority

Environmental Compliance Manual

Environmental Impact Statement

DOE Office of Environmental Management

WSRC Environmental Monitoring \& Analysis

U. S. Environmental Protection Agency

Emergency Planning and Community Right-to-Know Act

Environmental Protection Department

Environmental Restoration

Extended Sludge Processing

WSRC Environmental Services Section

Effluent Treatment Facility

East Tennessee Technology Park

Experimental Transuranic Waste Assay Facility

Enriched Uranium

Europium

$$
-\mathbf{F}-
$$

Fluidized Bed Combustion

Federal Facility Agreement

Federal Facility Compliance Agreement

Federal Facility Compliance Act

Final Mixed Waste Inventory Report

Finding of No Significant Impact

Filter Paper

Functional Performance Requirements

Filter Paper Take-Up Rolls

Federal Register

Fuel Substitution

Fiscal Year

Five Year Plan

$$
-\mathbf{G}-
$$

Gram

Granular Activated Carbon

General Accounting Office

Government Owned Contractor Operated 


\section{$-\mathbf{H}-$}

$\mathrm{H}$

$\mathrm{H}^{3}$

HATF

HBL

HEPA

HEU

$\mathrm{Hg}$

HL

HLLW

HLVIT

HLW

HSWA

HW

HW/MW

HW/MW DV

HW/MW-TB

HWCTR

HWSF

I

ICP

ID

IDMS

IDOA

IDW

IMERC

IMWIR

INCIN

INEEL

INTEC

ITP

IWPF

IWT

JCW
Hydrogen

Tritium

High Activity Transuranic Facility

Health Based Levels

High Efficiency Particulate Air

Highly Enriched Uranium

Mercury

High-Level

High-Level Liquid Waste

High-Level Vitrification

High-Level Radioactive Waste or High-Level Waste

Hazardous and Solid Waste Amendments

Hazardous Waste

Hazardous Waste/Mixed Waste

Hazardous Waste/Mixed Waste Disposal Vaults

Hazardous Waste/Mixed Waste Treatment Building

Heavy Water Components Test Reactor

Hazardous Waste Storage Facility

$$
-\mathbf{I}-
$$

Iodine

Ion Column Partitioning

Idaho

Integrated Defense Waste Processing Facility Melter System

In-Depth Options Analysis

Investigation or Investigative Derived Waste

Incineration of Wastes Containing Organics and Mercury

Interim Mixed Waste Inventory Report

Incineration

Idaho National Engineering and Environmental Laboratory

Idaho Nuclear Technology and Engineering Center

In-Tank Precipitation

Idaho Waste Processing Facility

Interim Waste Technology

$$
-\mathbf{J}-
$$

Job Control Wastes 


$$
-\mathbf{K}-
$$

K

$\mathrm{kg}$

1

LAER

LATF

LAW

LDR

LETF

LLNL

LLW

LW

$\mathrm{m}$

MACRO

mg

MGD

Mil

mil

MLLW

$\mathrm{mm}$

MOU

mrem

MSDS

MTRU

MWIP

MWIR

MWSB

MWST

$\mathrm{N}$

$\mathrm{Na}$

NASA

$\mathrm{Nb}$

NDA

NDE
Potassium

Kilogram

$$
-\mathbf{L}-
$$

Liter

Lowest Achievable Emission Rate

Low Activity Transuranic (TRU) Facility

Low Activity Waste

Land Disposal Restrictions

Liquid Effluent Treatment Facility

Lawrence Livermore National Laboratory

Low-Level Waste

Late Wash

$$
-\mathbf{M}-
$$

Meter

Macroencapsulation

Milligram

Million gallons/day

Million

Millimeter

Mixed Low-Level Waste

Millimeter

Memorandum of Understanding

One-thousandth of a rem (Millirem)

Material Safety Data Sheet

Mixed Transuranic Waste

Mixed Waste Integrated Program

Mixed Waste Inventory Report

Mixed Waste Storage Building

Mixed Waste Storage Tanks

$$
-\mathbf{N}-
$$

Nitrogen

Sodium

National Aeronautics and Space Administration

Niobium

Non-Destructive Analysis

Non-Destructive Evaluation 
NEPA National Environmental Policy Act

NESHAP

NEUTR

$\mathrm{NF}$

NGA

$\mathrm{Ni}$

NMD

NMP

NMV

NOI

$\mathrm{Np}$

NPDES

NPL

NPV

NR

NRC

NTPO

NWPA

NWW

O

O\&M

OGC

OR

ORR

OSHA

OTD

OWST

Ox

P

PA

PAC

$\mathrm{Pb}$

$\mathrm{Pc}$

PCB

PCC

PEIS

Pf

Pm
National Emissions Standards for Hazardous Air Pollutants

Neutralization

Naval Fuels

National Governors Association

Nickel

No-Migration Determination

No-Migration Petition

No-Migration Variance

Notice of Intent

Neptunium

National Pollutant Discharge Elimination System

National Priorities List

Net Present Value

Naval Reactors

Nuclear Regulatory Commission

National Transuranic Program Office

Nuclear Waste Policy Act

Non wastewater

$$
-\mathbf{O}-
$$

Oxygen

Operations and Maintenance

Office of General Counsel

Oak Ridge

Operational Readiness Review

Occupational Safety and Health Administration

Office of Technology Development

Organic Waste Storage Tank

Oxidation

$$
-\mathbf{P}-
$$

Phosphorus

Performance Assessment

Powdered Activated Carbon

Lead

Complexity Factor

Polychlorinated Biphenyl

Primary Combustion Chamber

Programmatic Environmental Impact Statement

Probability Factor

Maturity Factor 


$\begin{array}{ll}\text { Pm } & \text { Promethium } \\ \text { PO } & \text { Preferred Option } \\ \text { PPA } & \text { Pollution Prevention Act } \\ \text { PPE } & \text { Personal Protective Equipment } \\ \text { ppm } & \text { Parts Per Million } \\ \text { ppb } & \text { Parts Per Billion } \\ \text { ppt } & \text { Precipitate } \\ \text { Pr } & \text { Praseodymium } \\ \text { Pre-Op } & \text { Pre-Operational } \\ \text { Precip } & \text { Precipitation } \\ \text { PRECP } & \text { Precipitation } \\ \text { PSD } & \text { Prevention of Significant Deterioration } \\ \text { psig } & \text { Pounds per Square Inch Gauge } \\ \text { PSTP } & \text { Proposed Site Treatment Plan } \\ \text { Pu } & \text { Plutonium } \\ \text { Pu Sep } & \text { Plutonium Separation } \\ \text { PUREX } & \text { Plutonium Uranium Extraction } \\ \text { PVC } & \text { Polyvinyl Chloride } \\ \text { PWIT } & \text { Process Waste Interim Treatment } \\ \text { PWIT/SF } & \text { Process Waste Interim Treatment/Storage Facility } \\ \text { Pyrol } & \text { Pyrolysis }\end{array}$

\section{$-\mathbf{Q}-$}

QA

Quality Assurance

QC

Quality Control

$$
-\mathbf{R}-
$$

$\begin{array}{ll}\text { R\&D } & \text { Research and Development } \\ \text { R\&R } & \text { Roast/Retort } \\ \text { RA } & \text { Remedial Action } \\ \text { Rad } & \text { Radiation } \\ \text { RBOF } & \text { Receiving Basin for Offsite Fuel } \\ \text { RCA } & \text { Radiologically Controlled Area } \\ \text { RCRA } & \text { Resource Conservation and Recovery Act } \\ \text { React } & \text { Reaction } \\ \text { rem } & \text { Roentgen Equivalent Man } \\ \text { RF } & \text { Risk Factor } \\ \text { RFETS } & \text { Rocky Flats Environmental Technology Site } \\ \text { RFP } & \text { Request For Proposal } \\ \text { RH } & \text { Remote-Handled Waste } \\ \text { Rh } & \text { Rhodium } \\ \text { RL } & \text { Richland, Washington (Hanford) }\end{array}$


RLEAD

RMCP

RMERC

RMETL

RMMA

RO

ROD

RORGS

RTHRM

RTR

$\mathrm{Ru}$

S.S.

SAA

SAR

SARP

$\mathrm{Sb}$

$\mathrm{Sc}$

SCC

SCDHEC

SCHWMR

$\mathrm{Se}$

SED

SEIS

SFIA

SMPD

SNM

SR

$\mathrm{Sr}$

SR-WXXX

SRL

SRS

SRNL

Stab

STABL

STP

SWDF

SWMD

SWSP
Thermal Recovery of Lead

Residuals Management Contingency Plan

Retorting or Roasting

Recovery of metals or inorganics

Radioactive Materials Management Area

Reverse Osmosis

Record of Decision

Recovery of Organics

Thermal recovery of metals or inorganics

Real Time Radiography

Ruthenium

$$
-\mathrm{S}-
$$

Stainless Steel

Satellite Accumulation Area

Safety Analysis Report

Safety Analysis Report for Packaging

Antimony

Scandium

Secondary Combustion Chamber

South Carolina Department of Health and Environmental Control

South Carolina Hazardous Waste Management Regulation

Selenium

Special Equipment Development

Supplemental Environmental Impact Statement

Surplus Facilities Inventory Assessment

Sample Management Program Department

Special Nuclear Material

Savannah River

Strontium

Savannah River-Waste XXX

Savannah River Laboratory (old reference-currently known as Savannah River National Laboratory)

Savannah River Site

Savannah River National Laboratory (previously known as Savannah River Technology Center)

Stabilization

Stabilization

Site Treatment Plan

Solid Waste Disposal Facility

Solid Waste Management Department

Solid Waste Storage Pads 


$$
-\mathbf{T}-
$$

TAC Technical Advisory Committee

TB

TBD

TBT

TC

$\mathrm{Tc}$

TCLP

TDEC

TEC

Thermal Dest

TOC

TPB

TRU

TSCA

TSD

TSF

TSS

TTA

TWCCF

TWF

U

USAEC

USC

USC

USQ

UTS

UV

VE

VES

VOC

Vol

VTF

WAC

WAP
Treatment Building

To Be Determined

Tetrabutyl Titanate

Toxic Characteristic

Technetium

Toxicity Characteristic Leaching Procedure

Tennessee Department of Environmental Conservation

Total Estimated Cost

Thermal Destruction

Total Organic Carbon

Tetraphenyl borate

Transuranic

Toxic Substances Control Act

Treatment, Storage, and Disposal

Technology Success Factor

Total Suspended Solids

Thenoyl Trifluoroacetone

Transuranic Waste Certification/Characterization Facility

Transuranic Waste Facility

$$
-\mathbf{U}-
$$

Uranium

United States Atomic Energy Commission

University of South Carolina

United States Code

Unreviewed Safety Question

Universal Treatment Standards

Ultraviolet

$$
-\mathbf{V}-
$$

Visual Examination

Vinyl Ester Styrene

Volatile Organic Compounds

Volume

Vendor Treatment Facility

$$
-\mathbf{W}-
$$

Waste Acceptance Criteria

Waste Analysis Plan 
WBS

WEDF

WERF

WIPP

WITS

WMEIS

WMPEIS

WMin/PP

WSRC

Wt

WW

WWT

WWTF

$\mathrm{Zr}$
Work Breakdown Structure

Waste Engineering Development Facility

Waste Experimental Reduction Facility

Waste Isolation Pilot Plant

Waste Information Tracking System

Waste Management Environmental Impact Statement

Waste Management Programmatic Environmental Impact Statement

Waste Minimization/Pollution Prevention

Westinghouse Savannah River Company

Weight

Wastewater

Wastewater Treatment

Wastewater Treatment Facility

$$
-\mathbf{X}-
$$

$-\mathbf{Y}-$

Yttrium

$$
-\mathbf{Z}-
$$

Zirconium 


\section{Definitions}

The following definitions are provided to assist the reader with the specialized language in the STP. Effort has been made to assure that regulatory definitions listed in the STP are identical in wording with the appropriate definition in state and/or federal regulations. Where there are differences, regulatory definition wording takes precedence over that found in this definition section in the STP.

Amalgamation (AMLGM)-a process applicable to radioactive elemental mercury wastes. Mercury is converted into a solid alloy, which is more easily managed and less mobile than solutions containing radioactive mercury. Amalgamation provides a significant reduction in air emissions of mercury, and provides a change in mobility from liquid mercury to a paste-like solid, potentially reducing leachability. R.61-79.268.42 of the South Carolina Hazardous Waste Management Regulations (SCHWMR) defines amalgamation as amalgamation of liquid, elemental mercury contaminated with radioactive materials utilizing inorganic reagents such as copper, zinc, nickel, gold, and sulfur that result in a nonliquid semisolid amalgam and thereby reducing potential emission of elemental mercury vapors to the air.

Aqueous Liquids (as a waste matrix)-liquids/slurries with a total organic carbon (TOC) content less than $1 \%$. Slurries must be pumpable (e.g., suspended/settled solids can be up to approximately $35-40 \%$ ). Only liquids/slurries packaged/stored in bulk form (i.e., tank stored, drummed bulk free liquids) are included in this category. Liquids packaged in lab pack-type configuration are categorized as lab packs.

Back-logged waste-For the purpose of Section 3.1.1.1 of Volume I, back-logged waste is defined as incinerable waste which has been received into storage at RCRA permitted or RCRA interim status storage facilities as of 9/30/97. (Note: the 9/30/97 volume of backlogged incinerable mixed waste is $888 \mathrm{~m}^{3}$ of which $729 \mathrm{~m}^{3}$ is non-PUREX incinerable mixed waste, and $159 \mathrm{~m}^{3}$ is PUREX mixed waste. This volume differs from the 9/30/96 MWIR volumes as reported in the 1997 STP Annual Update because of receipt of additional mixed wastes into storage, shredding, and other volume adjustments determined during repackaging and further characterization efforts.)

Best Demonstrated Available Technology (BDAT)-to determine BDAT, the EPA examines all available performance data on technologies that are identified as demonstrating (using statistical techniques) whether one or more of the technologies performs significantly better than the others. The technology that performs "best" on a particular waste or waste treatability group is then evaluated to determine whether it is "available." To be available, the technology must be commercially available to any generator and provide "substantial" treatment of the waste, as determined through evaluation of accuracy-adjusted data. In determining whether treatment is substantial, EPA may consider data on the performance of a waste similar to the waste in question, provided that the similar waste is at least as difficult to treat. If the best technology is found to be not available, then the next best technology is evaluated, and so on.

Biodegradation (BIODG)-the degradation of organics or non-metallic inorganics (i.e., inorganics that contain phosphorous, nitrogen, and sulfur) in units operated under either aerobic or anaerobic conditions such that a surrogate compound or indicator parameter has been substantially reduced in concentration in the residuals (e.g., total organic carbon can often be used as an indicator parameter for the biodegradation of many organic constituents that cannot be directly analyzed in wastewater residues). Biodegradation is a hazardous waste treatment process identified in R.61-79.268.42 SCHWMR.

Borosilicate Glass-a type of heat-resistant glass containing at least $5 \%$ boric oxide (by weight); used in glassware that resists heat. Borosilicate glass is a leading candidate for use in high-level waste immobilization and disposal.

Capacity (of a facility)-the annual process throughput, in $\mathrm{m}^{3} / \mathrm{yr}$ under normal operating conditions. "Normal operating conditions" are the shift schedule under which the facility normally operates (i.e., one 8-hour shift/day, 5 days a week; two shifts/day, 5 days a week; 24 hours a day, 7 days a week). Facility operating capacity can be limited or regulated under a regulatory permit or interim status. 
Carbon Adsorption (CARBN)-a treatment technology used to treat wastewaters containing dissolved organics at concentrations less than about $5 \%$ and, to a lesser extent, dissolved metal and other inorganic contaminants. The two most common carbon adsorption processes are the granular activated carbon (GAC), which is used in packed beds, and the powdered activated carbon (PAC), which is added loosely to wastewater. R.61-79.268.42 SCHWMR defines carbon adsorption as: Carbon adsorption (granulated or powdered) of nonmetallic inorganics, organometallics and /or organic constituents operated such that a surrogate compound or indicator parameters has not undergone breakthrough (e.g., Total Organic Carbon can often be used as an indicator parameter for the adsorption of many organic constituents that cannot be directly analyzed in wastewater residues). Breakthrough occurs when the carbon has become saturated with the constituent (or indicator parameter) and substantial change in adsorption rate associated with that constituent occurs.

Cemented Solids (as a waste matrix)-sludges or solids (e.g., particulates, etc.) that have been solidified/stabilized with cement or other solidifying agents but do not meet LDR treatment standards. These wastes may require preparation for treatment (e.g., crushing/grinding) prior to subsequent LDR treatment.

Characterization-the determination of waste contents and properties, whether by review of process knowledge, nondestructive evaluation/nondestructive analysis (NDE/NDA) or sampling and analysis.

Chemical Fixations-any waste treatment process that involves reactions between the waste and certain chemicals, and results in solids that encapsulate, immobilize, or otherwise trap hazardous components in the waste to minimize the leaching of such components and to render the waste nonhazardous and more suitable for disposal.

Chemical Oxidation (CHOXD)-chemical or electrolytic oxidation utilizing the following oxidation reagents (or waste reagents) or combinations of reagents: (1) hypochlorite (e.g., bleach); (2) chlorine; (3) chlorine dioxide; (4) ozone or UV (ultraviolet light) assisted ozone; (5) peroxides; (6) persulfates; (7) perchlorates; (8) permanganates; and/or (9) other oxidizing reagents of equivalent efficiency, performed in units operated such that a surrogate compound or indicator parameter is substantially reduced in concentration in the residuals (e.g., total organic carbon can often be used as an indicator parameter for the oxidation of many organic constituents that cannot be directly analyzed in wastewater residues). Chemical oxidation specifically includes what is commonly referred to as alkaline chlorination. Chemical oxidation is a hazardous waste treatment process identified in R.61-79.268.42 SCHWMR.

Chemical Reduction (CHRED)-chemical reduction utilizing the following reducing reagents (or waste reagents) or combination of reagents: (1) sulfur dioxide; (2) sodium, potassium, or alkali salts of sulfites, bisulfites, metabisulfates, and polyethylene glycols (e.g., total organic halogens can often be used as an indicator parameter for the reduction of many halogenated organic constituents that cannot be directly analyzed in wastewater residues). Chemical reduction is commonly used for the reduction of hexavalent chromium to the trivalent state. Chemical reduction is a hazardous waste treatment process identified in R61-79.268.42 SCHWMR.

Cleanup-(1) actions undertaken during a removal or remedial response to physically remove or treat a hazardous substance that poses a threat or potential threat to human health and welfare, the environment, and/or real and personal property. Sites are considered cleaned up when removal or remedial programs have no further expectation or intention of returning to the site and threats have been mitigated or do not require action; or (2) actions taken to deal with a release or threat of release of a hazardous substance that could affect humans and/or the environment. The term "cleanup" is sometimes used interchangeably with either remedial action, removal action, response action, or corrective action.

Closure-Operational Closure-actions taken upon completion of operations to prepare the disposal site or disposal unit for custodial care (e.g., addition of cover, grading, drainage, erosion control). Final Site Closure: Actions taken as part of a formal decommissioning or remedial action plan, the purpose of which is to achieve long-term stability of the disposal site and to eliminate to the extent practical the need for active maintenance so that only surveillance, monitoring, and minor custodial care are required. 
Compliance Agreements-legally binding agreements between regulators and regulated entities that set standards and schedules for compliance with environmental statutes, including Consent Order and Compliance Agreements, Federal Facility Agreements, and Federal Facility Compliance Agreements.

Combustion (CMBST)-High temperature organic destruction technologies, such as combustion, in incinerators, boilers, or industrial furnaces operated in accordance with the applicable requirements of R.61-79.264, Subpart 0, or R.61-79.265, Subpart O, or R.61-79.266, Subpart H, of SCHWMR and in other units operated in accordance with applicable technical operating requirements; and certain noncombustive technologies, such as the Catalytic Extraction Process.

Concentration Based Standard-a land disposal restricted hazardous waste treatment standard for which the standard developed for an extract of the waste or treatment residue, or the constituent concentration in the waste or treatment residue has been determined at a specific maximum concentration level. These standards were based on best demonstrated available technology (BDAT) and the waste or waste extract or treatment residue must not exceed these concentrations if the waste is to be land disposed.

Contact-Handled Waste (CH)-waste or waste containers whose external surface dose rate does not exceed 200 mrem per hour at the surface of the container.

Container-any portable device in which a material is stored, transported, treated, disposed of, or otherwise handled (SCHWMR R.61-79.260.10 Subpart B Definitions).

Containment Building-a hazardous waste management unit used to store or treat hazardous waste under the provisions of Subpart DD of R.61-79 parts 264 and 265 SCHWMR

Corrosive/Corrosivity-(1) a solid waste exhibits corrosivity if a representative sample of the waste has either of the following properties (1) it is aqueous and has a $\mathrm{pH}$ less than or equal to 2 or greater than or equal to 12.5 as determined by a pH meter using Method 904D, "Test Methods for Evaluating Solid Waste Physical/Chemical Methods; or (2) it is a liquid and corrodes steel (SAE 1020) at a rate greater than $6.35 \mathrm{~mm}$ $\left(0.250\right.$ inch) per year at a test temperature of $55^{\circ} \mathrm{C}\left(130^{\circ} \mathrm{F}\right)$ as determined by the test method specified in NACE (National Association of Corrosion Engineers) Standard TM-01-69 as standardized in "Test Methods for the Evaluation of Solid Waste, Physical/Chemical Methods", EPA publication SW-846.

Curie-a measurement of a level of radiation activity in relation to the number of disintegrations per unit of time. One curie equals $2.7 \times 10^{10}$ disintegrations per second. Activity measured in milli $\left(10^{-3}\right)$, micro $\left(10^{-6}\right)$, nano $\left(10^{-9}\right)$, or pico $\left(10^{-12}\right)$ curie units is often expressed.

Deactivation (DEACT)-the removal of the hazardous characteristics of a waste due to its ignitability, corrosivity, and/or reactivity. Deactivation is a hazardous waste treatment process identified in R.61-79.268.42 SCHWMR.

Debris-solid material exceeding a 60-mm particle size that is intended for disposal and that is (1) a manufactured object; or (2) plant or animal matter; or (3) natural geologic material. However, the following materials are not debris: (1) any material for which a specific treatment standard is provided in Subpart D, part 268; (2) process residuals such as smelter slag and residues from the treatment of waste, wastewater, sludges or air emission residues; and (3) intact containers of hazardous waste that are not ruptured and that retain at least $75 \%$ of their original volume. A mixture of debris that has not been treated to the standards provided by R.61-79.268.45 SCHWMR and other material is subject to regulation as debris if the mixture is comprised primarily of debris by volume based on visual inspection. [From R.61-79.268.2(g) SCHWMR]

Decommissioning-(1) actions taken to reduce the potential health and safety impacts of contaminated DOE facilities, including activities to stabilize, reduce, or remove radioactive materials or to demolish the facilities; (2) preparations taken for retirement of a nuclear facility from active service, accompanied by the execution of a program to reduce or stabilize radioactive contamination; or (3) the process of removing a facility or area from operation and decontaminating and/or disposing of it or placing it in a condition of standby with appropriate controls and safeguards. 
Decontamination-the removal of unwanted material (typically radioactive material) from facilities, soils, or equipment by washing, chemical action, mechanical cleaning, or other techniques.

Defense Waste-(1) radioactive waste from any activity performed in whole or in part in support of DOE atomic energy defense activities; excludes waste under purview of the Nuclear Regulatory Commission or generated by the commercial nuclear power industry; or (2) nuclear waste derived mostly from the manufacture of nuclear weapons, weapons-related research programs, the operations of naval reactors, and the decontamination of production facilities.

Delist-use of the petition process to have a waste excluded from RCRA hazardous waste lists in Subpart D of Part 261.

Delisting-according to 40 CFR 260.20 and .22, to be exempted from the RCRA hazardous waste "system," a listed hazardous waste, a mixture of a listed and solid waste, or a derived-from waste must be delisted. Characteristic hazardous wastes never need to be delisted, but can be treated to eliminate the characteristic. A contained-in waste also does not have to be delisted; it only has to "no longer contain" the hazardous waste.

Department of Energy Waste-radioactive waste generated by activities of the DOE (or its predecessors), waste for which DOE is responsible under law or contract or other waste for which the DOE is responsible.

Derived-From Rule-This rule states that any solid waste derived from the treatment, storage, or disposal of a listed RCRA hazardous waste is itself a listed hazardous waste (regardless of the concentration of hazardous constituents) unless delisted per RCRA 40 CFR 260.22. For example, ash and scrubber water from the thermal treatment of a listed waste are hazardous wastes on the basis of the derived-from rule. Solid wastes derived from a characteristic hazardous waste are hazardous wastes only if they exhibit a hazardous characteristic.

Difficult-to-Treat Waste-Waste that does not meet the acceptance criteria of an offsite commercial vendor and cannot be readily handled by the commercial vendor under special procedures.

Disposal-the discharge, deposit, injection, dumping, spilling, leaking, or placing of any solid waste or hazardous waste into or on any land or water so that such solid waste or hazardous waste or any constituent thereof may enter the environment or be emitted into the air or discharged into any waters, including groundwaters (per SCHWMR R.61-79.260.10).

Disposal Facility-a facility or part of a facility at which hazardous waste is intentionally placed into or on the land or water, and at which waste will remain after closure. The term disposal facility does not include a corrective action management unit into which remediation wastes are placed (per SCHWMR R.61-79.260.10)

Effluent-(1) airborne and liquid wastes discharged from a site or facility following such engineering waste treatment and all effluent controls, including onsite retention and decay, as may be provided. This term does not include solid wastes, wastes for shipment offsite, wastes that are contained (e.g., underground nuclear test debris) or stored (e.g., in tanks) or wastes that are to remain onsite through treatment or disposal; or (2) wastewater (treated or untreated) that flows out of a treatment plant, sewer, or industrial outfall. Effluent may refer to wastes discharged into surface waters.

Elemental Lead (Activated and Non-Activated) (as a waste matrix)-both surface contaminated and activated elemental lead. Activated lead includes lead from accelerators or other neutron sources that may result in irradiation. Surface contaminated lead materials include bricks, counterweights, shipping casks, and other shielding materials.

Environmental Impact Statement (EIS)-(1) a document prepared in accordance with the requirements of $\S 102(2)(C)$ of National Environmental Policy Act (NEPA); or (2) a tool for decision making. It describes the positive and negative effects of the undertaking and lists alternative actions. The draft document (DEIS) is prepared by the DOE, or under DOE guidance, and attempts to identify and analyze the environmental impacts of a proposed action and feasible alternatives, and is circulated for public comment prior to preparation of the final environmental impact statement. 
Environmental Restoration (ER)-measures taken to clean up and stabilize or restore a site to regulatory acceptable conditions when the site has been contaminated with hazardous substances during past production or disposal activities.

Environmental Restoration Waste-waste generated by environmental restoration program activities.

Facility-all contiguous land, buildings, structures; other appurtenances, and improvements on the land used for treating, storing, or disposing of hazardous waste. A facility may consist of several treatment, storage, or disposal operational units (e.g., one or more or landfills, surface impoundments, or combinations of them per SCHWMR R.61-79.260.10).

Federal Facility Agreement (FFA)-Developed in response to requirements in Section 120 of the Comprehensive Environmental Response, Compensation, and Liability Act (CERCLA), the FFA is an interagency agreement between the Department of Energy-Savannah River Operations, the Environmental Protection Agency-Region IV, and the South Carolina Department of Health and Environmental Control to establish an expeditious schedule of remedial actions at contaminated sites placed on the National Priorities List. The FFA became effective on August 16, 1993.

Federal Facility Compliance Act of 1992 (FFCAct)-The FFCAct was passed by Congress and made effective on October 6, 1992. The FFCAct requires that except as provided below, after the date that is three years after the date of enactment of this Act, the waiver of sovereign immunity contained in Section 6001(a) of the Solid Waste Disposal Act with respect to civil, criminal, and administrative penalties and fines shall apply to departments, agencies, and instrumentalities of the executive branch of the federal government for violation of Section 3004(j) of the Solid Waste Disposal Act involving storage of mixed waste. With respect to the Department of Energy, the waiver of sovereign immunity referred to above shall not apply so long as the Department of Energy is in compliance with both (i) a plan that has been submitted and approved pursuant to Section 3021(b) of the Solid Waste Disposal Act and which is in effect; and (ii) an order requiring compliance with such plan which has been issued pursuant to such Section 3021(b) and which is in effect.

Federal Facility Compliance Agreement (FFCA)-an agreement between the DOE, a host state and/or EPA with respect to how and when some waste-related activity will be conducted to achieve compliance with applicable regulations in a timely manner. This agreement is a major driver or constraint on activities that sites must undertake for waste operations.

Filtration-removal/separation of particles from a mixture of fluid and particles by a medium that permits the flow of the fluid but retains the particles.

Free Liquid-means liquids which readily separate from the solid portion of a waste under ambient temperature and pressure (per SCHWMR R.61-79.260.10).

Fuel Substitution (FSUBS)-This treatment description has been replaced by the term Combustion (CMBST) per the LDR Phase III Rule.

Generator-any person, by site, whose act or process produces hazardous waste identified or listed in South Carolina Hazardous Waste Management Regulation R.61-79.261 or whose act first causes a hazardous waste to become subject to regulation per SCHWMR R.61-79.260.10.

Glovebox-(1) a sealed volume penetrated by leaded-rubber gloves that allows safe manipulation of some alpha-emitting particles; or (2) a windowed, low-leaking enclosure equipped with one or more pairs of flexible gloves to allow outside personnel to handle radioactive material within the enclosure.

Groundwater -means water below the land surface in a zone of saturation (per SCHWMR R.61-79.260.10).

Groundwater Contamination-the pollution of the underground sources of liquid water by potentially hazardous or toxic materials that move downward through the unsaturated profile to the zone of saturation or from improperly constructed or operated wells. 
Groundwater Remediation-treatment of groundwater to remove pollutants.

Hazardous Debris - means debris that contains a hazardous waste listed per Subpart D of Part 261 of SCHWMR or that exhibits a characteristic of hazardous waste identified in Subpart C of Part 261 of SCHWMR.

Hazardous Waste (HW)-those wastes that are designated hazardous by EPA (or state) Regulations. Those wastes listed by EPA (or state) or meeting characteristics specified by EPA (or state) in their criteria pursuant to RCRA. See South Carolina Hazardous Waste Management Regulations (SCHWMR) R.61-79.261.3 for specific detailed information.

Heterogeneous Debris (as a waste matrix)-wastes with matrices meeting the definition of debris per the August 18, 1992, LDR debris rule making (57 FR 37194, August 18, 1992). This category includes debris that do not meet the criteria for categorization as either Organic Debris or Inorganic Debris. This category also includes mixtures of debris and solid process residues or soil, provided debris comprises more than $50 \%$ of the waste.

High-Level Radioactive Waste (HLW)-(1) the highly radioactive waste material that results from the reprocessing of spent nuclear fuel including liquid waste produced directly in reprocessing and any solid waste derived from the liquid that contains a combination of transuranic (TRU) waste and fission products in concentrations requiring permanent isolation; or (2)(a) irradiated reactor fuel, (b) liquid wastes resulting from the operation of the first cycle solvent extraction system, or equivalent, and the concentrated wastes from subsequent extraction cycles, or equivalent, in a facility for reprocessing irradiated reactor fuel, and (c) solids into which such liquid wastes have been converted; or (3) as defined by the Nuclear Waste Policy Act (NWPA), (a) the highly radioactive material resulting from the reprocessing of spent nuclear fuel, including the liquid waste produced directly in reprocessing and any solid material derived from such liquid waste that contains fission products in sufficient concentrations; and (b) other highly radioactive material that the Nuclear Regulatory Commission (NRC), consistent with existing law, determines by rule to require permanent isolation; or (4) waste generated in the fuel of a nuclear reactor, or waste found at nuclear reactors or nuclear fuel reprocessing plants. These wastes are a serious threat to anyone who comes near them without shielding.

High-Level Vitrification (HLVIT)-vitrification of high-level radioactive wastes in units which comply with all applicable radioactive protection requirements under control of the Nuclear Regulatory Commission; or a mixed waste treatment process identified in R.61-79.268.42 of SCHWMR.

Ignitability/Ignitable-a waste property describing RCRA characteristically hazardous waste with a flash point lower than $140^{\circ} \mathrm{F}$. More detail on this definition can be found by consulting the SCHWMR R.61-79.261.21.

Immobilization-treatment of waste debris through macroencapsulation, micro-encapsulation, or sealing to reduce surface exposure to potential leaching media; or to reduce the leachability of the hazardous constituents. Described in Treatment Standards for Debris R.61-79.268.45 of SCHWMR.

Incineration (INCIN)-This treatment description has been replaced by the term Combustion (CMBST) per the LDR Phase III Rule.

Incineration of Wastes Containing Organics and Mercury (IMERC)-thermal treatment of wastes containing organics and mercury in units operated in accordance with the technical operating requirements of R.61-79.264 Subpart O and 265 Subpart O SCHWMR. All wastewater and nonwastewater residues derived from this process must then comply with the corresponding treatment standards per waste code with consideration of any applicable subcategories (e.g., high or low mercury subcategories) (per R.61-79.268.42 SCHWMR).

Inorganic Debris (as waste matrix)-wastes with matrices meeting the definition of debris per the August 18, 1992, LDR debris rule making (57 FR 37194, August 18, 1992). More specifically, this category is defined for wastes that contain $>90 \%$ inorganic debris. Examples include the following; metal shapes (e.g., equipment, scrap), metal turnings, glass (e.g., light tubes, leaded glass, etc.), ceramic materials, concrete, rocks. To meet the debris definition, material must be incapable of passing through a $9.5-\mathrm{mm}$ standard sieve. 
Inorganic Sludges/Particulates (as a waste matrix)-solid process residues with a predominately inorganic matrix. Solid process residues do not fit the definition of debris. Typically, these solids are sludge or particulate materials. Waste in this category may also contain some debris materials, provided the amount of debris is less than 50\% (based on LDR debris rule). The solids in this category may be contaminated with or contain organics such that thermal treatment is required. However, the matrices are predominantly inorganic so that thermal treatment would result in a high residue. Examples in this category are the following: sludges, ashes, and blasting media; absorbed aqueous or organic liquids (or inorganic particulate absorbents); ion exchange resins; and paint chips/residues.

Ion Exchange-a process that separates a mixed waste into its radioactive and/or hazardous constituents if the radioactive and/or hazardous components are ionic. It will also concentrate the radioactive and/or hazardous ionic species into a small volume, leaving a nonradioactive aqueous phase. The principal mixed waste application of this process is to recover metallic radionuclides from wastewaters or acid leach liquors. Ion exchange usually occurs through utilization of a resin which replaces the radioactive or hazardous ionic component with a nonradioactive or nonhazardous ionic component.

Job Control Waste (JCW)-discarded materials such as laboratory coats, plastic shoe covers, protective gloves and other paper, cloth, plastic, and glass products used in operations and preventive maintenance activities.

Lab Packs with Metals and Lab Packs without Metals (as waste matrices)-wastes with one or more small containers of free liquids or solids surrounded by solid materials (virgin or waste materials) within a larger container. Examples include scintillation fluids that are packaged with vials or containers of waste analytical reagents, used or unused laboratory samples, etc. The difference between wastes in these categories is contaminants. Lab packed wastes contaminated with TC metals are "Lab packs with Metals." Lab packed wastes not contaminated with TC metals are categorized as "Lab packs without Metals."

Land Disposal-placement in or on the land except in a corrective action management unit including, but not limited to, placement in a landfill, surface impoundment, waste pile, injection well, land treatment facility, salt dome, salt bed formation, underground mine or cave, or placement in a concrete vault or bunker intended for disposal purposes (per SCHWMR R.61-79.268.2(c)).

Land Disposal Restrictions (LDR)-(1) provisions of the Hazardous and Solid Waste Amendments (HSWA) requiring treatment of hazardous wastes before disposal; or (2) a RCRA program that restricts land disposal of RCRA hazardous wastes and requires treatment to promulgated treatment standards.

Land Disposal Restrictions-Federal Facility Compliance Agreement (LDR-FFCA)-An agreement effective March 13, 1991, between the Environmental Protection Agency-Region IV (EPA-IV) and the Department of Energy-Savannah River Operations (DOE-SR), which allowed the Savannah River Site (SRS) to continue to generate and store prohibited mixed waste regulated under the land disposal restrictions (LDR) of the Resource Conservation and Recovery Act (RCRA) while developing treatment capacity. The LDR-FFCA established a number of compliance deadlines involving LDR mixed waste treatment activities at SRS. The LDR-FFCA was amended three times. The third amendment, called the Bridging Amendment, was effective June 20, 1994, and aligned the LDR-FFCA with requirements of the Federal Facility Compliance Act (FFCAct). The LDR-FFCA expired on September 29, 1995, with agreement by SCDHEC and SRS on the FFCAct Consent Order and approval of the STP.

Leachate-any liquid, including any suspended components in the liquid, that has percolated through or drained from hazardous waste (per SCHWMR R.61-79.260.10). Leaching may occur at landfills or spill sites and may result in hazardous substances entering soil, surface water, or groundwater.

Listed Waste-wastes listed as hazardous under R.61-79.261 Subpart D SCHWMR, which includes lists of nonspecific source wastes, specific source wastes, and commercial chemical products or manufacturing chemical intermediates. These materials are listed because they exhibit a characteristic of hazardous waste, meet the statutory definition of hazardous waste, or are acutely toxic, acutely hazardous, or otherwise toxic. 
Liquid Mercury (as a waste matrix)-any wastes containing bulk volumes of elemental liquid mercury. The category includes lab packs of strictly liquid mercury or other containers containing bulk mercury.

Low-Level Radioactive Waste (LLW)-(1) waste that contains radioactivity and is not classified as high-level waste, transuranic (TRU) waste, or spent nuclear fuel, or the tailings or wastes produced by the extraction or concentration of uranium or thorium from any ore processed primarily for its source material content. Test specimens of fissionable material irradiated for research and development only, and not for the production of power or plutonium, may be classified as low-level waste, provided the concentration of TRU is less than 100 nanoCuries/gram (nCi/g); or (2) radioactive waste not classified as high-level waste, TRU waste, spent nuclear fuel, or byproduct material.

Macroencapsulation (MACRO) (technology based standard)-application of surface coating materials such as polymeric organics (e.g., resins and plastics) or with a jacket of inert inorganic materials to substantially reduce surface exposure to potential leaching media. Macroencapsulation specifically does not include material that would be classified as a tank or container according to R.61-79.260.10 SCHWMR. Macroencapsulation is a hazardous waste treatment process identified in R.61-79.268.42 SCHWMR.

Macroencapsulation (MACRO) (alternative standard for debris)-identical definition to the one immediately above for the technology based standard except this definition excludes the last sentence referring to use of materials that could be classified as a tank or container. A hazardous debris treatment identified in 40 CFR 268.45 of SCHWMR.

Metals Recovery (RMETL)-recovery of metals or inorganics utilizing one or more of the following direct physical/removal technologies: (1) ion exchange; (2) resin or solid (i.e., zeolites) adsorption; (3) reverse osmosis; (4) chelation/solvent extraction; (5) freeze crystallization; (6) ultrafiltration and/or (7) simple precipitation (i.e., crystallization). Note: This does not preclude the use of other physical phase separation or concentration techniques such as decantation, filtration (including ultrafiltration), and centrifugation, when used in conjunction with the above listed recovery technologies. Metals recovery is a hazardous waste treatment process identified in R.61-79.268.42 SCHWMR.

Microencapsulation-stabilization of the debris with the following reagents (or waste reagents) such that the leachability of the hazardous contaminants is reduced; (1) Portland cement; or (2) lime/pozzolans (e.g., fly ash and cement kiln dust). Reagents (e.g., iron salts, silicates, and clay) may be added to enhance the set/cure time and/or compressive strength or to reduce the leachability of the hazardous constituents. Microencapsulation is a hazardous debris treatment identified in R.61-79.268.45 of SCHWMR.

Mixed Low-Level Waste (MLLW)-low-level waste that also includes hazardous materials as identified in R.61-79.261, Subparts C and D, SCHWMR.

Mixed TRU (MTRU) Waste-Transuranic (TRU) waste that also includes hazardous materials as identified in R.61-79.261, Subparts C and D, SCHWMR.

Mixed Waste-waste that contains both hazardous waste and source, special nuclear, or by-product material subject to the Atomic Energy Act of 1954 (42 USC 2011 et seq.) (from Sec 1004 of the Solid Waste Disposal Act-42 USC 6902).

Mixture Rule-under the mixture rule, when any solid waste and a listed hazardous waste is mixed, the entire mixture is a listed hazardous waste unless the listed waste is listed for exhibiting a characteristic of a hazardous waste. Mixtures of solid waste and listed hazardous waste that are listed solely for exhibiting a characteristic are not hazardous if the resulting mixture no longer exhibits any characteristic. Mixtures of solid wastes and characteristic hazardous wastes are hazardous only if the mixture exhibits a hazardous characteristic. [R.61-79.261.3(a)(2)].

Moratorium Waste-those Land Disposal Restrictions (LDR) wastes generated in areas with a potential for causing radioactive contamination or activation that are subject to the May 17, 1991, DOE moratorium on offsite shipment of hazardous waste to commercial treatment, storage, and disposal facilities. Also included in the 1991 moratorium are certain heterogeneous and homogeneous solids from which a representative sample for radiological screening purposes cannot be obtained until appropriate sampling protocols are established. 
Neutralization (NEUTR)-use of the following reagents (or waste reagents) or combinations of reagents: (1) acids, (2) bases, or (3) water (including wastewaters) resulting in a $\mathrm{pH}$ greater than 2 but less than 12.5 as measured in the aqueous residuals. Neutralization is a hazardous waste treatment process developed in R.6179.268.42 SCHWMR.

Nondefense-Related Waste- radioactive waste under the purview of the Nuclear Regulatory Commission or generated by the commercial nuclear power industry, and not derived from the manufacture of nuclear weapons, weapons related research programs, operations of naval reactors and the decontamination of production facilities.

Non-PUREX SRS mixed wastes - For the purpose of Section 3.1.1.1 of Volume I, Non-PUREX SRS mixed wastes are defined as those mixed wastes, exclusive of waste stream SR-W045, identified in Volume 1, Section 3.1.1.1 of the SRS Approved Site Treatment Plan, Revision 5, March 20, 1997.

Nonwastewater-waste that does not meet the criteria for wastewater found later in these definitions.

Normal Mixed Waste-waste that is similar to "standard commercial mixed waste."

Onsite-the same or geographically contiguous property which may be divided by a public or private right of way provided the entrance and exit between the properties is at a crossroads intersection and access is by crossing as opposed to going along the right-of-way. Noncontiguous properties owned by the same person, but connected by a right-of-way which he controls and to which the public does not have access is also considered onsite property (per SCHWMR R.61-79.260.10).

Onsite Facility-a hazardous waste treatment, storage, or disposal area that is located on the generating site.

Organic Debris (as a waste matrix)-wastes with matrices meeting the definition of debris per R.61-79.268.2 debris rule making (57 FR 37194, August 18, 1992). This category is defined for wastes that contain $>90 \%$ organic debris. Examples include rags (including "solvent rags") plastic/rubber, paper, wood, glovebox gloves (including lead-lined), and animal carcasses.

Organic Liquids (as a waste matrix)-liquids/slurries with a total organic carbon (TOC) content greater than or equal to $1 \%$. Slurries must be pumpable (e.g., suspended/settled solids can be up to approximately 35-40\%). Only liquids/slurries packaged/stored in bulk form (i.e., tank stored, drummed bulk free liquids) are included in this category. Liquids packaged in lab pack-type configuration are categorized as lab packs.

Organic Sludges/Particulates (as a waste matrix)-solid process residues with an organic matrix. Solid process residues are solids that do not fit the definition of debris. Typically, these solids are sludge or particulate materials. Waste in this category may also contain some debris materials, provided the amount of debris is less than $50 \%$ (based on LDR debris rule). As opposed to Inorganic Sludges/Particulates, wastes in this category would not leave a large residue when thermally treated. Example waste materials are organic sludges, (e.g., sewage sludges) activated carbon, organic resins, and absorbed liquids (organic particulate absorbents).

Permit-an authorization, license, or equivalent control document issued by South Carolina or EPA to implement the requirements of R.61-79.124 and part 270 or equivalent federal regulation. Permit includes RCRA permit by rule (270.60). Permit does not include RCRA interim status (270.70) or any permit which has not yet been the subject of federal agency action, such as a draft permit or a proposed permit.

pH-(1) used to describe the hydrogen ion activity of a system. The logarithm of the reciprocal of hydrogen ion concentration $\left(-\log _{10}[\mathrm{H}+]\right.$, where $[\mathrm{H}+]$ is hydrogen-ion concentration in moles per liter); or (2) a symbol for the degree of acidity or alkalinity.

Plutonium-Uranium Extraction (PUREX) Process-a solvent extraction process used in the reprocessing of uranium/plutonium-based nuclear fuels. 
Precipitation (PRECP)-chemical precipitation of metals and other inorganics to form insoluble precipitates of oxides, hydroxides, carbonates, sulfides, sulfates, chlorides, fluorides, or phosphates. The following reagents (or waste reagents) are typically used alone or in combination: (1) lime (i.e., containing oxides and/or hydroxides of calcium and/or magnesium); (2) caustic (i.e., sodium and/or potassium hydroxides); (3) soda ash (i.e., sodium carbonate); (4) sodium sulfide; (5) ferric sulfate or ferric chloride; (6) alum; or (7) sodium sulfate. Additional flocculating, coagulating, or similar reagents/processes that enhance sludge dewatering characteristics are not precluded from use. Precipitation is a hazardous waste treatment process developed in R.61-79.268.42 SCHWMR.

Preparation for Treatment Processes-processes (e.g., shredding, grinding, physical separation, etc.) that make the waste amenable to the treatment process that ultimately destroys, removes, or immobilizes the hazardous contaminants or characteristics.

Processing - For the purpose of Section 3.1.1.1 of Volume I, processing is defined as the treatment of incinerable mixed wastes to meet the LDR standards in effect as of 9/30/97.

Radiation-(1) ionizing radiation that includes any or all of the following; gamma rays and $\mathrm{x}$-rays, alpha and beta particles, high-speed electrons, neutrons, high-speed protons, and other atomic particles. This definition does not include nonionizing radiations such as sound, microwave, radiowave or visible, infrared, or ultraviolet light; or (2) refers to the process of emitting energy in the form of rays or particles that are thrown off by disintegrating atoms. The rays or particles emitted may consist of alpha, beta, or gamma radiation.

Radioactive Materials Management Area (RMMA)-an area in which the potential exists for contamination due to the presence of unencapsulated or unconfined radioactive material or an area that is exposed to beams or other sources of particles (neutron, protons, etc.) capable of causing activation. Any of the following areas constitute an RMMA; (1) radiological buffer areas (except those established for a radiation field only) and all areas they encompass; (2) radioactive management areas; (3) soil contamination areas and the surrounding area that is greater than twice the background level of radiation; (4) underground radioactive material areas that have undergone operations to expose radionuclides (e.g., excavation); or (5) the area inside the OSHA physical control (e.g., fence) that was established for an environmental restoration activity where radioactive material is present.

\section{Radioactive Mixed Waste-(See Mixed Waste)}

Radioactive Waste-(1) solid, liquid, or gaseous material that contains radionuclides regulated under the AEA of 1954, as amended, and of negligible economic value considering recovery costs; or (2) a solid, liquid, or gaseous material of negligible economic value that contains radionuclides in excess of threshold quantities. Radioactive waste does not include material contaminated by radionuclides from nuclear weapons testing.

Radioactivity-(1) the spontaneous nuclear decay of material with a corresponding release of energy in the form of particles and/or electromagnetic radiation; or (2) the property or characteristic of radioactive material to spontaneously "disintegrate" with the emission of energy in the form of radiation. The unit of radioactivity is the curie.

Radionuclide-(1) a species of atom having an unstable nucleus that is subject to spontaneous decay; or (2) any nuclide that emits radiation. A nuclide is a species of atom characterized by the constitution of its nucleus and hence by its number of protons, neutrons, and energy content.

Reactive Metals (as a waste matrix)-bulk reactive metals and equipment contaminated with reactive metals. Bulk reactive metals include sodium, alkali metal alloys, aluminum fines, uranium fines, zirconium fines, and other pyrophoric materials. Contaminated equipment includes piping, pumps, and other materials with a residue or reactive metals that cannot be separated from the equipment medium.

Reactivity-a solid waste exhibits the characteristic of reactivity if a representative sample of the waste has any of the following properties: (1) It is normally unstable and readily undergoes violent change without detonating. (2) It reacts violently with water. (3) It forms potentially explosive mixtures with water. (4) When mixed with water, it generates toxic gases, vapors, or fumes in a quantity sufficient to present a danger to 
human health and the environment. (5) It is a cyanide or sulfide bearing waste which when exposed to $\mathrm{pH}$ conditions between 2 and 12.5, and can generate toxic gases vapors or fumes in a quantity sufficient to present a danger to human health or the environment. (6) It is capable of detonation or explosive reaction if it is subjected to a strong initiating source or if heated under confinement. (7) It is readily capable of detonation or explosive decomposition or reaction at standard temperature and pressure. (8) It is a forbidden explosive as defined in 49 CFR 173.51, or a Class A explosive as defined in 49 CFR 173.53 or a Class B explosive as defined in 49 CFR 173.88. This definition comes from R.61-79.261.23 SCHWMR.

Recovery of Organics (RORGS)-recovery of organics utilizing one or more of the following technologies, (1) distillation, (2) thin film evaporation, (3) steam stripping, (4) carbon adsorption, (5) critical fluid extraction, (6) liquid-liquid extraction, (7) precipitation/ crystallization (including freeze crystallization), or (8) chemical phase separation techniques (i.e., addition of acids, bases, demulsifiers, or similar chemicals). Note: This does not preclude the use of other physical phase separation techniques such as a decantation, filtration (including ultrafiltration), and centrifugation when used in conjunction with the above listed recovery technologies. Recovery of organics is a hazardous waste treatment process developed in R.61-79.268.42 SCHWMR.

rem- Roentgen equivalent man-a measure of radiation equal to the dose in rad (radiation absorbed dose) or Roentgens multiplied by a quality factor measuring the effectiveness of the absorbed dose: mrem equals a millirem or one-thousandth of a rem.

Remedial Action (RA)-(1) activities conducted at DOE facilities to reduce potential risks to people and/or harm to the environment from radioactive and/or hazardous substance contamination; or (2) those actions consistent with permanent remedy taken instead of, or in addition to, removal action in the event of a release or threatened release of a hazardous substance into the environment to prevent or minimize the release of hazardous substances so that they do not migrate to cause substantial danger to present or future public health or welfare or the environment. The term includes, but is not limited to, such actions at the location of the release as storage, confinement, perimeter protection, clay cover, neutralization, cleanup of released hazardous substances or contaminated materials, recycling or reuse, diversion, destruction, segregation of reactive wastes, dredging, or excavations, repair or replacement of leaking containers, collection of leachate and runoff, onsite treatment or combustion, provision of alternative water supplies, and any monitoring reasonably required to ensure that such actions protect the public health and welfare and the environment. The term includes the costs of permanent relocation of residents and businesses and community facilities where the president determines that, alone or in combination with other measures, such relocation is more cost-effective than, and environmentally preferable to, the transportation, storage, treatment, destruction, or secured disposition offsite of such hazardous substances, or may otherwise be necessary to protect the public health or welfare. The term does not include offsite transport of hazardous substances or contaminated materials unless the president determines that such actions are more cost-effective than other remedial actions; will create new capacity to manage in compliance with Subtitle C of the SWDA, hazardous substances in addition to those located at the affected facility; or are necessary to protect public health or welfare or the environment from a present or potential risk that may be created by further exposure to the continued presence of such substances or materials [as defined by $\S 101(24)$ of CERCLA].

Remote-Handled Waste (RH)-packaged waste with an external surface dose rate that exceeds 200 mrem per hour.

Remote Handling-the handling of wastes from a distance so as to protect human operators from unnecessary exposure.

Resource Conservation and Recovery Act (RCRA) Part A Permit Application-the first part of a Resource Conservation and Recovery Act permit application that identifies treatment, storage, and disposal units within a facility for which a permit is requested.

Resource Conservation and Recovery Act (RCRA) Part B Permit Application- the detailed second part of a RCRA permit application that describes waste to be managed, waste quantities, and facilities.

Retorting or Roasting (RMERC)-retorting or roasting in a thermal processing unit capable of volatilizing mercury and subsequently condensing the volatilized mercury for recovery. The retorting or roasting unit (or 
facility) must be subject to one or more of the following: (a) a National Emissions Standard for Hazardous Air Pollutants (NESHAP) for mercury; (b) a Best Available Control Technology (BACT) or a Lowest Achievable Emission Rate (LAER) standard for mercury imposed pursuant to a Prevention of Significant Deterioration (PSD) limit; or (c) a state permit that establishes emission limitations (within meaning of section 302 of the Clean Air Act) for mercury. All wastewater and nonwastewater residues derived from this process must then comply with the corresponding treatment standards per waste code with consideration of any applicable subcategories (e.g., high or low mercury subcategories). Retorting or roasting is a hazardous waste treatment process identified in R.61-79.268.42 SCHWMR.

Segregation-the separation of waste materials to facilitate handling, storage, treatment, transportation, and/or disposal.

Site-the land or water area where any facility or activity is physically located or conducted, including adjacent land used in connection with the facility or activity.

Site Characterization-the program of exploration and research, both in the laboratory and in the field, undertaken to establish the geologic conditions and the ranges of those parameters of a particular site. Site characterization includes borings, surface excavations, excavation of exploratory shafts, limited subsurface lateral excavations, and borings and geophysical testing.

Site Closure and Stabilization-those actions that are taken upon completion of operations that prepare the disposal site for custodial care and ensure that the disposal site will remain stable and will not need ongoing active maintenance.

Sludge-any solid, semi-solid, or liquid waste generated from a wastewater treatment plant, water supply treatment plant, or air pollution control facility exclusive of treated effluent from a wastewater treatment plant.

Soil (as a waste matrix)-soils contaminated with hazardous constituents and radioactivity that are stored in waste containers. Soil (as a waste matrix) includes soils contaminated with organics, inorganics, or both.

Soil With $<\mathbf{5 0 \%}$ Debris (as a waste matrix)-soils contaminated with hazardous constituents and radioactivity that are stored in waste containers, including soils contaminated with organics, inorganics, or both. This category may include debris, provided it is less than $50 \%$ of the waste.

Stabilization (STABL)-a broad class of treatment processes that immobilize hazardous constituents in a waste. For treatment of metals in mixed low-level wastes and for TRU wastes containing low-level radioactive components, stabilization technologies will reduce the leachability of the hazardous metal constituents (regardless of whether the metals are radioactive) in nonwastewater matrices. R.61-79.268.42 SCHWMR defines stabilization as reaction with the following reagents (or waste reagents) or combination of reagents: (1) Portland cement; or (2) lime/pozzolans (e.g., flyash and cement kiln dust). This does not preclude the addition of reagents (e.g., iron salts, silicates, and clays) designed to enhance the set/cure time and/or compressive strength, or to overall reduce the leachability of the metal or inorganic.

Standard Commercial Mixed Waste-waste that meets the acceptance criteria of the commercial vendor or is readily handled by the commercial vendor under special procedures.

Steam Stripping-a continuous process conducted in a unit that consists of a boiler, a stripping column, a condenser, and a collection tank. Steam stripping of organics from liquid wastes utilizes direct application of steam to the wastes operated such that liquid and vapor flow rates, as well as, temperature and pressure ranges, have been optimized, monitored, and maintained. These operating parameters are dependent upon the design parameters of the unit such as the number of separation stages and the internal column design. Steam stripping results in a condensed extract high in organics that must undergo thermal treatment, reuse as a fuel, or other recovery/reuse and an extracted wastewater that must undergo further treatment as specified in the standard. 
Storage-(1) temporary holding of waste pending treatment or disposal. Storage methods include containers, tanks, waste piles, surface impoundments, and containment buildings; (2) the containment of hazardous waste, either on a temporary basis or for a period of years, in such a manner as not to constitute disposal of such hazardous waste; or (3) retrievable retention of waste pending disposal. SCHWMR R.61-79.260.10 defines storage as the holding of hazardous waste for a temporary period, at the end of which the hazardous waste is treated, disposed of, or stored elsewhere.

Supercompaction-a volume-reduction method relying on mechanical compaction.

Technology Based Standard-a restricted waste for which a technology based standard is specified may be land disposed after it is treated using that specified technology or an equivalent treatment method approved by the Administrator of the EPA.

Thermal Recovery of Lead (RLEAD)-thermal recovery of lead in secondary lead smelters. A technology based treatment standard defined in SCHWMR R.61-79.268.42.

Thermal Treatment-the treatment of hazardous waste in a device that uses elevated temperatures as the primary means to change the chemical, physical, or biological character or composition of the hazardous waste. Examples of thermal treatment processes are thermal treatment, pyrolysis, calcination, wet air oxidation, and microwave discharge.

Toxicity Characteristic Leaching Procedure (TCLP)-a test designed to determine the mobility of both organic and inorganic analytes present in liquid, solid, or multi-phase wastes. If a solid waste analyzed using this method or approved equivalent demonstrates contaminant levels in excess of the listed concentrations found in the RCRA regulations, the waste is hazardous for the characteristic of toxicity.

Transuranic Waste (TRU)-Transuranic waste is radioactive waste containing more than 100 nanocuries (3700 becquerels) of alpha-emitting transuranic isotopes per gram of waste, with half-lives greater than 20 years, except for: (1) high-level radioactive waste; (2) waste that the Secretary of Energy has determined, with the concurrence of the Administrator of the Environmental Protection Agency, does not need the degree of isolation required by the 40 CFR Part 191 disposal regulations; or (3) waste that the Nuclear Regulatory Commission has approved for disposal on a case-by-case basis in accordance with 10 CFR Part 61. [Source: WIPP Land Withdrawal Act of 1992, as amended].

Treatability Group-based on the radioactive characteristics, hazardous components, and physical/chemical matrices as discussed above, DOE has grouped its wastes to reflect salient treatment considerations for each waste stream. These "treatability groups" are used to relate waste streams and waste quantities to treatment facilities and technology development needs.

Treatment-any method, technique, or process, including neutralization, designed to change the physical, chemical, or biological character or composition of any hazardous waste so as to neutralize, recover energy or material resources from the waste, or so as to render such waste nonhazardous, or less hazardous, safer to transport, store or dispose of, or amenable for recovery, amenable for storage, or reduced in volume per SCHWMR R.61-79.260.10.

Treatment Facility-the specific area of land, structures, and equipment dedicated to waste treatment and related activities.

Treatment, Storage, and Disposal (TSD) Facility-any building, structure, or installation where a mixed or hazardous waste has been treated, stored, or disposed.

Treatment System-the equipment and processes used for similar waste types at treatment facilities. A treatment system is the unit treatment operation or sequence of unit treatment operations carried out on all wastes that enter the system (e.g., a treatment system may consist of chemical reduction followed by precipitation or an incinerator and a vitrification unit for the ash). 
Underlying Hazardous Constituent-means any constituent listed in 40 CFR 268.48 Table UTS-Universal Treatment Standards, except zinc, which can reasonably expected to be present at the point of generation of the hazardous waste at a concentration above the constituent-specific UTS treatment standard.

Unit-discrete part of a facility used to treat, store, or dispose of hazardous or mixed waste.

Universal Treatment Standards-concentration levels for the constituents listed in 40 CFR 268.48-Table UTS Universal Treatment Standards which are required to be met for underlying hazardous constituents in waste treated for land disposal.

Variance-any mechanism or provision which allows modification to or waiver of the generally applicable requirements of R.61-79.124, R.61-79.270, R.61-79.260 through R.61-79.266 SCHWMR.

Vitrification-(1) a waste treatment process in which calcined or another decomposed form of waste is mixed with glass and fused into a solid mass. The resultant mass is expected to remain a stable and insoluble form for long time periods, and thus will be a leading candidate for the most benign waste form for disposal (Vitrification with borosilicate glass is the BDAT for HLW and certain mixed waste streams); (2) the conversion of high-level waste materials into a glassy or noncrystalline solid for subsequent disposal; or (3) the process of immobilizing waste that produces a glass-like solid that permanently captures the radioactive materials. Per SCHWMR R.61-79.268.42, vitrification of high level mixed radioactive wastes in units in compliance with all applicable radioactive protection requirements under control of the Nuclear Regulatory Commission.

Volatile Organic Compound (VOC)-(1) any reactive organic compound; or (2) an organic compound that evaporates (volatilizes) readily at room temperature.

Waste Acceptance Criteria (WAC)-the criteria used to determine if waste and waste packages are acceptable for treatment, storage, transportation, and disposal purposes.

Waste Characterization-activities to determine the extent and nature of the waste. (Note: Waste characterization may be based on process knowledge, nonintrusive nondestructive examination/nondestructive assay [NDE/NDA], or intrusive examination such as sampling and analysis.)

Wasteform-the physical form of the waste such as sludges, combustibles, metals, etc.

Waste Isolation Pilot Plant (WIPP)-(1) the project authorized under $\$ 213$ of the DOE National Security and Military Applications of Nuclear Energy Authorization Act of 1980 (Public Law 96-164; 93 Stat. 1259, 1265) to demonstrate the safe disposal of radioactive waste materials generated by atomic energy defense activities; or (2) a research and development facility, located near Carlsbad, New Mexico, to be used for demonstrating the safe disposal of TRU wastes from DOE activities.

Waste Management-the planning, coordination, and direction of those functions related to generation, handling, treatment, storage, transportation, and disposal of waste as well as associated surveillance and maintenance activities.

Waste Minimization-(1) an action that effectively avoids or reduces the generation of waste by source reduction, improving energy usage, or by recycling. This action is consistent with the general goal of minimizing present and future threats to human health, safety, and the environment; or (2) the reduction, to the extent feasible, of hazardous waste that is generated prior to treatment, storage, or disposal of the waste. Waste minimization includes any source reduction or recycling activity that results in either (a) reduction of total volume of hazardous waste, (b) reduction of toxicity of hazardous waste or (c) both.

Waste Segregation-the separation of waste materials before the package (or repackage) process to facilitate handling, storage, treatment, transportation, and/or disposal.

Waste Stream-a general term developed for the STP used to describe a volume of waste collected from various locations or generated from a specific process with a similar physical matrix and treatment requirement. 
Wastewaters-wastes that contain less than $1 \%$ by weight total organic carbon (TOC) and less than $1 \%$ by weight total suspended solids (TSS) with the following exception: F001, F002, F003, F004, F005 wastewaters are solvent-water mixtures that contain less than $1 \%$ by weight TOC or less than $1 \%$ by weight total F001, F002, F003, F004, F005 solvent constituents listed in R.61-79.268.40, Table Constituent Concentrations in Waste Extract. 
THIS PAGE INTENTIONALLY LEFT BLANK. 